\title{
WestVirginiaUniversity
}

THE RESEARCH REPOSITORY @ WVU

Graduate Theses, Dissertations, and Problem Reports

2006

\section{Neutral density profiles in argon helicon plasmas}

Amy M. Keesee

West Virginia University

Follow this and additional works at: https://researchrepository.wvu.edu/etd

\section{Recommended Citation}

Keesee, Amy M., "Neutral density profiles in argon helicon plasmas" (2006). Graduate Theses,

Dissertations, and Problem Reports. 2441.

https://researchrepository.wvu.edu/etd/2441

This Dissertation is protected by copyright and/or related rights. It has been brought to you by the The Research Repository @ WVU with permission from the rights-holder(s). You are free to use this Dissertation in any way that is permitted by the copyright and related rights legislation that applies to your use. For other uses you must obtain permission from the rights-holder(s) directly, unless additional rights are indicated by a Creative Commons license in the record and/ or on the work itself. This Dissertation has been accepted for inclusion in WVU Graduate Theses, Dissertations, and Problem Reports collection by an authorized administrator of The Research Repository @ WVU.

For more information, please contact researchrepository@mail.wvu.edu. 


\title{
Neutral Density Profiles in Argon Helicon Plasmas
}

\author{
Amy M. Keesee \\ Dissertation submitted to the College of Arts and Sciences \\ at West Virginia University \\ in partial fulfillment of the requirements \\ for the degree of \\ Doctor of Philosophy \\ in \\ Plasma Physics \\ Earl Scime, PhD., Chair \\ Manfred Boehm, PhD. \\ Fred L. King, PhD. \\ Mark. E. Koepke, PhD. \\ John E. Littleton, $\mathrm{PhD}$. \\ Department of Physics \\ Morgantown, West Virginia \\ 2006
}

Keywords: helicon source, laser-induced fluorescence, neutral density, collisionalradiative, Abel inversion

Copyright 2006 Amy M. Keesee 


\title{
Abstract
}

\section{Neutral Density Profiles in Argon Helicon Plasmas}

\begin{abstract}
Amy M. Keesee
A diode laser-based laser-induced fluorescence (LIF) diagnostic has been developed that can measure three species; argon neutrals, argon ions, and helium neutrals. This diagnostic has been combined with passive emission spectroscopy and a neutral argon collisional-radiative (CR) model to measure ground state radial density profiles of argon atoms in a helicon source. We have found the ground state neutral argon atoms to have a $60 \%$ on-axis depletion for a typical helicon mode case, yielding a $28 \%$ ionization fraction. The depletion decreases to $20 \%$ with a $9.8 \%$ ionization fraction for a second helicon mode case, indicating that slight changes in plasma parameters can lead to a significant difference in RF power coupling and gas ionization. In a series of experiments in a low density helicon source, measurements of argon ion flow through a double layer with the LIF diagnostic confirmed predictions of a Monte-Carlo particle-incell model of double layer formation in expanding helicon plasmas. Additionally, the LIF diagnostic has been used to measure argon neutral flow velocities, argon ion flow velocities, and argon neutral density and temperature evolution during a plasma pulse.
\end{abstract}




\section{Acknowledgements}

First, I want to thank my advisor, Earl Scime, for inviting me to join his research group even when I was "just going to be around for two years," and for teaching me all the lab-related stuff I missed by not being a physics major.

Enormous thanks to Annemie Bogaerts for providing me with her CR model code as well as help in understanding and using it. I would like to thank all those who contributed to the data used in this dissertation, including Christine Charles, Robert Boivin, Costel Biloiu, Robert Hardin, Chris Compton, and Albert Meige. Special thanks go to Phil Tucker, Doug Mathess, Carl Weber, and Tom Milan for sharing their skills and expertise. Thank you to Ronald Bell for suggesting the matrix inversion technique.

I would like to thank those with whom I've had meaningful scientific discussions about the contents of this dissertation, including Christine Charles, Rod Boswell, Greg Severn, and Sam Cohen. I would also like to thank those who have given me helpful general advice throughout my graduate student career, including Martina Bachlechner, Christine Charles, Ed Thomas, Jr., Stewart Zweben, and Mark Koepke. Thanks to my committee for spending the time on this work, and also to Art Weldon for being a substitute member for my oral examination.

Finally, I would like to thank my husband, Sean, for his love and support, and for giving me the "unique German last name that every physicist needs." Special thanks go to my parents for always encouraging me to pursue whatever dreams I have had. I would like to dedicate this work to the child that has been with me through most of the writing

process and the defense, that he may be fascinated by the world into which he will soon enter and enjoy a life discovering the world's wonders. 


\section{Table of Contents}

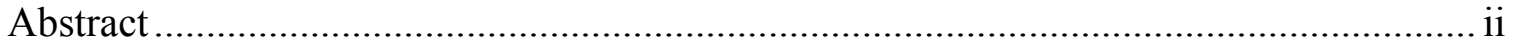

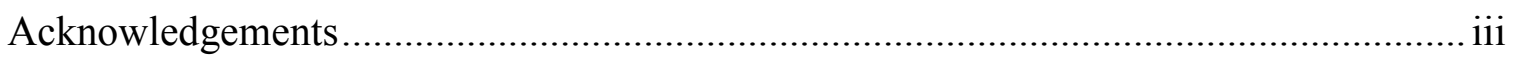

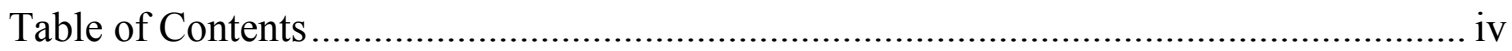

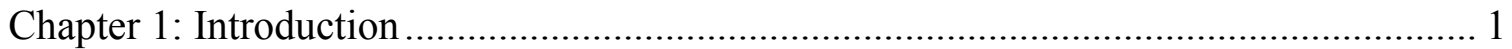

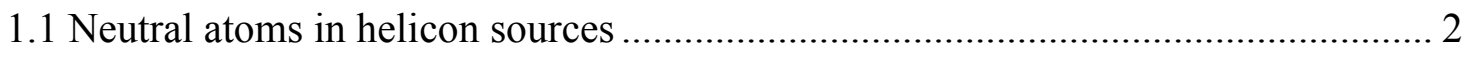

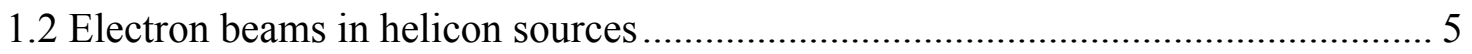

1.3 Flows and double layers in helicon sources............................................................ 7

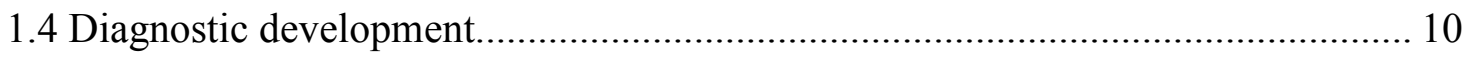

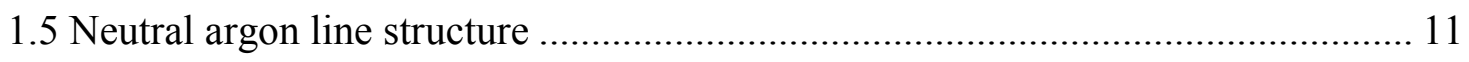

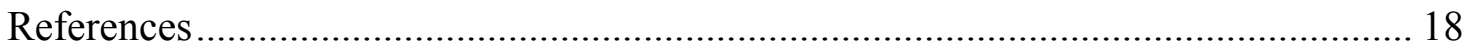

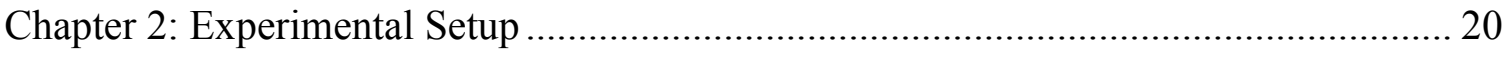

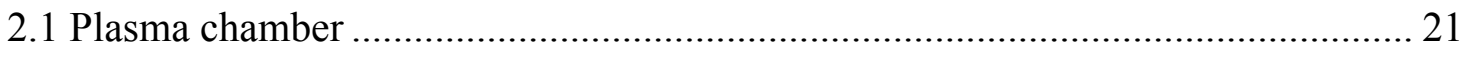

2.2 Vacuum system ............................................................................................ 23

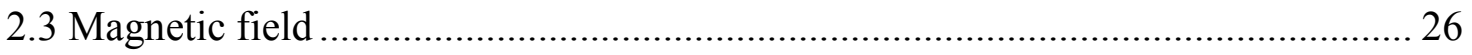

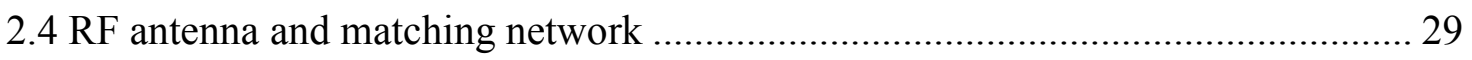

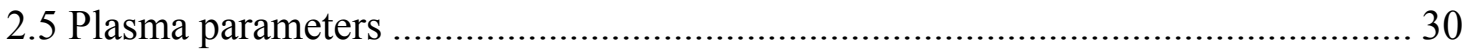

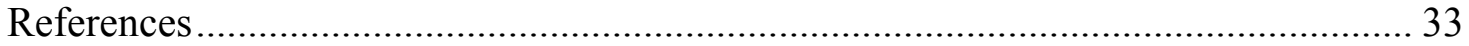

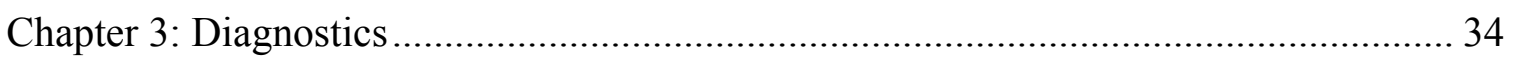

3.1 Laser-induced fluorescence ………………………........................................... 34

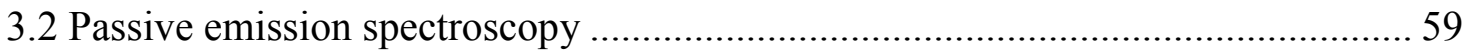

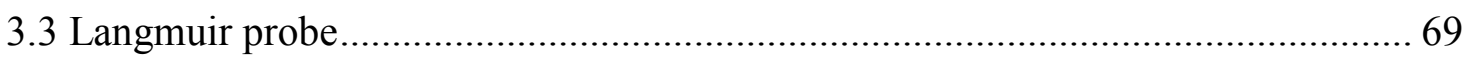

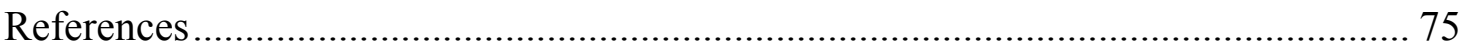

Chapter 4: ANU Experiments................................................................................. 76

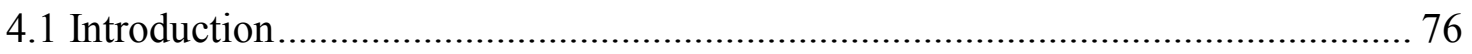

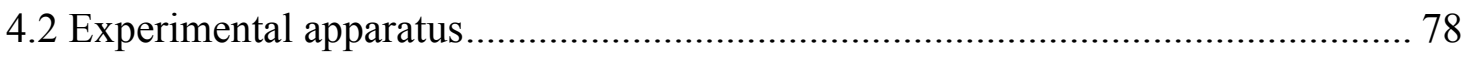

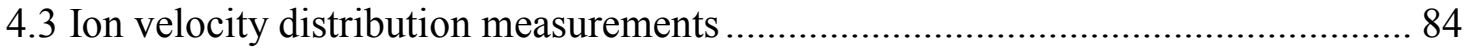

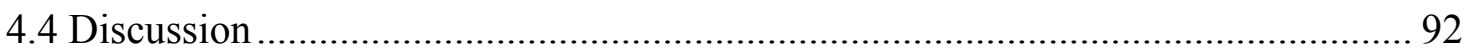

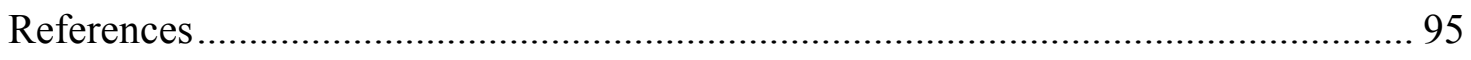

Chapter 5: Collisional-Radiative Modeling ................................................................... 96

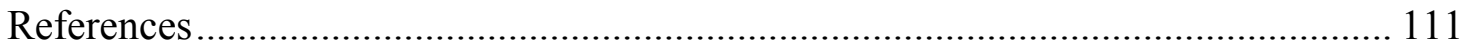


Chapter 6: Measurements of Radial Profiles, Temperatures and Flows in HELIX........ 112

6.1 Parameter Set A (6 mTorr Helicon mode) ...................................................... 113

6.2 Parameter Set B (Capacitive mode) ............................................................... 117

6.3 Parameter Set C (5 mTorr Helicon mode) ...................................................... 121

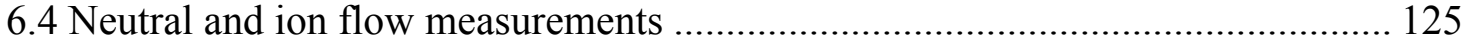

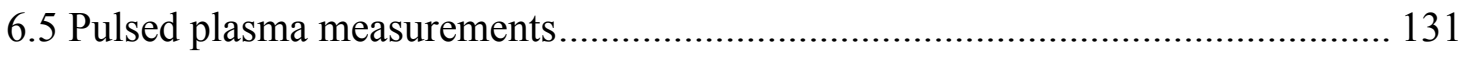

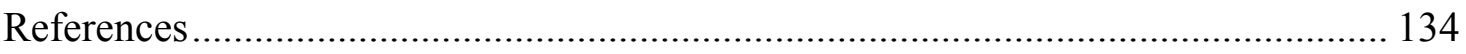

Chapter 7: Comparison of CR Model and Measurements ......................................... 135

7.1 Parameter Set A (6 mTorr Helicon mode) ..................................................... 136

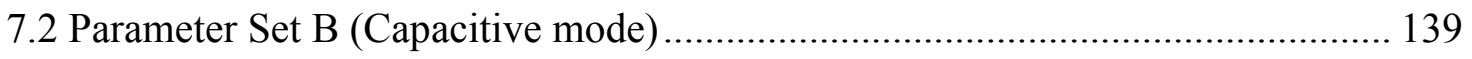

7.3 Parameter Set C (5 mTorr Helicon mode) .................................................... 155

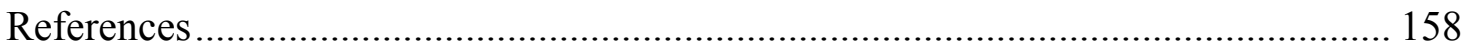

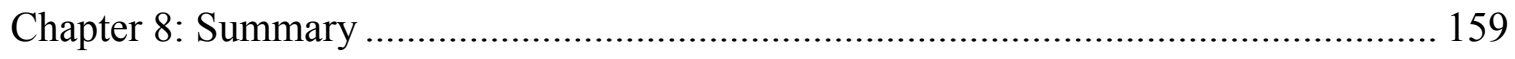

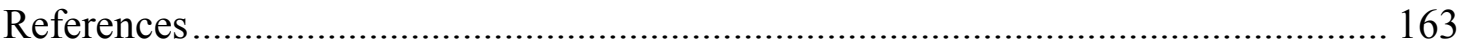

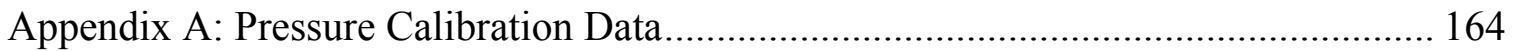

Appendix B: Iodine Cell Fluorescence Data ...................................................... 167

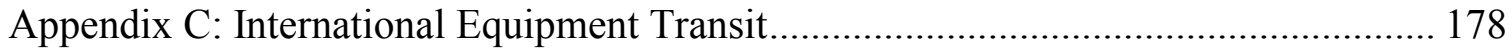

Appendix D: Collisional-Radiative Model Code........................................................ 183 


\section{Chapter 1: Introduction}

Helicon source plasmas are used for plasma processing, space simulation, space propulsion, and basic plasma physics studies. While plasma physics typically focuses on the dynamics of ions and electrons in the plasma, the neutral gas that is present in all plasmas can play a significant role in radiation losses, diffusion, momentum transport, and cooling. To understand the role of neutrals in helicon plasmas, accurate and precise measurements of neutral atom spatial distribution, temperature, density, and flow speed are required. In this work, it will be shown how laser-induced fluorescence (LIF) and passive emission spectroscopy provide non-perturbative measurements of these neutral atom properties in argon helicon source plasmas. Because both of these optical diagnostic methods probe an excited neutral state, a collisional-radiative (CR) model is also needed to relate the measured excited state properties to those of the neutral ground state. Thus, LIF measurements of excited state radial profiles and a CR model relating the excited state density to the ground state density yield a measurement of the ground state radial profile.

The atomic physics of the CR model, i.e., the rate of excitation and depopulation of the excited neutral states, is primarily controlled by the electron energy distribution function. Therefore, Langmuir probe measurements of electron temperature and density are an important input to the CR model and are also reported in this work for a series of helicon source configurations. Since different excited neutral states are investigated with LIF and passive emission spectroscopy, the possible existence of an electron beam (an 
important topic in helicon plasma source research) will also be considered in light of the experimental measurements and CR model results.

After reviewing some background information in the remainder of this chapter and then relevant experimental apparatus and diagnostic methods in Chapters 2 and 3, respectively, the review of experimental measurements in this work begins in Chapter 4 with measurements of the acceleration of argon ions through a spontaneously forming double layer (DL) in experiments at the Australian National University (ANU). The portable LIF diagnostic used to measure the neutral argon velocity distribution is also capable of measuring the velocity distribution of argon ions and neutral helium. In addition to providing confirmation of the existence of the DL in the ANU experiment, the initial argon ion LIF measurements demonstrated that satisfactory LIF signal to noise could be obtained at target species densities of as little as $10^{9} \mathrm{~cm}^{-3}$ and that the heated iodine cell used for absolute flow velocity reference could be reliably used for a wide range of experimental conditions. Details of the collisional-radiative model are discussed in Chapter 5, and the experimental measurements of neutral spatial distribution, neutral density, neutral temperature, neutral flow, and ion flow, are presented in Chapter 6 . Interpretation of the experimental measurements in light of the collisional-radiative model results is presented in Chapter 7, and a summary of the key results of this work are given in Chapter 8.

\subsection{Neutral atoms in helicon sources}

In a number of helicon source investigations, the absolute density and spatial distribution of neutral atoms in the source chamber remains an important and unanswered question. For example, plasma processing applications typically require plasma 
uniformity, though little is understood about the effects of neutral non-uniformity. ${ }^{1}$ Tynan measured the radial profile of neutral pressure in a helicon source used for plasma processing of microchips, finding a decrease of $\sim 20 \%$ to $40 \%$ toward the center of the plasma that was more pronounced for increased fill pressure. Because neutral pressure depends on the product of neutral density and temperature, such measurements cannot distinguish between density and temperature variations. His measurements were found to be in reasonable agreement with a simple 1-D diffusion model of neutral transport that assumed the neutral temperature was uniform and the radial variation of neutral density was small. In another investigation, measurements of Alfvén wave damping in helium helicon plasmas by Watts and Hanna appeared to be consistent with wave damping due to ion-neutral collisions. ${ }^{2}$ Using both a diffusion model similar to Tynan's and a collisionless transport model, they determined that at the center of the source, the helium plasma was approximately $3 \%$ ionized. In contrast to the Tynan and the Watts and Hanna measurements, Boswell postulated that fully ionized plasmas in the center of helicon discharges were required for pressure balance between the hot plasma in the center and the dense, cold neutrals at the plasma edge. ${ }^{3}$ Such hollow neutral density profiles are typical of high temperature laboratory plasmas, ${ }^{4}$ but the neutral density profile in the low temperature, high-density plasmas typical of helicon sources was never directly measured.

A few groups have investigated the phenomenon of neutral pumping ${ }^{5,6,7}$ in helicon sources. Neutral pumping occurs when neutral atoms are ionized in a linear plasma source and then are accelerated by the presheath electric field towards the chamber wall at the ion acoustic speed. Upon colliding with the wall the ions recombine, 
and the resulting neutrals are pumped away or slowly diffuse back toward the center at the thermal speed. The large difference in inward and outward speeds leads to a depletion of neutrals at the center of the plasma chamber. Gilland et al. measured axial neutral pressure profiles in a high power helicon source. ${ }^{5}$ In the absence of neutral temperature measurements, they had to assume that the neutrals were unheated and that the neutral density was directly proportional to the measured pressure. They found that their axial neutral pressure profile measurements were consistent with the predictions of a one dimensional diffusion model that assumed all neutrals crossing into the center of the discharge through a radial neutral-plasma boundary were ionized and then diffused to the end of the helicon source (with the diffusion being limited by charge exchange collisions). ${ }^{5}$ Although their model assumed a distinct boundary between cold, dense, neutral gas at the edge and a hot, highly ionized plasma in the center of the discharge, they did not report any radially resolved neutral density measurements and assumed a uniform radial neutral density at each axial location. It is important to note that the reported on-axis neutral and plasma densities indicated that the core plasma was only weakly ionized $(<15 \%)$.

In a different helicon source experiment, Degeling et al. hypothesized that neutral pumping was responsible for a relaxation oscillation between a high-density helicon wave mode and a lower-density, inductively coupled mode. By allowing neutral pumping effects to reduce the neutral density in the plasma source, they were able to reproduce the observed relaxation oscillation frequency with a pair of coupled, nonlinear, differential equations that described the neutral and plasma densities in the source chamber. ${ }^{6}$ In yet another helicon plasma source, Yoon et al. incorporated neutral pumping into a global 
model of plasma density and electron temperature. In the model, they assumed that neutrals were completely depleted from the center of the discharge during the "highcurrent phase" of the pulse. Their model yielded current densities that agreed with experimental measurements in their modeled helicon source. ${ }^{7}$

In previous work on ion heating and ion flows in the WVU helicon source, ${ }^{8,9,10}$ ion-neutral and electron-neutral collisions have been shown to be important mechanisms for wave damping, flow thermalization, and ion heating. In fact, edge ion heating in cylindrically symmetric plasmas (which is often observed in our experiments and believed to be evidence of damping of edge-localized slow waves in the helicon source $^{11,12}$ ) can also result from the thermalization of ion flow by charge-exchange collisions. ${ }^{13}$ Therefore, although poorly understood, the spatial distribution, temperature, and flow of neutral atoms in helicon sources are important to understanding the physics of helicon sources.

\subsection{Electron beams in helicon sources}

There has been much debate among those in the helicon source community about whether or not an energetic electron beam exists in helicon plasmas. That the efficient ionization of helicon source plasma is due to linear Landau damping by energetic electrons was originally hypothesized by Chen. ${ }^{14}$ However, Chen and Blackwell later discarded this hypothesis because the predicted density of $50 \mathrm{eV}$ electrons would only account for $10 \%$ of the observed ionization, though they do not deny the possible existence of these electrons. ${ }^{15}$ Molvik et al. measured $20 \mathrm{eV}$ electrons using a griddedenergy analyzer. ${ }^{16}$ In this work, we measure the electron population using Langmuir 
probes with RF compensation that prohibits the measurement of any RF-phased pulses of electrons. ${ }^{15}$ To avoid this problem, Chen and Hershkowitz used uncompensated Langmuir probes, and reported measuring electron beams with energies corresponding to phase velocities of multimode helicon waves. ${ }^{17}$ Blackwell and Chen argue that the Chen and Hershkowitz results were still affected by RF potentials, so they made measurements using a time-resolved energy analyzer and reconstructed typical Langmuir probe traces for different phases in the RF cycle. ${ }^{18}$ They reported no evidence of energetic electrons using this method. Recently, Lieberman et al. suggested that an energetic electron beam of $20 \%$ density is required for an electric double layer to form, as has been observed in several helicon source plasmas. ${ }^{19}$

In addition to directly measuring the electrons, the presence of energetic electrons will manifest itself through interactions with the ions and neutrals. Ellingboe et al. found argon ion line emission in a helicon plasma to be modulated at the RF excitation frequency and postulated that the emission modulation was due to pulses of electrons accelerated under the antenna. ${ }^{20}$ They also found that peaks in the emission propagated in phase with the magnetic field. Scharer et al. performed similar experiments, arguing that the measured argon ion line emission could only be present due to $20-45 \mathrm{eV}$ energetic electrons exciting ions into the relevant upper state. ${ }^{21}$ Because the excited states of the neutral atoms are populated by collisions with electrons, the electron dynamics, including the presence of an electron beam, will affect the relative densities of these states. By measuring different excited states, as we are doing with laser-induced fluorescence and passive emission spectroscopy in these experiments, we would expect to see evidence of an electron beam. If the energy of the electron beam were between the energies of the 
two measured states, we would see increased density in the only the lower state, spatially localized similar to the beam. Additionally, for any beam energy, we would expect an electron beam to increase the metastable density, which would increase the density of the nearby states through collisions.

\subsection{Flows and double layers in helicon sources}

Flows are a common phenomenon found in all types of plasmas and can be exhibited by all species, including neutrals. Ions and electrons typically flow along the magnetic field lines. Flow and flow shear can provide an energy source for instabilities and lead to increased particle transport. Ion flow (along the magnetic field) and shear in the ion flow have been measured in the WVU helicon source. ${ }^{22}$ Theoretical studies $^{2}$ suggest such flow shear in the presence of plasmas with thermal anisotropy (as is observed in helicon plasmas ${ }^{23}$ ) may drive ion acoustic and ion cyclotron instabilities in helicon and other laboratory plasmas. ${ }^{24,25,26,27,28,29}$ Hardin et al. have measured bulk rotation about the helicon source axis due to $\mathbf{E x B}$ forces as well as radial diffusion using 3-D LIF measurements. ${ }^{30}$ The bulk rotation of the plasma slows towards the outer edge, which could drive Kelvin-Helmholtz or other perpendicular shear-driven instabilities. These previous studies have focused on the measurement of ion flows. The flows, both radial and rotational, of the neutrals may also drive instabilities, transport, and particle heating. Thus, direct measurements of neutral flows could be important for understanding helicon source physics.

Enhanced axial flows are also of great interest for applications such as thrusters. Recent experiments by a number of groups have demonstrated that electric double layers 
(DLs) can form spontaneously in a current-free plasma expanding in a diverging magnetic field. ${ }^{31,32,33,34,35,36,37}$ Double layers are narrow, local regions of strong electric potential gradient isolated from plasma boundaries. Alfvén suggested that the aurora resulted from energetic electrons precipitating onto the upper atmosphere and that the electrons in space could be accelerated by DL electric fields. ${ }^{38}$ Later observations provided strong experimental evidence in support of Alfvén's hypothesis. ${ }^{39}$ Since then, DLs have been invoked in discussions of solar flare phenomena, ${ }^{40}$ high power gas lasers, ${ }^{41}$ and laser-ablated plasmas. ${ }^{42}$ Previous observations of DLs in space, ${ }^{39}$ in laboratory experiments ${ }^{43,44,45,46,47}$ and simulations ${ }^{48,49}$ have occurred in systems driven by electric current, externally imposed potential differences, or the merging of two plasmas with initially different electron temperatures.

That DLs can form in a current-free plasma expanding in a divergent magnetic field was predicted in an analytical study by Perkins in $1981 .^{50}$ Although experimental observations of ion acceleration in expanding current-free plasmas soon followed, ${ }^{51,52,53}$ no clear evidence of DL formation was obtained in those experiments. Unambiguous observations of DL formation in current-driven plasmas expanding in a diverging magnetic field suggested that divergent magnetic fields themselves could play an important role in initiating DL formation. ${ }^{54}$

Current-free plasma expansion in a divergent magnetic field is surprisingly common and is found on a variety of spatial scales and in a variety of applications. Plasma expansion is essentially equivalent to a pressure gradient arising from a change in the plasma density. The density gradient can give rise to a potential gradient that retards motion of the lighter plasma electrons but accelerates the more massive ions downstream. 
Solar wind expansion and the corresponding creation of the interplanetary electric field is a classic example of this process. ${ }^{55}$ Under isothermal, collisional conditions, the electron density depends on the plasma potential through the classic Boltzmann equation. However, in collisionless plasmas, the mean-free path for ion collisions (such as elastic and charge-exchange collisions) can be much longer than the scale length of the plasma expansion and/or the magnetic field gradient. Under these conditions electric DLs can arise. For DLs that are essentially ion acoustic solitons, the strength of the DL, i.e., the voltage drop across the DL, can be low, a few times the electron thermal energy $\left(\sim 2 k T_{e} / e\right){ }^{56}$ Stronger DLs can be many times the electron temperature, e.g., many hundreds, if not thousands, of times the electron thermal energy. ${ }^{57}$

In the Charles and Boswell experiments, the combination of a strong gradient in the plasma potential and the observation of an ion beam in the diffusion region of the Chi-Kung helicon plasma expanding in a divergent magnetic field demonstrated that a DL was created in the expanding plasma. ${ }^{31}$ Both the plasma potential and ion beam measurements were obtained with a retarding field energy analyzer (RFEA). ${ }^{58}$ In the Cohen et al. ${ }^{34}$ and Sun et al. ${ }^{35}$ expanding helicon plasma experiments, laser induced fluorescence (LIF) measurements of high speed ion flows, i.e., ion beams, were interpreted as evidence of the presence of DLs in the expanding plasmas.

A one-dimensional, unmagnetized, hybrid simulation (particle ions and fluid electrons) that modeled plasma expansion in a diverging magnetic field with a position dependent particle loss rate provided further evidence that a DL can form in a currentfree plasma. ${ }^{59}$ In that simulation, a DL formed at the location of rapid plasma expansion. Throughout the simulation volume, a low energy population of ions created by ionization 
and by charge-exchange collisions was observed. Downstream of the DL, a high-energy ion population accelerated through the DL was observed. The roughly $14 \mathrm{eV}$ potential drop across a DL with a width of a few tens of Debye lengths was obtained in the simulation for an argon plasma at a pressure of $0.5 \mathrm{mTorr}$, an electron density of $6.5 \mathrm{x}$ $10^{8} \mathrm{~cm}^{-3}$, and an electron temperature of $7.2 \mathrm{eV}$. The total ion acceleration occurred over roughly an ion mean-free path.

The hybrid simulation results were consistent with retarding field energy analyzer (RFEA) probe measurements in the Chi-Kung helicon plasma source ${ }^{60}$ that indicated a sharp discontinuity in the plasma potential at the location of rapid plasma expansion ${ }^{31}$ (current-free DL of strength $\sim 3 k T_{e} / e$ ) and the existence of an energetic ion beam downstream of the expansion point $\left(\mathrm{v}_{\text {beam }} \sim 2 \mathrm{v}_{\text {sound }}\right)$ with a density of $\sim 10^{9} \mathrm{~cm}^{-3}$ at low neutral pressures in the source. ${ }^{33}$ Independent experiments in the Magnetic Nozzle Experiment (MNX) reported similar plasma behavior: formation of an energetic, supersonic ion beam below a threshold neutral pressure for a helicon plasma expanding in a divergent magnetic field. ${ }^{34}$

\subsection{Diagnostic development}

To investigate particle flows, heating, and density non-invasively, a multi-species laser-induced fluorescence (LIF) diagnostic was developed at WVU. With the development of diode lasers with adequate power output (see Section 3.1.1) and an Ar II LIF scheme compatible with an existing diode laser wavelength range ${ }^{61}$ Scime and Boivin proposed a diode laser-based diagnostic that could probe Ar II, Ar I, and He I. Compared to a tunable dye laser-based system, the diode laser-based diagnostic is less 
expensive, easier to operate, and portable.

To accomplish three different LIF schemes with one diode laser, the schemes for Ar I and He I had to be somewhat unconventional. The Ar I scheme does not begin with a metastable state, as most LIF schemes do, to ensure an adequate state population for the diagnostic. Thus, the initial state must be populated by collisions. The He I scheme requires a collisional transfer after the laser excitation to yield fluorescence at a wavelength that can be easily detected. Thus, both of these schemes require higher plasma pressures to provide the necessary collisions.

Boivin reproduced the Ar II scheme with the diode laser in the plasma chamber at WVU. He also successfully performed initial measurements with the He I scheme. ${ }^{62} \mathrm{My}$ contribution to the development of diode laser-based LIF measurements in argon was to successfully demonstrate the $\mathrm{Ar} I$ scheme,${ }^{63}$ satisfying the intended measurement objectives of our diagnostic. The portability of the diagnostic was demonstrated during experiments on the Magnetic Nozzle Experiment at Princeton Plasma Physics Lab ${ }^{34}$ and the Chi-Kung Experiment at Australian National University. ${ }^{64}$

\subsection{Neutral argon line structure}

To use optical spectroscopy diagnostics, the energy level structure of the target species must be understood. While most energy levels can be described by understanding Russell-Saunders coupling of the electrons, there are other coupling mechanisms that yield more complex level-naming systems. For Ar I, jj-coupling must be used to describe the sublevels because the interaction between the spin of the outermost electron and its own orbit is greater than the interactions between the spins of the electron and parent ion 
and between the orbits of the electron and parent ion.

The most common interaction between two electrons is Russell-Saunders coupling, also called L-S coupling. Each electron has an orbital angular momentum $\mathbf{l}_{\mathrm{i}}$ and a spin angular momentum $\mathbf{s}_{\mathrm{i}}$. The interaction energy of the spins of each electron and that of the orbital momenta is greater than the interaction energy between an electron's spin and its orbital momentum, so that the $\mathbf{s}_{\mathrm{i}}$ 's combine to create the total spin momentum $\mathbf{S}$, where $\left|s_{1}-S_{2}\right| \leq S \leq s_{1}+s_{2}$ and the $\mathbf{l}_{\mathrm{i}}$ 's combine to create the total orbital momentum $\mathbf{L}$, where $\left|l_{1}-l_{2}\right| \leq L \leq l_{1}+l_{2}$. The total angular momentum $\mathbf{J}$ is then obtained from combining $\mathbf{L}$ and $\mathbf{S}$, where $|L-S| \leq J \leq L+S$. While the interaction energy between the spins is generally thought of as due to the magnetic moments of the electrons, it is actually due to a phenomenon known as the Heisenberg-Dirac resonance caused by the charges of the electrons. ${ }^{65}$ The interaction between the orbital momenta is strong because the orbits of the electrons overlap, causing them to perturb each other.

There are additional ways for electrons to interact with each other besides L-S coupling. One of these possibilities is $\mathrm{j}-\mathrm{j}$ coupling. In this case, the interaction energy between the spin and orbital momenta of an electron is greater than that between the spins of the two electrons. In this case, the $\mathbf{~}_{\mathrm{i}}$ and $\mathbf{s}_{\mathrm{i}}$ combine to form a $\mathbf{j}_{\mathrm{i}}$, where $\left|l_{i}-S_{i}\right| \leq j_{i} \leq$ $l_{i}+s_{i}$. The total angular momentum $J$, is then obtained by combining the $\mathbf{j}_{\mathrm{i}}$ 's, with $\left|j_{1}-j_{2}\right| \leq J$ $\leq j_{1}+j_{2}$. The vector models for L-S and $\mathrm{j}-\mathrm{j}$ coupling are shown in Figure 1.1. ${ }^{66}$ The vector magnitude $J^{*}$ is given by $\sqrt{J(J+1)}$, and similarly for the other vectors. For L-S coupling, the $\mathbf{l}_{\mathrm{i}}$ 's and $\mathbf{s}_{\mathrm{i}}$ 's precess about their resultant $\mathbf{L}$ and $\mathbf{S}$, respectively. The $\mathbf{L}$ and $\mathbf{S}$, then precess around the resultant $\mathbf{J}$. For $\mathrm{j}-\mathrm{j}$ coupling, the $\mathbf{l}_{\mathrm{i}}$ and $\mathbf{s}_{\mathrm{i}}$ precess about their resultant $\mathbf{j}_{\mathrm{i}}$, and the $\mathbf{j}_{\mathrm{i}}$ 's precess about the resultant $\mathbf{J}$. 

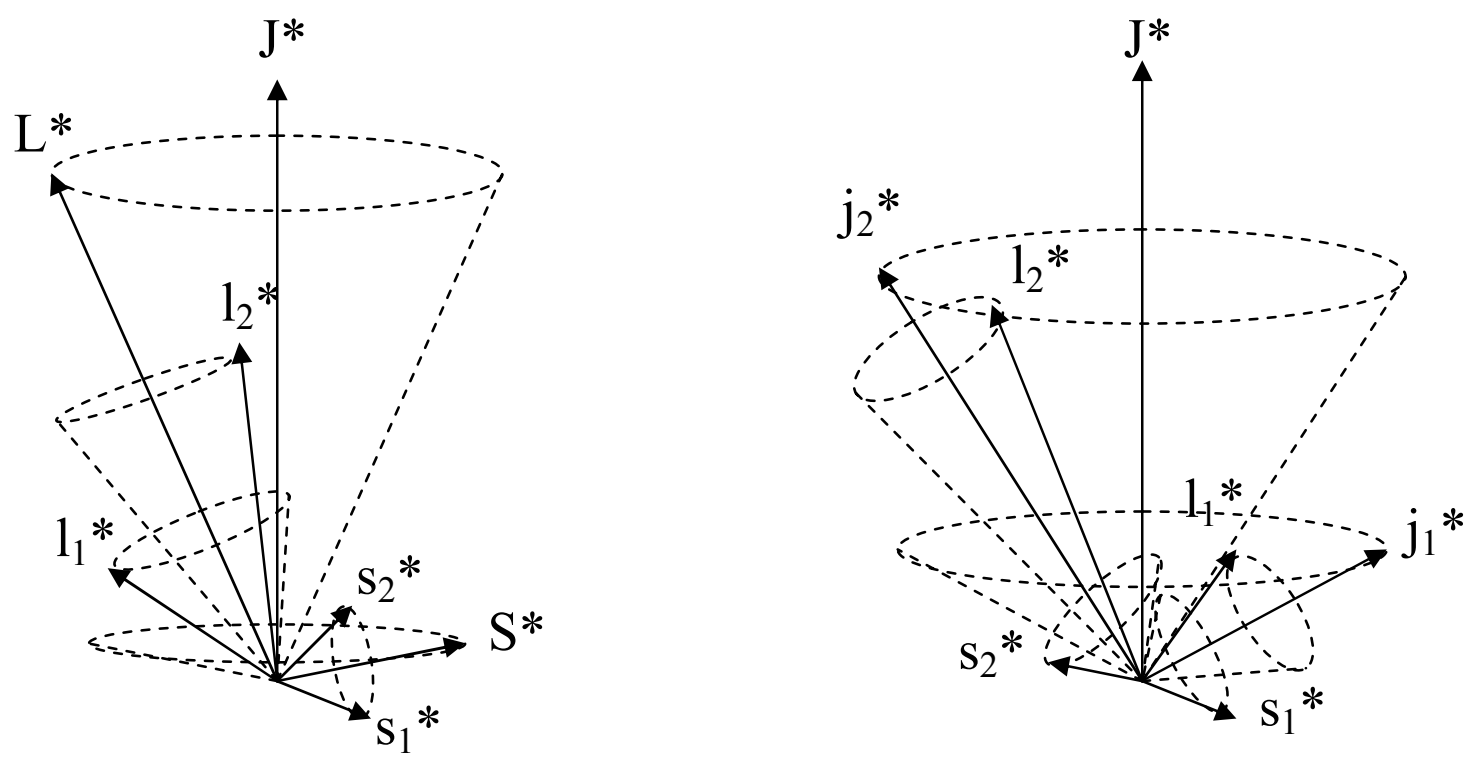

Figure 1.1 Vector model for L-S (left) and j-j (right) coupling. The vector magnitudes are given by $J^{*}=\sqrt{J(J+1)}$.

These coupling schemes can be extended to entire atoms, where the outermost electron couples with the remaining constituents of the atom. This remaining part of the atom is often referred to as the "parent ion" since removal of the outermost electron would yield an ion with the same configuration. In L-S coupling, the total $\mathbf{s}$ of the parent ion couples with the $\mathbf{s}_{\mathrm{i}}$ of the electron to create the total $\mathbf{S}$ of the atom. Similarly, the parent's $\mathbf{I}$ and the electron's $\mathbf{I}_{\mathbf{i}}$ combine to create the total $\mathbf{L}$ of the atom. Then the $\mathbf{L}$ and $\mathbf{S}$ combine to give the total $\mathbf{J}$ of the atom. This is similar for $\mathbf{j}-\mathbf{j}$ coupling, where the total $\mathbf{j}$ of the parent ion couples with the $\mathbf{j}_{\mathrm{i}}$ of the electron to obtain the atom's total $\mathbf{J}$. (This is sometimes written as $\mathrm{J}-\mathrm{j}$ coupling.) The method of coupling used to determine the parent's $\mathbf{L}, \mathbf{S}$, and $\mathbf{J}$ is inconsequential.

Each energy level will have a value known as the Landé $g$-factor, which is a ratio of the atom's magnetic moment to its mechanical moment. ${ }^{65}$ Because the $g$-factor is 
dependent on the angles between the vectors, it will be different depending on the method of coupling. For L-S coupling,

$$
g=1+\frac{J(J+1)+S(S+1)-L(L+1)}{2 J(J+1)},
$$

while for $\mathrm{j}-\mathrm{j}$ coupling,

$$
g=g_{1} \frac{J(J+1)+j_{1}\left(j_{1}+1\right)-j_{2}\left(j_{2}+1\right)}{2 J(J+1)}+g_{2} \frac{J(J+1)+j_{2}\left(j_{2}+1\right)-j_{1}\left(j_{1}+1\right)}{2 J(J+1)},
$$

where $g_{1}$ and $g_{2}$ are the $g$ values for the first and second electrons, respectively, calculated using the former equation for $g .{ }^{65}$

When the atom is placed in a magnetic field of magnitude $H$, the magnetic moment of the atom will interact with the field. In the vector model, $\mathbf{J}$ will precess about $\mathbf{H}$, and the projection of $\mathbf{J}$ onto $\mathbf{H}$ yields the magnetic angular momentum quantum number, $\mathbf{M}$, where $-J \leq M \leq J$. This removal of degeneracy when placed in a magnetic field is called the (anomalous) Zeeman effect. Figure 1.2 shows the vector model for L-S and $\mathrm{j}$-j coupling in a magnetic field. ${ }^{66}$ As shown in the figure, the projection of $\mathbf{J}^{*}$ onto $\mathbf{H}$ will have the magnitude $M g$, where $g$ is the Landé $g$-factor. This applies for fields that are not strong enough to overcome the interactions between the $\mathbf{l}_{\mathrm{i}}$ 's and $\mathbf{s}_{\mathrm{i}}$ 's and the $\mathbf{S}$ and $\mathbf{J}$ for L-S coupling, or between the $\mathbf{l}_{\mathrm{i}}$ 's, the $\mathbf{s}_{\mathrm{i}}$ 's, and the $\mathbf{j}_{\mathbf{i}}$ 's for $\mathrm{j}$-j coupling. Stronger fields can perturb the original interactions, leading to the Paschen-Back effect, which is not relevant for our application. 

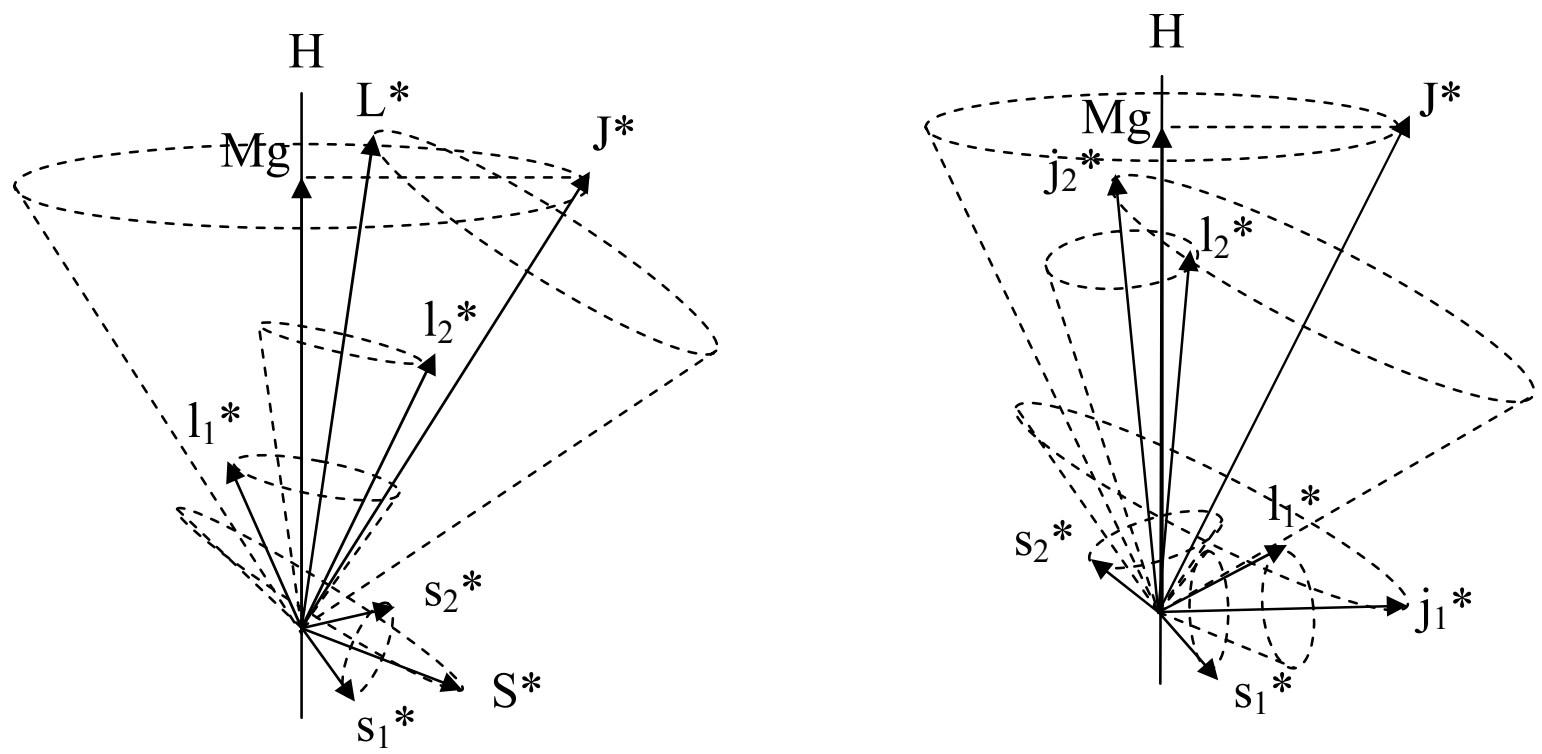

Figure 1.2 Vector model for L-S (left) and j-j (right) coupling in a weak magnetic field. The vector magnitudes are given by $J^{*}=\sqrt{J(J+1)}$.

Because most excited states of the noble gases don't follow L-S coupling, the usual notation, ${ }^{2 S+1} L_{J}$ cannot be used. Several types of notation can be found describing the states of noble gases, including Paschen notation, Racah notation, and j-j notation.

When Paschen studied the spectrum of neon, he found that he could arrange the lines into four series of $s$ terms, ten series of $p$ terms, twelve series of $d$ terms, and twelve series of $f$ terms using Rydberg's formulas. ${ }^{66}$ Further understanding of the atomic structure and Rydberg formula indicated that a series corresponds to all radiative transitions to a single energy state. Thus, there are four $s$ states (i.e. the outermost electron is in an $s$ state), ten $p$ states, etc. The four $s$ states are the first excited states and are notated $1 s_{2}, 1 s_{3}, 1 s_{4}, 1 s_{5}$. The next excited states are $p$ states, notated $2 p_{1}$ through $2 p_{10}$. The notation continues in this manner for higher excited states. The ground state is notated $1 p_{0}$ because all six electrons in the outer shell are $p$ electrons. This notation can 
be extended to the other noble gases, including argon.

Racah notation has the format $n l[K]_{J}$ where $n$ is the principal quantum number of the outer electron, $l$ is the orbital angular momentum quantum number of the outer electron, $K$ is the sum of $l$ and the total angular momentum of the parent ion, and $J$ is the total angular momentum of the atom obtained by adding $K$ and $S .{ }^{67}$ The prime indicates that the parent ion has a total angular momentum of $1 / 2$.

It is also possible to find the states written with a notation that explicitly indicates the $\mathrm{j}$ - $\mathrm{j}$ coupling. This notation is in the form $\left(j_{1} j_{2}\right)_{J}$ where each term is defined as in the description of j-j coupling above. ${ }^{66}$

The ground state of argon is given by $1 s^{2} 2 s^{2} 2 p^{6} 3 s^{2} 3 p^{6}{ }^{1} S_{0}$. Because argon contains a complete p-group, as do all noble gases, singly ionized argon yields an inverted ${ }^{2} \mathrm{P}$ doublet. ${ }^{66}$ For an excited argon atom, the outer electron couples with the parent ion via Russell-Saunders coupling if it is in one of the lower $s$ states. If the outer electron is in a higher state, it couples with the parent ion according to $\mathrm{j}-\mathrm{j}$ coupling. ${ }^{65}$ Table 1.1 shows the different notation schemes for the first 15 states of argon neutrals and their energies. For the work described here, we will use Racah notation to describe the excited argon neutral states. 
Table 1.1 Argon state notation and energies

\begin{tabular}{|l|l|l|l|l|}
\hline Paschen & $\begin{array}{l}\mathrm{J}-\mathrm{L} \\
\text { Coupling } \\
(\text { Racah })\end{array}$ & $\begin{array}{l}\text { L-S } \\
\text { Coupling }\end{array}$ & Energy (eV) & \\
\hline $1 \mathrm{p}_{0}$ & & ${ }^{\mathrm{I}} \mathrm{S}_{0}$ & & Ground \\
\hline $1 \mathrm{~s}_{5}$ & $4 \mathrm{~s}[3 / 2]^{\circ}{ }^{\circ}$ & ${ }^{3} \mathrm{P}_{2}$ & 11.55 & Metastable \\
\hline $1 \mathrm{~s}_{4}$ & $4 \mathrm{~s}[3 / 2]^{\circ}{ }_{1}$ & ${ }^{3} \mathrm{P}_{1}$ & 11.62 & \\
\hline $1 \mathrm{~s}_{3}$ & $4 \mathrm{~s}^{\prime}[1 / 2]^{\circ}{ }_{0}$ & ${ }^{3} \mathrm{P}_{0}$ & 11.72 & Metastable \\
\hline $1 \mathrm{~s}_{2}$ & $4 \mathrm{~s}^{\prime}[1 / 2]_{1}^{\circ}$ & ${ }^{\mathrm{I}} \mathrm{P}_{1}$ & 11.82 & \\
\hline $2 \mathrm{p}_{10}$ & $4 \mathrm{p}[1 / 2]_{1}$ & & 12.91 & \\
\hline $2 \mathrm{p}_{9}$ & $4 \mathrm{p}[5 / 2]_{3}$ & & 13.08 & \\
\hline $2 \mathrm{p}_{8}$ & $4 \mathrm{p}[5 / 2]_{2}$ & & 13.10 & \\
\hline $2 \mathrm{p}_{7}$ & $4 \mathrm{p}[3 / 2]_{1}$ & & 13.15 & \\
\hline $2 \mathrm{p}_{6}$ & $4 \mathrm{p}[3 / 2]_{2}$ & & 13.17 & \\
\hline $2 \mathrm{p}_{5}$ & $4 \mathrm{p}[1 / 2]_{0}$ & & 13.27 & \\
\hline $2 \mathrm{p}_{4}$ & $4 \mathrm{p}^{\prime}[3 / 2]_{1}$ & & 13.28 & \\
\hline $2 \mathrm{p}_{3}$ & $4 \mathrm{p}^{\prime}[3 / 2]_{2}$ & & 13.30 & \\
\hline $2 \mathrm{p}_{2}$ & $4 \mathrm{p}^{\prime}[1 / 2]_{1}$ & & 13.33 & \\
\hline $2 \mathrm{p}_{1}$ & $4 \mathrm{p}^{\prime}[1 / 2]_{0}$ & & 13.48 & \\
\hline
\end{tabular}




\section{References}

${ }^{1}$ G. R. Tynan, J. Appl. Phys. 86, 5356 (1999).

${ }^{2}$ C. Watts and J. Hanna, Phys. Plasmas 11, 1358 (2004).

${ }^{3}$ R. W. Boswell and K. Porteous, Appl. Phys. Lett. 50, 1130 (1987).

${ }^{4}$ S.A. Hokin, et al., Nuclear Fusion 37, 1615 (1997).

${ }^{5}$ J. Gilland, R. Breun, and N. Hershkowitz, Plasma Sources Sci. Technol. 7, 416 (1998).

${ }^{6}$ A. W. Degeling, T. E. Sheridan, and R. W. Boswell, Phys. Plasmas 6, 1641 (1999).

${ }^{7}$ M. Yoon, S. C. Kim, H. J. Lee, et al., J. Korean Phys. Soc. 32, L635 (1998).

${ }^{8}$ J. L. Kline, E. E. Scime, P.A. Keiter, M. M. Balkey, and R. F. Boivin, Phys. Plasmas 6, 4767 (1999).

${ }^{9}$ E.E. Scime, P.A. Keiter, M.W. Zintl, M.M. Balkey, J.L. Kline, and M.E. Koepke, Plasma Sources Sci. Technol. 7, 186 (1998).

${ }^{10}$ J. L. Kline, M. Balkey, C. Compton, R. Boivin, R. Hardin, A. M. Keesee, P. Keiter, E. Scime, and X. Sun, Phys. Plasmas 10, 2127 (2003).

${ }^{11}$ J. Kline, E. Scime, R. Boivin, A. M. Keesee, X. Sun, and V. Mikhailenko, Phys. Rev. Lett. 88, (2002).

12 J. L. Kline, E. Scime, R. Boivin, A. M. Keesee, and X. Sun, Plasma Sources Sci. Technol. 11, 413 (2002).

${ }^{13}$ F. Anderegg, R.A. Stern, F. Skiff, B.A. Hammel, M.Q. Tran, P.J. Paris, and P. Kohler, Phys. Rev. Lett. 57, 329 (1986).

${ }^{14}$ F.F. Chen, Plasma Phys. Controlled Fusion 33, 339 (1991).

${ }^{15}$ F.F. Chen and D.D. Blackwell, Phys. Rev Lett. 82, 2677 (1999).

${ }^{16}$ A.W. Molvik, A.R. Ellingboe, and T.D Rognlien, Phys. Rev. Lett. 79, 233 (1997).

${ }^{17}$ R.T.S. Chen and N. Hershkowitz, Phys. Rev. Lett. 80, 4677 (1998).

${ }^{18}$ D. D. Blackwell and F.F. Chen, Plasma Sourc. Sci. Technol. 10, 226 (2001).

${ }^{19}$ M.A. Lieberman, C. Charles, and R.W. Boswell, J. Phys. D: Appl. Phys. 39, 3294 (2006).

${ }^{20}$ A.R. Ellingboe, R.W. Boswell, J.P. Booth, and N. Sadeghi, Phys. Plasmas 2, 1807 (1995).

${ }^{21}$ J. Scharer, A. Degeling, G. Borg, and R. Boswell, Phys. Plasmas 9, 3734 (2002).

${ }^{22}$ X. Sun, A. Keesee, J. Kline, and E. E. Scime, Bull. Am. Phys. Soc. 47, 32 (2002).

${ }^{23}$ E.E. Scime, P.A. Keiter, M.W. Zintl, M.M. Balkey, J.L. Kline, and M.E. Koepke, Plasma Sources Sci. Technol. 7, 186 (1998).

${ }^{24}$ V. V. Gavrishchaka, G. I. Ganguli, S. B. Ganguli, Phys. Rev. Lett. 80, 728 (1998).

${ }^{25}$ V. V. Gavrishchaka, S. B. Ganguli, G. I. Ganguli, J. Geophys. Res. 104, 12,683 (1999).

${ }^{26}$ G. Ganguli, M. J. Keskinen, H. Romero, R. Heelis, T. Moore, C. Pollock, J. Geophys. Res. 99, 8873 (1994).

${ }^{27}$ G. Ganguli, Y. C. Lee, P. J. Palmadesso, Phys. Fluids 31, 823 (1988).

${ }^{28}$ E.E. Scime, A. M. Keesee, R. S. Spangler, M. E. Koepke, C. Teodorescu, and E. W. Reynolds, Phys Plasmas 9, 4399 (2002).

${ }^{29}$ M.E. Koepke, C. Teodorescu, and E.W. Reynolds, Plasma Phys. Control. Fusion 45, 869 (2003).

${ }^{30}$ R. Hardin, X. Sun, and E. Scime, Rev. Sci. Instrum. 75, 4103 (2004).

${ }^{31}$ C. Charles and R.W. Boswell, Appl. Phys. Lett. 82,1356 (2003).

${ }^{32}$ C. Charles, Appl. Phys. Lett. 84, 332 (2004).

${ }^{33}$ C. Charles and R.W. Boswell, Phys. Plasmas 11, 3808 (2004).

${ }^{34}$ S. A. Cohen, N.S. Siefert, S. Stange, E.E. Scime, R. F. Boivin, and F. Levinton, Phys. Plasmas 10, 2593 (2003).

${ }^{35}$ X. Sun, C. Biloiu, R. Hardin, and E. Scime, Plasma Sources Sci. Technol. 13, 359 (2004).

${ }^{36}$ X. Sun, S.A. Cohen, E.E. Scime, and M. Miah, Phys. Plasmas 12, 103509 (2005).

${ }^{37}$ S.A. Cohen et al., IEEE Trans.Plasma Sci. 34, 792 (2006).

${ }^{38}$ H. Alfven, Tellus 10, 104 (1958).

${ }^{39}$ R. D. Albert and P. J. Lindstrom, Science 170, 1398 (1970). 
${ }^{40}$ P. Carlqvist, in Proceedings of the Second Symposium on Plasma Double-layers and Related Topics, edited by R. Schrittweiser and G. Eder (Institute for Theoretical Physics, Innsbruck, Austria, 1984), p. 340 .

${ }^{41}$ W. B. Bridges et al., Proc. IEEE 59, 724 (1971).

${ }^{42}$ H. Hora, P. Lalousis, and S. Eliezer, Phys. Rev. Lett. 53, 1650 (1984).

${ }^{43}$ P. Coakley and N. Hershkowitz, Phys. Fluids 22, 1171 (1979).

${ }^{44}$ B. H. Quon and A.Y. Wong, Phys. Rev. Lett. 37, 1393 (1976).

${ }^{45}$ S. Torvén and D. Anderson, J. Phys. D. 12, 717 (1979).

${ }^{46}$ R.L. Stenzel, M. Ooyama, and Y. Nakamura, Phys. Rev. Lett. 45, 86 (1980).

${ }^{47}$ M.J. Alport, S.L. Cartier, and R.L. Merlino, J. Geophys. Res. 91, 1599 (1986).

${ }^{48}$ C. K. Goertz and G. Joyce, Astrophys. Space Sci. 32, 165 (1975).

${ }^{49}$ D. L. Newman et al., Phys. Rev. Lett. 87, 255001 (2001).

${ }^{50}$ F.W. Perkins and Y. C. Sun, Phys. Rev. Lett. 46, 115 (1981).

${ }^{51}$ D. J. Trevor et al., Appl. Phys. Lett. 57, 1188 (1990).

${ }^{52}$ N. Sadeghi et al., J. Appl. Phys. 70, 2552 (1991).

${ }^{53}$ C. Charles et al., J. Vac. Sci. Technol. A 9, 661 (1991).

${ }^{54}$ R. Schrittwieser et al., IEEE Trans. Plasma Sci. 20, 607 (1992).

${ }^{55}$ E. N. Parker, J. Geophys. Res. 62, 509 (1957).

${ }^{56}$ C. Chan, M. H. Cho, N. Hershkowitz, and T. Intrator, Phys. Rev. Lett. 52, 1782 (1984).

${ }^{57}$ Y. Takeda and K. Yamagiwa, Phys. Rev. Lett. 55, 711 (1985).

${ }^{58}$ C. Charles, A.W. Degeling, T.E. Sheridan, J.H. Harris, M.A. Lieberman, and R.W. Boswell, Phys. Plasmas 7, 5232 (2000).

${ }^{59}$ A. Meige et al., IEEE Trans. Plasma Sci. 33, 334 (2005).

${ }^{60}$ C. Charles et al., Phys. Plasmas 7, 5232 (2000).

${ }^{61}$ G.D. Severn, D.A. Edrich, and R. McWilliams, Rev. Sci. Instrum. 69, 10 (1998).

${ }^{62}$ R.F. Boivin and E.E. Scime, Rev. Sci. Instrum. 74, 4352 (2003).

${ }^{63}$ A.M. Keesee, E.E. Scime, and R.F. Boivin, Rev. Sci. Instrum. 75, 4091 (2004).

${ }^{64}$ A.M. Keesee, E.E. Scime, C. Charles, A. Meige, and R. Boswell, Phys. Plasmas 12, 93502 (2005).

${ }^{65}$ L. Pauling and S. Goudsmit, The Structure of Line Spectra, (New York, 1930).

${ }^{66} \mathrm{H}$. White, Introduction to Atomic Spectra, (New York, 1934).

${ }^{67} \mathrm{http}: / /$ arxiv.org/pdf/physics/0005082 


\section{Chapter 2: Experimental Setup}

The combination of the Hot hELIcon eXperiment (HELIX) and the Large Experiment on Instabilities and Anisotropies (LEIA) was originally designed to study magnetospherically relevant plasmas. The plasma is created in the HELIX plasma source and flows into the larger LEIA chamber where the magnetic field is weaker, allowing for high beta $\left(\beta=n k_{B} T \mu_{0} / B^{2}\right)$ plasmas. The geometry of the system also enables studies relevant to plasma processing and space propulsion. A picture of HELIX and LEIA is shown in Figure 2.1.

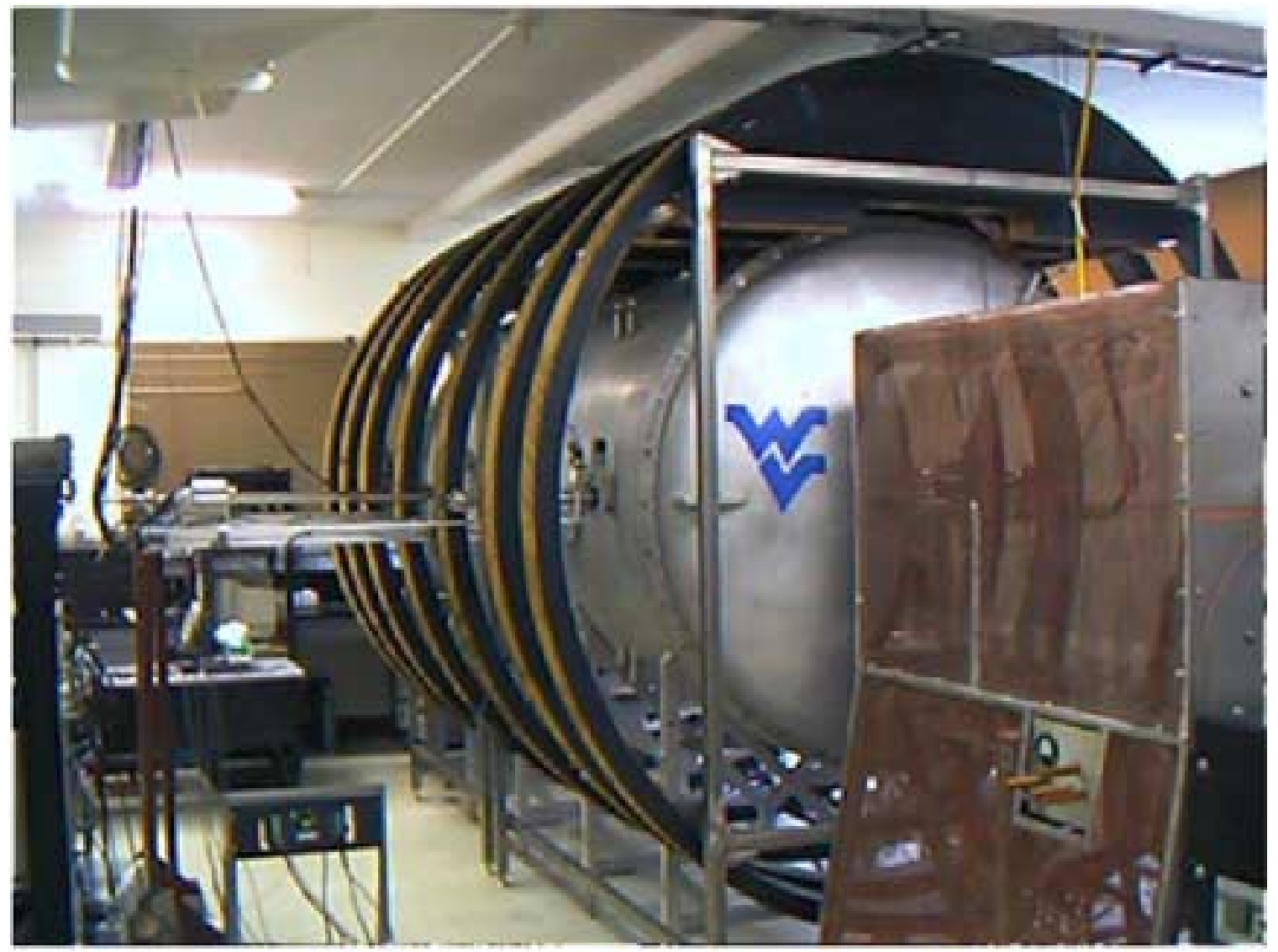

Figure 2.1 HELIX (foreground) and LEIA combined system. HELIX is enclosed in a copper Faraday cage. 
The experimental hardware used in this work is described in this chapter. All the measurements (except those described in Chapter 4) were performed in HELIX, while LEIA parameters were held fixed. Additional descriptions of the experimental apparatus can be found in Refs. [1,2].

\subsection{Plasma chamber}

The HELIX vacuum chamber is a $61 \mathrm{~cm}$ long, Pyrex tube $10 \mathrm{~cm}$ in diameter connected to a $91 \mathrm{~cm}$ long, $15 \mathrm{~cm}$ diameter, stainless steel chamber. The chamber has one set of four 6" Conflat ${ }^{\mathrm{TM}}$ crossing ports in the center of the chamber and four sets of four 2 3/4" Conflat ${ }^{\mathrm{TM}}$ crossing ports on either side that are used for diagnostic access. The four 6" crossing ports are fitted with 4" viewports for optical diagnostics. The end of the stainless steel chamber is connected to LEIA, a $1.8 \mathrm{~m}$ diameter, $4.4 \mathrm{~m}$ long space chamber. The end of the LEIA chamber is connected to a pumping station. At the opposite end, the HELIX chamber is connected to a glass tee with each branch terminated by the pumping station, ion gauge, and 12" stainless steel flange fitted with a 4" viewport, respectively. A schematic of the HELIX and LEIA system is shown in Figure 2.2. The $z$-axis is measured from the junction of the HELIX chamber and glass tee. 


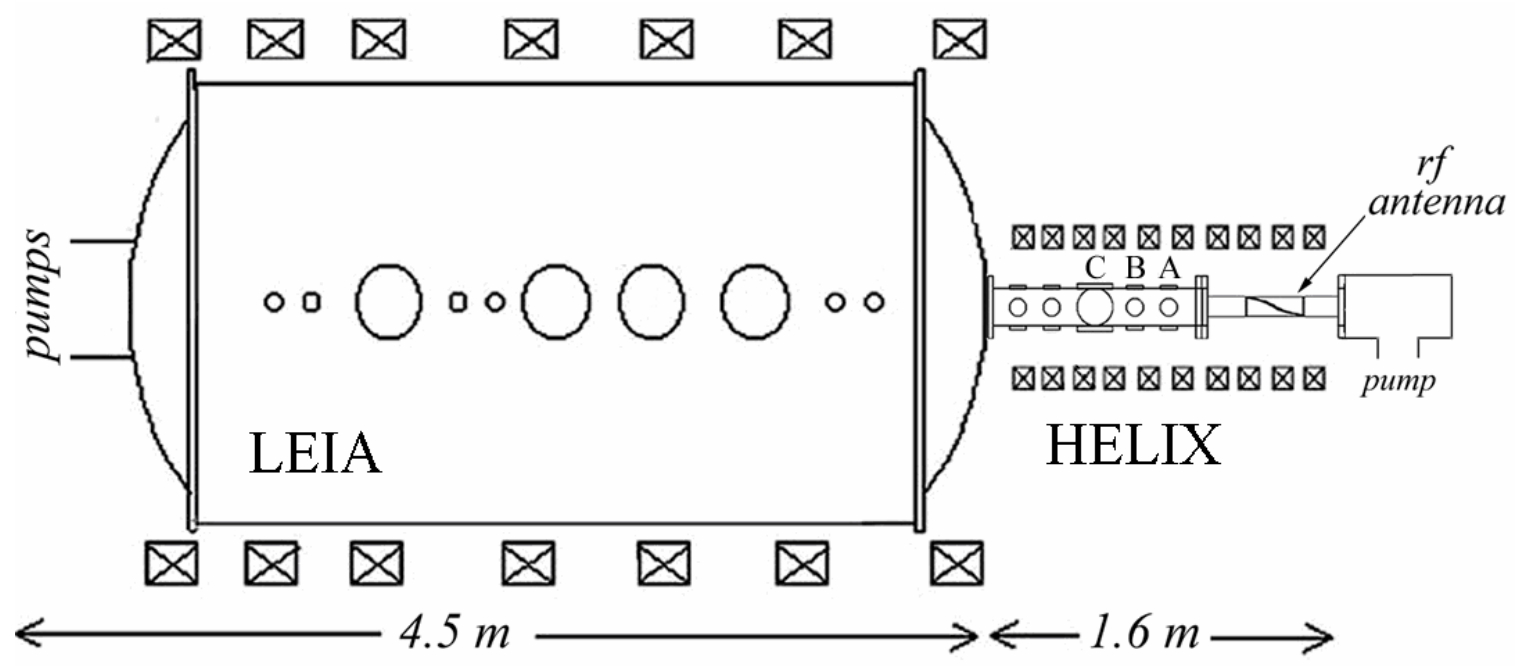

Figure 2.2 Schematic diagram of the HELIX and LEIA chambers. The locations of the A) Baratron gauge $(\mathrm{z}=80 \mathrm{~cm}), \mathrm{B})$ Langmuir probe $(\mathrm{z}=95 \mathrm{~cm})$, and $\mathrm{C})$ optical diagnostics $(\mathrm{z}=110 \mathrm{~cm})$ collection are shown.

At location C (the 6" crossing ports) in Figure 2.2, a scanning stage for optical diagnostics collection is mounted to the chamber. A schematic of the stage is shown in Figure 2.3. The stage consists of two Velmex motor driven Unislide rail assemblies, one horizontal and one vertical. A set of collection optics is attached to each rail. The computer controlled stepping motor allows for precise positioning of the optics and scanning of the optics across the viewport. Typically, the optics on the vertical scanning stage are collimated while the optics attached to the horizontal scanning stage are focused such that the focal point intersects the line of sight of the collimated optics. When scanning vertically, the entire stage moves, such that the focal point always intersects the line of sight of the collimated optics. The viewing area is an $8 \mathrm{~cm} \times 8 \mathrm{~cm}$ plane perpendicular to the chamber axis, limited primarily by the size of the viewports. 


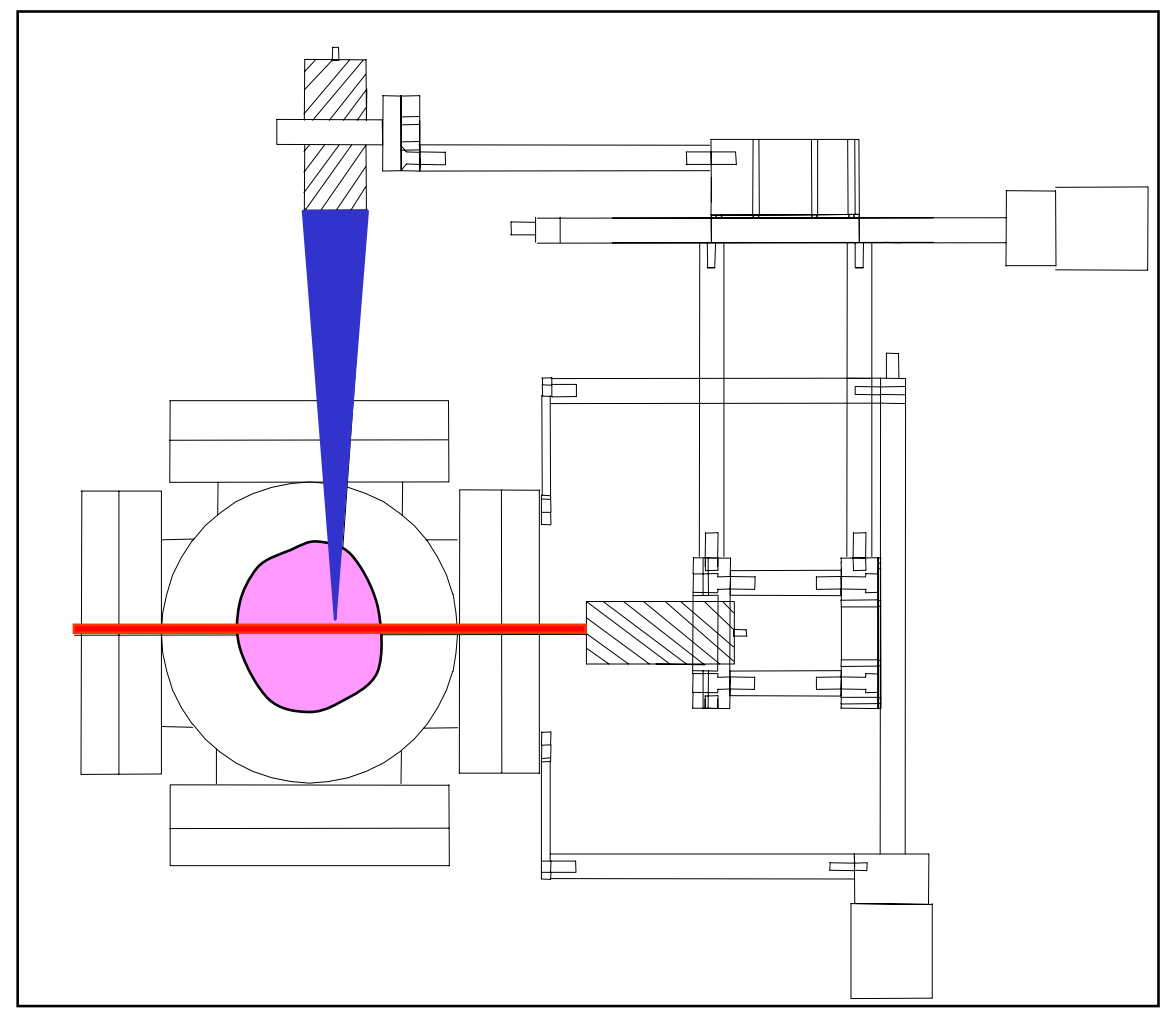

Figure 2.3 Optical diagnostics collection scanning stage

The $x$-axis used in the measurements is defined by the horizontal Velmex stage such that $x=0$ corresponds to $r=0$ (the axis of the plasma chamber), and $x>0$ to the left in Figure 2.3 (closest to the exterior window in the lab). Similarly, the $y$-axis is defined by the vertical Velmex stage such that $y=0$ corresponds to $r=0$ (the axis of the plasma chamber), and $y>0$ as the stage scans upward. For any horizontal scan with $y=0$ fixed, the $|x|$ value will correspond to $r$ such that this will provide a radial scan. This is only true for a horizontal scan using the focused optics.

\subsection{Vacuum system}

The vacuum pressure in the chamber is maintained by a set of three turbomolecular drag pumps, each backed by a diaphragm roughing pump. A Balzers 
TMU 520 turbomolecular drag pump is connected to one branch of the glass tee at the end of HELIX. A Balzers MD 4T diaphragm roughing pump is attached to the turbo pump. A MDC GV-4000M-P 6 inch inner diameter gate valve is located between the turbo pump and the glass tee. Two Pfeiffer TMU 1600 turbomolecular pumps are connected to the end of the LEIA chamber. Each is connected to a Pfeiffer MD-8 diaphragm roughing pump. Two MDC GV-8000V-P 10 inch gate valves separate the turbo pumps from the LEIA chamber.

The three pumps maintain a base pressure on the order of $10^{-7}$ Torr. An interlock system is used to shut the gate valves in the event of a high pressure reading or loss of power. The pressure is measured by two Balzers PKR250 full range pressure gauges with Balzers TPG252 dual gauge controllers. The gauges achieve full range by combining a Pirani gauge for pressure above $10^{-2}$ Torr and a cold cathode gauge for pressures below $10^{-2}$ Torr. $^{3}$ The pressure gauge for the HELIX pumping station is located on one branch of the glass tee. The gauge for the LEIA pumping station is located on LEIA, toward the pumping station end of the chamber. A MKS1179 mass flow valve with a PR-4000 flow controller regulates the gas flow to maintain the desired neutral pressure. A Baratron ${ }^{\circledR}$ capacitance manometer pressure gauge is located $35 \mathrm{~cm}$ downstream of the antenna (location A in Figure 2.2) for additional pressure readings. The Baratron gauge reading is independent of gas species. Neutral pressures from 0.1 to 100 mTorr are achievable.

Because the Balzers PKR250 pressure gauges are calibrated for nitrogen gas, they must be calibrated separately for argon and helium. The Balzers gauges were calibrated to the Baratron gauge by Compton and Biloiu. To determine the conversion from gauge reading to actual pressure for argon, the gate valves were closed, vacuum pumps were 
turned off, and a puff of argon gas (flow of $5 \mathrm{sccm}$ for a short time) was introduced into the chamber. The gas was allowed to equilibrate and the pressure was assumed to be uniform throughout the entire system. Then readings from the front (HELIX) Balzers, Baratron located at $z=220 \mathrm{~cm}$ (at the second large port in LEIA), and back (LEIA) Balzers gauges were recorded. Compton and Biloiu gradually increased the pressure by introducing additional gas puffs. Calibration curves measured by Compton and Biloiu with no plasma discharge are shown in Figure 2.4 and Figure 2.5. There are different calibration curves for $P<4$ mTorr and $P>4$ mTorr due to the switching of the Balzers gauge from cold cathode to Pirani gauge. (There may have been some confusion in pressure conversion that resulted in the choice of 4 mTorr as a breakpoint. The Balzers gauge reading and actual pressure agree at $4 \times 10^{-3}$ mbar, with differing characteristics above and below this value due to the different gauges. ${ }^{3} 4 \times 10^{-3}$ mbar is equivalent to a pressure of $\sim 3$ mTorr, which probably should have been the breakpoint used for the calibration data.)
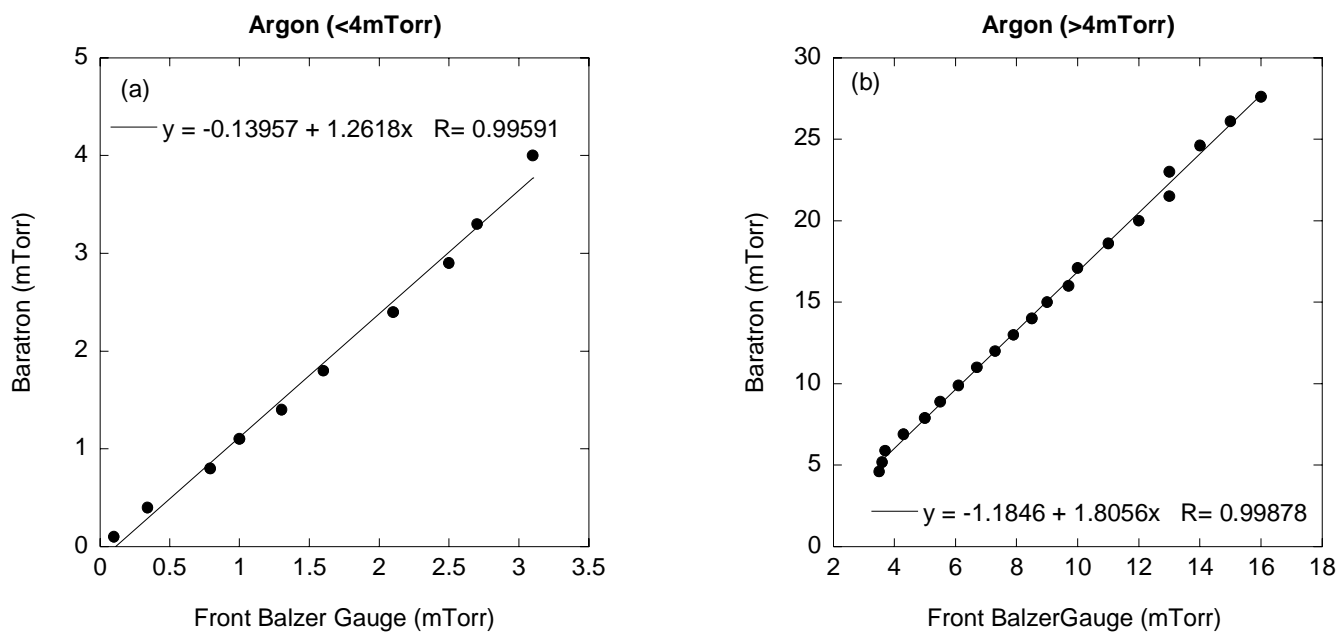

Figure 2.4 Pressure gauge calibration for front (HELIX) Balzers gauge using Baratron gauge for pressures a) $<4$ mTorr and b) $>4 \mathrm{mT}$ Torr. The linear fit shown is used to convert the gauge pressure reading to actual pressure. 

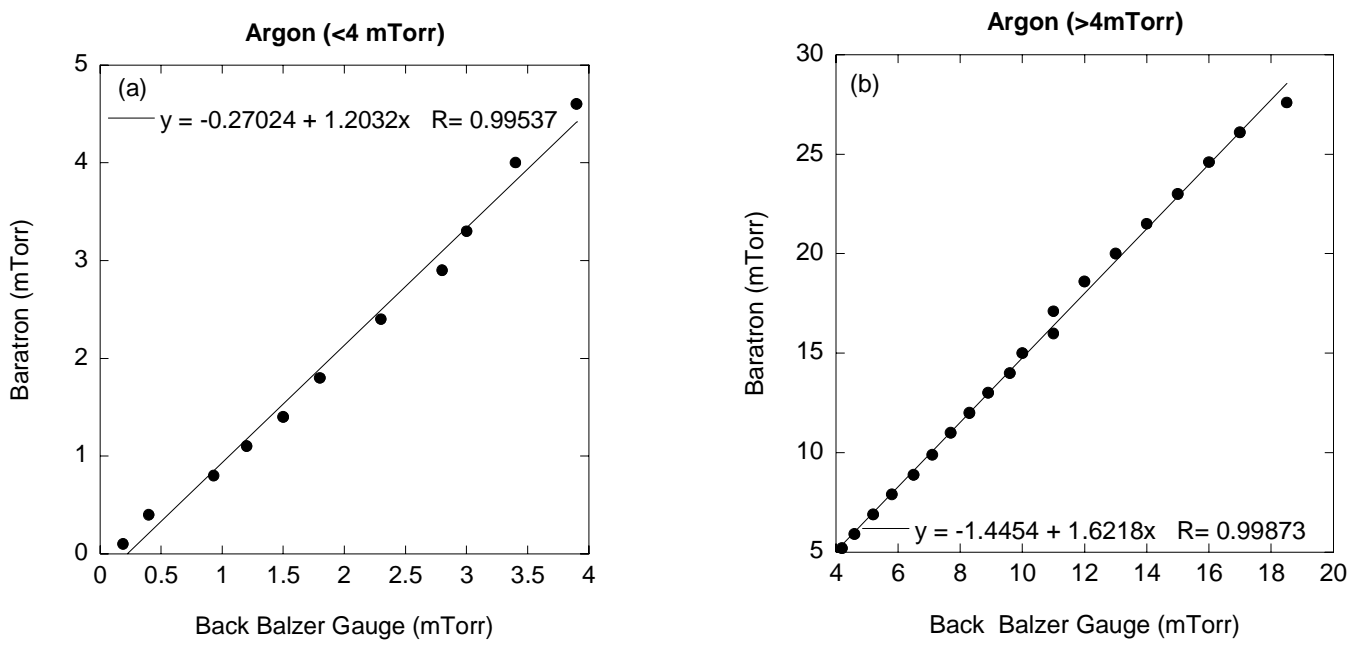

Figure 2.5 Pressure gauge calibration for back (LEIA) Balzers gauge using Baratron gauge for pressures a) $<4 \mathrm{mTorr}$ and b) $>4 \mathrm{mTorr}$. The linear fit shown is used to convert the gauge pressure reading to actual pressure.

Compton and Biloiu also determined the pressure at $z=80 \mathrm{~cm}$ and $z=220 \mathrm{~cm}$ as a function of Balzer gauge readings with a typical pressure gradient present when the pumps are on (with no plasma discharge) by placing the Baratron gauge at each location. This was done by again introducing increasing amounts of argon gas into the chamber and recording readings of each gauge. See Appendix A for data and graphs of these measurements. The data acquisition code uses the following equations to determine the pressure at $z=80 \mathrm{~cm}$ given the front gauge pressure reading $P_{G}$ (unconverted):

$$
\begin{aligned}
& P=0.02+0.5 \times P_{G}, P_{G}<4 m \text { Torr } \\
& P=-0.3+0.8 \times P_{G}, P_{G}>4 m \text { Torr } .
\end{aligned}
$$

Similar measurements for helium, a gas not used in this work, are shown in Appendix A.

\subsection{Magnetic field}

Ten electromagnets produce a steady state axial magnetic field of 0-1300 Gauss in 
HELIX. Each magnet has 46 internal copper windings with a resistance of $17 \mathrm{~m} \Omega$ and an inductance of $1.2 \mathrm{mH}$. The magnets are water-cooled, and their axial positions are adjustable along a set of rails. Two Xantrex 200 Amp power supplies configured in parallel provide current to the electromagnets. These power supplies are different from those used previously ${ }^{1}$ to eliminate fluctuations that were discovered in the old MacroAmp power supply.

The LEIA magnetic field is created by a set of seven custom-built, 9' diameter electromagnets. Each magnet consists of five sets of aluminum tubing wound into two pancakes of four layers each, for a total of 40 turns. The 0.5 " $\mathrm{x} 0.5$ " tubing is hollow and wrapped in an insulating paper. The magnets are water-cooled by a closed system with a Neslab HX-300 chiller. These magnets are upgraded versions of those used in previous experiments. ${ }^{1}$ In these new electromagnets the larger size aluminum tubing and increased number of turns allow us to achieve a higher magnetic field strength. Additionally, the inner hole of the new tubing is circular, rather than rectangular, allowing for a much better attachment of the water connections. A magnetic field of 0-130 Gauss can be created using a 200 Amp DC EMHP power supply; an $~ 85 \%$ increase over the original magnets.

Upon completion of LEIA magnet building and installation of the new HELIX power supplies, several measurements of the axial (at $r=0$ ) magnetic field were made (shown in Figures 2.6-2.7). 


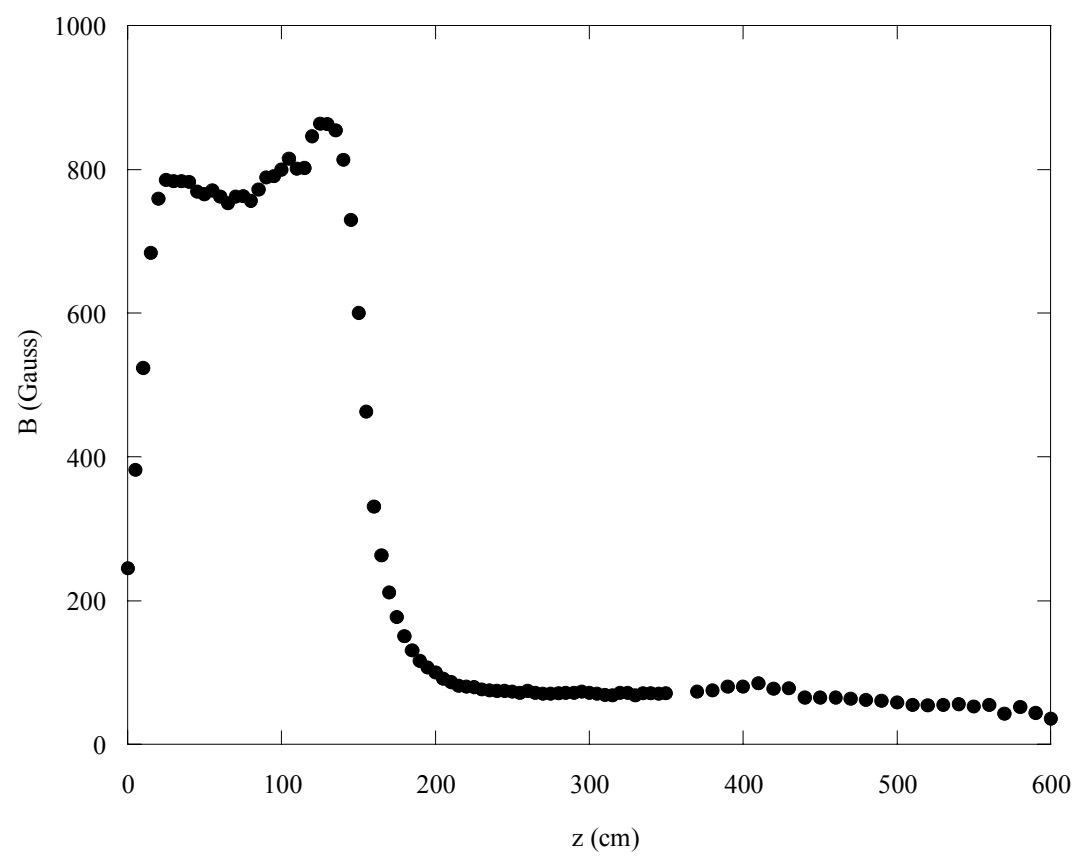

Figure 2.6 Axial profile of the magnetic field at $r=0$ for a current of 220 Amps in the HELIX magnets and $100 \mathrm{Amps}$ in the LEIA magnets. The axial distance is measured from the end of the HELIX Pyrex chamber and increases towards LEIA.

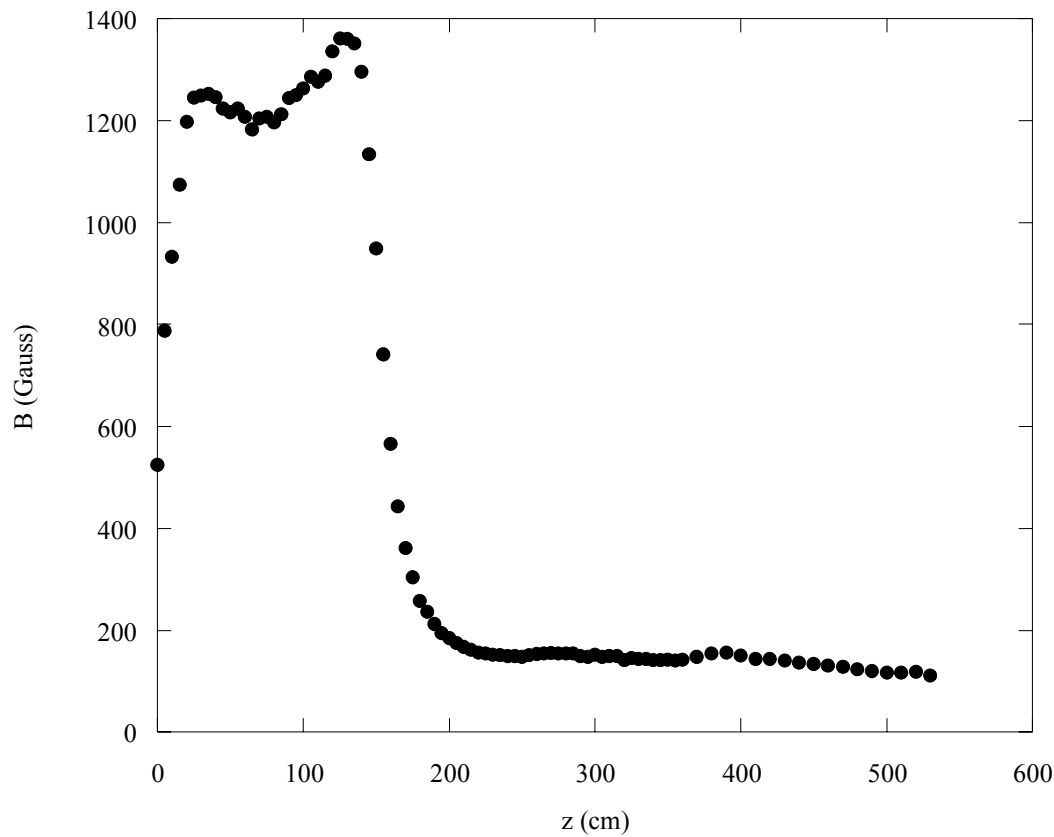

Figure 2.7 Axial profile of the magnetic field at $r=0$ for a current of 345 Amps in the HELIX magnets and $200 \mathrm{Amps}$ in the LEIA magnets. The axial distance is measured from the end of the HELIX Pyrex chamber and increases towards LEIA. 


\subsection{RF antenna and matching network}

To create a plasma in HELIX, RF power is coupled into a $19 \mathrm{~cm}$ half wave, righthanded helix antenna wrapped around the Pyrex chamber. An ENI $200030 \mathrm{~dB}$ amplifier provides up to $2 \mathrm{~kW}$ of power in a frequency range of 6-18 MHz, controlled by a $50 \mathrm{MHz}$ Wavetek function generator. For the experiments described here, the RF frequency was held at $9.5 \mathrm{MHz}$. The RF power is coupled to the antenna through a $\pi$-matching network that matches the inductive load of the antenna to the amplifier's output impedance of 50 Ohms. The matching network contains one load capacitor and three tuning capacitors, all of which are Jennings high voltage tunable vacuum capacitors. The load capacitor has a tunable range of 20-2000 pF, two of the tuning capacitors have a range of 4-250 pF, and the third tuning capacitor has a range of 5-500 pF. The capacitors are connected by sheets of copper, which are connected to the antenna by rods of silver-plated copper. A schematic of the matching circuit and connections to the antenna is shown in Figure 2.8.
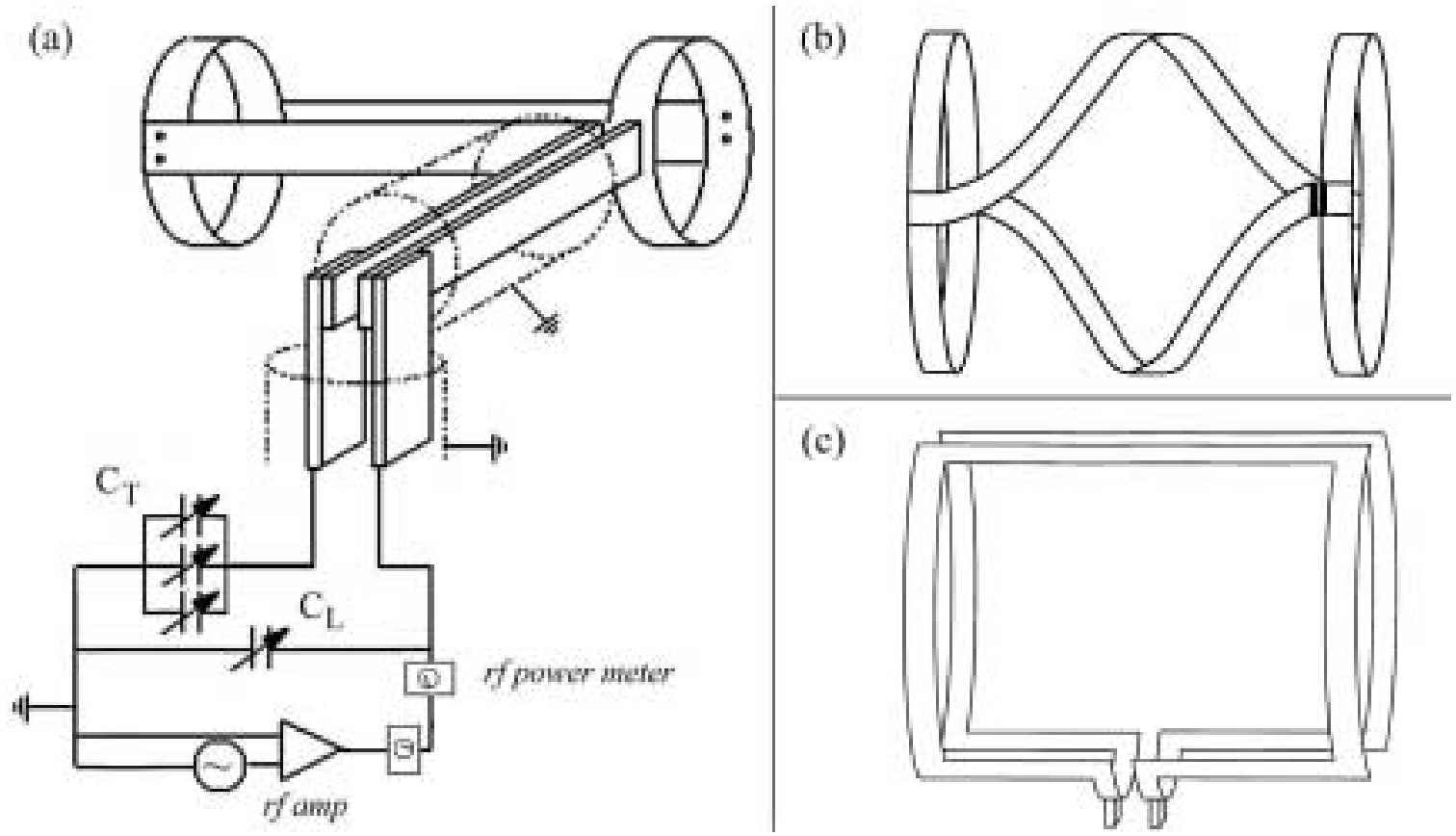

Figure 2.8 a) Antenna matching circuit for HELIX. ${ }^{2} \mathrm{C}_{\mathrm{T}}$ is the tuning capacitor and $\mathrm{C}_{\mathrm{L}}$ is the load capacitor. b) and c) show two additional RF antenna designs. Antenna b) is used in this work. 


\subsection{Plasma parameters}

The plasma can be operated in pulsed or steady state mode over a wide range of plasma parameters. For these experiments the plasma was typically operated steady state, at a RF frequency of $9.5 \mathrm{MHz}$, and a HELIX magnetic field of 750 Gauss. The LEIA magnetic field was set to zero because a non-zero field tended to cause the core, central bright plasma region, to be off-axis. The parameters that were varied include gas fill pressure and RF power. Additional typical parameters are shown in Table 2.1.

Table 2.1 Typical plasma parameters in HELIX.

\begin{tabular}{|l|l|}
\hline Plasma Parameter & Typical Value in HELIX \\
\hline Gas species & argon, helium \\
\hline Base pressure & $<2 \times 10-7$ mTorr \\
\hline Operating pressure & 0.1 to $100 \mathrm{mTorr}$ \\
\hline Magnetic field & $<1300$ Gauss \\
\hline RF power & 0 to $2 \mathrm{~kW}$ \\
\hline Operating frequency & $9.5 \mathrm{MHz}(6-18 \mathrm{MHz})$ \\
\hline Density & $\sim 10^{12} \mathrm{~cm}^{-3}$ \\
\hline Electron temperature & $\sim 5 \mathrm{eV}$ \\
\hline Ion temperature & $<1 \mathrm{eV}$ \\
\hline Electron gyroradius & $\sim 0.04 \mathrm{~mm}$ \\
\hline Ion gyroradius & $\sim 2.7 \mathrm{~mm}$ \\
\hline
\end{tabular}


For the collisional-radiative model, a measurement of the neutral pressure at the edge is needed. For the experiments reported in this work, I chose to use the measured fill pressure in the absence of the discharge obtained with the Baratron gauge located at position $A(z=80 \mathrm{~cm})$ in Figure 2.2. When the plasma discharge was turned on, the Balzers gauge reading increased while the Baratron gauge reading at $z=80 \mathrm{~cm}$ decreased. This may be an effect of RF noise, though Tynan has reported a similar decrease in the edge neutral pressure upon initiation of the discharge measured by a capacitance manometer. ${ }^{4} \mathrm{He}$ finds the same results occur whether the gauge is attached to the chamber wall or inserted into a radially scannable tube. Hanna and Watts also report a decrease in edge neutral pressure at discharge initiation using a thermocouple gauge. ${ }^{5}$ Table 2.2 shows a comparison of pressure gauge reading with and without discharge for various gas flow settings. As described by the table, with no plasma discharge, the Balzers gauge in the glass tee measures a higher pressure than does the Baratron located at the edge of the HELIX chamber. This is an expected pressure gradient due to the gas inlet located in the glass tee and the pumping configuration. Upon initiation of the plasma discharge, the Balzers pressure increases while the Baratron pressure decreases. This indicates that the actual pressure at the edge of the main plasma column is much lower than that upstream of the plasma column. Therefore, relying on pressures measured away from the plasma column could yield inaccurate results. A valve was used to separate the Baratron gauge from the plasma during operation to protect it from extended exposure to hot plasma. 
Table 2.2 Pressure gauge reading comparison.

\begin{tabular}{|l|l|l|l|l|}
\hline Flow $(\mathrm{sccm})$ & $\begin{array}{l}\text { HELIX Balzers } \\
\text { reading (mTorr) }\end{array}$ & $\begin{array}{l}\text { Balzers } \\
\text { conversion } \\
\text { (mTorr) }\end{array}$ & $\begin{array}{l}\text { Baratron } \\
\text { reading (mTorr) } \\
\mathrm{z}=80 \mathrm{~cm}\end{array}$ & $\begin{array}{l}\text { Discharge } \\
\text { status }\end{array}$ \\
\hline 80 & 5.4 & 8.6 & 4.1 & Off \\
\hline 80 & 5.7 & 9.1 & 3.8 & On \\
\hline 90 & 6.2 & 10.0 & 4.6 & Off \\
\hline 90 & 8.2 & 13.6 & 2.1 & On $(\mathrm{P}=350 \mathrm{~W})$ \\
\hline 90 & 9.7 & 16.3 & 0.3 & On $(\mathrm{P}=750 \mathrm{~W})$ \\
\hline 102 & 6.4 & 10.4 & 5.1 & Off \\
\hline 102 & 8.7 & 14.5 & 2.3 & On \\
\hline 114 & 7.3 & 12.0 & 6.0 & Off \\
\hline 114 & 10 & 16.9 & 3.2 & On \\
\hline
\end{tabular}




\section{References}

${ }^{1}$ J.L. Kline, Slow Wave Ion Heating and Parametric Instabilities in the HELIX Helicon Source, Ph.D. Dissertation, West Virginia University, Morgantown (2002).

${ }^{2}$ M.M. Balkey, Optimization of a Helicon Plasma Source for Maximum Density with Minimal Ion Heating, Ph.D. Dissertation, West Virginia University, Morgantown (2000).

${ }^{3}$ Balzers, Operating Manual for PKR 250 Compact Full Range Gauge, Balzers Aktiengesellschaft, FL9496 Balzers, Fürstentum Liechtenstein.

${ }^{4}$ G.R. Tynan, J. Appl. Phys. 86, 5356 (1999).

5 J. Hanna and C. Watts, Phys. Plasmas 8, 4251 (2001). 


\section{Chapter 3: Diagnostics}

\subsection{Laser-induced fluorescence}

Laser-induced fluorescence (LIF) is a widely-used method of measuring particle velocity distributions that has excellent spatial resolution and is non-perturbing. In 1975, Stern and Johnson were the first to perform LIF in plasma, using a single frequency argon ion laser. ${ }^{1}$ A tunable dye laser was first used for LIF in plasma in 1979 by Meng and Kunze, ${ }^{2}$ however Hill, Fornaca, and Wickham first used the ability to tune the laser for "velocity selective" LIF in $1983 .{ }^{3}$ Rather than measuring the emission line shape, which is limited by the resolution of the spectrometer used for detection, their method measures the absorption line shape such that velocity resolution is determined by the natural linewidth of the atomic transition. The dye laser was the laser of choice for LIF for many years due to its tunability, high output power, and useful wavelength range. Recently diode laser technology has improved yielding lasers in more wavelength ranges with increased power and tunability. Previous LIF measurements at WVU were primarily performed with a dye laser, the details of which can be found in Refs. [4 and 5]. We have taken advantage of the recent improvements in diode lasers, and all measurements reported here were performed with a single tunable diode laser.

\subsubsection{Diode laser}

“...the unique position held by the diode laser as a light source for physics research, its present role in atomic physics, and the potential for its future use...imply that the diode laser could have as significant an impact on 
modern physics as the personal computer."

$$
\text { - J.C. } \text { Camparo }^{6}
$$

The diode laser was actually invented before the dye laser; however the nonlinearity of intensity versus injection current, unstable spatial profile, requirement of liquid nitrogen temperatures, and widely spread cavity modes made them less desirable. ${ }^{6}$ As these problems have been overcome and diode lasers have become commercially available, they have been used in many scientific applications; particularly in atomic physics. Bölger and Diels conducted the first atomic physics experiment with a diode laser, observing photon echoes in a cesium vapor. ${ }^{7}$ Diode lasers currently available have several advantages over dye lasers: they are comparatively inexpensive and do not require high voltage power and water cooling, making them accessible to more research groups. They are capable of gigahertz modulation; they are smaller in size, allowing for better portability; and they are available from ultraviolet to infrared wavelengths.

A diode laser consists of several semiconductor layers. An injection current is sent through the active region between the $n$ - and $p$-type layers. The electrons and holes created by the current recombine and emit photons to produce the laser light. The laser wavelength region is determined by the band gap of the semiconductor. Lasers that emit in the region of $670 \mathrm{~nm}$ are made from an InGaAlP semiconductor. The semiconductors are doped to achieve the wavelength range desired. The optical path length of the cavity and the wavelength dependence of the gain curve both depend on temperature; thus laser tuning can be achieved by varying the temperature. The shifting of the gain curve as the temperature changes will cause jumps in wavelength from one longitudinal cavity mode to another. ${ }^{8}$ Figure 3.1 shows laser wavelength versus laser temperature for a Sacher 
Lasertechnik SAL-665-10 diode (the type of diode laser used in this work), in which the cavity mode jumps appear as large shifts in laser wavelength for small changes in temperature. The injection current also changes the wavelength, primarily by changing the laser temperature via Joule heating, but also by changing the index of refraction through changes in the charge carrier density. ${ }^{8}$ Since laser power also depends on laser current, injection current modification is not a practical means of wavelength tuning for our application. Varying the ambient pressure and applied magnetic field strength are alternative diode laser tuning methods. ${ }^{6}$

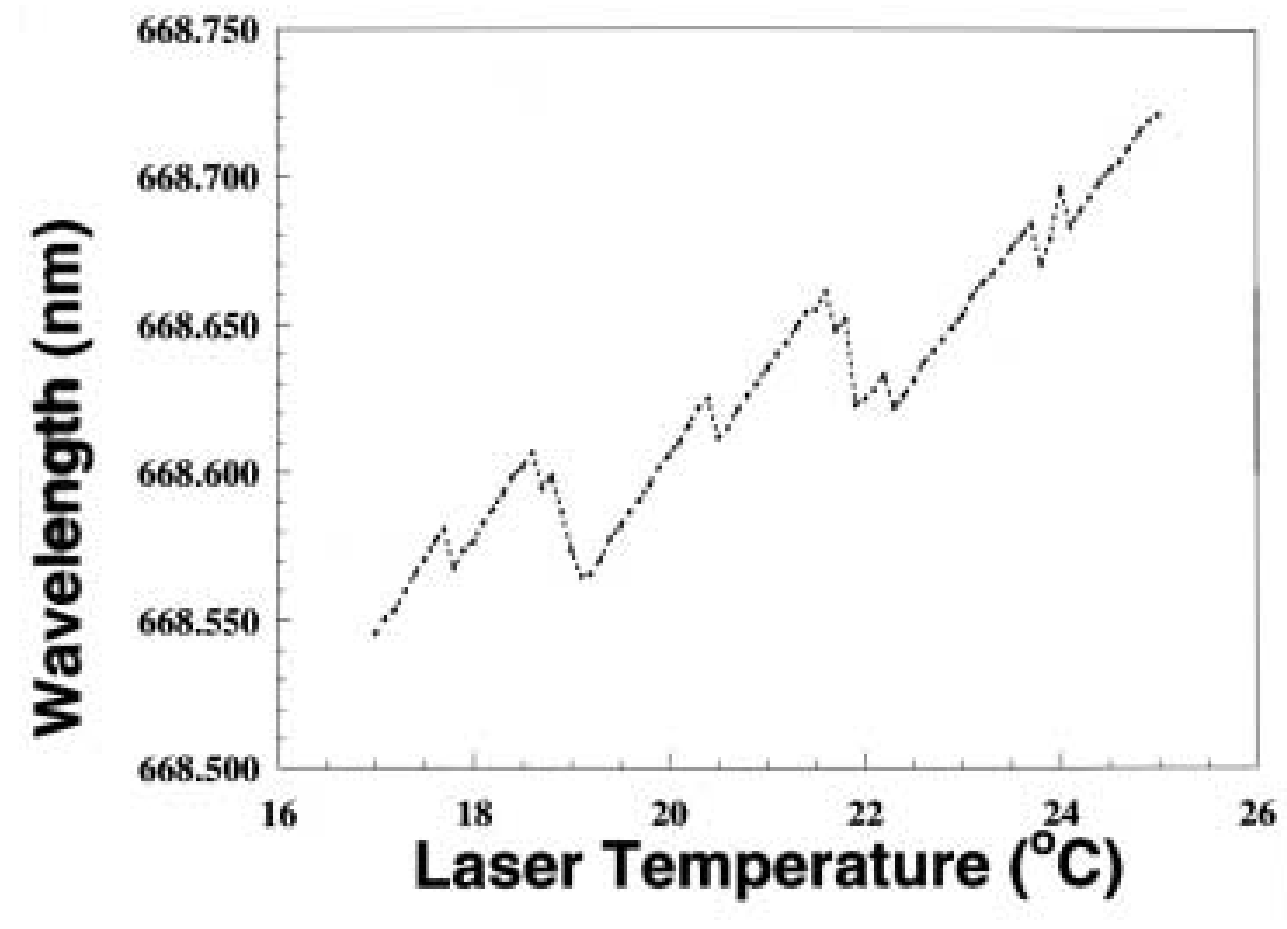

Figure 3.1 SAL-665-10 Laser wavelength versus temperature with laser piezo voltage at $50.0 \mathrm{~V}$.

Diode lasers can be configured in several ways to narrow the linewidth and control the central frequency. ${ }^{8}$ One option is to use an antireflection coating on the diode with external optics to create the laser resonator. This is known as an external cavity. A pseudo-external cavity setup has a diode with only one facet that has antireflection 
coating. Two types of pseudo-external cavity setups are the Littman/Metcalf $^{9}$ and Littrow $^{10}$ configurations. In the Littman/Metcalf configuration (shown in Figure 3.2), output from the diode is directed toward a grating at grazing incidence. The diffracted light reflects off the tuning mirror and back to the grating. Wavelength tuning is achieved by rotating the tuning mirror.

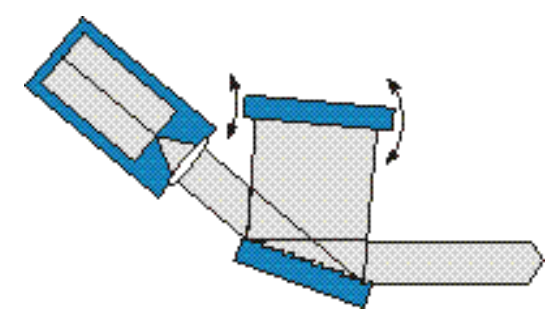

Figure 3.2 Littman/Metcalf configuration ${ }^{11}$

In the Littrow configuration, the grating is placed at the Littrow angle, defined such that the first-order diffracted beam is coincident with the input beam. The zeroth-order beam reflects off the grating and is directed out of the cavity. Wavelength tuning is achieved by changing the incidence angle of the beam upon the grating. The Littrow configuration is shown in Figure 3.3.

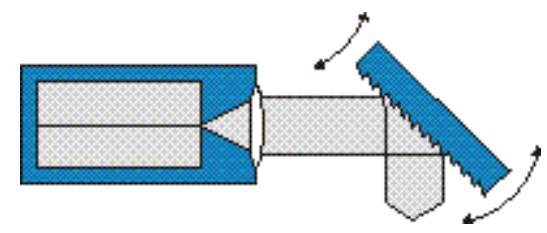

Figure 3.3 Littrow configuration ${ }^{11}$

The power output of the Littrow configuration is much greater than that of the Littman/Metcalf configuration because the zeroth-order beam is lost for Littman/Metcalf. However, the mode-hop-free tuning range in the Littrow configuration is smaller. Because laser power is critical to achieve significant LIF signal, the Littrow configuration 
was chosen for these experiments. ${ }^{12}$ The presence of a grating in these pseudo-external cavities enables wavelength tuning by moving the grating via piezoelectric control. Typical laser wavelength versus piezo voltage for the SAL-665-10 is shown in Figure 3.4 .

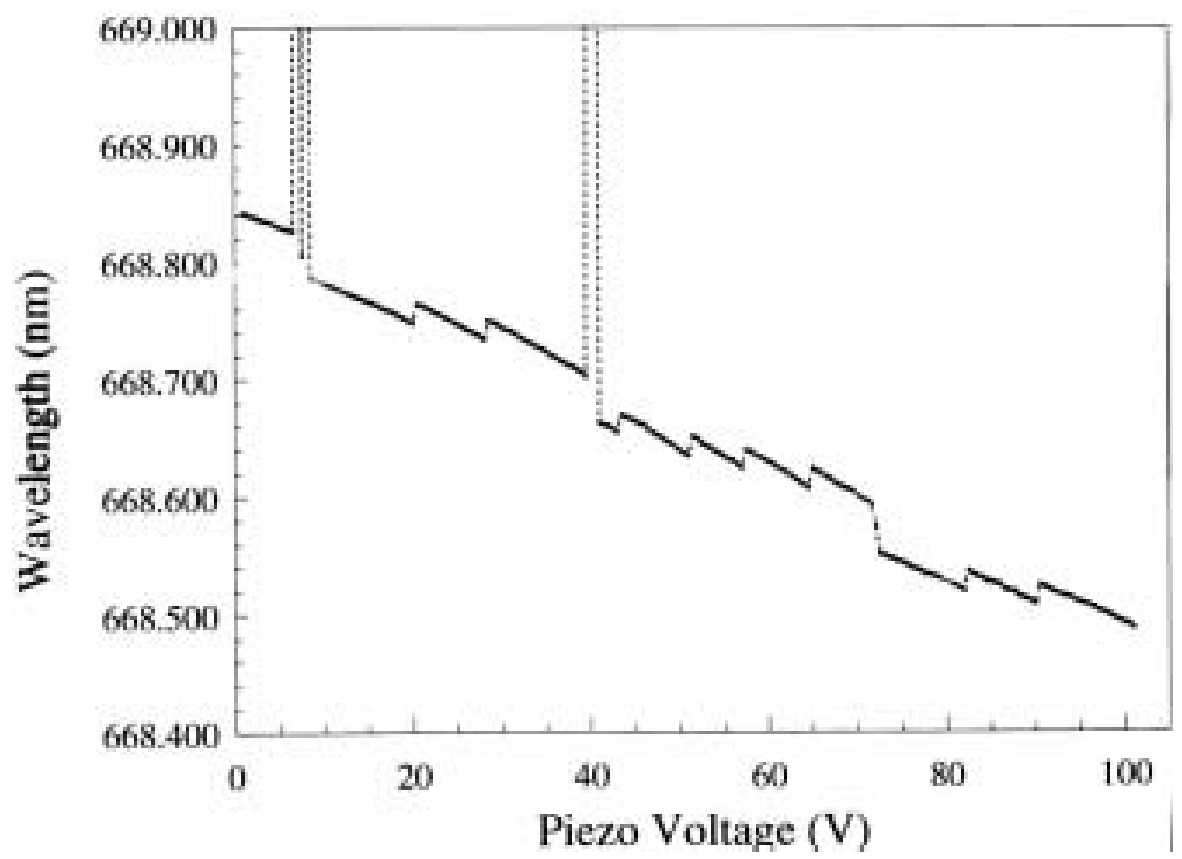

Figure 3.4 SAL-665-10 Laser wavelength versus piezo voltage with laser temperature at $21^{\circ} \mathrm{C}$.

\subsubsection{Diode laser based LIF diagnostic setup}

Our original $\leq 15 \mathrm{mWatt}, 1.5 \mathrm{MHz}$ bandwidth Sacher Lasertechnik SAL-665-10 diode laser was mounted in a Littrow external cavity and had a mode-hop free tuning range of up to $14 \mathrm{GHz}(\approx 0.021 \mathrm{~nm}){ }^{12}$ In April 2003, we upgraded our diode laser with another Littrow mounted diode, a SAL-665-20, with a mode-hop free tuning range of 12 $\mathrm{GHz}$ and an increased output power of $\leq 20 \mathrm{mWatt}$. In May 2005, an electromagnetic pulse (EMP) in the lab destroyed the diode, and we replaced it with a SAL-670-15, with a mode-hop free tuning range of $10 \mathrm{GHz}$ and an output power of $\leq 15 \mathrm{mWatt}$. The decrease 
in diode quality is due to the fact that the DVD industry began using diodes at a different wavelength $(685 \mathrm{~nm})$, making diodes at our desired wavelength less available. ${ }^{13}$ Most measurements described in this dissertation were performed with the SAL-670-15 diode. Characteristics of this diode are shown in Figure 3.5 through Figure 3.7. The mode hop characteristics of this diode were quite a bit different than our previous diodes (compare Figure 3.6 and Figure 3.7 to Figure 3.1 and Figure 3.4, respectively).

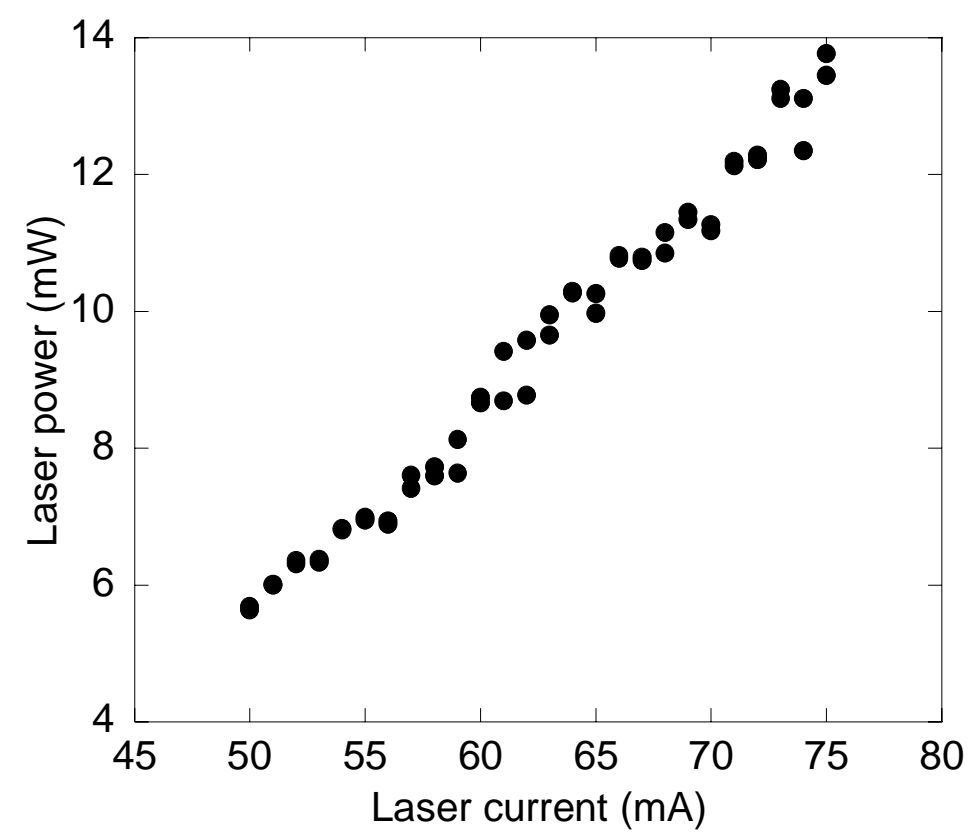

Figure 3.5 Laser power versus laser injection current for piezo voltage at $50.0 \mathrm{~V}$ and laser temperature at $21^{\circ} \mathrm{C}$ for the SAL-670-15 diode 


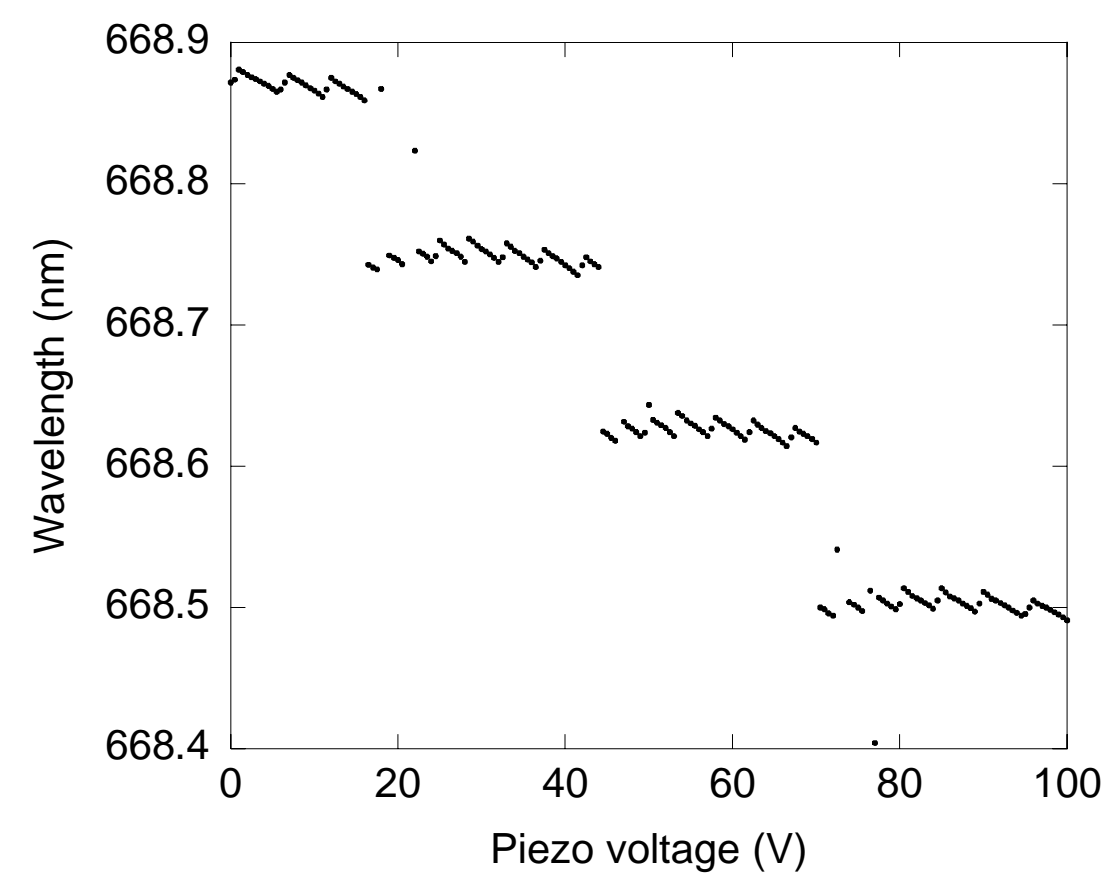

Figure 3.6 Laser wavelength versus piezo voltage for a laser temperature of $21{ }^{\circ} \mathrm{C}$

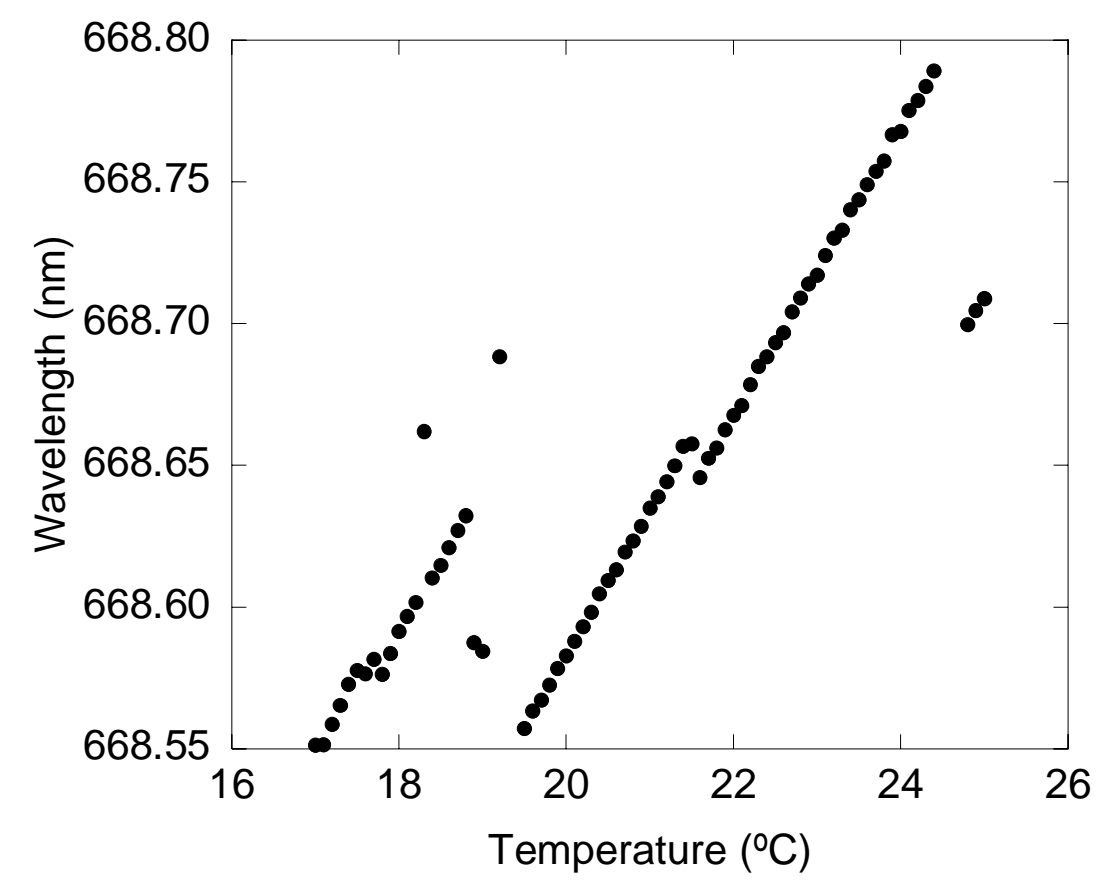

Figure 3.7 Laser wavelength versus laser temperature for a piezo voltage of $50.0 \mathrm{~V}$ 
The destruction of the laser diode by the EMP reflects the extreme sensitivity of these diode lasers to environmental conditions. Precautions that can be taken to reduce diode destruction include plugging the laser controller into an Uninterruptible Power Supply, turning the laser current on and off in gradual steps, and wearing a wrist grounding strap attached to the laser table. Special care should be taken when low humidity conditions could cause static buildup on the optical table or on personnel.

Frequency (wavelength) scanning is accomplished by varying the voltage on the piezoelectric-controlled grating located within the laser cavity. A National Instruments $\mathrm{I} / \mathrm{O}$ card provides the voltage ramp to scan the laser frequency. The custom LabWindows ${ }^{\mathrm{TM}}$ code used to create the voltage ramp includes a conversion factor to create a voltage ramp that provides the desired frequency range. This conversion factor is a unique characteristic of each diode used. For the SAL-670-15 used in these experiments, the conversion factor is $1.73 \mathrm{GHz} / \mathrm{V}$. The linearly polarized laser is mounted on a vibration-isolated platform to decrease drift in the laser frequency. The laser light is directed through an iodine cell, into a wavemeter, and into the plasma through a viewport at location $\mathrm{C}$ in Figure 2.2 with a series of mirrors and beam splitters mounted on the vacuum chamber and the laser table. 


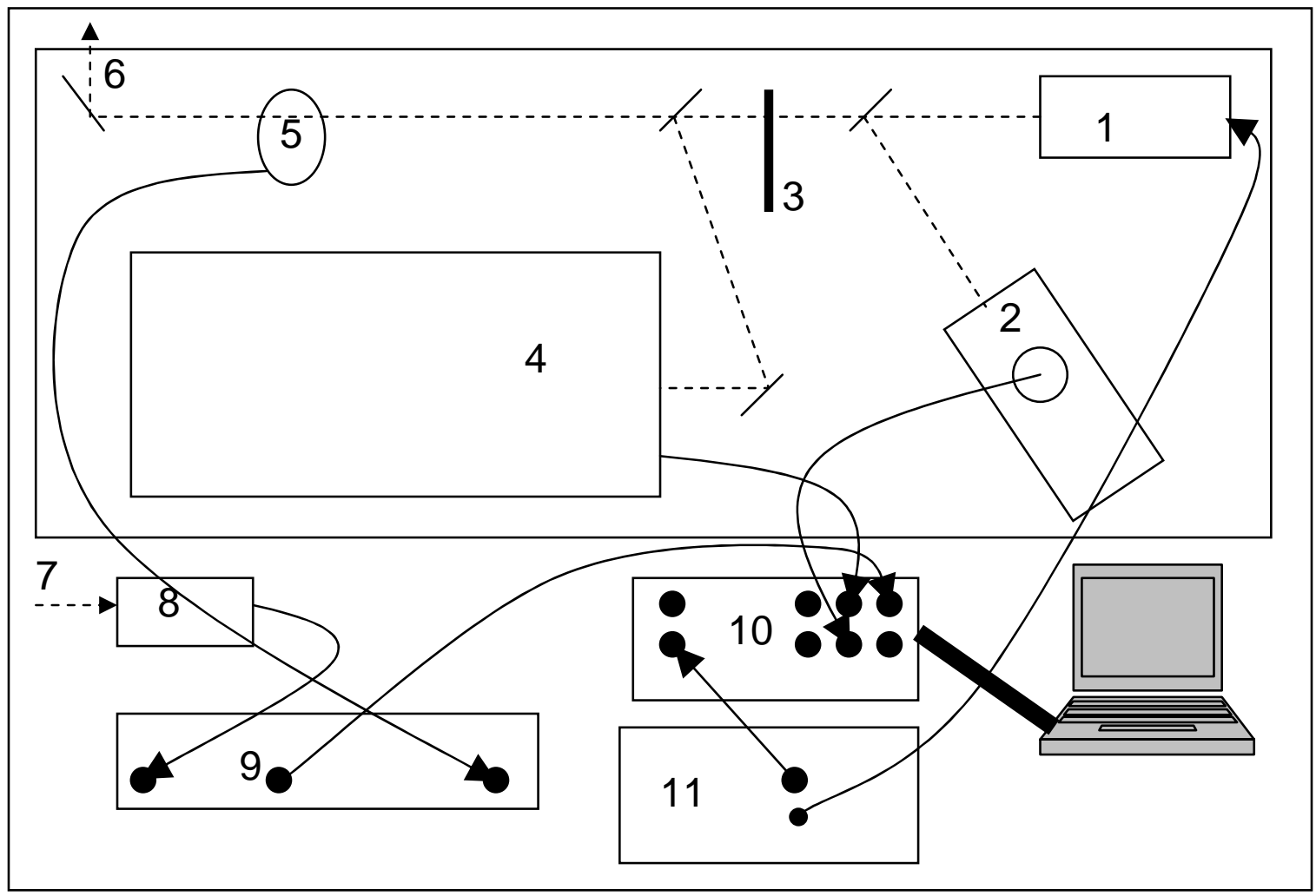

Figure 3.8 Diagnostic Configuration: 1. Laser head; 2. Iodine cell heater and photodiode detector; 3. Power meter; 4. Wavemeter; 5. Optical chopper; 6. Laser to plasma; 7. Light from collection optics in fiber; 8. Bandpass filter and PMT or infrared detector; 9. Lock-in amplifier; 10. I/O board; 11. Laser controller

A tightly focused set of optics, mounted on the horizontal arm of the scanning stage, collects the fluorescence light perpendicular to the injected beam and sends the light through a fiber optic cable to a filtered (1 $\mathrm{nm}$ wide bandpass) Hamamatsu photomultiplier tube (PMT) for argon ions or Hamamatsu infrared detector for argon neutrals. The PMT/detector signal is composed of fluorescence radiation, electron impact induced radiation and electronic noise. A mechanical chopper operating at a few $\mathrm{kHz}$ is used to modulate the laser beam before it enters the vacuum chamber, and a Stanford Research Systems SR830 lock-in amplifier is used to eliminate all non-correlated signals. Lock-in amplification is indispensable since the electron-impact induced emission is several orders of magnitude larger than the fluorescence signal. The laser wavelength is 
tuned to the appropriate transition with a Burleigh WA-1500 wavemeter and monitored for frequency drift with a simultaneous molecular iodine fluorescence measurement or wavemeter output during each scan of the laser frequency. The diagnostic configuration is shown in detail in Figure 3.8.

The three level LIF scheme for Ar II $^{14}$ consists of laser emission at $668.6139 \mathrm{~nm}$ (in vacuum) to pump the $3 \mathrm{~d}^{4} \mathrm{~F}_{7 / 2}$ state to the $4 \mathrm{p}^{4} \mathrm{D}^{0}{ }_{5 / 2}$ state, which then decays to the $4 \mathrm{~s}$ ${ }^{4} \mathrm{P}_{3 / 2}$ state, emitting a photon at $442.7244 \mathrm{~nm}$. As is the case for most LIF schemes, the pump state is a metastable state, ensuring a large population from which to excite the ions.

Our three level LIF scheme for Ar I (see Figure 3.9) uses laser emission at $667.9126 \mathrm{~nm}$ (in vacuum) to pump the $4 \mathrm{~s}[3 / 2]^{\circ}{ }_{1}$ state to the $4 \mathrm{p}^{\prime}[1 / 2]_{0}$ state, which then decays to the $4 \mathrm{~s}^{\prime}[1 / 2]^{\circ}{ }_{1}$ state, emitting a photon at $750.5934 \mathrm{~nm}$. While the $4 \mathrm{~s}[3 / 2]^{\circ}{ }_{1}$ state is not a ground or metastable state, we expected a sufficient population for LIF due to direct excitation from the ground state and electron-impact excitation transfers from nearby metastable states $4 \mathrm{~s}[3 / 2]^{\circ}$ and $4 \mathrm{~s}^{\prime}[1 / 2]^{\circ}{ }_{0}$. 


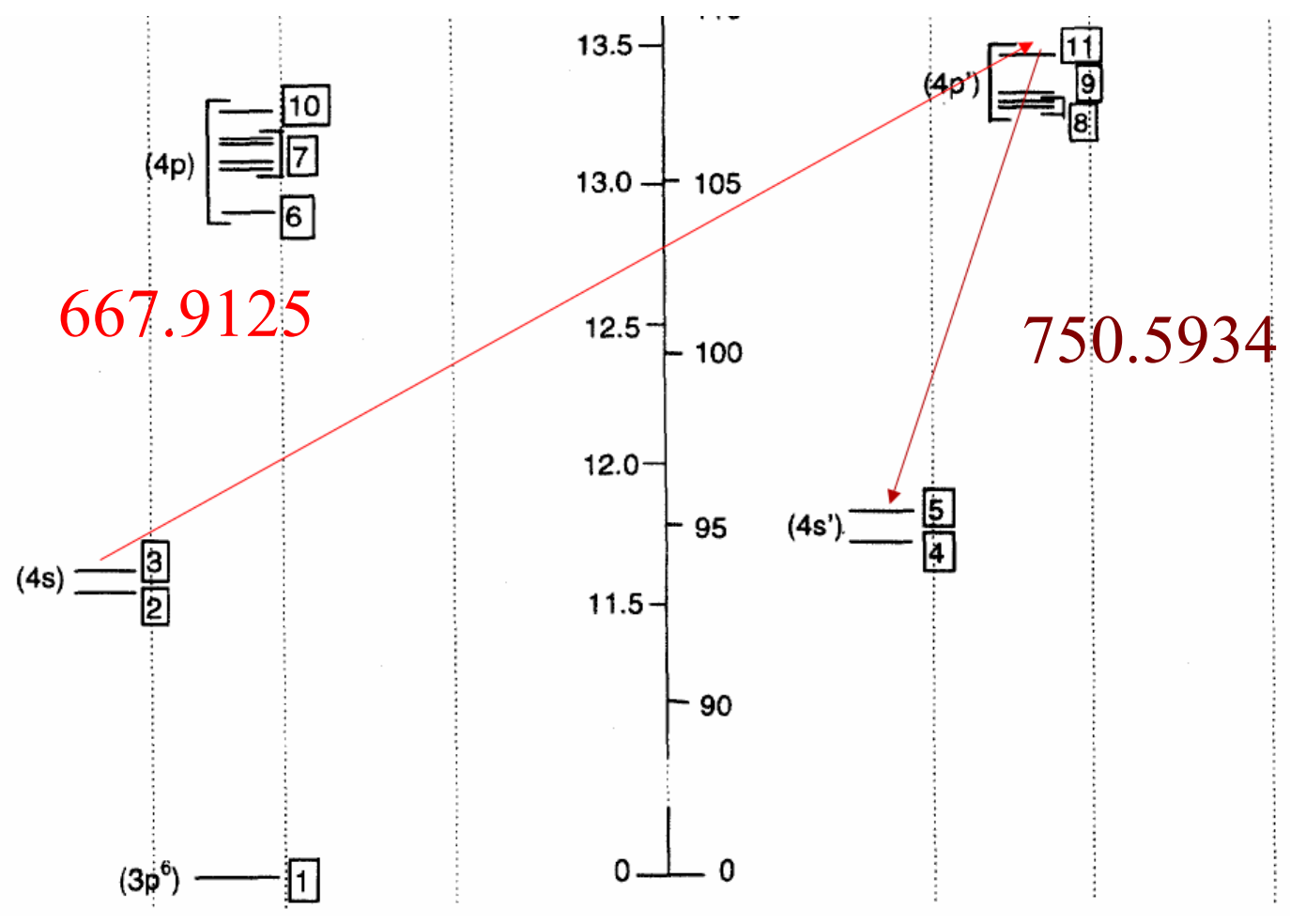

Figure 3.9 Diagram of Ar I LIF scheme.

The spectral resolution of the LIF diagnostic will be the larger of the laser linewidth and the natural linewidth of the absorption transition. The diode laser has a linewidth on the order of a megahertz. ${ }^{15}$ The natural linewidth of a transition from level $i$ with energy $E_{i}$ to level $k$ with energy $E_{k}$ is caused by the uncertainty

$$
\Delta E_{j}=\frac{h}{2 \pi \tau_{j}}
$$

in the energy of each level, where $\tau_{j}$ is the lifetime of level $j$, and $h$ is Planck's constant. The lifetime of level $j$ is given by

$$
\tau_{j}=\frac{1}{\sum_{l<j} A_{j l}},
$$

the sum of the Einstein transition probabilities $A_{j l}$ for spontaneous transitions from level $j$ to level $l$. Because our measurements are typically in frequency space, we would like to 
know the linewidth in terms of frequency, $\Delta v$. Using $E=h v$ and Equation (3.2), Equation (3.1) becomes

$$
\Delta v_{i j}=\frac{1}{2 \pi}\left(\sum_{m<i} A_{i m}+\sum_{n<j} A_{j n}\right) .
$$

For the Ar II LIF scheme, the linewidth of the $668.6139 \mathrm{~nm}$ transition from the $3 \mathrm{~d}$ ${ }^{4} \mathrm{~F}_{7 / 2}$ state to the $4 \mathrm{p}^{4} \mathrm{D}^{0}{ }_{5 / 2}$ state will only depend on the transition probabilities of the transitions out of the upper state, because the lower state is metastable, such that the transition probabilities out of that state are negligible. The transitions out of the upper state and the respective transition probabilities are given in Table $3.1 .^{16}$ Using these values and Equation (3.3), the linewidth is $\Delta v=2.13 \mathrm{MHz}$. Since this is larger than the laser linewidth, the natural linewidth limits the spectral resolution of the LIF diagnostic.

Table 3.1 Transition probabilities for calculating the linewidth of the Ar II LIF scheme absorption transition.

\begin{tabular}{|l|l|l|}
\hline Upper State & Lower State & $\mathrm{A}\left(10^{8} \mathrm{~s}^{-1}\right)$ \\
\hline $4 \mathrm{p}^{4} \mathrm{D}^{0}{ }_{5 / 2}$ & $3 \mathrm{~d}^{4} \mathrm{~F}_{3 / 2}$ & 0.002 \\
\hline $4 \mathrm{p}^{4} \mathrm{D}_{5 / 2}^{0}$ & $3 \mathrm{~d}^{4} \mathrm{~F}_{5 / 2}$ & 0.025 \\
\hline $4 \mathrm{p}^{4} \mathrm{D}^{0}{ }_{5 / 2}$ & $3 \mathrm{~d}^{4} \mathrm{~F}_{7 / 2}$ & 0.107 \\
\hline
\end{tabular}

For the Ar I LIF scheme, the linewidth of the $667.9126 \mathrm{~nm}$ transition from the $4 \mathrm{~s}[3 / 2]^{\circ}{ }_{1}$ state to the $4 p^{\prime}[1 / 2]_{0}$ state will depend on transitions out of both states since the lower state is not metastable. The transitions out of these states and the respective transition probabilities are given in Table 3.2. ${ }^{17}$ Using these values and Equation (3.3), the linewidth is $\Delta v=26.5 \mathrm{MHz}$. Since this is much larger than the laser linewidth, the natural linewidth also limits the spectral resolution of the Ar I LIF measurement. 
Table 3.2 Transition probabilities for calculating the linewidth of the Ar I LIF scheme absorption transition.

\begin{tabular}{|l|l|l|}
\hline Upper State & Lower State & $\mathrm{A}\left(10^{8} \mathrm{~s}^{-1}\right)$ \\
\hline $4 \mathrm{~s}[3 / 2]^{\circ}{ }_{1}$ & ${ }^{\mathrm{I}} \mathrm{S}_{0}$ (ground) & 1.19 \\
\hline $4 \mathrm{p},[1 / 2]_{0}$ & $4 \mathrm{~s}[3 / 2]^{\circ}{ }_{1}$ & 0.00241 \\
\hline $4 \mathrm{p},[1 / 2]_{0}$ & $4 \mathrm{~s}^{\prime}[1 / 2]^{\circ}{ }_{1}$ & 0.472 \\
\hline
\end{tabular}

To obtain reasonable Ar I LIF signal-to-noise, the source was operated at pressures above $10.5 \mathrm{mTorr}$ (operating pressure). Optimal signal-noise was achieved for neutral pressures of approximately $17 \mathrm{mTorr}$ (operating pressure). These relatively high neutral pressures provide the collision rates necessary to populate the initial $4 \mathrm{~s}[3 / 2]^{\circ}{ }_{1}$ state. A typical Ar I LIF measurement is shown in Figure 3.10.

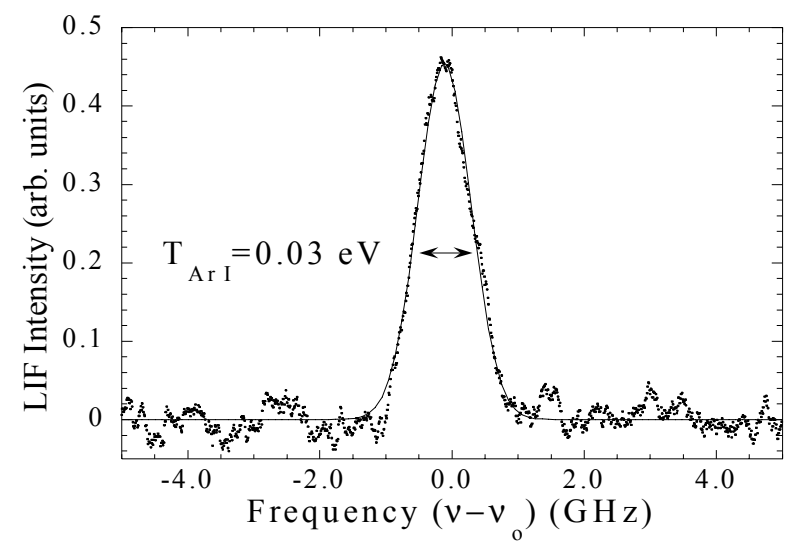

Figure 3.10 Typical Ar I LIF signal versus laser frequency and Maxwellian fit to the distribution (solid line).

The LIF signal for a three-level LIF scheme is directly proportional to the density of the initial state $(i)$ in the collection volume: ${ }^{18}$ 


$$
I \propto \frac{d \Omega}{4 \pi} A_{m, f} \int d^{3} x \int d^{3} v N_{i}(x, v) \frac{B_{m, i}}{4 \pi} \int_{0}^{\infty} d v L_{m, i}(v, v) W(x, v),
$$

where $d \Omega$ is the detector's solid angle, $A_{m, f}$ is the Einstein transition coefficient from the middle $(m)$ to final $(f)$ state (fluorescence transition), $N_{i}$ is the phase space density of the initial state, $B_{m, i}$ is the Einstein absorption coefficient from the initial to middle state, $L_{m, i}$ is the absorption line shape of the initial to middle state transition, and $W$ is the laser intensity line shape. As mentioned above, the laser linewidth is on the order of a megahertz. For typical helicon source argon plasma parameters, the total absorption lineshape is a convolution of thermal (Doppler) broadening and Zeeman splitting. Because the Doppler width and Zeeman splittings are on the order of a gigahertz, other effects such as the natural linewidth of the line and Stark broadening are ignorable. ${ }^{19}$

Thus, the total absorption lineshape consists of a number of Zeeman split components that are Doppler broadened. These components include $\pi$ transitions $(\Delta M=$ $0 ; M$ is the magnetic orbital quantum number) and $\sigma$ transitions (half for $\Delta M=+1$, half for $\Delta M=-1)$. The relative intensities of the Zeeman components obey $I_{\pi}=2 I_{\sigma}$. When the polarization axis of the laser is oriented parallel to the axial magnetic field (laser injection perpendicular to the chamber axis), only the $\pi$ transition is pumped. For parallel laser injection, the $\sigma$ transitions are pumped. Insertion of a quarter-wavelength retarder in the laser path creates circularly polarized light and enables pumping of either the $\Delta M=$ +1 or $\Delta M=-1$ transitions for parallel laser injection. Each Zeeman component of the measured LIF intensity can be integrated over the essentially delta function laser lineshape to yield a LIF intensity that is proportional to the bulk flow-shifted Maxwellian distribution of the interrogated species: ${ }^{20}$ 


$$
I(v)=I\left(v_{0}\right) \exp \left[\frac{-\left(v-\Delta v_{Z}-v_{0}-\mathrm{V}_{\mathrm{o}} v_{0} / c\right)^{2}}{\alpha_{D} T}\right]
$$

where $I(v)$ is the measured LIF signal, $\Delta v_{Z}$ is the Zeeman shift relative to the rest frame transition frequency $v_{o}\left(\Delta v_{Z}=\varepsilon B ; \varepsilon\right.$ is the shift coefficient for the Zeeman component; and $B$ is the magnetic field in $\mathrm{kG}),\left(\mathrm{V}_{\mathrm{o}} v_{o} / c\right)$ is the overall Doppler shift of the distribution due to bulk flow of the species, $\alpha_{D}$ scales the width of the thermal broadening for the argon species of mass $m\left(\alpha_{D}=2 k v_{0}^{2} / m c^{2}\right)$, and $T$ is the temperature of the species in $\mathrm{eV}$. The total measured LIF intensity is a sum of Equation (3.5) for all Zeeman components.

For the Ar II LIF scheme, the transition consists of eighteen Zeeman split components. This includes six $\pi$ transitions and $12 \sigma$ transitions. The Zeeman broadening of the $\pi$ lines due to a 1 kGauss magnetic field is $9.8 \times 10^{-4} \mathrm{~nm}\left(\Delta v_{Z} \sim 0.7 \mathrm{GHz}\right)$. The Zeeman shift of each set of $\sigma$ lines due to a $1 \mathrm{kGauss}$ magnetic field is approximately \pm $2.22 \times 10^{-3} \mathrm{~nm}(\sim 1.5 \mathrm{GHz})$ from the central wavelength, while the Zeeman broadening of each set is approximately $9.0 \times 10^{-4} \mathrm{~nm}\left(\Delta v_{Z} \sim 0.6 \mathrm{GHz}\right){ }^{20}$

For the specific Ar I transition used, the Zeeman splitting yields three components. $^{21}$ There is a single $\pi$ transition which is unshifted $(\varepsilon=0)$ from the central wavelength. For the two circularly polarized $\sigma$ transitions, the shift coefficients are $\varepsilon= \pm$ $1.4 \mathrm{GHz}$. Thus, the line shifts in a 1 kGauss magnetic field are $\Delta \lambda= \pm 2.08 \times 10^{-3} \mathrm{~nm}^{22}$ Because only a single transition is pumped for both parallel and perpendicular laser injection (when a quarter waveplate is used), all the Ar I LIF measurements are of the form of Equation (3.5).

A fit of Eq. (3.5) to the Ar I $\pi$ transition LIF measurement shown in Figure 3.10 yields a neutral temperature of $0.03 \mathrm{eV}$. Given the relatively high neutral pressures 
required for the LIF measurement, a neutral temperature consistent with temperature of the chamber walls, $\sim 0.03 \mathrm{eV}$, is not surprising. The relative density of the initial state is obtained by integrating the LIF measurement over laser frequency.

One issue that has to be considered with any laser-based plasma diagnostic is saturation. Saturation occurs when the laser intensity is high enough to pump most of the ions or neutrals out of the initial state. The remaining ions or neutrals in the initial state cannot absorb all the incident laser light and the LIF signal plateaus, rather than increasing linearly with laser power. Figure 3.11 shows LIF signal versus laser power for the Ar II LIF scheme at $x=0 \mathrm{~cm}$. The total LIF signal is given by the area under the LIF curve, proportional to the product of the amplitude and square root of the temperature from the Maxwellian fit to the LIF data.

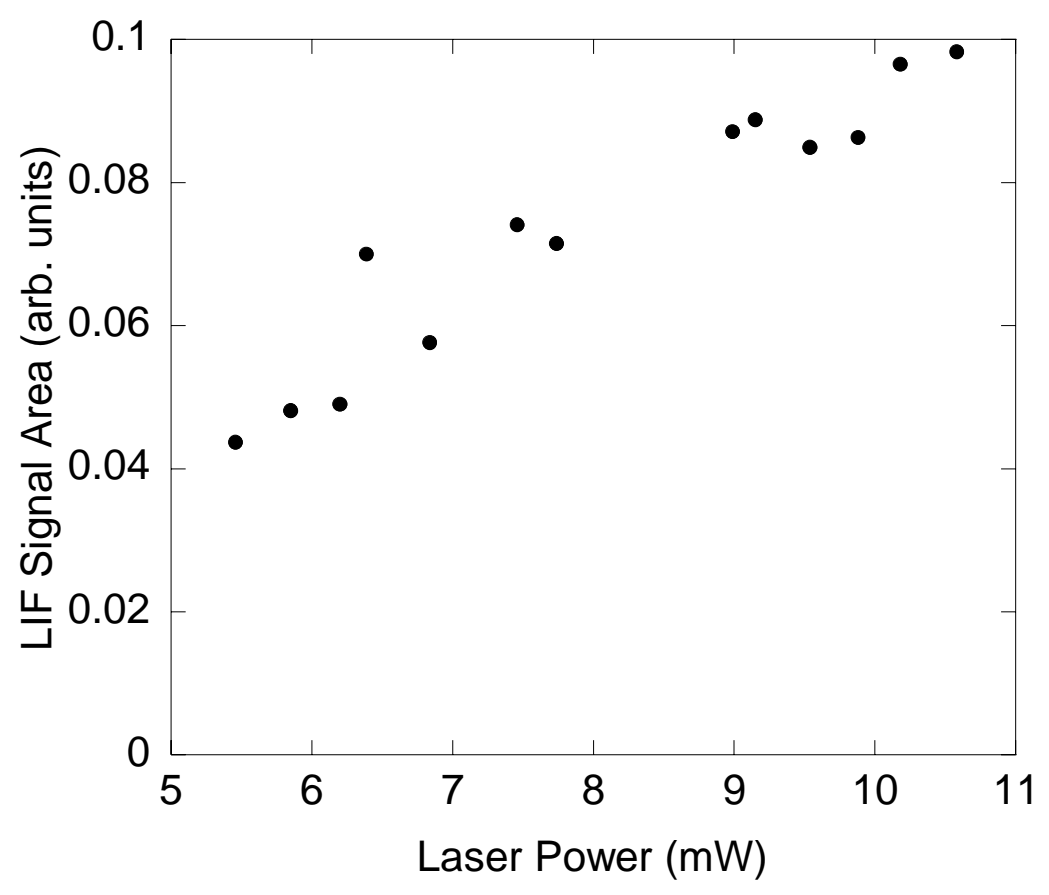

Figure 3.11 LIF signal versus laser power for Ar II LIF at $x=0 \mathrm{~cm}$ 
For this transition, the LIF signal is linear with laser power up the highest diode laser powers available. Thus, laser powers up to $11 \mathrm{~mW}$ do not saturate the Ar II pump state. A similar plot for the Ar II LIF scheme at $x=4 \mathrm{~cm}$, near the edge of the plasma, is shown in Figure 3.12.

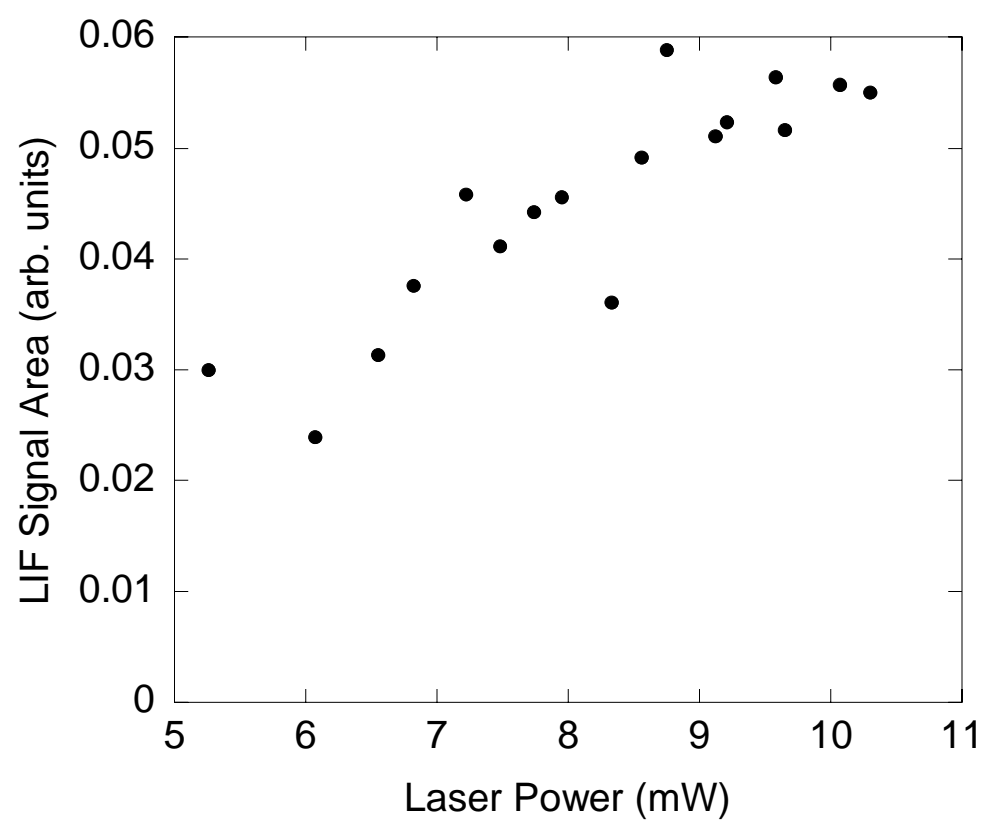

Figure 3.12 LIF signal versus laser power for Ar II LIF at $x=4 \mathrm{~cm}$

To determine if the initial state for the Ar I scheme is saturated, similar experiments were performed. The results are shown in Figure 3.13 for $x=0$ and in Figure 3.14 for $x=3 \mathrm{~cm}$. These measurements indicate a linear dependence of LIF signal on laser power (albeit with a much smaller slope than for the Ar II scheme), confirming that the initial state is not saturated for laser powers up to $11 \mathrm{~mW}$. 


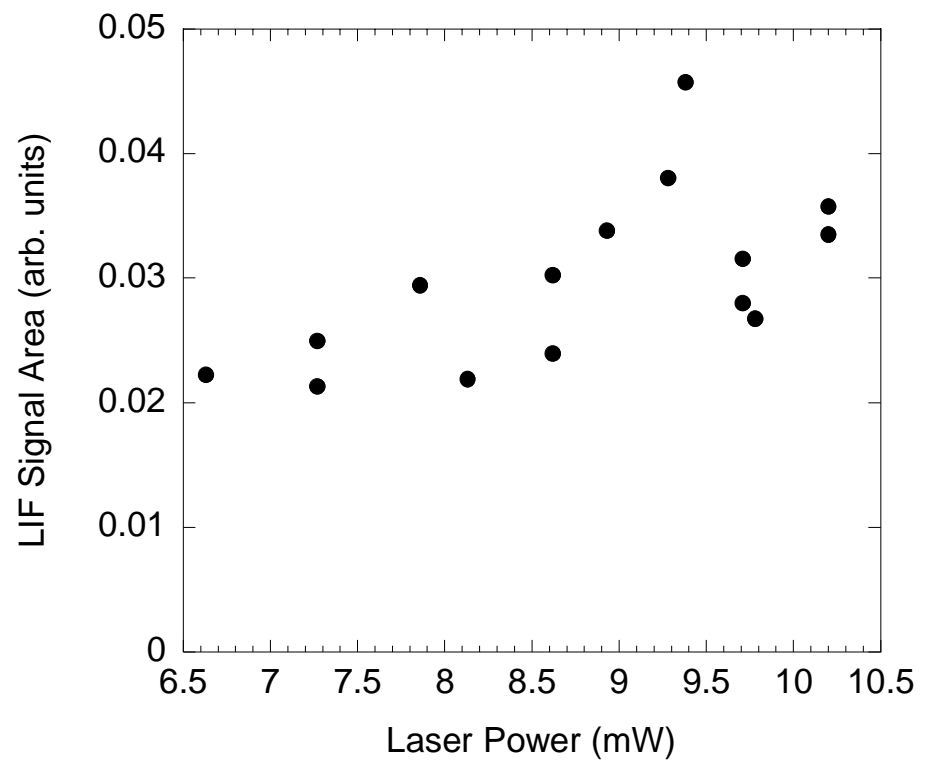

Figure 3.13 LIF signal versus laser power for Ar I LIF at $x=0 \mathrm{~cm}$

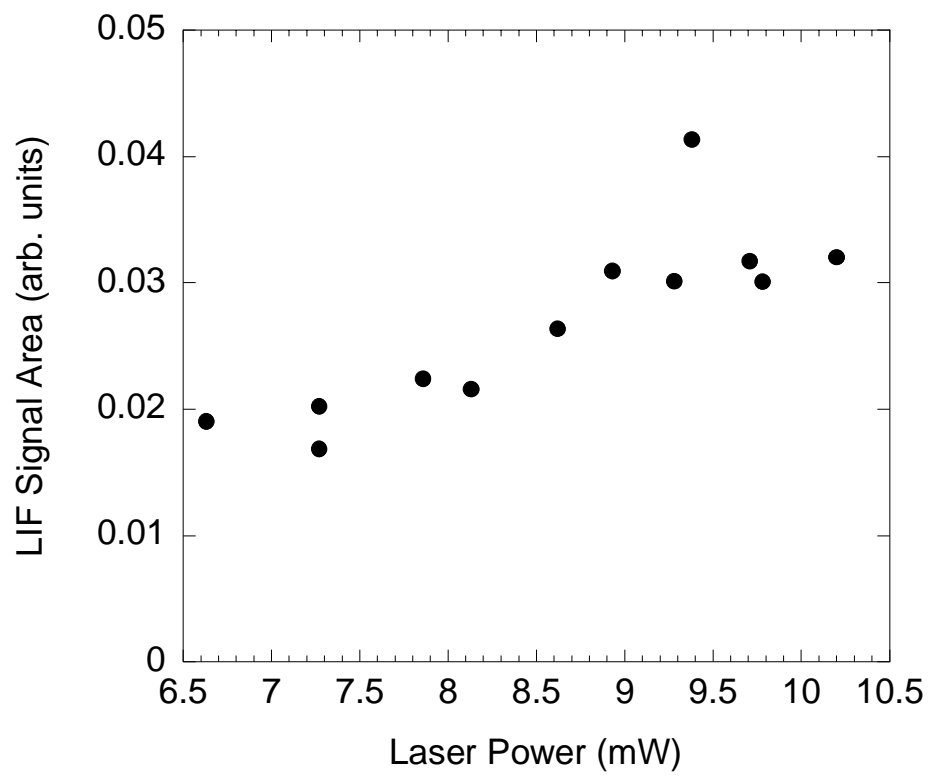

Figure 3.14 LIF signal versus laser power for Ar I LIF at $x=3 \mathrm{~cm}$ 
When measuring across the diameter of the plasma by scanning the collection optics, the incident light on the PMT can vary with radius, generally increasing toward the center. For optimal signal levels, the PMT voltage can be adjusted during the radial scan. Because the LIF measurements are not automatically normalized with respect to PMT voltage, the data must be corrected by hand after the radial scan is performed. Each time the PMT voltage required adjustment, a measurement was taken at the same radial position with both the old and new PMT voltage level. A conversion factor for the PMT voltage change was calculated by dividing the area of the curve measured at higher PMT voltage by the area of the curve measured at lower PMT voltage. All measurements across the diameter of the plasma were then normalized to a single PMT voltage using these conversion factors. For the complete radial profiles presented in this work, the PMT voltage was constant across the plasma diameter, eliminating the need for this calibration.

\subsubsection{Iodine cell}

The shift of the measured velocity distribution from the LIF absorption frequency (wavelength) is used to determine the bulk flow of the interrogated species for all three LIF schemes. A particle, atom or ion, moving towards the laser will see the frequency higher than that of the actual frequency. Thus, the laser will excite the particle at a lower laser frequency (higher wavelength) than if the particle were at rest. Measurements of fluorescence spectrum from an iodine cell during the frequency scan of the laser provide an absolute measurement of laser wavelength. Thus, the absolute shift in the absorption line is measured during each LIF measurement.

Because the weak absorption lines of molecular iodine in the wavelength range of all three LIF schemes are difficult to fluoresce in commercially available iodine cells, a 
modified iodine cell is required for use with our low power diode laser. Increased vapor pressure of molecular iodine in the reference cell can be obtained with either a custom iodine cell containing a larger than normal quantity of crystalline iodine, or by heating a commercially available iodine cell. We chose to construct a thermostatically controlled heater for our $100 \mathrm{~mm}$ long, $25 \mathrm{~mm}$ diameter Opthos Instruments iodine cell. Watlow flexible silicone rubber heaters were attached to the cell and the heated cell placed in a thick walled, aluminum housing (see Figure 3.15). The Watlow heaters are controlled by an Omega CNi3252 process controller using a K-type thermocouple for temperature monitoring. Oriel iris diaphragms were mounted on each end of the housing to limit ambient light while allowing the laser beam to pass through the cell. Fluorescent emission from the molecular iodine vapor is detected with a battery powered, UDT Sensors PIN photodiode and amplified with a Stanford Research Systems SR560 Preamplifier. For an incident laser power of $17 \mathrm{~mW}$, excellent signal to noise for the molecular iodine fluorescence measurement was obtained for an iodine cell temperature of $69^{\circ} \mathrm{C}$.

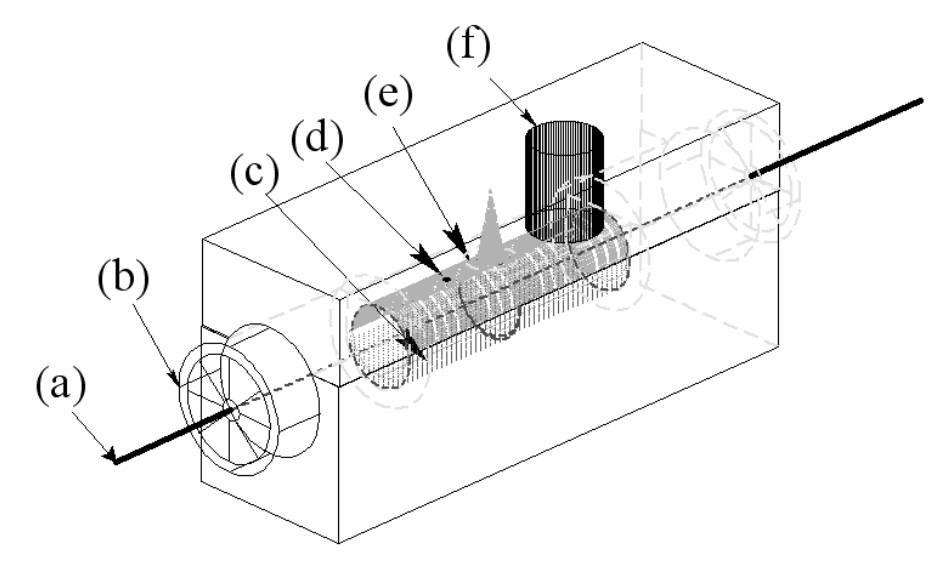

Figure 3.15 Housing for the heated iodine cell: (a) laser beam, (b) iris diaphragm, (c) flexible silicone heaters, (d) thermocouple, (e) iodine cell, and (f) photodiode detector. 
To calculate the absolute frequency shift, the reference iodine cell fluorescence peaks need to be known accurately. The absolute wavelengths and relative intensities of the molecular iodine fluorescence for a $12 \mathrm{GHz}$-wide scan in the vicinity of the central absorption wavelength of each of the three different LIF schemes are shown in Figure 3.16. (See Appendix B for raw data.)
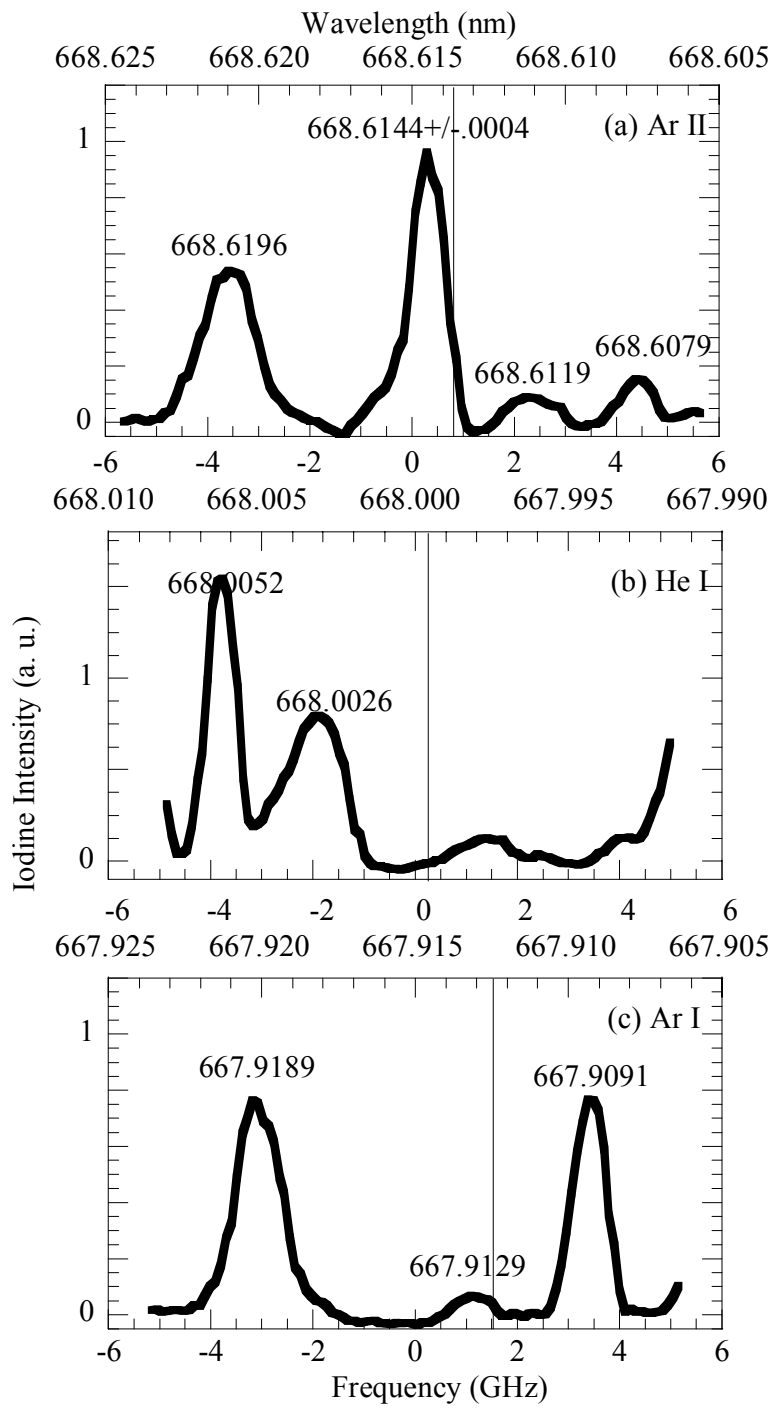

Figure 3.16 Intensity of iodine fluorescence signal versus frequency shift (and wavelength) around the lines used for (a) Ar II, (b) He I, and (c) Ar I LIF with the diode laser. The location of the pump line is indicated by a vertical line. 
The absolute wavelengths of each fluorescence peak were determined with the Burleigh wavemeter (with an absolute accuracy of $\pm .00003 \mathrm{~nm}$ according to the manufacturer and an observed statistical uncertainty of $\pm .0004 \mathrm{~nm}$ ). The temperature of the heated iodine cell was kept at $69.3 \pm 0.4{ }^{\circ} \mathrm{C}$. None of these very weak iodine peaks (all of Figure 3.16) appear in the standard iodine tables. The spacing in GHz between the LIF peak and the nearest iodine cell peak is $-0.40,-2.02$, and +2.28 for $\mathrm{Ar}$ II, He I, and $\mathrm{Ar}$ I, respectively. Figure 3.17 shows a $120 \mathrm{GHz}$-wide scan of the iodine fluorescence spectrum for the Ar II sequence with the Ar II pump line indicated by a vertical line at zero GHz. As in Figure 3 of Ref. [14], the location of molecular iodine absorption lines in the published atlas ${ }^{23}$ are indicated by solid circles. Note that most of the molecular iodine fluorescence lines do not appear in the standard atlas of absorption lines. This discrepancy is not restricted to only weak lines as a number of the fluorescence lines that are missing from the absorption atlas are "stronger" than those that do correspond to absorption lines in the atlas. A possible explanation for this could be that additional vibrational modes are excited by heating the iodine cell. 


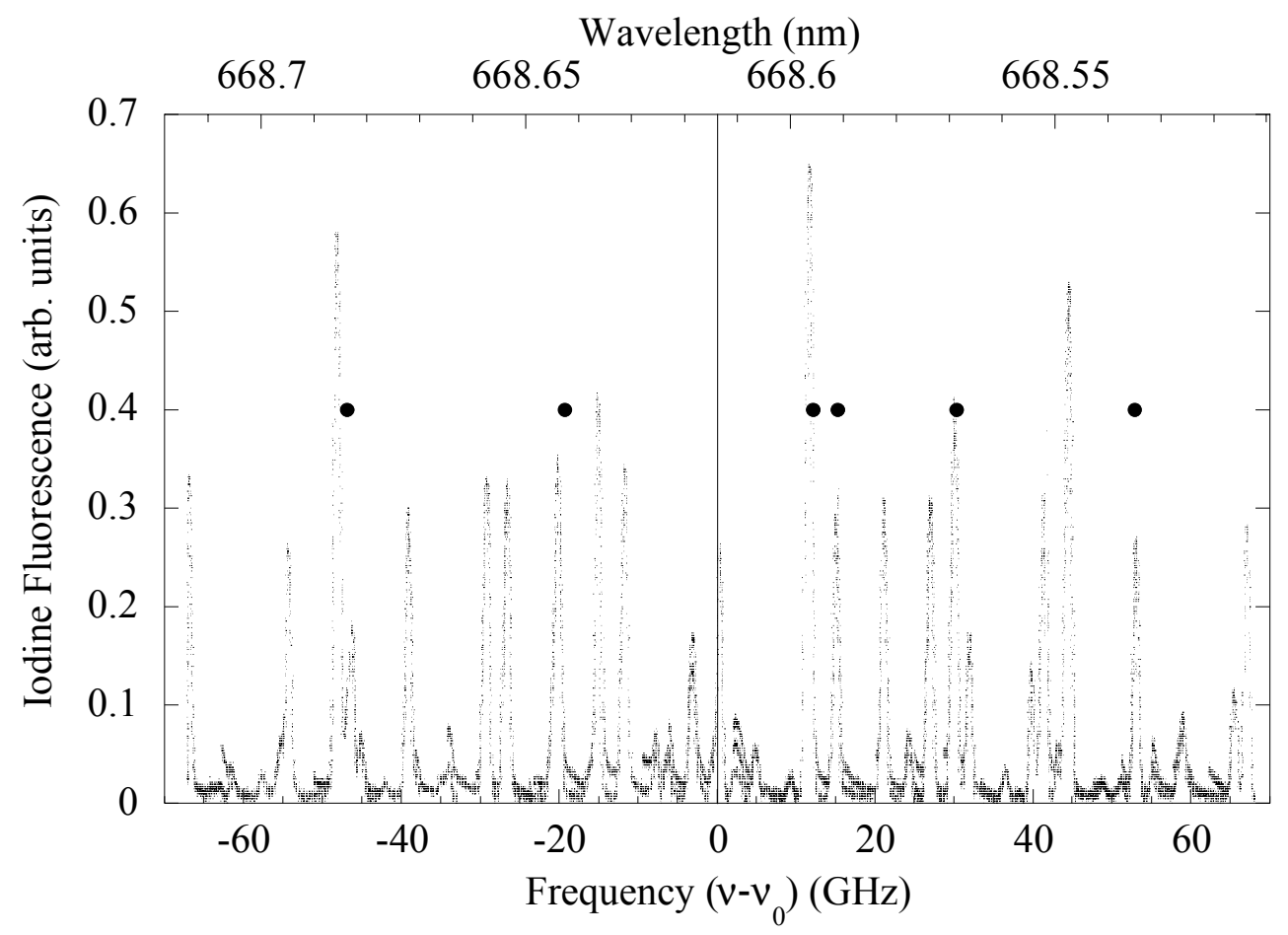

Figure 3.17 Iodine fluorescence spectrum for $60 \mathrm{GHz}$ on either side of the Ar II pump line. The vertical line at zero $\mathrm{GHz}$ indicates the Ar II pump line. The solid dots indicate iodine absorption lines in the published line atlas. 23

Recently, my iodine cell heater design was reproduced and similar measurements were made by Woo et al. ${ }^{24}$ They measured an iodine fluorescence pattern that matches Figure 3.16a, but found the wavelengths of the peaks to be shifted by $-0.002 \mathrm{~nm}$. It is possible that the calibration of our wavemeter is incorrect, which calls into question the exact location for the unshifted LIF absorption lines with respect to the iodine fluorescence spectrum. To determine this location for use in Ref. [14], G. Severn simultaneously measured etalon fringes and the iodine fluorescence as he scanned his diode laser (see Figure 3.18). ${ }^{25}$ The peaks in Severn's iodine fluorescence spectrum were matched with those calculated using a program based on calculations by Gerstenkorn and $L u c^{26}$ and provided to NIST (National Institute of Standards and Technology) by S. 
Gerstenkorn (see Table 3.3 below; original communication shown in Figure B.1). Severn then created a set of linear equations based on the simultaneous measurements which he solved to find an approximate location for the unshifted line. His result is indicated in Figure 3.18. The absolute wavelength values determined by the Severn result are closer to those of Woo et al. than ours (see Fig. 4 of Ref. [24]). Therefore, our absolute flow velocity calculations could be incorrect by as much as $0.002 \mathrm{~nm}$. A shift of $0.002 \mathrm{~nm}$ corresponds to an absolute velocity error of approximately $900 \mathrm{~m} / \mathrm{s}$.

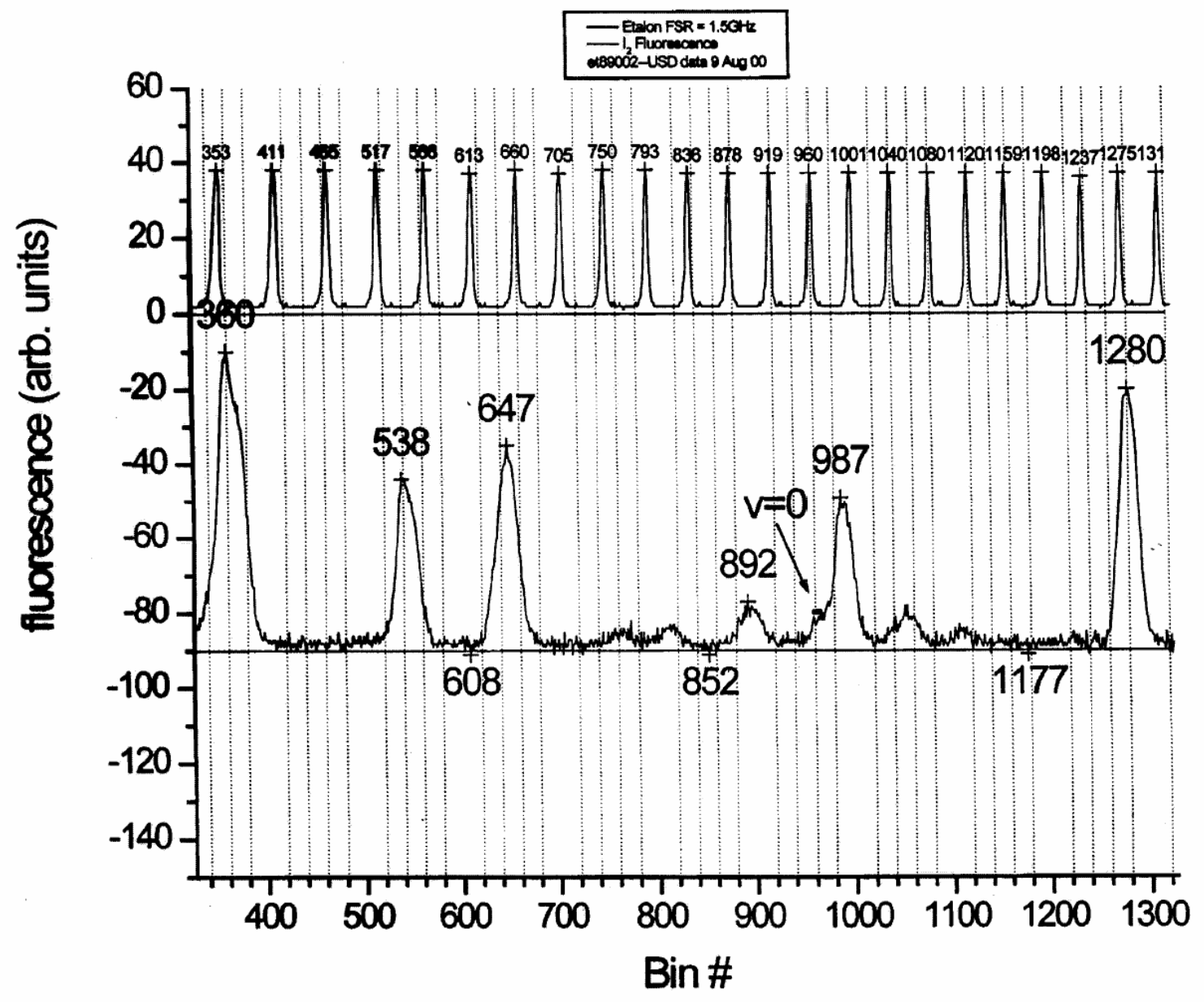

Figure 3.18 Iodine fluorescence versus etalon bin number as measured by G. Severn. Bin numbers of selected peaks are indicated as well as the calculated location of the unshifted $(v=0)$ Ar II LIF absorption line.

The iodine reference cell proved to be indispensable in the portability and 
reproducibility of the diode laser diagnostic system. Because it was too difficult to ship or carry a wavemeter to Australia for the experiments at the ANU, we arranged to use wavemeters owned by other labs at ANU. The two available wavemeters, a Burleigh WA-1100 and a Burleigh WA-4500, gave measurements of the diode laser wavelength that disagreed by $0.045 \mathrm{~nm}(\sim 33.5 \mathrm{GHz})$. The first unit is intended for use with lasers in the wavelength range $700-1650 \mathrm{~nm}$ and the second for a pulsed laser. Although both provided wavelength measurements of the $668 \mathrm{~nm}$ tunable diode laser, it is likely that using these wavemeters outside of their specifications caused an error in wavelength accuracy. To tune the laser to the appropriate wavelength for the argon ion LIF scheme, iodine fluorescence measurements were taken for a range of wavelengths until the pattern of peaks previously measured (see Figure 3.16a) was found. Neither wavemeter reported the same wavelengths for the iodine fluorescence peaks as measured at WVU. Using the WA-4500, the peaks were measured to be in the range of 668.56 to $668.58 \mathrm{~nm}$, which also does not agree with the Woo et al. results, suggesting that the iodine cell is a more accurate reference standard than typical commercial wavemeters.

Table 3.3 Iodine fluorescence lines provided to G. Severn by C. Sansonetti at NIST, including best match to Severn data, corresponding wavelength, and corresponding wavelength corrected for $-0.0056 \mathrm{~cm}^{-1}$ possible error in wave number.

\begin{tabular}{|l|c|c|c|c|}
\hline \multicolumn{1}{|c|}{ Classification } & $\begin{array}{c}\text { Calculated } \\
\text { wave number } \\
\left(\mathrm{cm}^{-1}\right)\end{array}$ & $\begin{array}{c}\text { Bin number } \\
\text { from Severn } \\
\text { measurement }\end{array}$ & $\begin{array}{c}\text { Wavelength } \\
(\mathrm{nm})\end{array}$ & $\begin{array}{c}\text { Corrected } \\
\text { wavelength } \\
(\mathrm{nm})\end{array}$ \\
\hline P 96 (3-5) & 14955.6711 & 360 & 668.64270 & 668.64294 \\
\hline P 81 (10-9) & 14955.7946 & & 668.63715 & 668.63739 \\
\hline R118(5-6) & 14955.8373 & 538 & 668.63525 & 668.63550 \\
\hline R 87(10-9) & 14955.8406 & & 668.63507 & 668.63531 \\
\hline R 55(6-7) & 14955.9440 & & 668.63049 & 668.63074 \\
\hline R139(11-9) & 14956.0280 & & 668.62671 & 668.62695 \\
\hline P122(7-7) & 14956.0768 & & 668.62451 & 668.62476 \\
\hline P 69(8-8) & 14956.1265 & & 668.62231 & 668.62256 \\
\hline P129(9-8) & 14956.2412 & 892 & 668.61719 & 668.61743 \\
\hline R135(9-8) & 14956.3223 & & 668.61359 & 668.61383 \\
\hline
\end{tabular}




\begin{tabular}{|l|l|l|l|l|}
\hline P 48 (6-7) & 14956.3445 & 987 & 668.61255 & 668.61279 \\
\hline R 75 (8-8) & 14956.4917 & & 668.60596 & 668.60620 \\
\hline P133(11-9) & 14956.5808 & & 668.60199 & 668.60223 \\
\hline P111(5-6) & 14956.7135 & 1280 & 668.59607 & 668.59631 \\
\hline R128(7-7) & 14956.7183 & & 668.59583 & 668.59607 \\
\hline
\end{tabular}

\subsection{Passive emission spectroscopy}

The various excited species in a plasma will undergo spontaneous de-excitation, emitting photons isotropically. The number of emission processes per second from an upper quantum level $k$ to a lower level $i$ is given by

$$
N_{k i}=n_{k} A_{k i}
$$

where $n_{k}$ is the number of atoms per cubic centimeter in level $k$ and $A_{k i}$ is the Einstein transition probability for spontaneous emission. ${ }^{27}$ This emission will be at a wavelength, $\lambda$, and the energy emitted is $h v$ times Equation 3.6, where $v$ is the frequency $(v=c / \lambda)$. The emissivity, defined as the radiant energy emitted by a unit volume per unit time, per unit of solid angle, is then given by

$$
\varepsilon_{\lambda}=\frac{h \nu}{4 \pi} A_{k i} n_{k}
$$

The line intensity (photon energy per second) is given by

$$
I_{\lambda}=\varepsilon_{\lambda} V \Omega
$$

where $V$ is the detected plasma volume and $\Omega$ is the solid angle subtended by the collection optics. ${ }^{28}$ The photonic current measured by the CCD on the monochromator (units of counts per second) is given by

$$
I_{p}(\lambda)=I_{\lambda} T_{\lambda} \Psi_{\lambda}
$$


where $T_{\lambda}$ is the transmission factor of the detection system, and $\Psi_{\lambda}$ is the sensitivity of the CCD. The sensitivity (measured in counts per Watt) is written as

$$
\Psi_{\lambda}=\eta_{\lambda} G / h v
$$

where $\eta_{\lambda}$ is the quantum efficiency of the CCD at wavelength $\lambda$ and $G$ is the gain of the detector.

\subsubsection{Spectroscopy diagnostic apparatus}

Measurements of spontaneous emission by excited neutral atoms used in this work were obtained from three different transitions: the $4 \mathrm{p}^{\prime}[1 / 2]_{0}$ to $4 \mathrm{~s}[3 / 2]^{\circ}{ }_{1}$ transition at $667.9126 \mathrm{~nm}$, the $4 \mathrm{p}^{\prime}[1 / 2]_{0}$ to the $4 \mathrm{~s}^{\prime}[1 / 2]^{\circ}{ }_{1}$ transition at $750.5934 \mathrm{~nm}$, and the $4 \mathrm{p}[1 / 2]_{0}$ to $4 \mathrm{~s}^{\prime}[1 / 2]^{\circ}{ }_{1}$ transition at $751.6720 \mathrm{~nm}$. These transitions are shown in Figure 3.19.

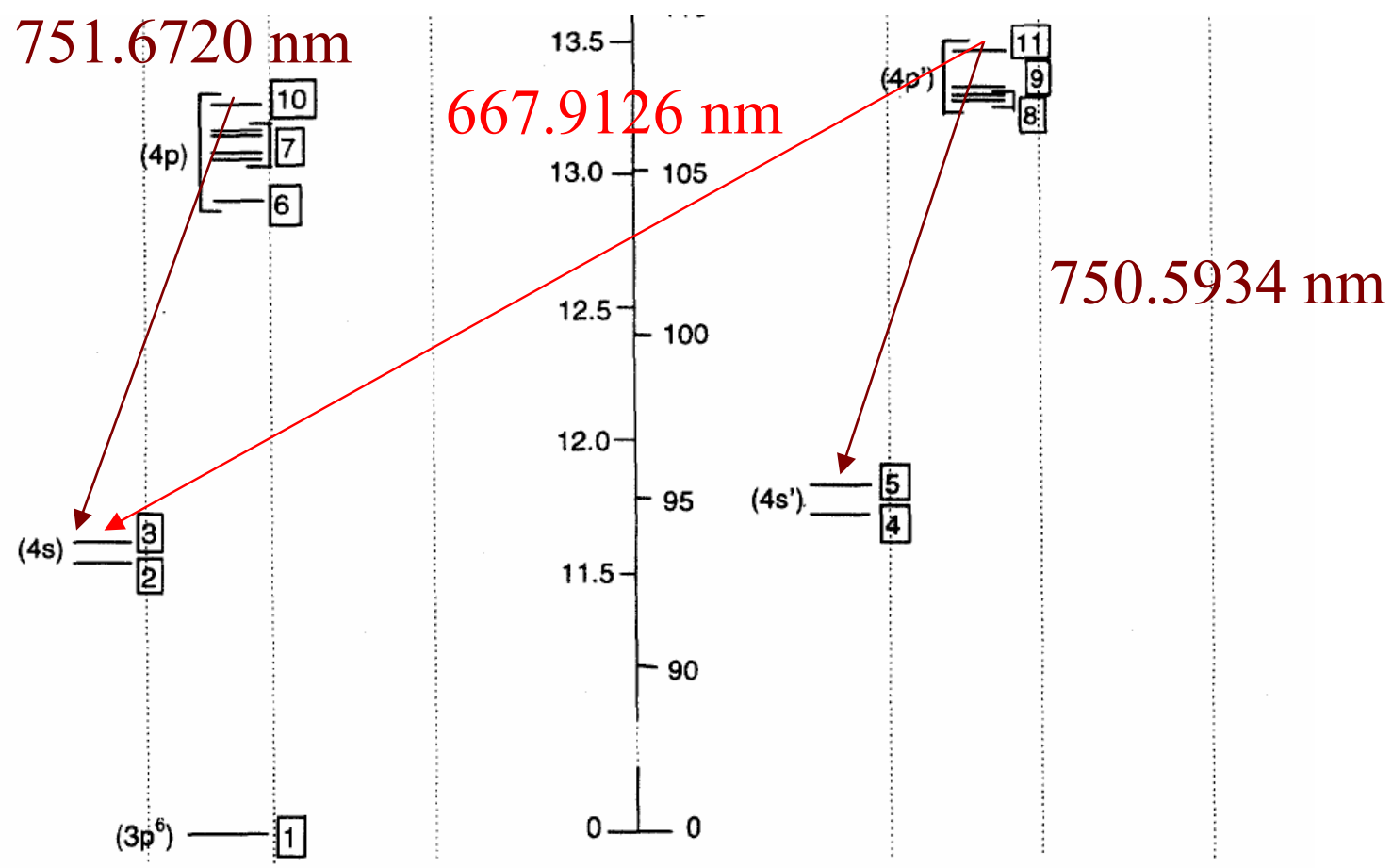

Figure 3.19 Diagram of the transitions used for passive emission spectroscopy.

Light emitted by spontaneous de-excitation is collected by a set of collimated optics, mounted on the vertical Velmex stage, which focuses it into a fiber optic cable 
attached to a McPherson ${ }^{\mathrm{TM}}$ Model 209 scanning monochromator. A fiber optics adapter is used to match the light from the fiber into the monochromator aperture. ${ }^{28}$ The monochromator consists of a two mirror, plane grating optical system, known as a Czerny-Turner system, with a 1.3 meter focal length. To select the desired detection wavelength, the grating is rotated while the mirrors and slits remain fixed. A CCD camera is attached to the exit slit for image capture. Relevant parameters of the monochromator are given in Table 3.4. ${ }^{29}$

Table 3.4 McPherson Model 209 scanning monochromator parameters.

\begin{tabular}{|l|l|}
\hline Parameter & Value \\
\hline $\mathrm{n}$ - grating groove density & $1200 \mathrm{gr} / \mathrm{mm}$ \\
\hline $\mathrm{F}-$ focal length & $1330 \mathrm{~mm}$ \\
\hline f-number & 11.6 (small grating) \\
\hline $\mathrm{D}-$ dispersion & $0362 \mathrm{~nm} / \mathrm{mm}$ \\
\hline $\mathrm{G}_{\mathrm{A}}-$ grating area & $102 \times 102 \mathrm{~mm}^{2}$ \\
\hline
\end{tabular}

The resolution of the spectrometer diagnostic is given by the full width at half maximum (FWHM) of a spectral line emitted by cold atoms measured with the smallest slit width that does not diffract the incoming light. This can be done by sending light from an argon calibration lamp through the fiber optic used for the diagnostic into the monochromator. The slit width is decreased until distortion in the measured spectral line can be seen. The resolution for the 667.9 and $750.6 \mathrm{~nm}$ lines was measured to be approximately $0.13 \mathrm{~nm}$. This corresponds to a width in frequency of $88 \mathrm{GHz}$, considerably larger than the $\sim$ few MHz resolution of the LIF diagnostic. 


\subsubsection{Abel inversion}

Abel inversion is a technique that can be used to find the radially dependent value of a cylindrically symmetric quantity at a given point from measurements that are average values of the quantity along chords through the plasma.

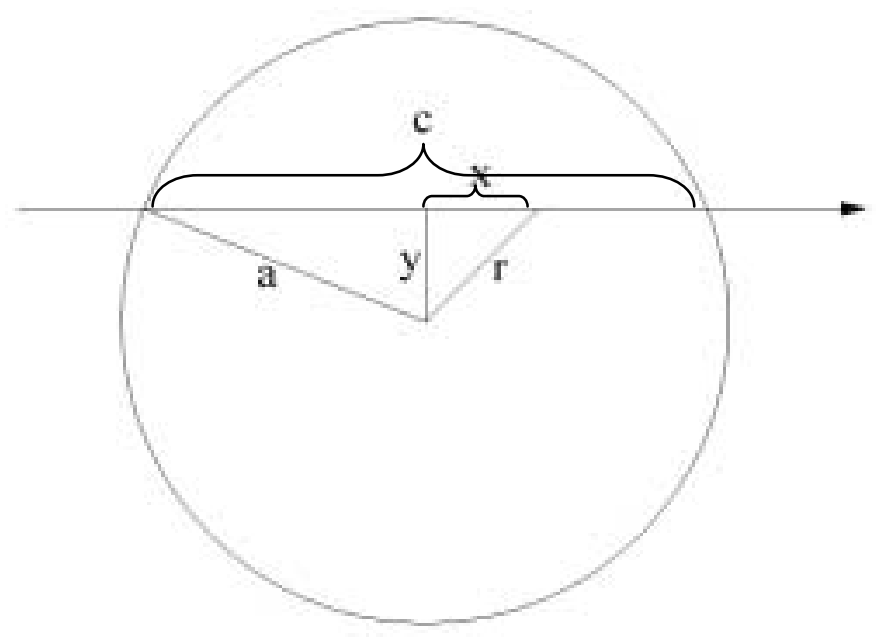

Figure 3.20 Geometry of cylinder of radius $a$ with chord of length $c$ at distance $y$ from the center.

The measured value, $F(y)$, can be written in terms of the cylindrically symmetric quantity, $f(r)$, as follows.

$$
F(y)=\int_{-\sqrt{a^{2}-y^{2}}}^{\sqrt{a^{2}-y^{2}}} f(r) d x
$$

Since $x^{2}+y^{2}=r^{2}$, a change of variables in the integral gives

$$
F(y)=\int_{-a}^{a} f(r) \frac{r d r}{\sqrt{r^{2}-y^{2}}}=2 \int_{y}^{a} f(r) \frac{r d r}{\sqrt{r^{2}-y^{2}}}
$$

known as the Abel transform. In order to find $\mathrm{f}(r)$ from $\mathrm{F}(y)$ measurements, the inverse Abel transform is needed. Since $\mathrm{f}(r)$ is zero for $r>a$, it is equivalent to extend the integral bound to infinity. 


$$
F(y)=2 \int_{y}^{\infty} f(r) \frac{r d r}{\sqrt{r^{2}-y^{2}}}
$$

Now let $\eta=y^{2}$ and $\rho=r^{2}$, so that $\mathrm{d} \rho=2 r \mathrm{~d} r$. Also define functions such that $\mathrm{F}(y)=\mathrm{F}_{\mathrm{a}}\left(y^{2}\right)$ $=\mathrm{F}_{\mathrm{a}}(\eta), \mathrm{f}(r)=\mathrm{f}_{\mathrm{a}}\left(r^{2}\right)=\mathrm{f}_{\mathrm{a}}(\rho)$, and $K(\eta)=\left\{\begin{array}{ll}-\eta^{-1 / 2} & \eta<0 \\ 0 & \eta>0\end{array}\right.$. Then the Abel transform equation becomes

$$
F_{a}(\eta)=\int_{0}^{\infty} K(\eta-\rho) f_{a}(\rho) d \rho
$$

The convolution theorem for Fourier transforms gives

$$
\widetilde{F_{a}}=\tilde{K} \widetilde{f_{a}}
$$

where the tilde $(\sim)$ indicates the Fourier transform. The Fourier transform of $K$ is given by $\tilde{K}(s)=\frac{1}{\sqrt{-i 2 s}}$, so that

$$
\widetilde{f_{a}}(s)=\sqrt{-i 2 s} \widetilde{F}_{a}(s)
$$

Now, multiplying Equation (3.16) by $\pi \sqrt{-i 2 s} / \pi \sqrt{-i 2 s}$, this becomes

$$
\widetilde{f_{a}}(s)=-\frac{1}{\pi} \frac{1}{\sqrt{-i 2 s}} i 2 \pi s \widetilde{F}_{a}(s)
$$

Since the Fourier transform for a derivative of a function is given by $g^{\prime}(x) \longrightarrow i 2 \pi s \tilde{g}(s)$, and again using the Fourier transform of $K$, we obtain

$$
f_{a}(\rho)=-\frac{1}{\pi} \int_{\rho}^{\infty} \frac{d F_{a} / d \eta}{(\eta-\rho)^{1 / 2}} d \eta
$$

Changing back to the original variables, this becomes

$$
f(r)=-\frac{1}{\pi} \int_{r}^{\infty} \frac{d F / d y}{\left(r^{2}-y^{2}\right)^{1 / 2}} d y=-\frac{1}{\pi} \int_{r}^{a} \frac{d F / d y}{\left(r^{2}-y^{2}\right)^{1 / 2}} d y
$$

where the last step is taken because $\mathrm{dF} / \mathrm{d} y=0$ for $y>a$. (This form assumes that $\mathrm{f}(r) \rightarrow 0$ 
at $r=a.)^{30}$

There are several methods for solving Eq. (3.19), including: ${ }^{27}$

1) integrating the equation graphically,

2) a. expanding Eq. (3.12) into a set of linear equations of the form

$$
F\left(y_{i}\right)=\sum_{j=i}^{N} a_{j i} f\left(r_{j}\right),
$$

b. creating a set of linear equations that uses an inverse matrix

$$
f\left(r_{j}\right)=\sum_{j=i}^{N} b_{j i} F\left(y_{i}\right)
$$

3) expanding the integral in Eq. (3.19) into a set of linear equations of the form

$$
f\left(r_{j}\right)=\sum_{j=i}^{N} \beta_{j i} F^{\prime}\left(y_{i}\right)
$$

4) using a polynomial approximation of $\mathrm{F}(y)$ and solving Eq. (3.19) using computer calculations.

Initially we used method 4 to invert our spectroscopic data. We fit a polynomial of the form

$$
F(y)=C_{1}+C_{2} \exp \left(-y^{2} / C_{3}\right)-C_{4} y^{2}
$$

to the data. Using the change of variables, $y=r \sec \theta$, Equation (3.19) becomes

$$
f(r)=-\frac{1}{\pi} \int_{0}^{\cos ^{-1}(r / a)} \frac{d F(r \sec \theta)}{d(r \sec \theta)} \sec \theta d \theta,
$$

where $a$ was chosen to be the largest integer such that $\mathrm{F}(a)>0$. A Mathematica program was used to solve Eq. (3.21) iteratively using 


$$
\begin{aligned}
& \operatorname{For}[j=1, j<100, j++ \\
& r[j]=a^{*} j / 100 \\
& e[j]=N\left[-(1 / P i) \int_{0}^{\operatorname{ArcCos} r[[j] / a]} f f^{\prime}[r[j] \operatorname{Sec}[\theta]] \operatorname{Sec}[\theta] d \theta\right]
\end{aligned}
$$

].

This method yields an array, $e[j]$, corresponding to the radially dependent $\mathrm{f}(r)$. Unfortunately, this method has several disadvantages. Any radial structure information inherent in the deviations of the measurements from the fit polynomial is lost, i.e., the polynomial fitting method forces the assumption of a source profile that is monotonically increasing toward the center of the plasma.

After presenting results using the above method at a conference, R.E. Bell suggested a matrix inversion method (method $2 b$ ) that preserves detailed radial structure information. This method involves dividing the viewing region of the plasma into several segments and determining, geometrically, how much each region contributes to a given line of sight measurement. ${ }^{31}$ The measured plasma cross-section was divided into concentric circles such that each circle is considered to be an "emission zone" where the emission is the same from anywhere in that zone. A length matrix, $\mathbf{L}$, can then be created where each element $L_{i j}$ describes the length of line of sight $i$ that passes through emission zone $j$. The measured quantity, $F$, will then be related to the quantity, $f$, emitted by each emission zone by

$$
F_{i}=\sum_{j} L_{i j} f_{j} .
$$

The desired quantity, $f$, can then be determined by inverting matrix $\mathbf{L}$ and calculating

$$
f_{i}=\sum_{j} L_{i j}^{-1} F_{j} .
$$

The $k$ emission zones, where $k$ is the number of measurements made from the 
center to edge of the plasma, are created such that the radius of zone $i$ lies halfway between the line of sight of measurement $i$ and measurement $i+1$, as shown in Figure 3.21 for $k=5$. If $y_{i}$ is the position of each line of sight measurement, then the radius of each emission zone circle is defined by

$$
r_{i}=\left(y_{i}+y_{i+1}\right) / 2
$$

and $r_{k}=R_{\max }$.

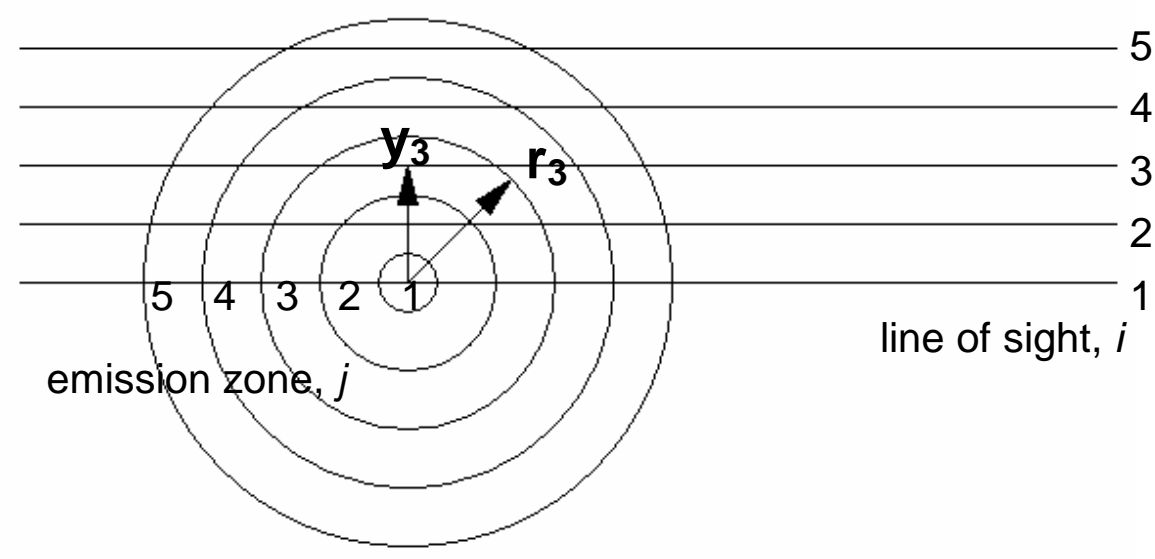

Figure 3.21 The plasma cylinder is divided into emission zones that are concentric circles such that the radius $r_{j}$ of zone $j$ lies halfway between lines of sight $j$ and $j+1$. The line of sight $i$ is located $y_{i}$ from the center.

The elements of the length matrix can be calculated by finding the length of a chord $c$ across a circle of radius $a$ at a distance $y$ from the center of the circle. As shown in Figure 3.20, these are related by $c / 2=\sqrt{a^{2}-y^{2}}$. The matrix elements are then given by

$$
\begin{aligned}
& \text { if } r_{j}>y_{i} \text {, and } i=j, L_{i j}=2 \sqrt{r_{j}^{2}-y_{i}^{2}}, \\
& \text { if } r_{j}>y_{i} \text {, and } i \neq j, L_{i j}=2 \sqrt{r_{j}^{2}-y_{i}^{2}}-2 \sqrt{r_{j-1}^{2}-y_{i}^{2}}, \\
& \text { if } r_{j}<y_{i}, L_{i j}=0 .
\end{aligned}
$$


For our experiments, $k=15$ and $R_{\max }=3.625 \mathrm{~cm}$ is set by the diameter of the window through which the measurements are performed. The radius of the plasma is actually larger than $3.625 \mathrm{~cm}$, but this value of $R_{\max }$ is chosen so that the width of the outermost emission zone used in the inversion is the same width as the other zones. Since the viewport size limits our emission collection much beyond $R_{\max }$ in the vertical direction, it is impossible to include the contribution from that region in the calculation. However, emission from outside $R_{\max }$ does contribute along each horizontal line of sight that includes plasma radii larger than $R_{\max }$. Therefore, the outermost emission zone includes contributions that are not correctly weighted, i.e., if the emission intensity was constant across the plasma cross section, even after accounting for the longer line of sight through the outer emission zones, the measured emission from the outermost zone would be higher than the emission from the inner zones because of the additional contributions from plasma beyond $R_{\max }$. A Matlab ${ }^{\circledR}$ code was used to calculate the matrix and perform the inversion. A comparison of the two methods and the collisional-radiative model profile to be matched is shown in Figure 3.22, where the inversion results have been normalized to the model output. The advantage of the matrix inversion method's ability to yield non-monotonically increasing results can be seen at $r=1.5$. 

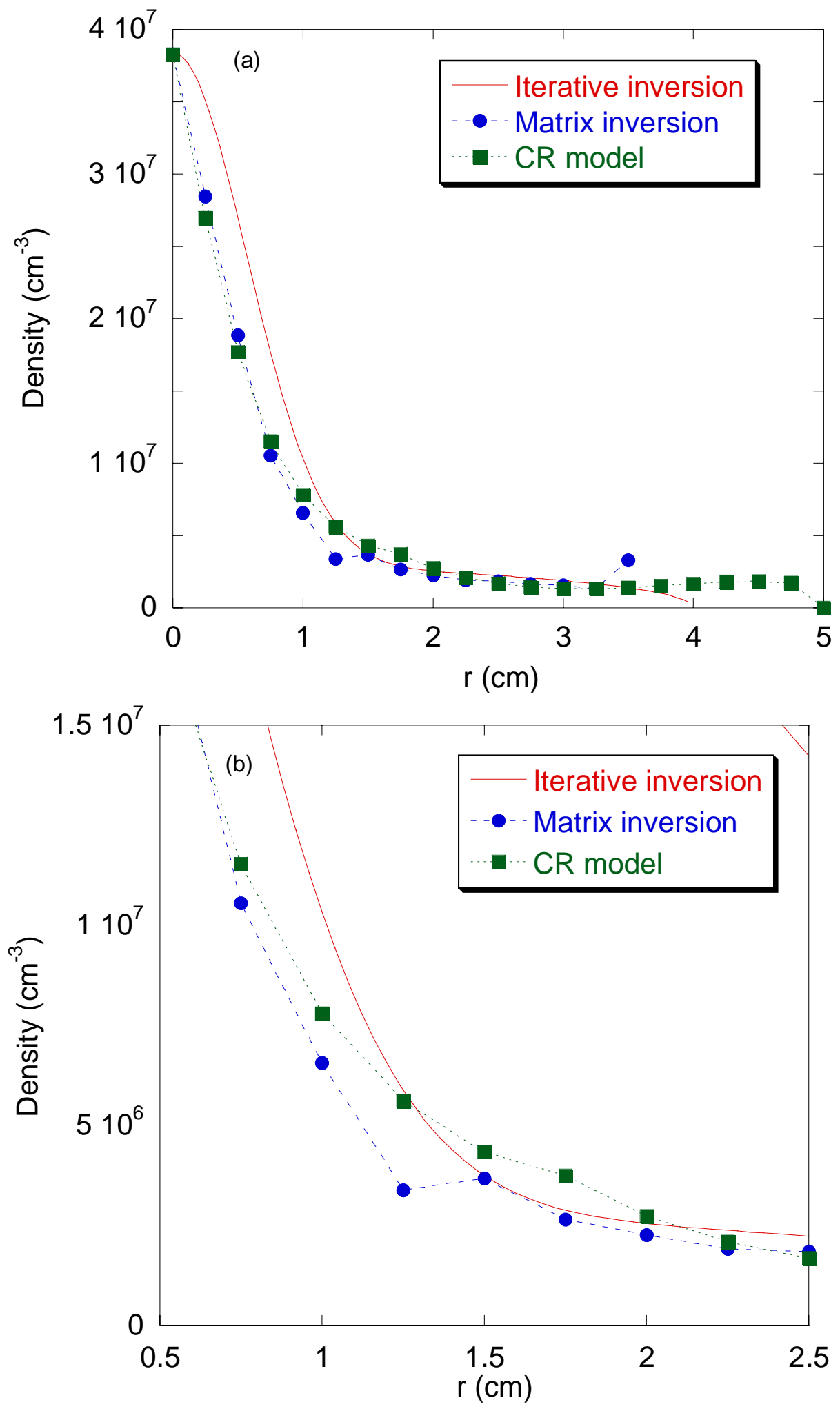

Figure 3.22 Comparison of iterative (solid line) and matrix (circles) Abel inversion techniques. The relevant CR model profile (squares) is also shown for reference. Part b) is a zoomed version of part a) for $0.5<r<2.5$ to illustrate the non-monotonic radial variation that is retained in the matrix inversion but not in the iterative inversion. 


\subsection{Langmuir probe}

Perhaps the oldest and most basic of plasma diagnostics is the Langmuir probe, an electrical probe used extensively by Irving Langmuir. Electrical probes are used to measure the charge carrier concentration, the plasma potential, and the velocity distribution of electrons. A Langmuir probe consists of a conducting material inserted into the plasma to give local measurements at the probe tip. Because the probe is in the plasma, one must consider the perturbative effects of the probe on the plasma. ${ }^{32}$

In a typical two-species plasma, the electrons have a faster mean speed than the ions due to their lighter mass. Thus, if an electrically insulated probe is inserted into a plasma, it would charge negatively due to the larger electron flux to the probe. Eventually it would reach a negative charge such that no more electrons are drawn to it, yielding a zero net current. The potential at which this occurs is called the floating potential. If the probe is then connected to a voltage source, the voltage on the probe can be manipulated to draw current from the plasma.

Applying a potential to the probe that is more negative than the floating potential attracts ions, yielding a net positive current. As the potential becomes more negative, there will be a point at which the ion current is maximized, known as ion saturation current. Conversely, applying a more positive potential than the floating potential to the probe will draw electron current. When the collected electrons are maximized, it is called electron saturation current. The plasma potential is the potential at which the electron saturation current just begins.

Such measurements are typically plotted as a current versus potential plot, know as an I-V trace. A hypothetical trace is shown in Figure 3.23. The sign convention used 
for current is that of current flowing out of the probe. The locations of floating and plasma potentials, and ion and electron saturation currents are indicated.

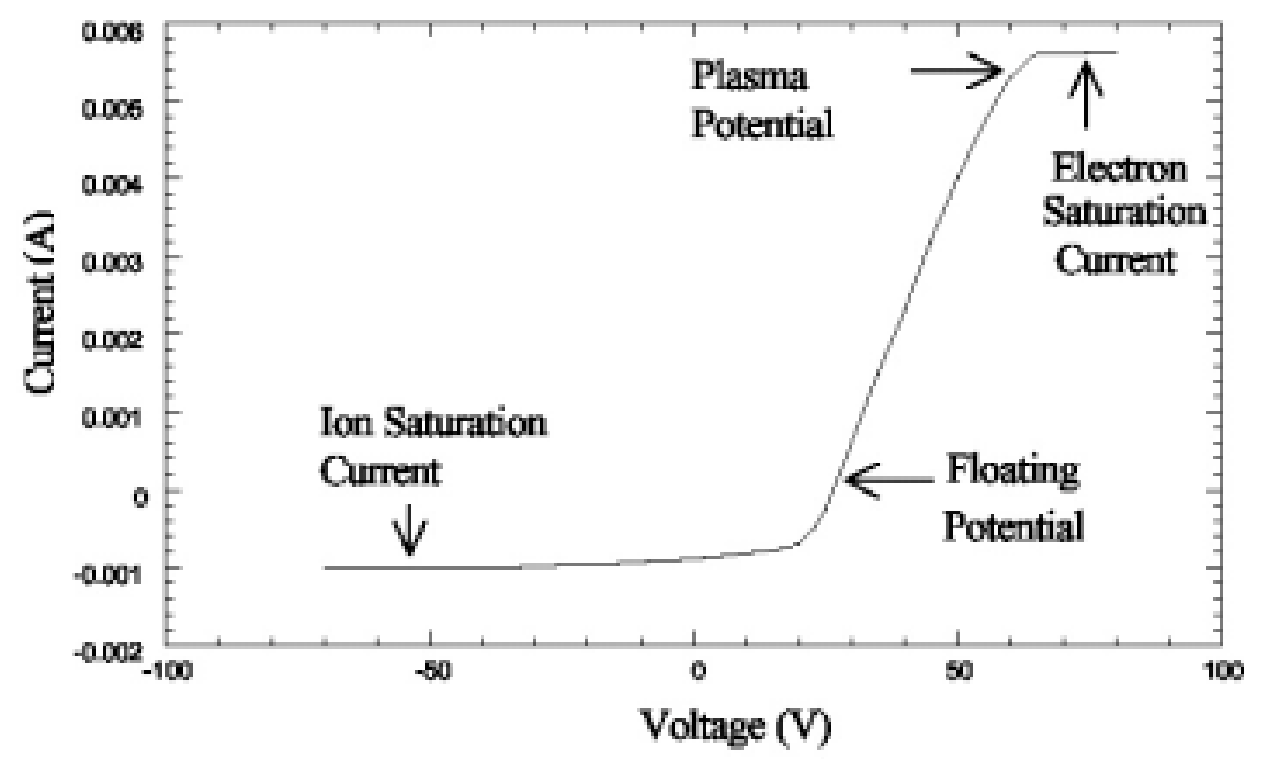

Figure 3.23 Langmuir probe I-V trace. ${ }^{33}$

To determine plasma parameters from the I-V trace, the electrons are assumed to be Maxwellian. The total electrical current from the probe is given by

$$
I=n_{e} e A_{p}\left(\frac{T_{e}}{m_{i}}\right)^{1 / 2}\left[\frac{1}{2}\left(\frac{2 m_{i}}{\pi m_{e}}\right)^{1 / 2} \exp \left(\frac{e V_{0}}{T_{e}}\right)-\frac{A_{s}}{A_{p}} \exp \left(-\frac{1}{2}\right)\right],
$$

where $\mathrm{n}$ is the plasma density, $A_{p}$ is the surface area of the probe, $T_{e}$ is the electron temperature, $V_{0}$ is the voltage seen by the plasma (applied voltage minus plasma potential, $V-V_{p}$ ), and $A_{s}$ is the area of the sheath surface. ${ }^{32}$ The second term in the brackets is the ion saturation current, $I_{s i}$, which, for an unmagnetized plasma, is given by

$$
I_{s i}=-e J_{i}=-0.61 e n_{e} A_{p} \sqrt{T_{e} / m_{i}} .
$$

The slope of the I-V trace is given by

$$
\frac{d I}{d V_{0}}=\frac{e}{T_{e}}\left(I-I_{s i}\right)+\frac{d I_{s i}}{d V_{0}}
$$


As can be seen in the I-V trace, $d I_{s i} / d V_{0} \ll d I / d V_{0}$, so that $T_{e}$ can be approximated by

$$
T_{e}=e\left(I-I_{s i}\right) / \frac{d I}{d V_{0}} .
$$

Experimentally, a line is fit to $\ln \left|I-I_{s i}\right|$ versus $V_{0}$ in order to determine the slope which is the inverse of $T_{\mathrm{e}}$. Once $T_{e}$ is known, Equation 3.27 can be used to determine the plasma density, $n_{e}$.

Because of the high densities in helicon sources, Langmuir probes are typically not driven into the electron saturation current region because the probe would not have a sufficiently negative potential to form a sheath and the high heat flux could destroy the probe. ${ }^{32}$ Without the electron saturation current measurement, we are unable to measure the plasma potential directly. The voltage $V_{0}\left(V_{0}=V-V_{p}\right)$ must then be approximated by the applied voltage in the above calculations. Since the plasma potential is constant, the slope measured will not be affected by this approximation, yielding the same measurement of electron temperature. ${ }^{5}$

Because ions and electrons gyrate around magnetic field lines, the cross-field motion of the particles will be restricted, affecting the amount of particles reaching a Langmuir probe in a magnetized plasma. The effect will be determined by the relationship between the gyro-radius of each species and the size of the probe. Because the electrons have a smaller gyro-radius than the ions, the motion of the electrons will be reduced with respect to the ions, which will decrease the electron saturation current. However, since we do not drive the probe into the electron saturation current region, the probe remains significantly negative such that the electron dynamics can be treated as described above. ${ }^{32}$ The ion gyro-radius in HELIX with a $1000 \mathrm{G}$ magnetic field and an 
ion temperature of $0.3 \mathrm{eV}$ is approximately $3.5 \mathrm{~mm} .{ }^{5}$ Since this is on the order of the probe tip length of $2.0 \mathrm{~mm}$, we must consider the effects of the magnetic field on the ions. The magnetic field reduces the number of ions reaching the probe, such that Equation 3.27 becomes $^{32}$

$$
I_{s i}=-0.49 e n_{e} A_{p} \sqrt{T_{e} / m_{i}} .
$$

The RF fields in helicon source plasmas will also affect Langmuir probe measurements. The fields will accelerate and decelerate the electrons toward the probe when the probe potential is near the floating potential, yielding an error in floating potential measurement. ${ }^{34}$ Sudit and Chen developed a method of RF compensation for Langmuir probes ${ }^{35}$ which we use in our probe design. A floating electrode is exposed to the plasma potential fluctuations and is connected to the probe tip by a large capacitor, which lowers the sheath impedance such that the probe tip will follow the plasma potential oscillations. Additionally, a chain of RF chokes is connected to the probe tip which increases the impedance of the circuit at the RF frequency.

A schematic drawing of the Langmuir probe design is shown in Figure 3.24. The probe tip is $0.5 \mathrm{~mm}$ diameter graphite (mechanical pencil material) inserted into a $0.6 \mathrm{~mm}$ diameter alumina shaft and attached by a set screw to a copper base. A $10 \mathrm{nF}$ capacitor is also connected to the copper base. This assembly is placed inside a boron nitride (BN) cap such that the probe tip extends into the plasma through a hole in the BN cap, and the opposite leg of the capacitor remains within the head so that it is not directly exposed to the plasma. The threaded BN cap attaches to the stainless steel probe shaft. The chain of RF chokes is attached to the copper base. The RF chokes are $1 / 4$ Watt shielded inductors, each specially designed to shield a certain RF frequency, from Lenox-Fugle International, 
Inc. ${ }^{36}$ Starting from the copper base, the RF chokes are ordered as follows: 26, 53, 26, 13.2, 6.8 MHz. These are then connected to a probe wire that is attached to the BNC vacuum feedthrough at the rear of the probe shaft. Solder is used to create electrical connections among the components. Thermaflex tubing is used to cover the chain of RF chokes. Figure 3.25 is a picture of the Langmuir probe head. Additional details of the Langmuir probe design can be found in Refs. [4, 5, and 37].

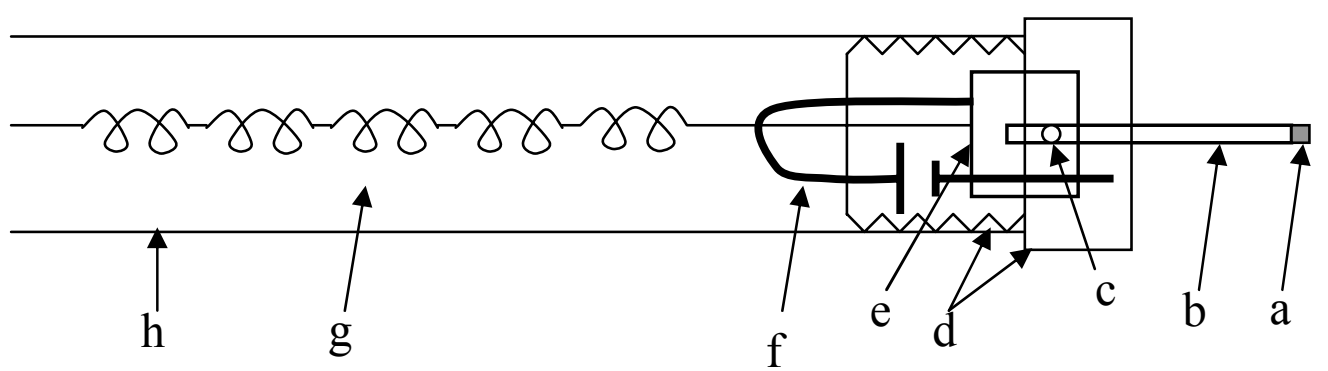

Figure 3.24 Schematic drawing of Langmuir probe design including a) graphite probe tip, b) alumina shaft, c) set screw, d) threaded boron nitride cap e) copper base, f) capacitor, g) chain of RF chokes, and h) stainless steel probe shaft. 


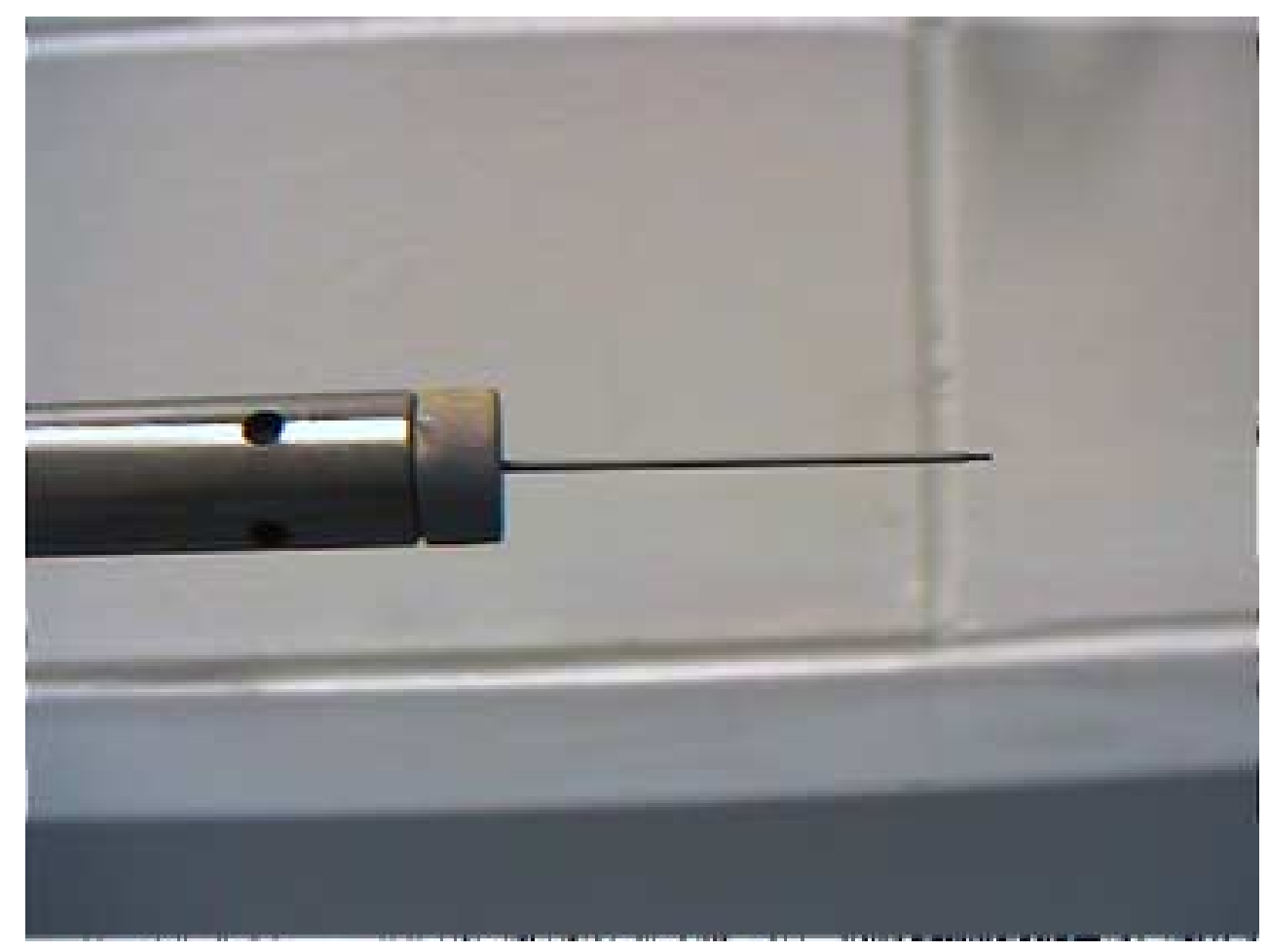

Figure 3.25 Photograph of the Langmuir probe head. The exposed graphite tip is $2 \mathrm{~mm}$ long and runs the length of the alumina tube into the boron-nitride cap. ${ }^{37}$

A Keithley 2400 SourceMeter is used to measure the Langmuir probe I-V trace. The source meter applies the voltage to the probe as well as measuring the collected current. The source meter is controlled by custom software created with LabWindows ${ }^{\mathrm{TM}}$ via a GPIB interface. The Langmuir probe measurements used in these experiments were obtained $50 \mathrm{~cm}$ downstream of the antenna (location B in Figure 2.2). 


\section{References}

${ }^{1}$ R.A. Stern and J.A. Johnson, Phys. Rev. Lett. 34, 1548 (1975).

${ }^{2}$ H.C. Meng and H.-J. Kunze, Phys. Fluids 22, 1082 (1979).

${ }^{3}$ D.N. Hill, S. Fornaca, and M.G. Wickham, Rev. Sci. Instrum. 54, 309 (1983).

${ }^{4}$ M.M. Balkey, Optimization of a Helicon Plasma Source for Maximum Density with Minimal Ion Heating, Ph.D. Dissertation, West Virginia University, Morgantown (2000).

${ }^{5}$ J.L. Kline, Slow Wave Ion Heating and Parametric Instabilities in the HELIX Helicon Source, Ph.D.

Dissertation, West Virginia University, Morgantown (2002).

${ }^{6}$ J.C. Camparo, Contemp. Phys. 26, 443 (1985).

${ }^{7}$ B. Bölger and J.C. Diels, Phys. Lett. A 28, 401 (1968).

${ }^{8}$ C.E. Wieman and L. Holberg, Rev. Sci. Instrum. 62, 1 (1991).

${ }^{9}$ M.G. Littman and J. Metcalf, Appl. Opt. 17, 2224 (1978).

${ }^{10}$ M. Born and E. Wolf, Principles of Optics (Cambridge U.P., Cambridge, 1959).

${ }^{11} \mathrm{http} / / / \mathrm{www}$. sacher-laser.com/ar_diode.php.

${ }_{13}^{12}$ R.F. Boivin and E.E. Scime, Rev. Sci. Instrum. 74, 4352 (2003).

${ }^{13}$ J. Sacher, private communication, 27 July 2005.

${ }^{14}$ G.D. Severn, D.A. Edrich, and R. McWilliams, Rev. Sci. Instrum. 69, 10 (1998).

${ }^{15}$ Sacher Lasertechnik LLC, Instruction Manual, Hannah Arendt Str. 3-7 D35037 Marburg/Lahn Germany (2000).

${ }^{16} \mathrm{http}: / /$ physics.nist.gov/PhysRefData/ASD/index.html

${ }^{17}$ W.L. Wiese, M.W. Smith, and B.M. Glennon, Atomic Transition Probabilities, NSRDS-NBS (1966).

${ }^{18}$ M.J. Goeckner and J. Goree, J. Vac. Sci. Technol. A 7, 977 (1989).

${ }^{19}$ R.F. Boivin, "Study of the Different Line Broadening Mechanisms for the Laser Induced Fluorescence Diagnostic for the HELIX and LEIA Plasmas," PL-039 (1998).

${ }^{20}$ R.F. Boivin, "Zeeman Splitting for LIF Transitions and De-convolution Technique to Extract Ion Temperature,” PL-050, EPAPS-E-PHPAEN-10-003306 (2001).

${ }^{21}$ L. Pauling and S. Goudsmit, The Structure of Line Spectra (McGraw-Hill, New York, 1930).

${ }^{22}$ G. Marr, Plasma Spectroscopy, (Elsevier, 1968).

${ }^{23} \mathrm{~S}$. Gerstenkorn and P. Luc, Atlas Du Spectre D'absorption De La Molecule D'iode, Editions DuCentre National De La Recherche Scientifique (1978).

${ }^{24}$ H.-J. Woo et al., Journal of the Korean Physical Society 48, 260 (2006).

${ }^{25} \mathrm{G}$. Severn, private communication.

${ }^{26}$ S. Gerstenkorn and P. Luc, J. Physique 46, 867 (1985).

${ }^{27}$ W. Lochte-Holtgreven, Plasma Diagnostics, (American Institute of Physics, New York, 1995).

${ }^{28}$ R.F. Boivin, "Spectroscopy System and Basic Spectroscopy Diagnostics for the HELIX and LEIA Plasma Devices," PL-046 (2000).

${ }^{29}$ McPherson, Instruction Manual for the Model 209 1.33 Meter Scanning Monochromator (1998).

${ }^{30}$ R.N. Bracewell, The Fourier Transform and Its Applications, (McGraw-Hill, New York, 1978).

${ }^{31}$ R.E. Bell, Rev. Sci. Instrum. 66, 558 (1995).

${ }^{32}$ I.H. Hutchinson, Principles of Plasma Diagnostics, (Cambridge U.P., Cambridge, 1987).

${ }^{33}$ P.A. Keiter et al., Phys. Plasmas 7, 779 (2000).

${ }^{34}$ N.A. Krall and A.W. Trivelpiece, Principles of Plasma Physics, (San Francisco Press, San Francisco, 1986).

${ }^{35}$ I.D. Sudit and F.F. Chen, Plasma Sour. Sci. Technol. 3, 162 (1994).

${ }^{36}$ Lenox-Fugle International, Inc. 1071 N. Grand Ave. \#115, Nogales, AZ 85621. (520) 287-5847.

${ }^{37}$ P.A. Keiter, Experimental Investigation of Ion Temperature Anisotropy Driven Instabilities in a High Beta Plasma, Ph.D. Dissertation, West Virginia University, Morgantown (1999). 


\section{Chapter 4: ANU Experiments}

\subsection{Introduction}

To better compare the double layer (DL) measurements performed using LIF on HELIX/LEIA at WVU and MNX at PPPL with those using the retarding field energy analyzer (RFEA) on Chi-Kung at ANU, we were interested in performing LIF measurements on Chi-Kung. Additionally, the RFEA measurements are limited in spatial resolution (the RFEA can only be placed at discrete locations along the axis of the experiment). There was also a strong possibility that the grounded RFEA significantly perturbed the plasma during the measurement, whereas LIF could provide nonperturbative, spatially resolved measurements of the ion velocity distribution function in the Chi-Kung plasma. I was awarded funding through the East Asia and Pacific Summer Institute (EAPSI) Program sponsored by the National Science Foundation and the Australian Academy of Science to travel to Australia and perform these measurements. The portability of the diode laser LIF diagnostic was essential to this collaboration. (For more information on International Equipment Transit, see Appendix C.)

Typical normalized RFEA ion energy distribution function (IEDF) measurements in the Chi-Kung plasma downstream of the region of diverging magnetic field are shown in Figure 4.1. The two ion populations evident in Figure 4.1 (the two peaks in the IEDF) are the fast ion beam flowing away from the source and the stationary ion population trapped downstream by the strong plasma potential gradient of the DL. ${ }^{1}$ The trapped ion population appears in the grounded RFEA measurement at an energy equal to the local plasma potential while the ion beam appears at higher energy. 

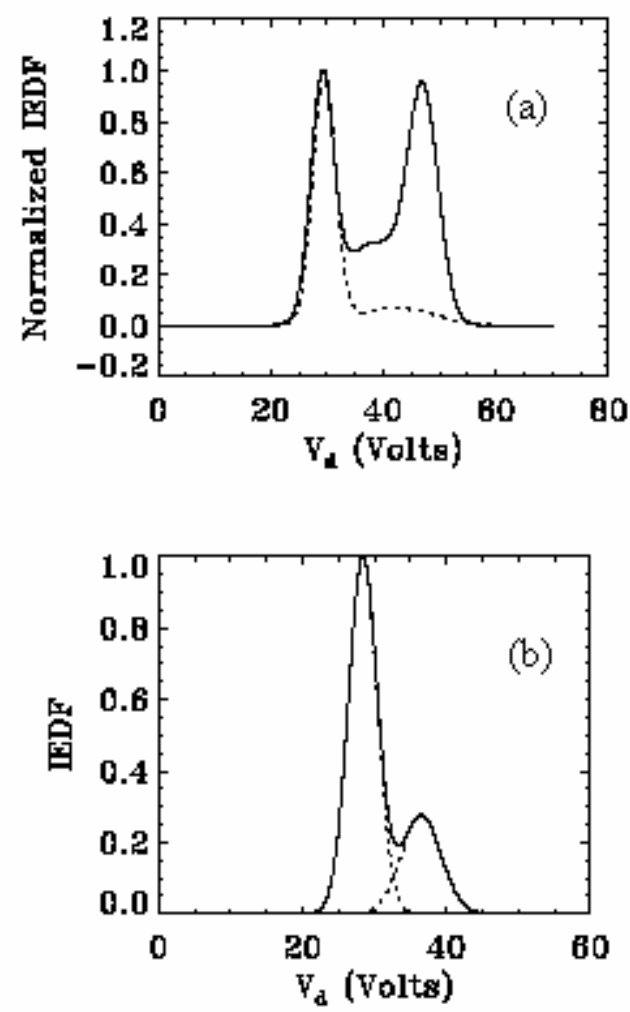

Figure 4.1 Normalized IEDFs obtained with the RFEA at $\mathrm{P}=250 \mathrm{~W}$ and the strong magnetic field case. (a) $z=37 \mathrm{~cm}$ (solid line) and $z=50 \mathrm{~cm}$ (dashed line) for $\mathrm{P}=0.35$ mTorr. ${ }^{1}$ (b) $\mathrm{z}=37 \mathrm{~cm}$ for $\mathrm{P}=1 \mathrm{mTorr}$. The dashed line is a Gaussian fit to each peak.

In the Cohen et al. LIF measurements of parallel ion flow speeds in MNX, for a neutral pressure of $0.6 \mathrm{mTorr}$ in the main chamber, an ion beam of energy of $17 \mathrm{eV}$ (corresponding to a beam speed of $9055 \mathrm{~m} / \mathrm{s}$ ) was observed. ${ }^{2}$ Cohen et al. observed an exponential decrease in the LIF signal from the ion beam as a function of distance from the DL - even though the ion beam energy remained constant or increased slightly with distance from the DL. Analysis of the MNX measurements indicated that the decrease in LIF signal arose from the collisional depletion of the metastable ion states probed in the LIF measurement process, i.e., the beam continued to propagate into the diffusion region but the metastable ions in the beam needed for the LIF measurement were rapidly quenched. 
In contrast to the MNX experiments, the Sun et al. experiments were performed in the freely expanding HELIX helicon plasma (whereas the expanding MNX plasma was channeled through a small aperture and a strong magnetic nozzle). With a geometry very similar to the Chi-Kung apparatus, high speed ion flows were also observed below a critical neutral pressure. Subsequent LIF and Langmuir probe measurements in HELIX provided detailed information about the spatial structure and magnitude of the electric field in the ion acceleration region. ${ }^{3}$ Consistent with the predictions of the onedimensional particle-in-cell (PIC) computer code that simulated an expanding, currentfree plasma ${ }^{4}$ the later HELIX measurements indicated that the ions accelerated through the pre-sheath of the DL and, once in the DL proper, the ions rapidly accelerated to supersonic speeds. Downstream of the DL, a single Maxwellian, trapped population of ions was observed.

\subsection{Experimental apparatus}

The Chi-Kung helicon source ${ }^{5}$ (Figure 4.2a) consists of a $23 \mathrm{~cm}$ long, $15 \mathrm{~cm}$ diameter glass tube terminated at one end by a $1 \mathrm{~cm}$ thick glass plate. Attached to the tube opposite of the plate is a grounded aluminum diffusion chamber, $30 \mathrm{~cm}$ long with a $32 \mathrm{~cm}$ diameter. RF power at $13.56 \mathrm{MHz}$ is supplied through a matching network to a 20 $\mathrm{cm}$ long double saddle antenna wrapped around the glass tube. Gas, typically argon, is introduced through the side of the diffusion chamber. A base pressure of $2 \times 10^{-6} \mathrm{mTorr}$ is obtained by a turbomolecular/rotary pumping system connected to the wall of the diffusion chamber. The magnetic field is created by two co-axial, solenoidal coils placed at each end of the source tube. Varying magnetic field strengths and profiles were created by independently adjusting the current to each coil. The highest field case investigated 
(peak field of $140 \mathrm{G}$ ) is obtained for a current of 6 Amps in both coils. There are no coils around the diffusion chamber, yielding a field geometry as shown in Figure $4.2 \mathrm{~b}$ for the strong magnetic field case. Neutral pressure in the diffusion chamber is measured with an ion gauge and a Baratron ${ }^{\circledR}$ gauge.

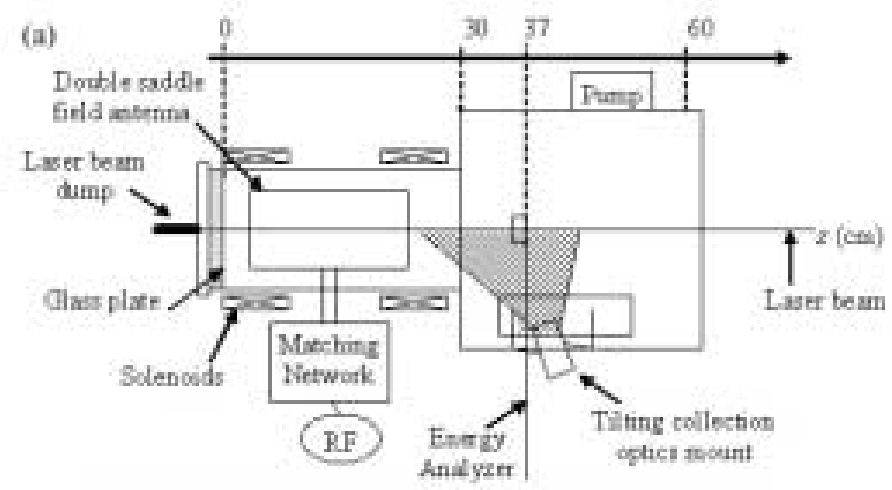

(b)

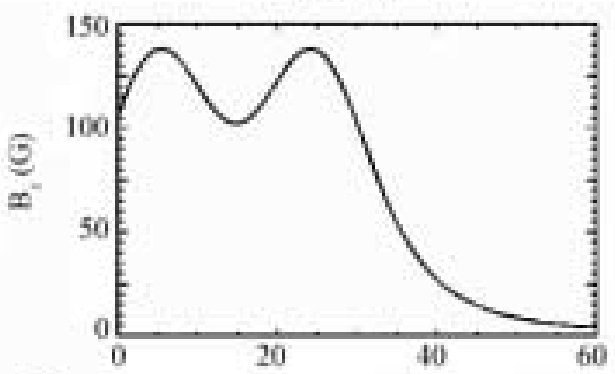

(c)

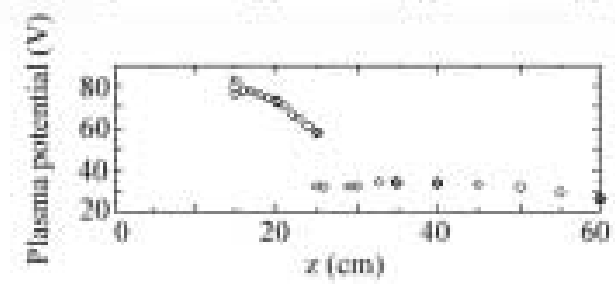

Figure 4.2 (a) Chi-Kung apparatus with field of view of LIF collection optics shown as the shaded region. (b) Magnetic field geometry shown for strong magnetic field ( $I_{B}=6 \mathrm{~A}$ in both coils) case. (c) Plasma potential versus axial position in Chi-Kung showing double layer potential drop for $P=0.2$ mTorr, Power $=250 \mathrm{~W}$, and the strong magnetic field case. ${ }^{6}$

For neutral pressures less than about 1.0 mTorr, a strong gradient in the plasma potential spontaneously appears near the end of the helicon plasma source. The plasma potential as a function of axial position is shown in Figure 4.2c for a pressure of 0.2 mTorr. The RFEA probe provides measurements of the local plasma potential and the 
ion energy distribution function (IEDF). Two such IEDF measurements are shown in Figure 4.1. The IEDF measured at $z=37 \mathrm{~cm}$ (solid line) and $z=50 \mathrm{~cm}$ (dashed line) for a pressure of $0.35 \mathrm{mTorr}$ are shown in Figure 4.1a. At $z=37 \mathrm{~cm}$, the ion beam is approximately $9300 \mathrm{~m} / \mathrm{s}$ faster than the trapped ion population. (RFEA measurements assume that the background population is at rest to calculate the plasma potential. Thus, the beam velocity measurement can only be given relative to the background population.) As the neutral pressure increases, both the energy and density of the ion beam decrease. The normalized IEDF for a pressure of 1.0 mTorr at $z=37 \mathrm{~cm}$ is shown in Figure $4.1 \mathrm{~b}$. At this higher pressure, the beam is approximately $6200 \mathrm{~m} / \mathrm{s}$ faster than the background population. According to previous RFEA measurements, collisions reduce the ion beam below the detection threshold by $z=50 \mathrm{~cm}$, as a result of ion-neutral collisions. ${ }^{7}$

LIF measurements of argon ions in Chi-Kung were performed with the portable diode-laser system. On the Chi-Kung source, the laser enters through a window in the center of the flange at the end of the diffusion chamber. An alternate gas inlet on the end of the source is used as a beam dump to minimize reflections. The fluorescence radiation is collected by a set of focused optics mounted to a rectangular window on the side of the diffusion chamber. (See Figure 4.3 for a picture of the custom optics mount.) The optics mount can be tilted to select the axial position, corresponding to $z=25-45 \mathrm{~cm}$ along the axis. Based on the core size of fiber optic cable used and magnification of the optics, spatial resolution is approximately $1 \mathrm{~cm}$ along the laser beam. 


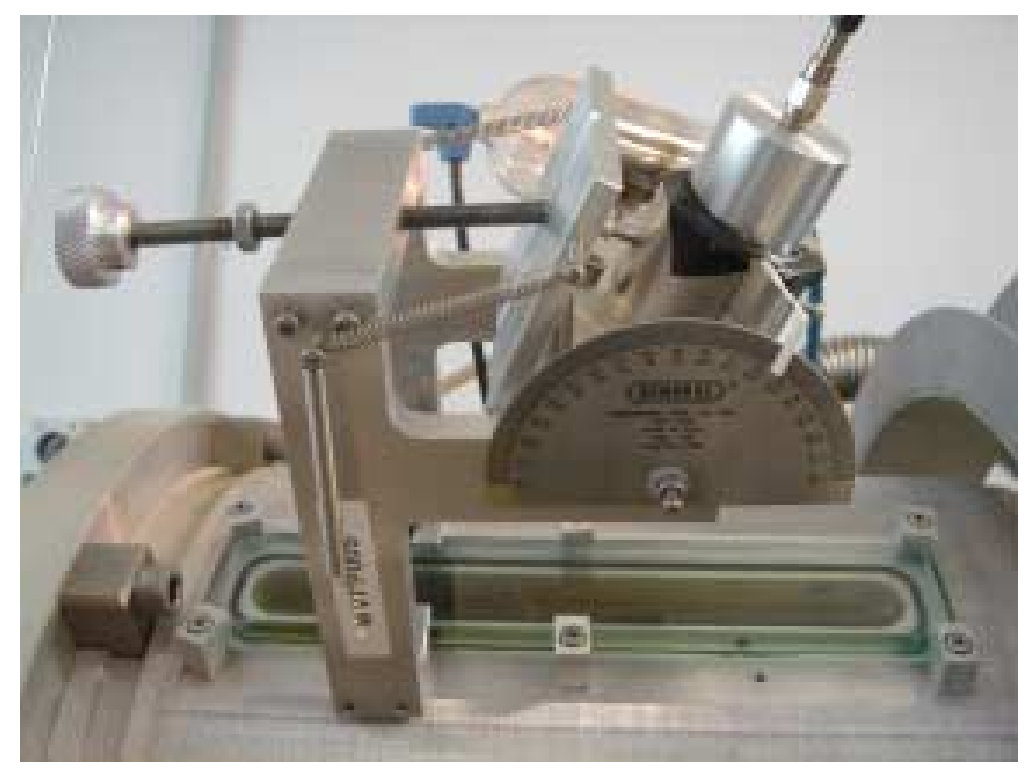

Figure 4.3 Chi-Kung tilting LIF optics mount.

Since parallel (to the magnetic field) injection is used in these experiments, only the $\sigma$ transitions are pumped. For the magnetic fields used in this experiment $(\leq 150 \mathrm{G})$ Zeeman broadening can be ignored. Thus, Equation (3.5) can be simplified such that the intensity line shape can be described by a single Doppler shifted and broadened peak:

$$
I_{R}(v)=I_{R}\left(v_{0}\right) \exp \left[\frac{-\left(v-v_{0}-\mathrm{V}_{\mathrm{o}} v_{0} / c\right)^{2}}{\alpha_{D} T_{i}}\right] .
$$

Fits of Equation 4.1 to the measured LIF intensity as a function of laser frequency yield ion temperatures and ion distribution shifts with average precisions of $\pm 0.0043 \mathrm{eV}$ and $\pm 0.27 \mathrm{GHz}$, respectively.

To provide an absolute zero velocity reference for every LIF measurement, the laser is also directed through a heated iodine cell. ${ }^{8}$ The fluorescence spectrum of the iodine cell is recorded with an amplified photodiode for each LIF laser scan and used to calculate the shift, $\Delta v$, of the central LIF peak from $\lambda_{0}=668.6138 \mathrm{~nm}$. A shift of the center of the LIF peak to lower frequency indicates a bulk flow of the ions towards the 
laser (away from the source). The parallel ion flow speed is given by $\mathrm{v}=\lambda_{0} \Delta v$. The reference spectrum used is shown in Figure 3.17.

For most measurements reported here, the molecular iodine fluorescence peak at 668.6196 $\mathrm{nm}(3.89 \mathrm{GHz}$ from the rest frame Ar II absorption line) was used as a zerovelocity reference for the LIF measurement, e.g., Figure 4.4a. For plasma conditions that yielded higher flow speeds, the molecular iodine fluorescence peak at $668.6318 \mathrm{~nm}(12.1$ $\mathrm{GHz}$ from the rest frame Ar II absorption line) was used, e.g. Figure 4.4b. The absolute wavelengths of the iodine cell peaks were measured with an accuracy of $\pm 0.0004 \mathrm{~nm}$ (according to the stated wavemeter accuracy), yielding an error in the absolute bulk ion flow speed determination of $\pm 180 \mathrm{~m} / \mathrm{s}$. This systematic error is combined with the statistical error in determining the center of LIF peak (approximately $\pm 20 \mathrm{~m} / \mathrm{s}$ on average) for the total parallel ion flow speed error shown in Figure 4.8 and Figure 4.9 (typically $\pm 181 \mathrm{~m} / \mathrm{s}$ ). If we include the overall shift in the iodine line wavelengths suggested by the recent Woo et al. measurements, the ion flow speeds reported here would decrease by approximately $900 \mathrm{~m} / \mathrm{s}$ (approximately $10 \%-20 \%$ of the measured ion beam velocities).

Because low plasma densities $\left(\sim 10^{9} \mathrm{~cm}^{-3}\right.$ in the diffusion chamber) required long lock-in integration times ( $1 \mathrm{~s})$ to differentiate background plasma emission from the laser induced fluorescence, at least five one-minute laser frequency scans were needed to obtain adequate signal-to-noise. RF powers greater than $400 \mathrm{~W}$ were required to obtain LIF signal and RF powers $>750 \mathrm{~W}$ were problematic given the need to operate the source long enough to acquire a complete LIF scan (at RF powers greater than $750 \mathrm{~W}$ the matching circuit and RF antenna would overheat during the measurement). Therefore, 
most measurements were performed at a RF power of $740 \mathrm{~W}$. Except for the magnetic field strength parameter scan, the source was operated in either the strong magnetic field case with $I_{B}=6$ Amps in each coil (Figure 4.1b) or in the weak magnetic field case with $I_{B}=3$ Amps in each coil. For all source conditions, the best signal to noise was obtained at $z=25 \mathrm{~cm}$, just inside the source.
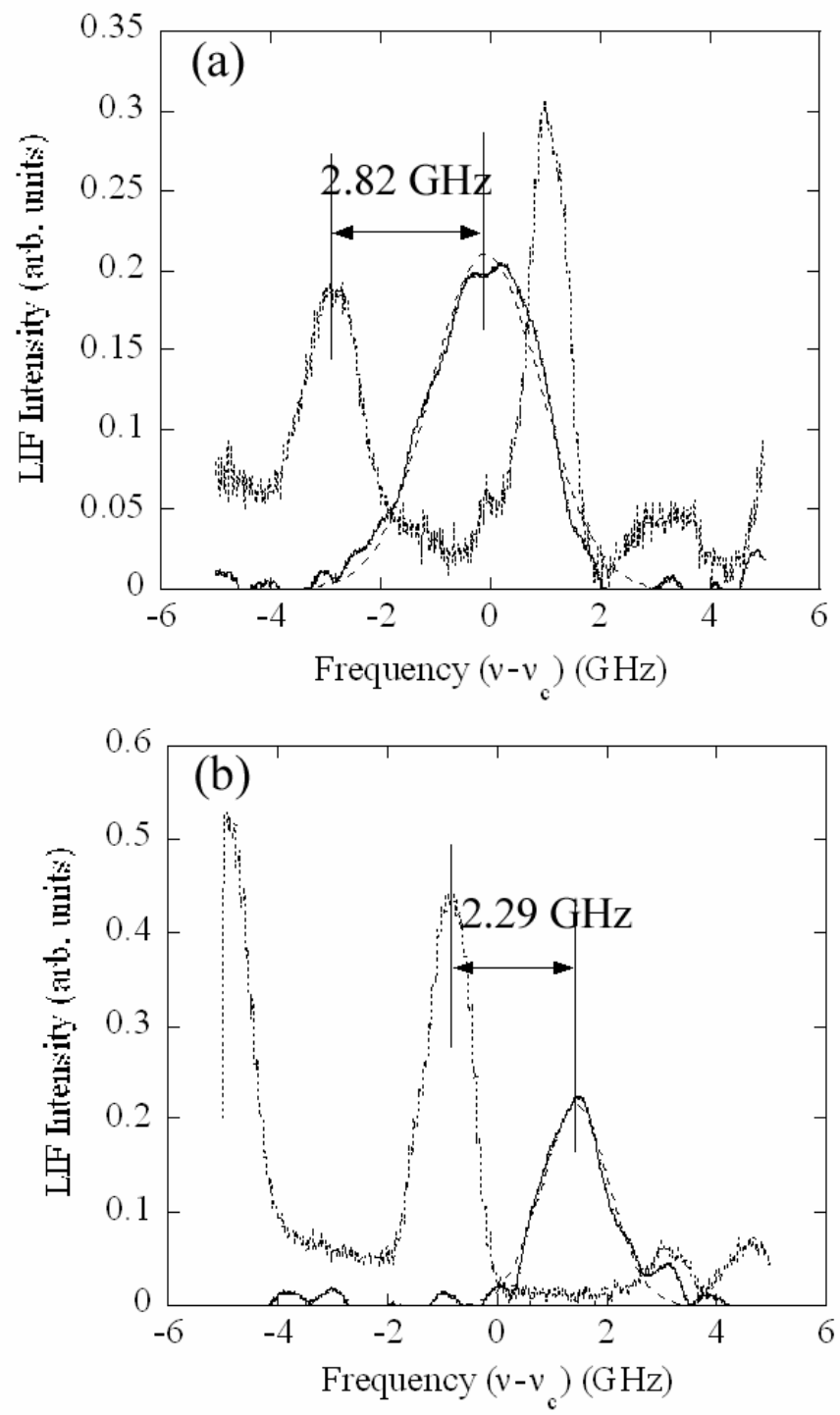

Figure 4.4 Typical LIF signal versus laser frequency (solid line) with Maxwellian fit (dashed line). Also shown is the iodine cell fluorescence spectrum for the same scan of the laser frequency (dotted line). The frequency difference between the LIF peak and the (a) $668.6196 \mathrm{~nm}$ and (b) $668.6318 \mathrm{~nm}$ iodine fluorescence peak is shown. ( $0 \mathrm{GHz}$ on the abscissa corresponds only to the center, $v_{c}$, of the laser scan window, not to zero-velocity). 


\subsection{Ion velocity distribution measurements}

The log of LIF signal versus parallel ion flow speed and axial position for a pressure of 1.3 mTorr and in low magnetic strength field case is shown in Figure 4.5a. From $z=25 \mathrm{~cm}$ to $30 \mathrm{~cm}$, the LIF measurements show an ion population accelerating from approximately $2000 \mathrm{~m} / \mathrm{s}$ to $4000 \mathrm{~m} / \mathrm{s}$. Defining the DL location as that point in the plasma where the trapped ion population appears, the LIF measurements yield a DL location of approximately $z=28 \mathrm{~cm}$ for the low magnetic field strength case. The $z=25$ cm DL location measured by Charles and Boswell (Figure 4.1c) was obtained for the strong magnetic field strength case and at significantly lower neutral pressure $(0.2$ mTorr). At similar neutral pressures $(\sim 1.0 \mathrm{mTorr})$, the RFEA probe indicates an ion beam energy of $8 \mathrm{eV}$ (corresponding to $\sim 6200 \mathrm{~m} / \mathrm{s}$ ) and a beam density of a few $10^{9} \mathrm{~cm}^{-3}$. Since there was a chance that the RFEA probe, with its conducting, grounded shaft, could perturb the plasma enough to inadvertently create the DL structure, LIF measurements were taken with and without a mock RFEA inserted in the plasma. The LIF measurements were unaffected by the presence of the probe located $12.6 \mathrm{~cm}$ away from the LIF interrogation volume. 


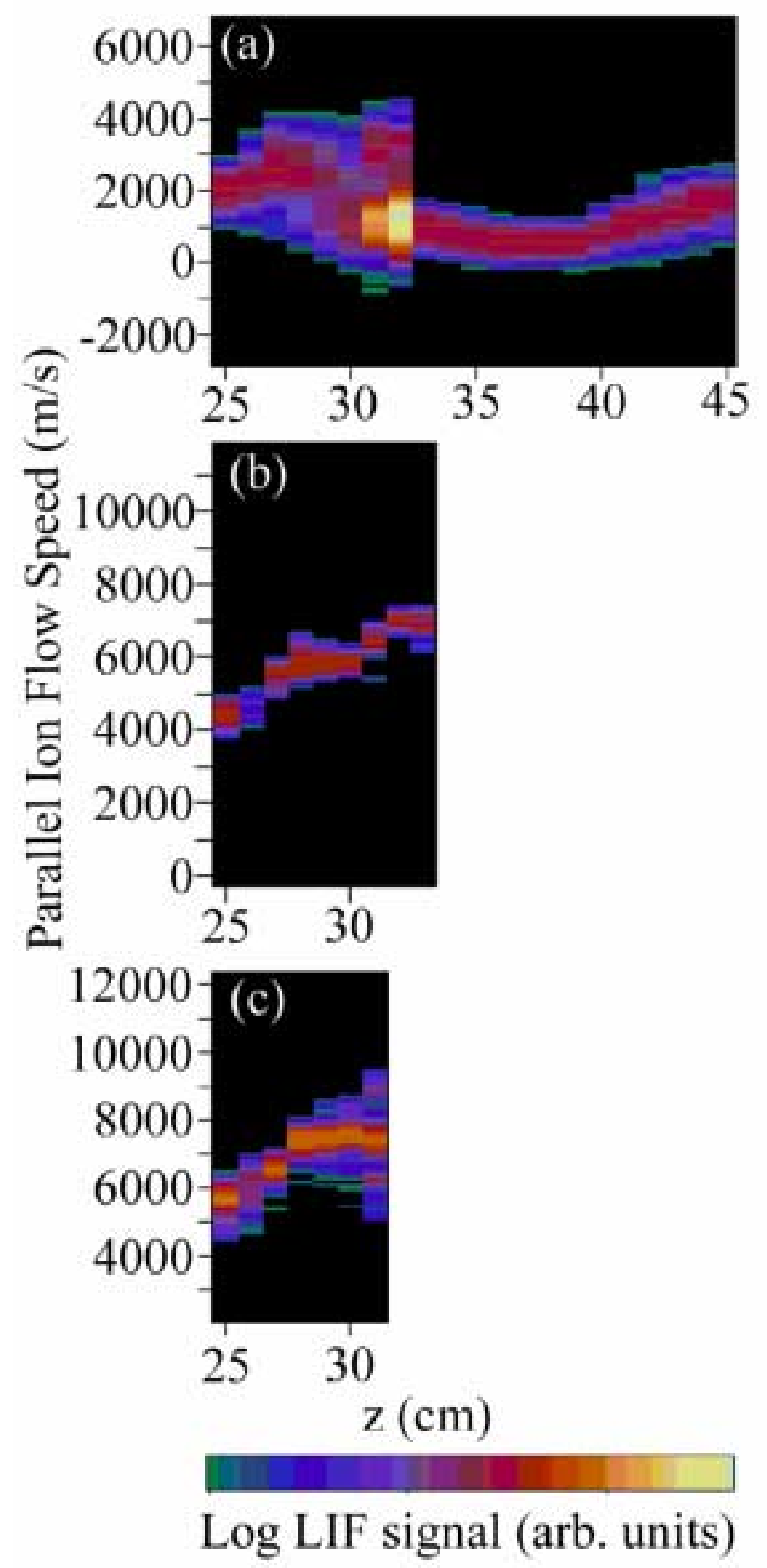

Figure 4.5 LIF signal versus parallel argon ion flow speed (away from the source) and axial position for (a) $P=1.3 \mathrm{mTorr}$, weak magnetic field case, (b) $P=0.55 \mathrm{mTorr}$, strong magnetic field case, and (c) $P=0.37$ mTorr, strong magnetic field case. RF power is $740 \mathrm{~W}$ in all cases. 
For lower neutral pressures $(<1$ mTorr $)$ and for the strong magnetic field case, useful LIF signal was only achieved between axial positions $z=25-32 \mathrm{~cm}$. Below neutral pressures of 0.35 mTorr, LIF measurements were not possible anywhere along the axis of the experiment - most likely due to significantly reduced plasma densities downstream of the DL at low neutral pressure.

Figure $4.5 \mathrm{~b}$ and Figure $4.5 \mathrm{c}$ show the log of LIF signal versus parallel ion flow speed and axial position for neutral pressures of 0.55 mTorr and 0.37 mTorr, respectively. Note that the ion flow speed upstream of the DL increases as the pressure decreases, indicating a larger potential difference across the DL for lower pressures. For $P=0.55$ mTorr and $P=0.37$ mTorr, the maximum ion speeds measured by LIF were approximately $7000 \mathrm{~m} / \mathrm{s}$ and $7300 \mathrm{~m} / \mathrm{s}$ (corresponding to ion beam energies of $10.1 \mathrm{eV}$ and $11.0 \mathrm{eV}$ ), respectively. Based on the measured downstream electron temperatures of $7 \mathrm{eV}$ and $8 \mathrm{eV}$, respectively, the ion flow speeds are clearly supersonic compared to ion sound speeds of $5300 \mathrm{~m} / \mathrm{s}$ for $0.55 \mathrm{mTorr}$ and $5700 \mathrm{~m} / \mathrm{s}$ for $0.33 \mathrm{mTorr}$. Since the LIF signal was lost near the DL, the final ion flow speeds could not be measured with LIF. We expect that additional ion acceleration up to similar energies as measured by the RFEA occurred in the DL - which would be consistent with the RFEA probe measured ion beam energies downstream of the DL at $z=37 \mathrm{~cm}: 13.4 \mathrm{eV}$ at $0.55 \mathrm{mTorr}$ (obtained at a RF power of $250 \mathrm{~W}$ and not the $740 \mathrm{~W}$ used here) and $15.2 \mathrm{eV}$ at 0.33 mTorr. These spatially resolved LIF measurements of the ion velocity distribution function (IVDF) also show that as the neutral pressure decreases, the DL forms further inside the diffusion chamber (based on the ion beam acceleration continuing further downstream for higher pressures). 
The LIF intensity of the ion beam population measured in Figure 4.5a decreases rapidly with distance downstream of the DL. The amplitude of the LIF signal for the ion beam population is shown as a function of distance downstream of the DL in Figure 4.6. An exponential fit to the measurements yields a 1/e folding distance of $3.6 \mathrm{~cm}$. Assuming that the decrease is due to a rapidly decreasing population of metastable ions in the beam and the rapidly expanding magnetic field (decreasing plasma density), the 1/e folding distance provides a measure of the quenching rate of the metastable ions. The 1/e folding distance for the ion beam signal obtained in these measurements is inconsistent with what was observed in MNX (1.6 cm for a pressure of 0.135 mTorr $)^{2}$ and other argon LIF experiments. ${ }^{9}$ Using the $1 / \mathrm{e}$ folding distance obtained by Cohen et al., ${ }^{2}$ one would expect to find a $1 / \mathrm{e}$ folding distance of $0.4 \mathrm{~cm}$ for a pressure of $1.3 \mathrm{mTorr}$ in Chi-Kung, assuming the same collisional quenching-cross-section for the metastable state. In other words, the LIF signal persists much further downstream of the DL in Chi-Kung then expected. The very low pressure MNX experimental configuration included a physical aperture that limited plasma flow and therefore plasma production downstream of the DL. The Chi-Kung experiment has no aperture and we hypothesize that the longer 1/e folding distance results from additional metastable production by high energy electrons throughout the measurement region in this higher pressure plasma. As noted above, at lower neutral pressures the LIF signal decreases much more rapidly downstream of the DL. 


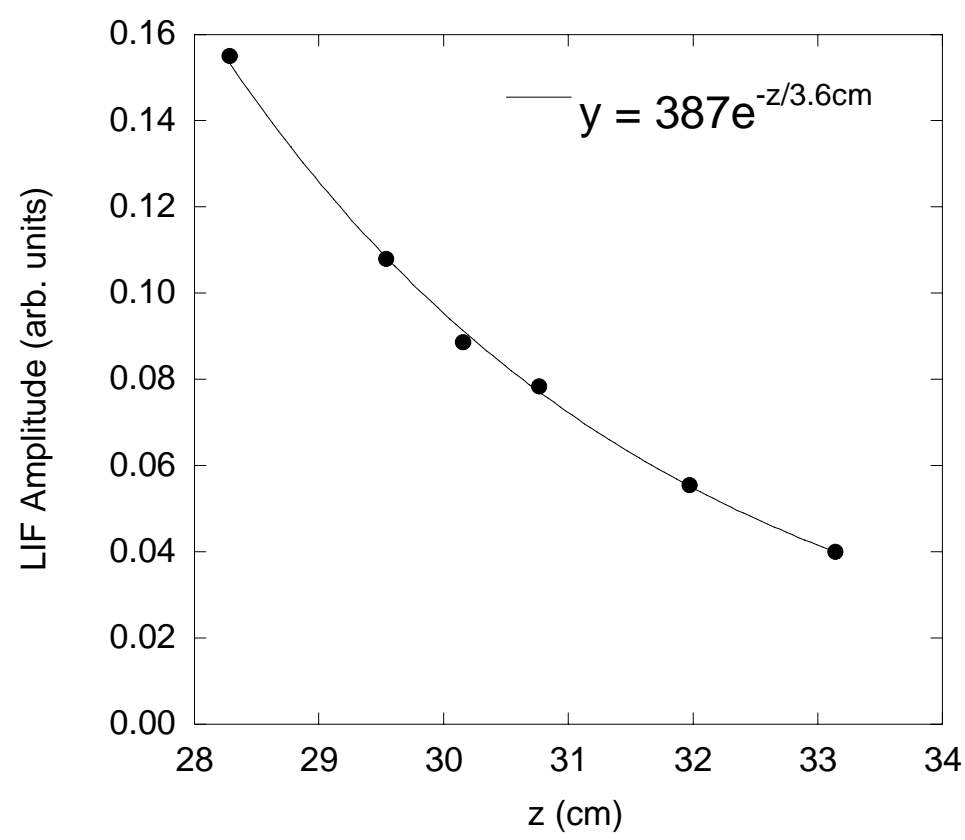

Figure 4.6 LIF signal amplitude of ion beam and exponential fit versus axial position from data of Figure 4.5a.

Assuming energy conservation, changes in the measured ion beam energy can provide a measurement of the local gradient in the plasma potential. The spatial profile of the plasma potential, as estimated from the ion beam energy, is shown in Figure 4.7 for the LIF data of Figure 4.5a and Figure 4.5b. Also shown, for comparison, is the plasma potential measured by RFEA at $0.2 \mathrm{mTorr}$ (Figure $4.2 \mathrm{c}$ ). It can be seen that the plasma potential in the pre-sheath of the DL has a steeper gradient at lower pressures. Also, the location of the DL appears to move further downstream of the source as the pressure increases. 


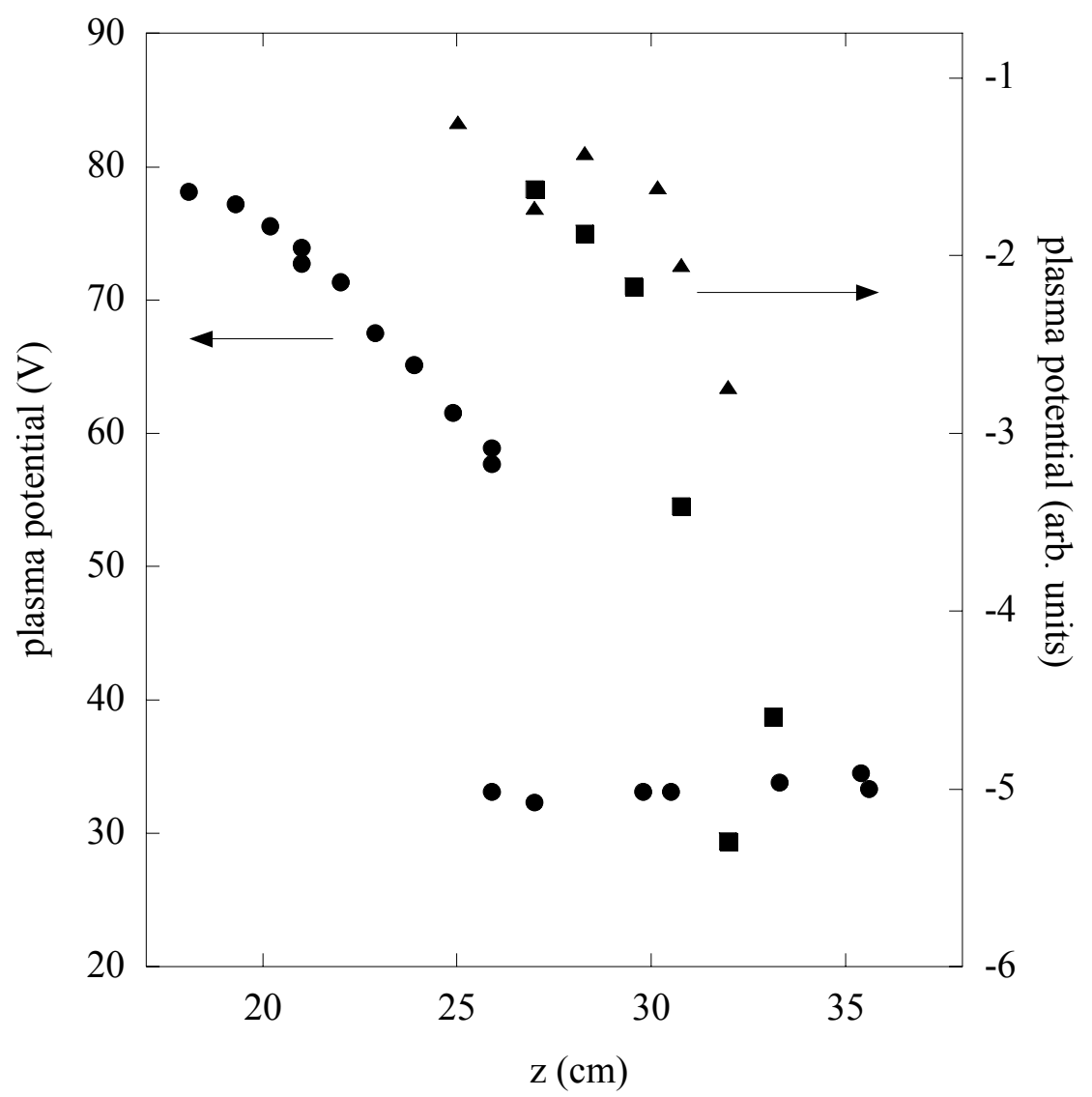

Figure 4.7 Plasma potential estimated from ion beam energy versus position from data of Figure 4.5a (filled triangles) and Figure $4.5 \mathrm{~b}$ (filled squares). Plasma potential versus position measured by RFEA at 0.2 mTorr (open circles). ${ }^{6}$

A pressure scan at $z=25 \mathrm{~cm}$ (just upstream of the DL), RF power $=740 \mathrm{~W}$, and for the strong magnetic field case is shown in Figure 4.8. As shown in Figure 4.5, there is a substantial increase in the parallel ion flow speed into the double layer with decreasing pressure. A similar scaling of ion flow speed on inverse neutral pressure was reported by Sun et al. in HELIX/LEIA, which is a higher density, expanding helicon source plasma. ${ }^{10}$ At the lowest neutral pressure, the ion flow speed reaches $6000 \mathrm{~m} / \mathrm{s}$ well upstream of the DL. As can be seen in Figure 4.5c, at the lowest neutral pressure of 0.37 mTorr the parallel ion speed reaches nearly $8000 \mathrm{~m} / \mathrm{s}$ at the furthest downstream location with 
useful LIF emission. That the ion beam energy scales as $1 / P_{o}{ }^{2}$ with the neutral pressure, suggests that the double layer strength also scales with $1 / P_{o}^{2}$.

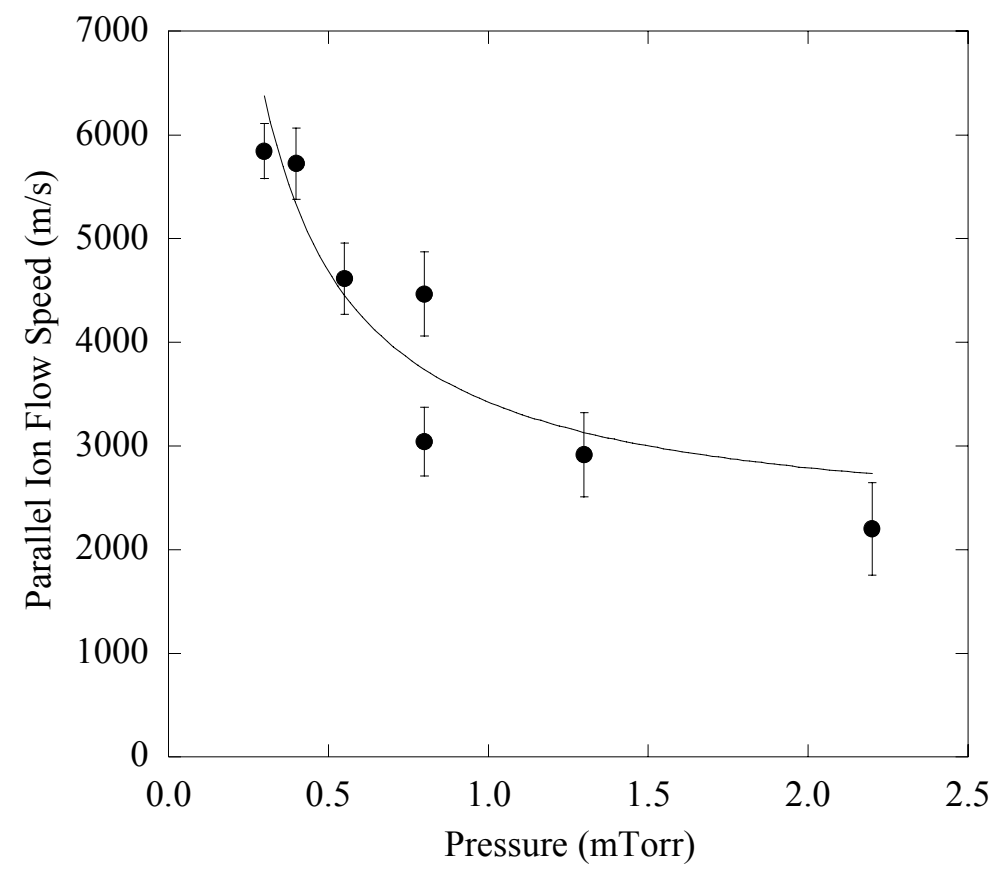

Figure 4.8 Parallel ion flow speed versus neutral pressure at $z=25 \mathrm{~cm}, \mathrm{RF}$ power $=740 \mathrm{~W}$, and for the strong magnetic field case. The solid line is given by $\mathrm{v}_{i o}=\left(1267 / P_{o}+2156\right) \mathrm{m} / \mathrm{s}$.

In Figure 4.9a, the parallel ion flow speed is shown as a function of magnetic field strength for the same current in both coils. In Figure 4.9b, the ion flow speed measurements are for the case where the top coil (furthest from the diffusion chamber) was held at 6 Amps while the current through the bottom coil was varied. Note that a current of 6 Amps in both coils yields the strong magnetic field case geometry shown in Figure 4.2b. Because Figure 4.9a indicates that as the magnetic field strength increases the ions flow into the diffusion chamber faster, it would be natural to assume that the increased ion flow arises from magnetic moment conservation leading to increased conversion of perpendicular thermal energy into directed parallel ion flow. However, the measurements shown in Figure $4.9 \mathrm{~b}$ demonstrate that it is the coil furthest from the 
diffusion chamber that has the most influence over the ion flow speed into the diffusion chamber. Thus, the strength of the double layer is controlled by the plasma source parameters (i.e., the magnetic field strength in the source), and the high speed ion flows result from acceleration in the spontaneously formed DL and not any magnetic moment conservation process in the diverging magnetic field. The dependence of the potential drop across the DL on the coil furthest from the diffusion chamber is confirmed by RFEA measurements. $^{11}$
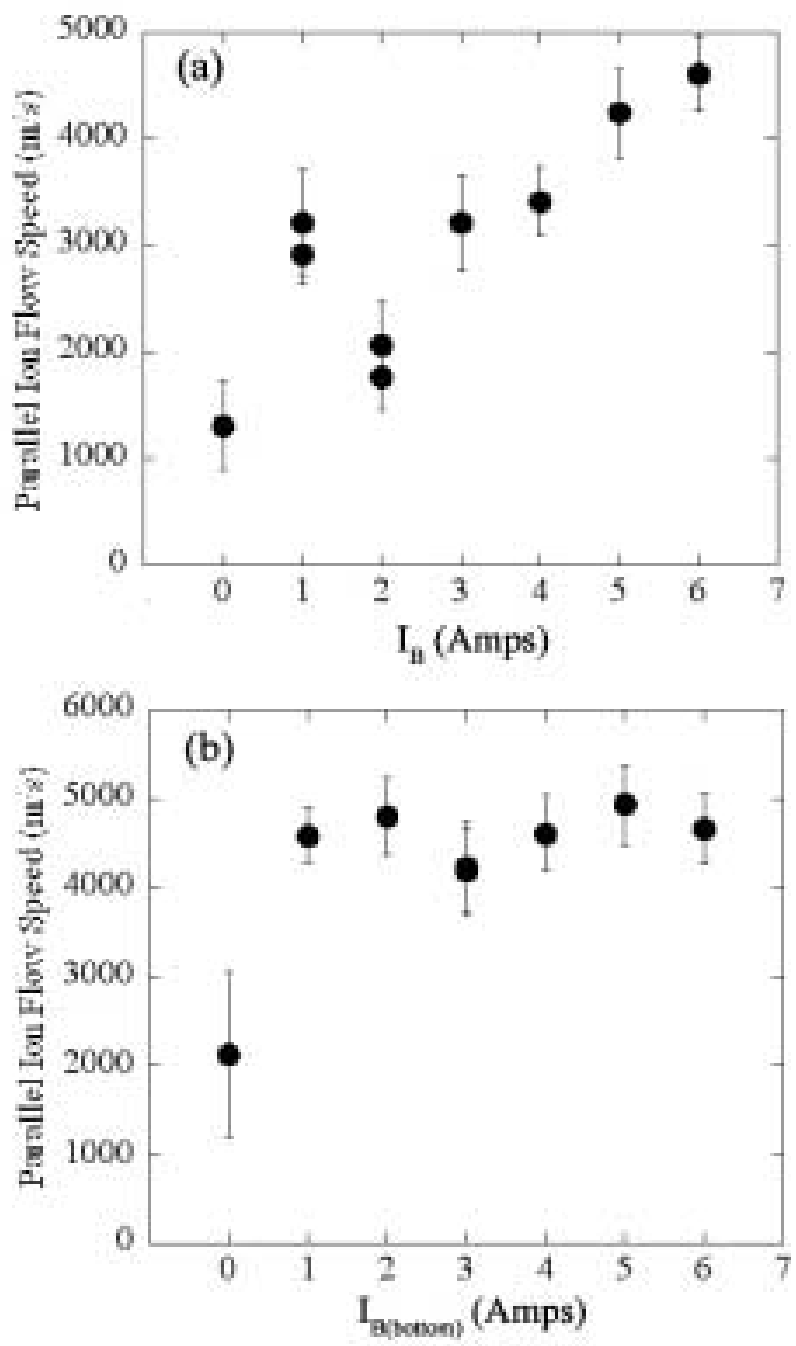

Figure 4.9 Parallel ion flow speed versus magnet coil current (magnetic field) for (a) equal currents in both coils and (b) top coil held at 6 Amps while current through bottom coil was varied. RF power of $740 \mathrm{~W}, P=0.55 \mathrm{mTorr}$, and $z=25 \mathrm{~cm}$. 


\subsection{Discussion}

The high spatial and velocity resolution of the LIF IVDF measurements shown in Figure 4.5 provide a unique opportunity for comparison with numerical predictions of the structure and magnitude of DL formation in expanding, current-free plasmas. A recently developed one-dimensional Monte-Carlo Collision ${ }^{12}$ Particle-in-Cell ${ }^{13}$ (MCC-PIC) plasma computer code was used to confirm that DLs could form in current-free expanding plasmas. ${ }^{14}$ The PIC simulation consisted of a bounded plasma with a floating left wall and a grounded right wall. The system was separated into two regions: the source region and the diffusion chamber. In the source region, the electrons are heated up by a uniform RF electric field of $10 \mathrm{MHz}$ perpendicular to the axis of the simulation. In the diffusion chamber, the diffusion of the plasma in the diverging magnetic field was modeled with a loss mechanism (see Figure 4.10).

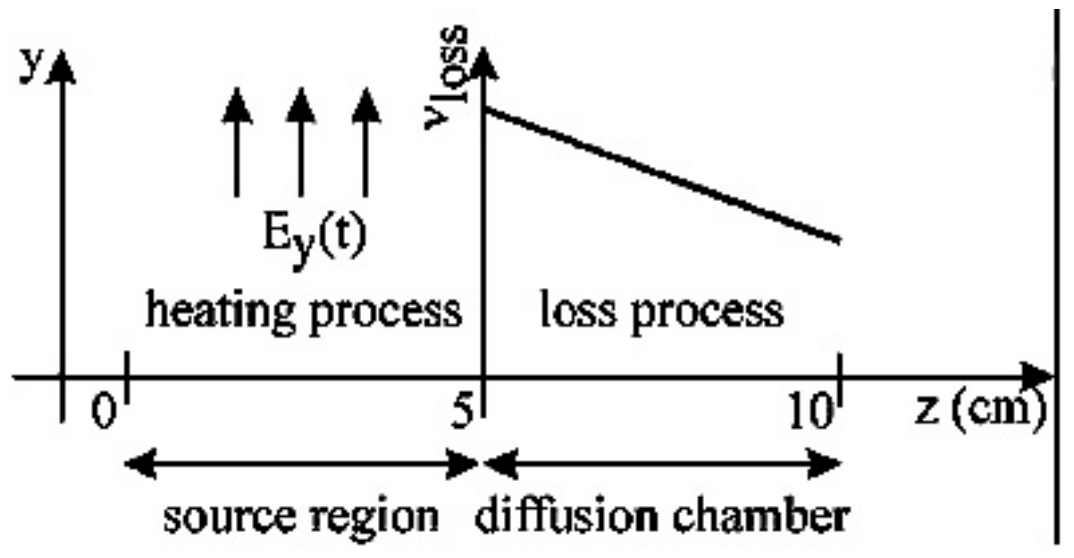

Figure 4.10 Spatial dependence of electron heating and loss rate used in PIC model of plasma expansion.

The ion velocity distribution in phase-space, where the abscissa represents the position and the ordinate the ion velocity, predicted by the PIC code is shown in Figure 4.11 for a simulation neutral pressure of $1 \mathrm{mTorr}$. Throughout the simulation, a low energy population of ions is observed which corresponds to the ions which are created by 
ionization and charge exchange collisions. Downstream of the DL a high energy population can be seen which corresponds to the ions accelerated while traversing the potential drop of the DL. Note that the acceleration of the ions occurs over many centimeters in the simulation (in the pre-sheath and the sheath) while the actual DL is much narrower and appears in the ion phase space plot as a narrow region of strong ion acceleration. The acceleration of the background ion population to the boundaries of the simulation volume (as the ions fall through the sheath) is also evident at the left and right sides of Figure 4.11. The spatial structure, beam energy, character of ion acceleration region, and ion heating in the pre-sheath in the simulation are all consistent with the LIF measurements shown in Figure 4.5a.

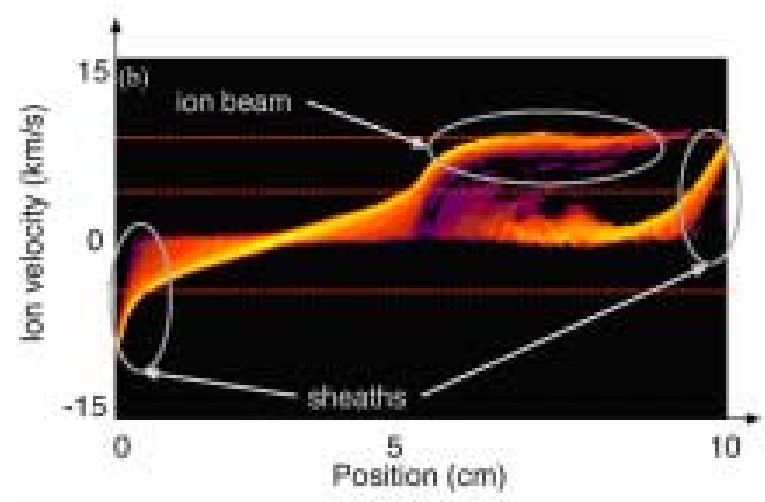

Figure 4.11 Parallel ion velocity distribution function along MCC-PIC simulation axis. ${ }^{14}$

The LIF measurements show acceleration of ions into the double layer, as well as a fast ion beam and slow trapped population downstream of the double layer. Even if the Woo et al. iodine line wavelength measurements are accurate, the overall trend would be the same, with only the magnitudes of the velocities decreased by $10-20 \%$. The distribution of the slow trapped population would shift to a bulk velocity of zero (see Figure 4.5a), consistent with expectations for the trapped ions. We found that the 
potential drop of the DL in Chi-Kung increases with decreasing pressure and that the LIF ion beam energy measurements agree with previous RFEA measurements. The LIF measurements also demonstrated that the presence of the RFEA probe did not significantly affect the magnitude of the observed ion beam energy. Perhaps most significantly, the dependence of the ion beam energy on the upstream plasma conditions and not the details of the magnetic field structure in the expansion region suggest that optimal ion acceleration can be achieved through optimization of the upstream plasma parameters. Any future LIF measurements in Chi-Kung should investigate the off-axis parallel ion flow to better understand the radial distribution of the ions and also include argon neutral LIF measurements to investigate the possibility of momentum coupling to the neutrals (plasma detachment) through charge-exchange collisions with ions. 


\section{References}

${ }^{1}$ C. Charles and R.W. Boswell, Phys. Plasmas 11 (2004) 1706.

${ }^{2}$ S. A. Cohen, N.S. Siefert, S. Stange, E.E. Scime, R. F. Boivin, and F. Levinton, Phys. Plasmas 10, 2593 (2003).

${ }^{3}$ X. Sun, A. Keesee, C. Biloiu, E. Scime, C. Charles, R. Boswell, and A. Meige, Phys. Rev. Lett. 95, 025004 (2005).

${ }^{4}$ A. Meige, R. Boswell, C. Charles, J.-P. Boeuf, G. Hagelaar, and M. Turner. IEEE Trans. Plasma Sci. 33, 334 (2005).

${ }^{5}$ K.K. Chi, T.E. Sheridan, and R.W Boswell, Plasma Sources Sci. Technol. 8, 421 (1999).

${ }^{6}$ C. Charles and R.W. Boswell, Appl. Phys. Lett. 82,1356 (2003).

${ }^{7}$ C. Charles and R.W. Boswell, Phys. Plasmas 11, 3808 (2004).

${ }^{8}$ A.M. Keesee, E.E. Scime, and R.F. Boivin, Rev. Sci. Instrum. 75, 4091(2004).

${ }^{9}$ F. Skiff, G. Bachet, and F. Doveil, Phys. Plasmas 8, 3139 (2001).

${ }^{10}$ X. Sun, C. Biloiu, R. Hardin, and E. Scime, Plasma Sources Sci. Technol. 13, 359 (2004).

${ }^{11}$ C. Charles, Phys. Plasmas 12, 044508 (2005).

${ }^{12}$ V. Vahedi and M. Surendra, Comp. Phys. Comm. 87, 179 (1995).

${ }^{13}$ C. K. Birdsall and D. Fuss, J. Comp. Phys. 3, 494 (1969).

${ }^{14}$ A. Meige, R.W. Boswell, C. Charles, and M. Turner, Phys. Plasmas 12, 052317 (2005). 


\section{Chapter 5: Collisional-Radiative Modeling}

When using diagnostics such as LIF and spectroscopy, the parameters measured are characteristic of the probed atomic or ionic state. However, we are typically interested in knowing these characteristics for the overall population. For neutral argon parameters such as temperature and flow velocity, we expect the values to be the same for all states. Density, on the other hand, depends strongly on the atomic state investigated. We estimate the total density by the density of the atomic ground state because $N(1) \approx N$, where $N(1)$ is the ground state density and $N$ is the total density (i.e. the density of all excited states together is much smaller than that of the ground state density for low temperature plasmas). For LIF, the "pump state" is the state that is probed. In passive emission spectroscopy, it is the initial (upper) state. Specifically, we are studying the $4 \mathrm{~s}[3 / 2]^{\circ}{ }_{1}$ state with LIF and the $4 \mathrm{p}^{\prime}[1 / 2]_{0}$ state with emission spectroscopy. To obtain the ground state density from the state density measured, we must know how the two state densities are related to the atomic ground state.

There are two basic models that describe the relationship of atomic state densities in a plasma. The Steady State Corona (SSC) model takes into account electron impact excitation from the ground state to a given state and spontaneous de-excitation from that state to lower states. The density of the $i^{\text {th }}$ atomic state is given by

$$
N_{i}=\frac{n_{e} N_{o}\langle\sigma v\rangle_{o i}}{\sum_{j<i} A_{i j}},
$$

where $n_{e}$ is the electron density, $\langle\sigma v\rangle_{o i}$ is the electron impact excitation rate coefficient from the ground to the $i^{\text {th }}$ state, and $A_{i j}$ is the spontaneous decay rate (transition probability) from the $i^{\text {th }}$ state to the $j^{\text {th }}$ state. A more complete description of the state 
densities is given by the Collisional-Radiative (CR) model, which relates the density of each state based on collisional excitation and de-excitation from all states, spontaneous de-excitation, and ionization. The CR model is a set of differential equations of the form such as

$$
\frac{d N_{i}}{d t}=n_{e} \sum_{j \neq i} S_{j i} N_{j}+\sum_{j>i} A_{j i} N_{j}-n_{e} \sum_{j \neq i} S_{i j} N_{i}-\sum_{j<i} A_{i j} N_{i}-n_{e} I_{i} N_{i}=0,
$$

where $S_{j i}$ is the collisional coefficient from the $j^{\text {th }}$ to the $i^{\text {th }}$ state (given by $\langle\sigma v\rangle_{j i}$ ), $A_{j i}$ is the spontaneous de-excitation coefficient from the $j^{\text {th }}$ to the $i^{\text {th }}$ state, and $I_{i}$ is the ionization coefficient for the $i^{\text {th }}$ state. Thus, this equation describes, from left to right, the collisional excitation and de-excitation into state $i$, the spontaneous de-excitation into state $i$, the collisional excitation and de-excitation out of state $i$, the spontaneous deexcitation out of state $i$, and the ionization out of state $i$. Some CR models include additional terms to describe other processes, depending on the system which they are describing.

Some groups have used the SSC model when describing helicon source dynamics. ${ }^{1,2}$ The SSC model is accurate for densities up to $10^{11} \mathrm{~cm}^{-3}$, making it useful for low density hydrogen and helium helicon plasmas. ${ }^{3}$ For higher densities, however, it is important to take into account the additional processes as described using the CR model. In addition, argon is inherently complex because it has 18 electrons, has several low energy metastable states that play a role in stepwise excitation, and has a low ionization energy threshold. ${ }^{4,5}$ Therefore, it is important to use a CR model for argon plasmas even at low densities and temperatures.

Because a CR model includes interactions between all (or as many as can be described) of the energy levels, an equation of the form of Equation (5.2) is needed for 
each of these levels, leading to a large set of coupled differential equations. Additionally, the coefficients of all the terms in Equation (5.2) must be known for the atom of interest, which is not always the case. These factors make CR models quite complicated. CR models have been developed for hydrogen, hydrogen-like ions, helium, argon, and argon ions. ${ }^{6}$

Many CR models assume a Maxwellian electron energy distribution function (EEDF). Because many plasmas studied have non-Maxwellian EEDFs, Vlček developed a CR model for argon that allows input of an arbitrary EEDF. ${ }^{7}$ This CR model includes sixty-five effective energy levels of neutral argon. Vlček also included atom-atom inelastic collisions and diffusion losses in the CR model. Bogaerts et al. modified this CR model to include more details of the two $4 \mathrm{~s}$ metastable levels and fast argon ion and atom impact ionization and excitation. ${ }^{8}$ The latter was included to better describe the "cathode dark space" of the DC glow discharge studied. The Bogaerts CR model was selected for this project in order to take advantage of the ability to input a spatially dependent EEDF. We then modified the model to include spatial dependence of the argon neutral ground state. See Appendix D for the Matlab ${ }^{\circledR}$ code used to create the EEDFs and the FORTRAN code used for the CR model.

The effective levels used in the CR model code are given in Table 5.1, numbered in order of their ionization energies. ${ }^{7,8}$ Also included in the table is the excitation energy in $\mathrm{eV}$ for electron-impact excitation from the ground state, the statistical weight (number of states of that energy, $g_{\mathrm{n}}=2 J+1$ ), and whether the transition is optically allowed, parityforbidden, or spin-forbidden for each level. The argon energy levels can be divided into two subgroups, one in which the parent ion has a quantum number $j=1 / 2$ denoted by a 
prime, and the other in which the parent ion has a quantum number $j=3 / 2$. A diagram of the argon energy levels divided into the two subsystems is shown in Figure 5.1 with the effective level numbers used in the CR model.

Table 5.1 The 65 effective argon levels included in the CR model code and properties associated with excitation by electrons from the ground state to each level. A is an optically allowed, $\mathrm{P}$ is a parity-forbidden, and $\mathrm{S}$ is a spin-forbidden transition.

\begin{tabular}{|c|c|c|c|c|}
\hline $\begin{array}{l}\text { Effective level } \\
\text { number, } n\end{array}$ & Designation $\mathrm{n}_{\mathrm{pgn}}[\mathrm{K}]_{\mathrm{J}}$ & Excitation energy (eV) & $\begin{array}{l}\text { Statistical } \\
\text { weight, } g_{n}\end{array}$ & $\begin{array}{l}\text { Nature of } \\
\text { transition }\end{array}$ \\
\hline 1 & $3 p^{6} s$ & 0.000 & -5 & - \\
\hline 2 & $4 \mathrm{~s}[3 / 2]_{2}$ & 11.548 & 5 & $\mathrm{~s}$ \\
\hline 3 & $4 s[3 / 2]_{1}$ & 11.624 & 3 & A \\
\hline 4 & $4 \mathrm{~s}^{\prime}[1 / 2]_{0}$ & 11.723 & 1 & $\mathrm{~s}$ \\
\hline 5 & $4 s^{\prime}[1 / 2]_{1}$ & 11.828 & 3 & A \\
\hline 6 & $4 p[1 / 2]_{1}$ & 12.907 & 3 & $\mathrm{P}$ \\
\hline 7 & $4 \mathrm{p}[3 / 2]_{1,2}+[5 / 2]_{2,3}$ & 13.116 & 20 & $\mathrm{P}$ \\
\hline 8 & $4 p^{\prime}[3 / 2]_{1,2}$ & 13.295 & 8 & $\mathrm{P}$ \\
\hline 9 & $4 p^{\prime}[1 / 2]_{1}$ & 13.328 & 3 & $\mathrm{P}$ \\
\hline 10 & $4 \mathrm{p}[1 / 2]_{0}$ & 13.271 & 1 & $\mathrm{P}$ \\
\hline 11 & $4 p^{\prime}[1 / 2]_{0}$ & 13.480 & 1 & $\mathrm{P}$ \\
\hline 12 & $3 \mathrm{~d}[1 / 2]_{0,1}+[3 / 2]_{2}$ & 13.884 & 9 & $\mathrm{~S}$ \\
\hline 13 & $3 \mathrm{~d}[7 / 2]_{3,4}$ & 13.994 & 16 & $\mathrm{~s}$ \\
\hline 14 & $3 d^{\prime}[3 / 2]_{2}+[5 / 2]_{2,3}$ & 14.229 & 17 & $\mathrm{P}$ \\
\hline 15 & $5 s^{\prime}$ & 14.252 & 4 & A \\
\hline 16 & $3 \mathrm{~d}[3 / 2]_{1}+[5 / 2]_{2,3}+5 \mathrm{~s}$ & 14.090 & 23 & A \\
\hline 17 & $3 d^{\prime}[3 / 2]_{1}$ & 14.304 & 3 & A \\
\hline 18 & $5 p$ & 14.509 & 24 & $\mathrm{P}$ \\
\hline 19 & $5 p^{\prime}$ & 14.690 & 12 & $\mathrm{P}$ \\
\hline 20 & $4 d+6 s$ & 14.792 & 48 & A \\
\hline 21 & $4 d^{\prime}+6 s^{\prime}$ & 14.976 & 24 & A \\
\hline 22 & $4 f^{\prime}$ & 15.083 & 28 & - \\
\hline 23 & $4 f$ & 14.906 & 56 & - \\
\hline 24 & $6 p^{\prime}$ & 15.205 & 12 & - \\
\hline 25 & $6 \mathrm{p}$ & 15.028 & 24 & - \\
\hline 26 & $5 d^{\prime}+7 s^{\prime}$ & 15.324 & 24 & A \\
\hline 27 & $5 d+7 s$ & 15.153 & 48 & A \\
\hline 28 & $5 f^{\prime}, g^{\prime}$ & 15.393 & 64 & - \\
\hline 29 & $5 f, g$ & 15.215 & 128 & - \\
\hline 30 & $7 p^{\prime}$ & 15.461 & 12 & - \\
\hline 31 & $7 p$ & 15.282 & 24 & - \\
\hline 32 & $6 d^{\prime}+8 s^{\prime}$ & 15.520 & 24 & - \\
\hline 33 & $6 \mathrm{~d}+8 \mathrm{~s}$ & 15.347 & 48 & A \\
\hline 34 & $6 f^{\prime}, g^{\prime}, h^{\prime}$ & 15.560 & 108 & - \\
\hline 35 & $6 f, g, h$ & 15.382 & 216 & - \\
\hline 36 & $8 p^{\prime}$ & 15.600 & 12 & - \\
\hline 37 & $8 p$ & 15.423 & 24 & - \\
\hline 38 & $7 d^{\prime}+9 s^{\prime}$ & 15.636 & 24 & - \\
\hline 39 & $7 d+9 s$ & 15.460 & 48 & - \\
\hline 40 & $7 f^{\prime}, g^{\prime}, h^{\prime}, i^{\prime}$ & 15.659 & 460 & - \\
\hline 41 & $7 f, g, h, i$ & 15.482 & 320 & - \\
\hline 42 & $8 d^{\prime}, f^{\prime}, \ldots$ & 15.725 & 240 & - \\
\hline 43 & $8 d, f, \ldots$ & 15.548 & 480 & - \\
\hline 44 & $9 p^{\prime}, d^{\prime}, f^{\prime}, \ldots$ & 15.769 & 320 & - \\
\hline 45 & $9 p, d, f, .$. & 15.592 & 640 & - \\
\hline 46 & $10 s^{\prime}, p^{\prime}, d^{\prime}, f^{\prime}, \ldots$ & 15.801 & 400 & - \\
\hline 47 & $10 \mathrm{~s}, \mathrm{p}, \mathrm{d}, \mathrm{f}, \ldots$ & 15.624 & 800 & - \\
\hline 48 & $11 s^{\prime}, p^{\prime}, d^{\prime}, f^{\prime}, \ldots$ & 15.825 & 484 & - \\
\hline 49 & $11 \mathrm{~s}, \mathrm{p}, \mathrm{d}, \mathrm{f}, \ldots$ & 15.648 & 968 & - \\
\hline 50 & $12 s^{\prime}, p^{\prime}, d^{\prime}, f^{\prime}, \ldots$ & 15.843 & 576 & - \\
\hline 51 & $12 \mathrm{~s}, \mathrm{p}, \mathrm{d}, \mathrm{f}, \ldots$ & 15.666 & 1152 & - \\
\hline 52 & $13 s^{\prime}, p^{\prime}, d^{\prime}, f^{\prime}, \ldots$ & 15.857 & 676 & - \\
\hline 53 & $13 s, p, d, f, \ldots$ & 15.680 & 1352 & - \\
\hline 54 & $14 s^{\prime}, p^{\prime}, d^{\prime}, f^{\prime}, \ldots$ & 15.868 & 784 & - \\
\hline 55 & $14 s, p, d, f, \ldots$ & 15.691 & 1568 & - \\
\hline 56 & $15 s^{\prime}, p^{\prime}, d^{\prime}, f^{\prime}, \ldots$ & 15.877 & 900 & - \\
\hline 57 & $15 \mathrm{~s}, \mathrm{p}, \mathrm{d}, \mathrm{f}, \ldots$ & 15.700 & 1800 & - \\
\hline 58 & $16 s^{\prime}, p^{\prime}, d^{\prime}, f^{\prime}, \ldots$ & 15.884 & 1024 & - \\
\hline 59 & $16 s, p, d, f, \ldots$ & 15.707 & 2048 & - \\
\hline 60 & $17 s^{\prime}, p^{\prime}, d^{\prime}, f^{\prime}, \ldots$ & 15.890 & 1156 & - \\
\hline 61 & $17 \mathrm{~s}, \mathrm{p}, \mathrm{d}, \mathrm{f}, \ldots$ & 15.713 & 2312 & - \\
\hline 62 & $18 s^{\prime}, p^{\prime}, d^{\prime}, f^{\prime}, \ldots$ & 15.895 & 1296 & - \\
\hline 63 & $18 \mathrm{~s}, \mathrm{p}, \mathrm{d}, \mathrm{f}, \ldots$ & 15.718 & 2592 & - \\
\hline 64 & $19 s^{\prime}, p^{\prime}, d^{\prime}, f^{\prime}, \ldots$ & 15.899 & 1444 & - \\
\hline 65 & $19 \mathrm{~s}, \mathrm{p}, \mathrm{d}, \mathrm{f}, \ldots$ & 15.722 & 2888 & - \\
\hline
\end{tabular}




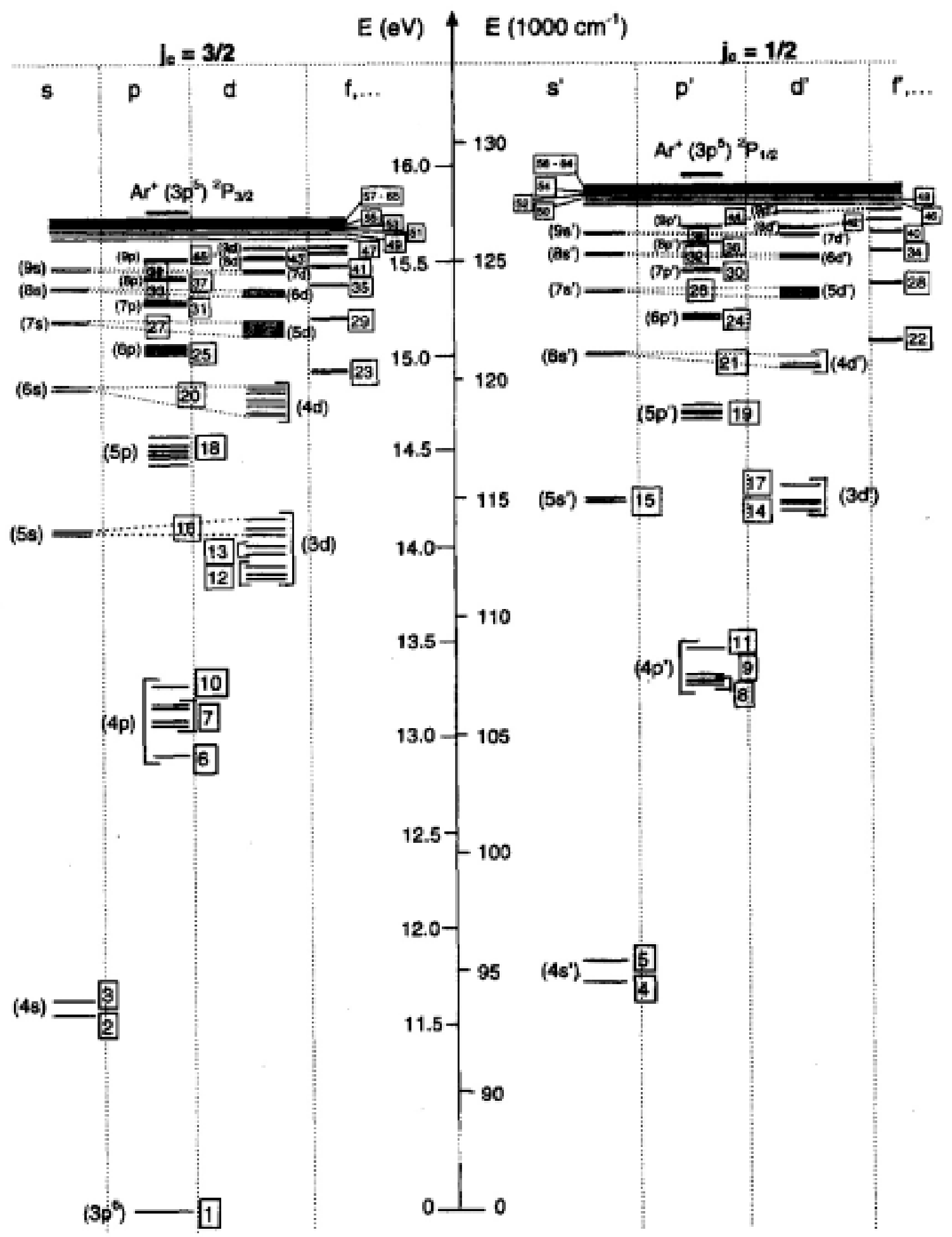

Figure 5.1 Energy level diagram of the argon atom numbered according to the levels used in the CR model. $^{8}$ 
The density of the ground state $(n=1)$ at the edge of the plasma is calculated using the ideal gas law, $n_{1}=P / k T$, where $P$ and $T$ are the source pressure and neutral argon temperature, respectively. A non-uniform ground state density spatial profile can be defined in the model. The sixty-four equations describing the densities of the excited states based on all included processes are simultaneously solved at each time step until a convergence is reached. The processes included in our version of the CR model are:

1. Electron impact excitation and de-excitation between all levels.

2. Thermal argon atom impact excitation and de-excitation between all levels.

3. Radiative decay and photo-excitation between all levels.

4. Electron impact ionization from and three-body recombination to all levels.

5. Thermal argon atom impact ionization from and three-body recombination to all levels.

6. Radiative recombination to all levels.

7. Diffusion and consequent de-excitation at the walls of metastable levels. (The lifetime of the other levels makes diffusion negligible.)

8. Two- and three-body collisions with thermal ground state atoms for metastable levels. (The densities of the other levels make these types of collisions negligible.)

9. Metastable-metastable collisions causing ionization of one of the atoms. (The densities of the other levels make these types of collisions negligible.)

All processes, except diffusion, are calculated locally at each radial position. The calculation of parameters for each of the above processes is given below, as described by Bogaerts et al. ${ }^{8}$ 
The cross section for electron impact excitation from level $n$ to level $m$, for an electron with energy $E$ is given by $\sigma_{\text {excit,e }}(n, m, E)=\sigma^{A}(n, m, E)+\sigma^{F}(n, m, E)$, where the cross section for optically allowed $(\Delta l= \pm 1, \Delta \mathrm{J}=0, \pm 1$, except for $\mathrm{J}=0 \rightarrow \mathrm{J}=0)$ transitions is approximated by

$$
\sigma^{A}(n, m, E)=4 \pi a_{0}^{2}\left(\frac{\varepsilon_{1}^{H}}{E_{m n}}\right)^{2} f_{m n} \alpha_{m n}^{A}\left(\frac{E}{E_{m n}}\right)^{-2}\left(\frac{E}{E_{m n}}-1\right) \ln \left(1.25 \beta_{m n} \frac{E}{E_{m n}}\right)
$$

and the cross section for parity-forbidden, $P$, and spin-forbidden, $S$, transitions, respectively, is

$$
\sigma^{F}(n, m, E)=\sigma^{P}(n, m, E)=4 \pi a_{0}^{2} \alpha_{m n}^{P}\left(\frac{E}{E_{m n}}\right)^{-1}\left(1-\left(\frac{E}{E_{m n}}\right)^{-1}\right)
$$

and

$$
\sigma^{F}(n, m, E)=\sigma^{S}(n, m, E)=4 \pi a_{0}^{2} \alpha_{m n}^{S}\left(\frac{E}{E_{m n}}\right)^{-3}\left(1-\left(\frac{E}{E_{m n}}\right)^{-2}\right)
$$

where $a_{0}$ is the Bohr radius, $\varepsilon_{l}{ }^{H}$ is the ionization energy of ground state hydrogen, $E_{m n}$ is the energy difference between levels $m$ and $n\left(E_{m n}=E_{m}-E_{n}\right), f_{m n}$ is the oscillator strength of the transition, and $\alpha_{m n}$ and $\beta_{m n}$ are transition-dependent parameters. For the optically forbidden transitions between the first four excited levels, the cross sections are given by

$$
\sigma_{\text {excit }, e}(n, m, E)=\frac{g_{m}}{g_{n}} \frac{E-E_{m n}}{E} 5.797 \times 10^{-15} Q_{m n}\left(E-E_{m n}\right)^{-0.54},
$$

where $Q_{23}=1, Q_{24}=Q_{25}=Q_{34}=Q_{35}=0.1$, and for $n=4, m=5$,

$$
\sigma_{e x c i t, e}(n, m, E)=\frac{g_{m}}{g_{n}} \frac{E-E_{m n}}{E} 8.111 \times 10^{-16}\left(E-E_{m n}\right)^{-1.04}
$$


To find the cross section for electron impact de-excitation, the principle of detailed balancing for $e^{-}(E)+\operatorname{Ar}(n) \underset{\text { excexcit }}{\stackrel{\text { exit }}{\rightleftarrows}} e^{-}\left(E^{\prime}\right)+\operatorname{Ar}(m)$ is used, where $E^{\prime}=E-E_{m n}$. This yields

$$
\sigma_{\text {de-excite },}\left(m, n, E^{\prime}\right)=\frac{g_{n} E}{g_{m} E^{\prime}} \sigma_{\text {excit }, e}(m, n, E)
$$

where $g_{n}$ and $g_{m}$ are the level degeneracies of levels $n$ and $m$, respectively.

The cross section for thermal atom impact excitation is given by

$$
\sigma_{\text {excit } t h}(n, m, E)=b_{n m}\left[E_{\text {thermal }}-E_{n m}\right],
$$

where $b_{23}=b_{45}=1.79 \times 10^{-20}\left(E_{n m}\right)^{-2.26}, b_{24}=b_{25}=b_{34}=b_{35}=4.8 \times 10^{-22}\left(E_{n m}\right)^{-2.26}$, and $b_{n m}=8.69 \times 10^{-}$ ${ }^{18}\left(E_{n m}\right)^{-2.26}$ for all other $n, m$, and $E_{\text {thermal }}$ is the energy of the thermal argon atoms. We have used $E_{\text {thermal }}=0.03 \mathrm{eV}$ based on LIF measurements of argon neutral temperatures. This process will only occur when the energy of the thermal atom is greater than that of the transition $\left(E_{\text {thermal }}>E_{n m}\right)$. For thermal atom impact de-excitation, the principle of detailed balancing is used such that

$$
\sigma_{d e-e x c i t, t h}\left(m, n, E^{\prime}\right)=\frac{g_{n} E}{g_{m} E^{\prime}} \sigma_{\text {excit th }}(m, n, E) .
$$

Only optically allowed transitions are considered for de-excitation.

The rate of radiative decay is calculated by multiplying the Einstein transition probability, $A$, by an escape factor, $\Lambda$, which takes into account radiation trapping in which emitted radiation can be absorbed, re-exciting the atom via photoexcitation. The effective transition probability for each level is calculated by

$$
\bar{A}(m, n)=\sum_{x} \sum_{y} g(y) A(y, x) / \sum_{y} g(y),
$$

where $x$ and $y$ denote the levels belonging to the upper effective level, $n$, and lower effective level, $m$, respectively. Photoexcitation is only a factor in the ground state, $n=1$, 
due to the high density, thus the escape factor is assumed to be one for all excited levels, $n>1$. For transitions to the ground state, the escape factors are given by

$$
\Lambda(m, 1)=1.9 T_{D} \exp \left(\frac{-\pi T_{C D}^{2}}{4 T_{C}^{2}}\right)+1.3 T_{C} \operatorname{erf}\left(\frac{\sqrt{\pi} T_{C D}}{2 T_{C}}\right)
$$

where $T_{D}=\frac{1}{k_{0} R \sqrt{\pi \ln \left(k_{0} R\right)}}$ is the transmission coefficient for pure Doppler broadening, $T_{C}=\sqrt{\frac{a}{\sqrt{\pi} k_{0} R}}$ is the transmission coefficient for pure collisional broadening, and $T_{C D}=\frac{2 a}{\pi \sqrt{\ln \left(k_{0} R\right)}}$ is the coefficient for collisionally broadened emission and Doppler broadened absorption. These coefficients describe a cylindrical tube of radius $R$, with optical depth pertaining to the line center,

$$
k_{0} R=\frac{2.1 \times 10^{-17} g(m)}{\left[E_{\text {excit }}(m)\right]^{3} \sqrt{T_{\text {gas }}}} A(m, 1) N_{\text {gas }} R
$$

and damping coefficient,

$$
a=A(m, 1)\left[1+\frac{3.225 \times 10^{-14}}{\left[E_{\text {excit }}(m)\right]^{3}} g(m) N_{\text {gas }}\right] \frac{4.839 \times 10^{-9}}{E_{\text {excit }}(m) \sqrt{T_{\text {gas }}}}
$$

where $E_{\text {excit }}(m)$ is the excitation energy of level $m, T_{\text {gas }}$ and $N_{g a s}$ are the gas temperature (in Kelvin) and atomic ground state density, respectively.

The cross section for electron impact ionization from the ground state $(n=1)$ is given by

$$
\sigma_{\text {ioniz }, e}(n=1, E)=10^{-16} \frac{23.9 \mathrm{~cm}^{2} \mathrm{eV}}{E} \ln \left(\frac{E+\frac{150 \mathrm{eV}^{2}}{E}}{E_{\text {ioniz }}(n=1)}\right) 4.6\left(\arctan \left(\frac{a-\varepsilon}{4.6 \mathrm{eV}}\right)-\arctan \left(\frac{-\varepsilon}{4.6 \mathrm{eV}}\right)\right)
$$


where $a=\left[E-E_{\text {ioniz }}(n=1)\right] / 2$ and $\varepsilon=1.2 \mathrm{eV}-250 \mathrm{eV}^{2} /\left[E+2 E_{\text {ioniz }}(n=1)\right]$. For all excited levels $(n>1)$, the electron impact ionization cross sections are given by

$$
\sigma_{\text {ioniz }, e}(n, E)=4 \pi a_{0}^{2}\left(\frac{\varepsilon_{1}^{H}}{E_{\text {ioniz }}(n)}\right)^{2} \alpha_{n}\left(\frac{E}{E_{\text {ioniz }}(n)}\right)^{-2}\left(\frac{E}{E_{\text {ioniz }}(n)}-1\right) \ln \left(1.25 \beta_{n} \frac{E}{E_{\text {ioniz }}(n)}\right)
$$

where $E_{\text {ioniz }}(n)$ is the ionization energy of the effective level $n$.

Three-body recombination occurs when an electron impacts an argon ion and an additional electron joins with the ion to create a neutral argon atom. Because this is the opposite process of electron impact ionization, the principle of detailed balancing for $e^{-}(E)+A r(n) \underset{3 b-\text { recomb }}{\stackrel{\text { ioniz }}{\rightleftarrows}} e^{-}\left(E^{\prime}\right)+A r^{+}+e_{b}^{-}\left(E_{b}\right)$ is used to calculate the cross section given by

$$
\sigma_{3 b-r e c o m b}\left(n, E^{\prime}\right)=n_{e} \frac{g_{n}}{2 g_{1}^{+}}\left(\frac{h^{2}}{2 \pi m_{e} k T_{e}}\right)^{3 / 2} \frac{E}{E^{\prime}} \sigma_{\text {ioniz }}(n, E),
$$

where $E^{\prime}=E-E_{\text {ioniz }}(n), n_{e}, m_{e}$, and $T_{e}$ are the electron density, mass, and mean energy, respectively, $g_{1}{ }^{+}$is the statistical weight of the ion ground state with $g_{1}{ }^{+}=1$ for the ground state, $g_{1}{ }^{+}=4$ for the unprimed (parent ion has total angular momentum of $3 / 2$ ) system, and $g_{1}{ }^{+}=2$ for the primed (parent ion has total angular momentum of $1 / 2$ ) system, $h$ is Planck's constant, and $k$ is Boltzmann's constant, and the energy of the bound electron, $E_{b}$, is assumed to be zero. While the conventional formula used for three-body recombination has a different dependence on electron temperature, ${ }^{9}$ the electron temperature dependence in Eq. (5.15) arises from balancing, in steady state, the Saha equation for the ionization rate with the three-body recombination rate in the principle of detailed balancing. ${ }^{10}$ 
Radiative recombination of an electron and argon ion is the inverse process of photoionization, described by $A r(n)+h v \underset{\text { rad.recomb }}{\stackrel{\text { photoiniz }}{\rightleftarrows}} A r^{+}+e^{-}(E)$. Thus, the cross section for radiative recombination is given by

$$
\sigma_{\text {rad.recomb }}(n, E)=\frac{g_{n}}{2 g_{1}^{+}} \frac{(h v)^{2}}{m_{e} c^{2}} \frac{1}{E} \sigma_{\text {photoioniz }}(n, h v),
$$

where $E=h v-E_{\text {ioniz }}(n), c$ is the speed of light, $h v$ is the energy of the photon, and

$$
\sigma_{\text {photoioniz }}(n, h v)=\left\{\begin{array}{l}
3.5 \times 10^{-17} \mathrm{~cm}^{2} \quad n=1, \varepsilon_{1} \leq h v \leq 2 \varepsilon_{1}^{H} \\
2.8 \times 10^{-16} \mathrm{~cm}^{2}\left(\frac{\varepsilon_{1}^{H}}{h v}\right)^{3} \quad h v>2 \varepsilon_{1}^{H} \\
2 \times 10^{-18} \mathrm{~cm}^{2} \gamma_{m n} \quad 2 \leq m \leq 5, \bar{\varepsilon}_{4 s} \leq h v \leq 0.59 \varepsilon_{1}^{H}, \\
7.91 \times 10^{-18} \mathrm{~cm}^{2} \gamma_{m n} \quad 2 \leq m \leq 5, h v>0.59 \varepsilon_{1}^{H} \\
\sum_{n} 7.91 \times 10^{-18} \mathrm{~cm}^{2} \gamma_{m n}\left(\frac{\varepsilon_{m}}{\varepsilon_{1}^{H}}\right)^{2.5}\left(\frac{\varepsilon_{1}^{H}}{h v}\right)^{3} \quad m \geq 6
\end{array}\right.
$$

where $\gamma_{m n}$ is a transition-dependent parameter and $\bar{\varepsilon}_{4 s}$ is the mean ionization energy of the $4 \mathrm{~s}$ and $4 \mathrm{~s}^{\prime}$ states. ${ }^{7}$ Similar to photoexcitation, photoionization only plays a factor for the ground state.

The loss of metastable atoms ( $n=2$ and $n=4)$ due to diffusion is described by the diffusion coefficient,

$$
D=\frac{k T_{g a s}}{m_{A r} v}
$$

where $v$ is the atom-atom collision frequency. Tachibana experimentally found $D$ for the argon metastables to be $2.4 \times 10^{18} \mathrm{~cm}^{-1} \mathrm{~s}^{-1} / n$, where $n$ is the ground state density. ${ }^{11}$ Using the ideal gas law, $n=N / V=P / k T$ such that $D$ at 1 Torr and $300 \mathrm{~K}$ is $74.6 \mathrm{~cm}^{2} \mathrm{~s}^{-1}$. The CR model uses a linear equation based on this value to calculate $D$ using the input 
pressure and gas temperature. For all cases we assume the gas temperature is $348 \mathrm{~K}$ (room temperature) based on Ar I LIF measurements.

For two- and three-body collisions with thermal ground state atoms, the rate coefficients are $k_{2 b}=2.3 \times 10^{-15} \mathrm{~cm}^{3} \mathrm{~s}^{-1}$ and $k_{3 b}=1.4 \times 10^{-32} \mathrm{~cm}^{6} \mathrm{~s}^{-1}$ for $n=2$ and $k_{2 b}=4.3 \times 10^{-15} \mathrm{~cm}^{3} \mathrm{~s}^{-1}$ and $k_{3 b}=1.5 \times 10^{-32} \mathrm{~cm}^{6} \mathrm{~s}^{-1}$ for $n=4$. For metastable-metastable atom collisions, the rate coefficient is $k_{m e t}=6.4 \times 10^{-10} \mathrm{~cm}^{3} \mathrm{~s}^{-1}$, used for both $n=2$ and $n=4$. These processes are only important for the metastable states because they require high state densities that are only present for metastables.

The rate of gain of atoms to effective level $n$ at radial position $r$ is

$$
\begin{aligned}
& R_{\text {gain }}(n, r)=\sum_{l=1}^{n-1} \sum_{E}\left[\left(\sigma_{\text {excit }, e}(l, n, E) f_{e}(r, E)+\sigma_{\text {excit } t h}(l, n, E) v_{\text {th }} N(1, r)\right) N(l, r)\right] \\
& \left.+\sum_{m=n+1}^{65} \sum_{E}\left[\begin{array}{l}
\sigma_{d e-\text { excit, },}(m, n, E) f_{e}(r, E)+\sigma_{d e-e x c i t, t h}(m, n, E) v_{t h} N(1, r)+ \\
A(m, n) \Lambda(m, n)
\end{array}\right) N(m, r)\right]
\end{aligned}
$$

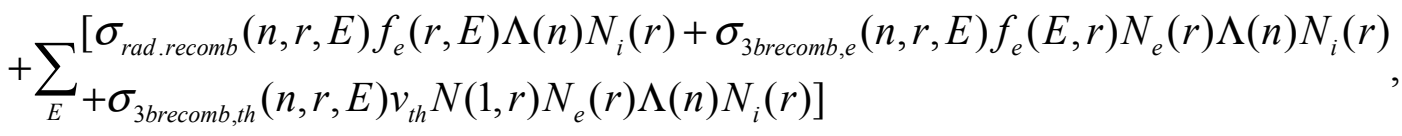

where $f_{e}$ is the radially dependent electron energy distribution function $(\mathrm{EEDF}), v_{\text {th }}=\sqrt{2 E_{\text {thermal }} / m_{A r}}$ is the velocity of thermal argon atoms, $N(m, r)$ is the density of effective level $m$ at position $r$, and $N_{i}$ is the ion density, assumed to be equal to the electron density by quasi-neutrality. 
The rate of loss of atoms from effective level $n$ at radial position $r$ is given by

$$
\begin{aligned}
R_{\text {loss }}(n, r) & =\sum_{m=n+1}^{65} \sum_{E}\left[\left(\sigma_{\text {excit }, e}(n, m, E) f_{e}(r, E)+\sigma_{\text {excit th }}(n, m, E) v_{t h} N(1, r)\right) N(n, r)\right] \\
& +\sum_{l=1}^{n-1} \sum_{E}\left[\left(\begin{array}{l}
\sigma_{\text {de-excit }, e}(n, l, E) f_{e}(r, E)+\sigma_{\text {de-excit }, t h}(n, l, E) v_{t h} N(1, r)+ \\
A(n, l) \Lambda(n, l)
\end{array}\right) N(n, r)\right] \\
& +\sum_{E}\left[\left(\sigma_{\text {ion,e }}(n, r, E) f_{e}(r, E)+\sigma_{\text {ion, }, t h}(n, r, E) v_{t h} N(1, r)\right) N(n, r)\right] \\
& +\left(k_{2 b} N(1, r)+k_{3 b} N^{2}(1, r)+k_{\text {met }}(N(2, r)+N(4, r))\right) N(n, r) .
\end{aligned}
$$

The equation used to describe the change of density of state $n$ at position $r, N(n, r)$, versus time is then

$$
\frac{d N(n, r)}{d t}=R_{\text {gain }}(n, r)-R_{\text {loss }}(n, r)+D \frac{d^{2} N(n, r)}{d r^{2}} .
$$

The electron energy distribution functions (EEDFs) used in the code were based on RF-compensated Langmuir probe measured electron density and temperature radial profiles. As a first step, Maxwellian EEDFs were created based on the measured electron temperatures. A spatially localized, energetic electron beam could then be added to the Maxwellian distribution to study the effects of a beam on the neutral argon excited levels. The radial profiles of electron density, the EEDFs, and the fill pressure measured at the edge of the chamber are input variables for the code. The radial profile of the neutral ground state density in the code is varied to find the ground state profile that is most consistent with the spectroscopic and fluorescence measurements. The percent of depletion at the center, $P$, and depletion width, $W$, are used to parameterize the neutral profile according to 


$$
\begin{aligned}
N(r)= & N_{0}\left[m\left(\frac{2 r}{W}\right)^{2}+(1-P)\right],-\frac{W}{2} \leq r \leq \frac{W}{2}, \\
& m=P \text { for } P \leq 0.5, \\
& m=1-P \text { for } P>0.5, \\
N(r)= & N_{0},|r|>\frac{W}{2}
\end{aligned}
$$

where $N_{0}$ is the neutral density found using the ideal gas law based on the input pressure and room temperature at the walls of the chamber. An example profile with $P=0.5$ and $W=3$ is shown in Figure 5.2. Because the Langmuir probe measurements were only obtained for half the diameter of the plasma, cylindrical symmetry is assumed in the CR model.

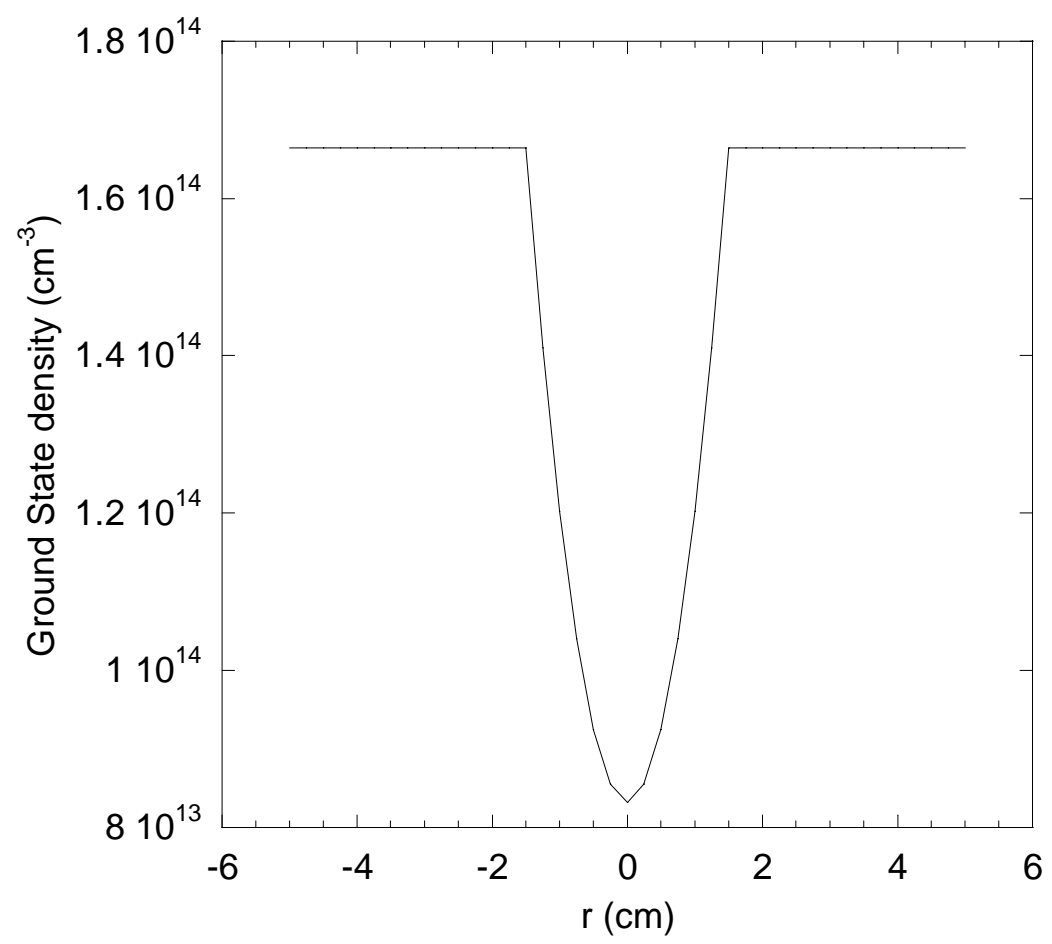

Figure 5.2 Example ground state profile using Eq. (5.21) with $P=0.5$ and $W=3.0$. 
For capacitive discharges, another ground state profile function was also used to test for off-axis depletion of the neutrals. The profile, similar to Equation 5.21, is given by

$$
\begin{aligned}
N(r)= & N_{0}\left(m\left(\frac{2(r+C)}{W}\right)^{2}+(1-P)\right), \quad-C-W / 2 \leq r \leq-C+W / 2 \\
N(r)= & N_{0}\left(m\left(\frac{2(r-C)}{W}\right)^{2}+(1-P)\right), C-W / 2 \leq r \leq C+W / 2, \\
& m=P \text { for } P \leq 0.5, \\
& m=1-P \text { for } P>0.5, \\
N(r)= & N_{0}, \quad|r|>W / 2,-C+W / 2<r<C-W / 2
\end{aligned}
$$

where $C$ is the center of the off-axis depletion, another variable to describe the ground state density profile. An example profile with $P=0.4, W=2.0$, and $C=1.0$ is shown in Figure 5.3.

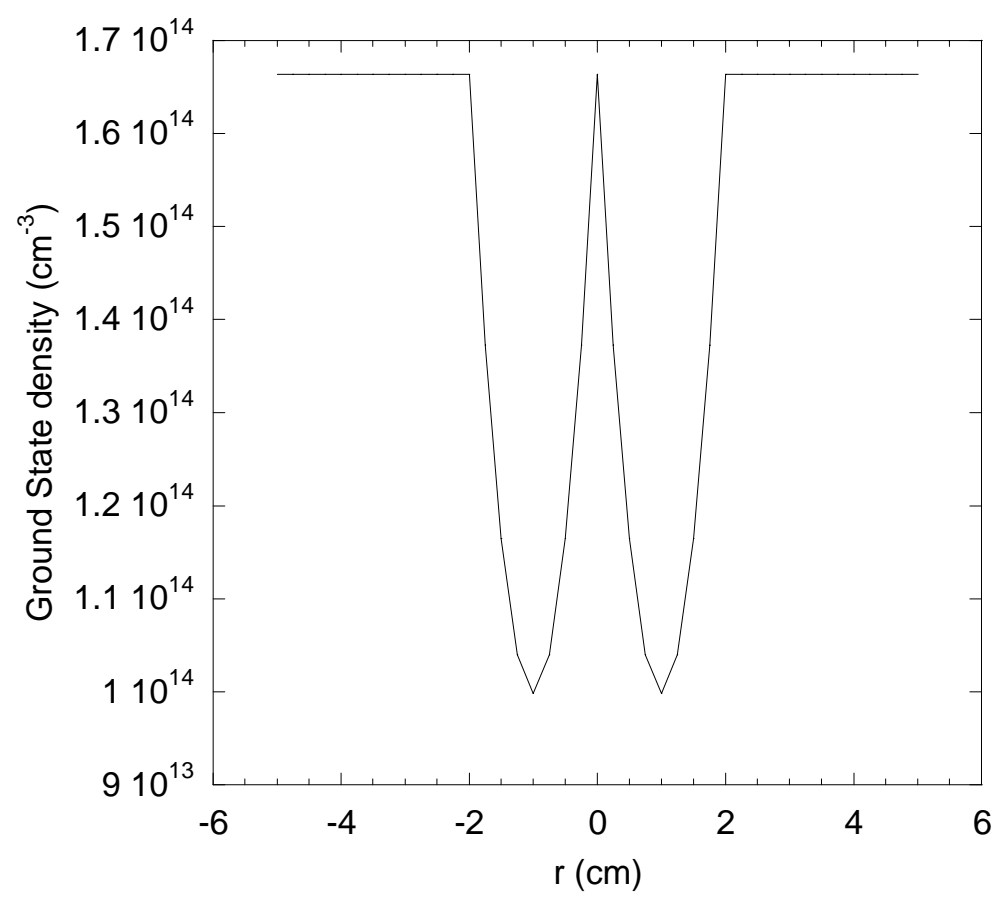

Figure 5.3 Example ground state profile using Eq. (5.22) with $P=0.4, W=2.0$, and $C=1.0$. 


\section{References}

${ }^{1}$ R. Boswell, R. Porteous, A. Prytz, A. Bouchoule, and P. Ranson, Phys. Lett. A 91, 163 (1982).

2 J. Wang, W. Wu, W. Sun, H. Deng, X. Cheng, and Y. Tang, High Power Laser and Particle Beams 17, $1513(2005)$.

${ }^{3}$ R.F. Boivin, "Spectroscopy System and Basic Spectroscopy Diagnostics for the HELIX and LEIA Plasma Devices," PL-046.

${ }^{4}$ C.E. Moore, Atomic Energy Levels, Vol 1, Natl. Bur. Stds., NSRDS-NBS 35 (1971).

${ }^{5}$ Rundel R. D. and R. F. Stebbings, The Role of Metastables Particles in Collision Processes in Case Studies in Atomic Collision Physics II, (North Holland, 1972).

${ }^{6}$ B. van der Sijde, J.J.A.M. van der Mullen, and D.C. Schram, Beitr. Plasmaphys. 24, 447 (1984).

${ }^{7}$ J. Vlček, J. Phys. D: Appl. Phys. 22, 623 (1989).

${ }^{8}$ A. Bogaerts, R. Gijbels, and J. Vlcek, J. Appl. Phys. 84, 121 (1998).

${ }^{9}$ J.D. Huba, NRL Plasma Formulary, (Naval Research Laboratory, 1998).

${ }^{10}$ M. Mitchner and C.H. Kruger, Jr., Partially Ionized Gases, (Wiley, 1973). Also available at http://navier.stanford.edu/PIG/PIGdefault.html.

${ }^{11}$ K. Tachibana, Phys. Rev. A 34, 1007 (1986). 


\section{Chapter 6: Measurements of Radial Profiles, Temperatures and Flows in HELIX}

In this chapter, measurements of neutral temperature, the excited neutral state radial profile, electron temperature, and electron density in HELIX obtained with LIF, passive emission spectroscopy, and the Langmuir probe are presented for three different plasma source configurations. The excited state radial profiles obtained from the matrix form of the Abel inversion of the emission spectroscopy measurements are also included for each source configuration. Complete radial profiles were obtained for operation of the source in the helicon mode (6.1 Parameter Set A) and in the capacitive mode (6.2 Parameter Set B). 6.3 Parameter Set C is also for a helicon mode plasma, but the signal to noise was not sufficient for a complete LIF radial profile. For a limited set of plasma parameters, flow measurements of neutrals and ions were also obtained as part of an investigation of diffusion and momentum coupling to neutrals in helicon plasmas. Finally, time resolved measurements of neutral density and temperature for a single source configuration are also presented as part of an investigation of the ionization fraction as a function of time in a pulsed helicon plasma.

As noted previously, Langmuir probe measurements were obtained for only one side of the discharge. Both the line integrated emission spectroscopy (States 10 and 11) and LIF measurements (State 3) were obtained across the entire plasma column diameter. However, the inversions of the emission spectroscopy data assume intrinsic cylindrical symmetry. Remarkably, the LIF measurements indicate a significant amount of asymmetry across the plasma column; more than just a simple shift of the peak LIF emission away from $r=0$. Simple shifts in the peak of the plasma density, or excited 
neutral state density, are also visible in the emission spectroscopy data reported in this work and have been reported in other helicon source experiments. ${ }^{1}$ However, it is possible that the large asymmetries seen in these LIF measurements are an artifact of the lengthy time required to obtain the LIF data, i.e., the plasma conditions may have varied over the many hours needed to make the measurements. Therefore, consistent with the spatial coverage of the Langmuir probe measurements (required for the CR model), the LIF and emission spectroscopy measurements in this work will be compared to the CR model results only for the half of the discharge for which Langmuir probe data are available.

Another possible artifact is the increase in state density at the edge of the Abel inverted profiles that are presented later in this chapter. Because the viewport limits the value of $R_{\max }$ in the matrix inversion technique, non-negligible contributions to the emission from larger plasma radii have been ignored and the state density at the edge of the plasma is overestimated in the Abel inversion results.

\subsection{Parameter Set A (6 mTorr Helicon mode)}

For a helicon mode plasma with a magnetic field strength of 750 Gauss, RF power of 300 Watts, gas flow of $114 \mathrm{sccm}$, fill pressure of $6.0 \mathrm{mTorr}$, and operating pressure (at the front Balzers gauge) of 18.7 mTorr, the radial profiles of electron density and temperature are shown in Figure 6.1. The radial profile of neutral temperature and State 3 relative density measured using LIF are shown in Figure 6.2. The LIF data were smoothed by averaging each neighboring pair of data points. The line-integrated density versus vertical position measured using the passive spectroscopy transitions are shown in 
Figure 6.3. Figure 6.4 shows the results of the Abel inversion for the $y>0$ emission data of Figure 6.3.
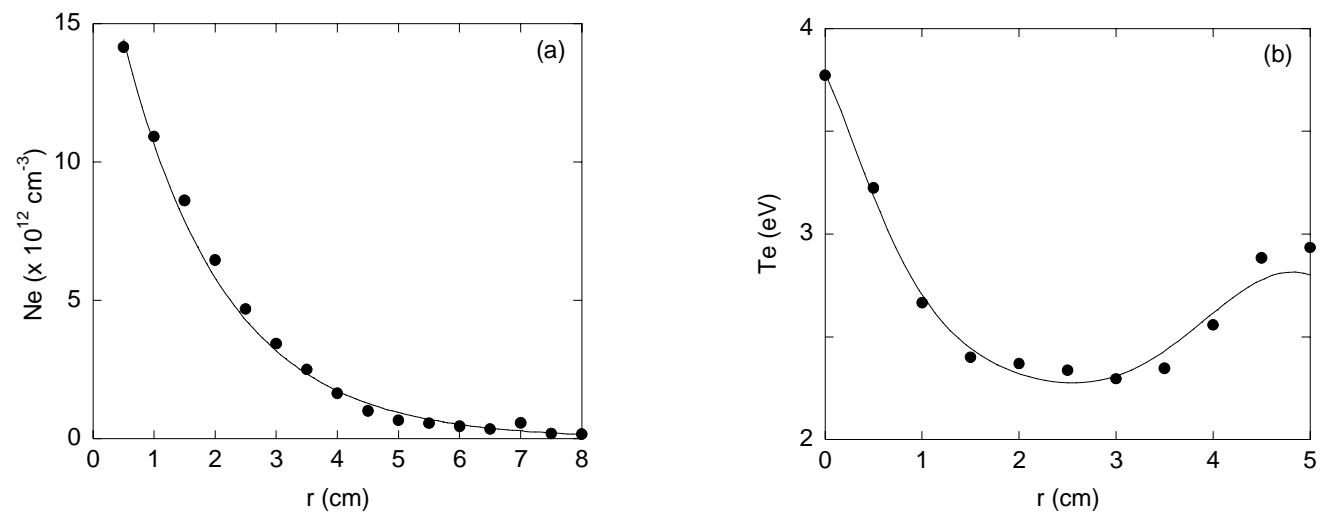

Figure 6.1 Langmuir probe data with the fits used for CR model inputs. a) Electron density. b) Electron temperature.
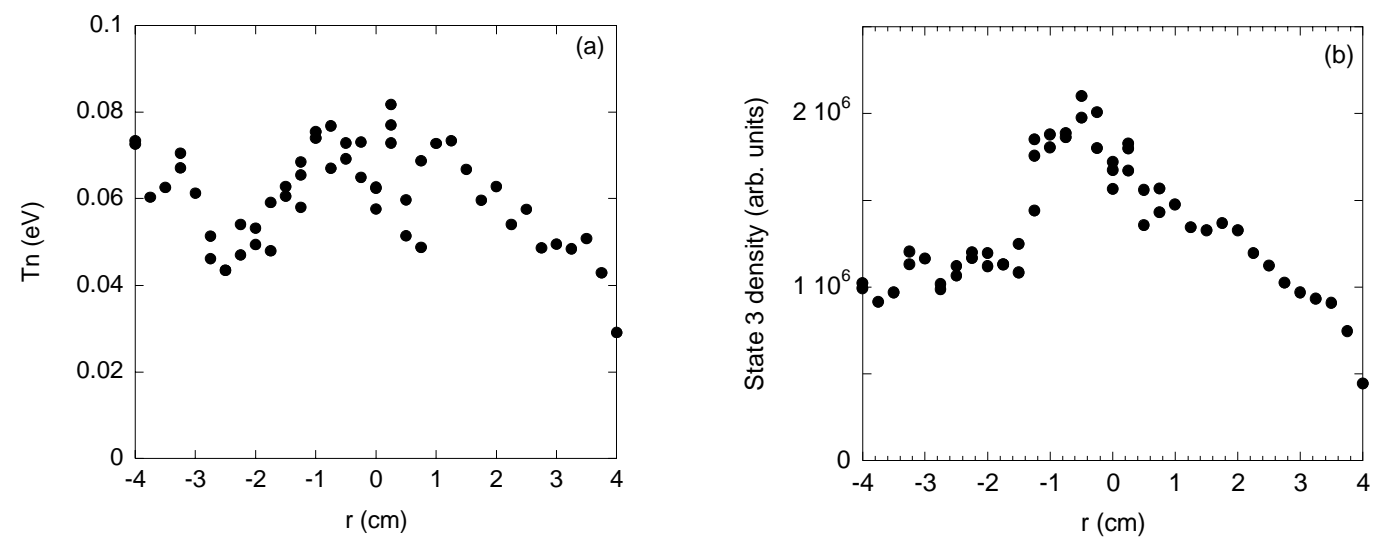

Figure 6.2 Radial profiles of a) neutral temperature and b) State 3 relative density measured with LIF. 

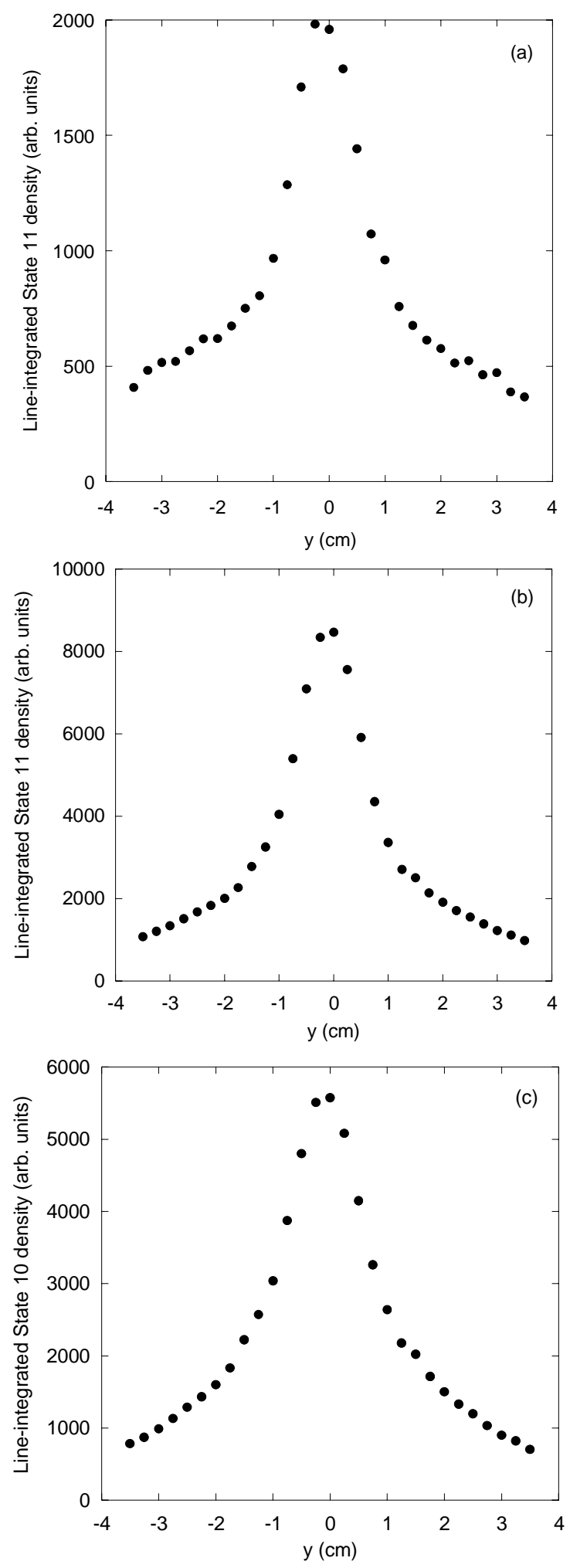

Figure 6.3 Line-integrated emission intensity versus vertical position using the a) $667.9 \mathrm{~nm}$, b) $750.6 \mathrm{~nm}$, and c) $751.7 \mathrm{~nm}$ transitions. 

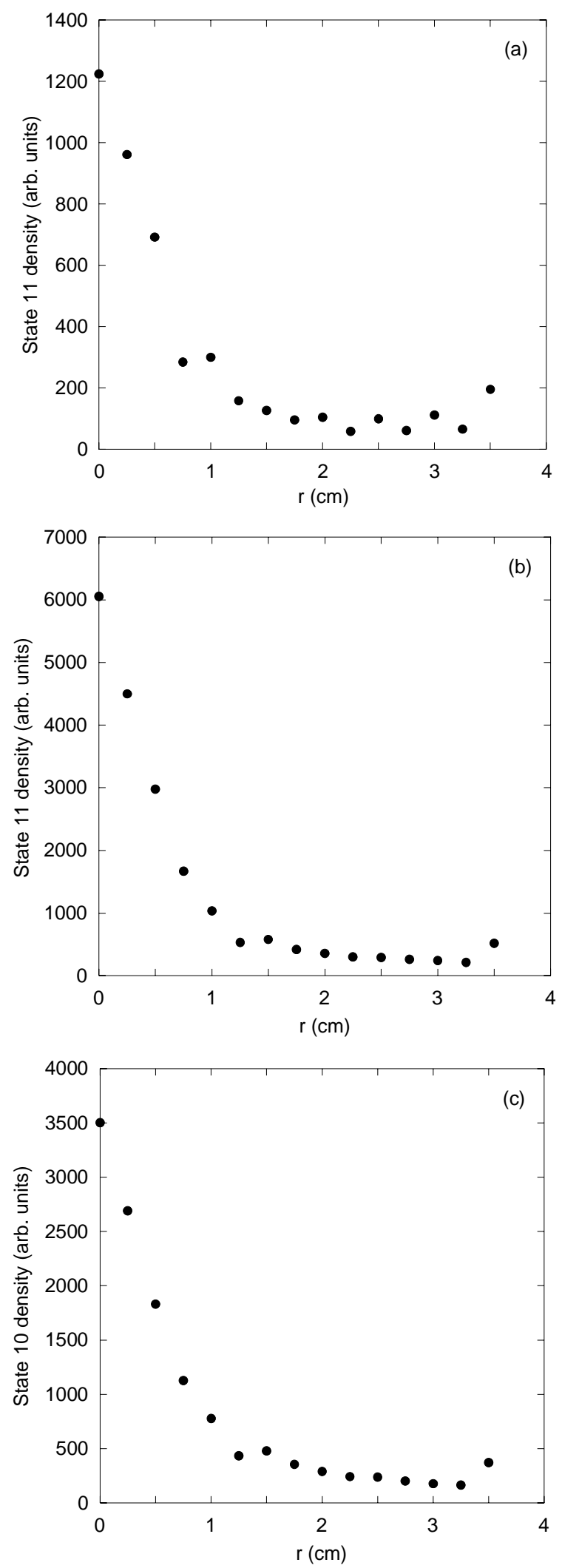

Figure 6.4 Radial profiles of relative densities obtained by Abel inversion of spectroscopy data for the a) $667.9 \mathrm{~nm}$ (State 11), b) $750.6 \mathrm{~nm}$ (State 11), and c) $751.7 \mathrm{~nm}$ (State 10) transitions. 


\subsection{Parameter Set B (Capacitive mode)}

For a capacitive mode plasma with a magnetic field strength of 750 Gauss, RF power of 200 Watts, gas flow of $114 \mathrm{sccm}$, fill pressure of $6.0 \mathrm{mTorr}$, and operating pressure of 13.1 mTorr, the radial profiles of electron density and temperature are shown in Figure 6.5. In contrast to the helicon mode, the electron temperature is peaked off-axis. The radial profile of State 3 relative density measured by LIF is shown in Figure 6.6. The LIF data were smoothed by averaging each neighboring pair of data points and the LIF intensity profile is surprisingly asymmetric. The line-integrated density versus vertical position measured using the passive spectroscopy transitions are shown in Figure 6.7. Figure 6.8 shows the results of the Abel inversion for the data of Figure 6.7. Because the emission spectroscopy data are also asymmetric, inversions were performed separately for the $y>0$ and the $y<0$ data. Despite the asymmetry in the line integrated emission data, all of the inversion yield relatively flat density profiles for States 10 and 11.
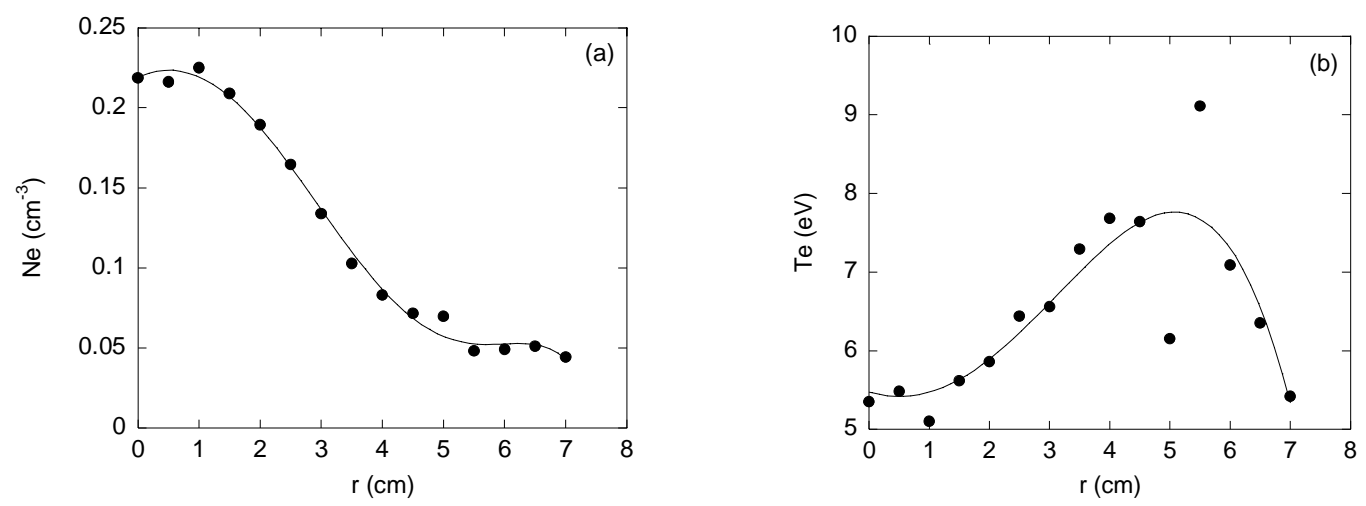

Figure 6.5 Langmuir probe data with the fits used for the CR model inputs. a) Electron density. b) Electron temperature. 

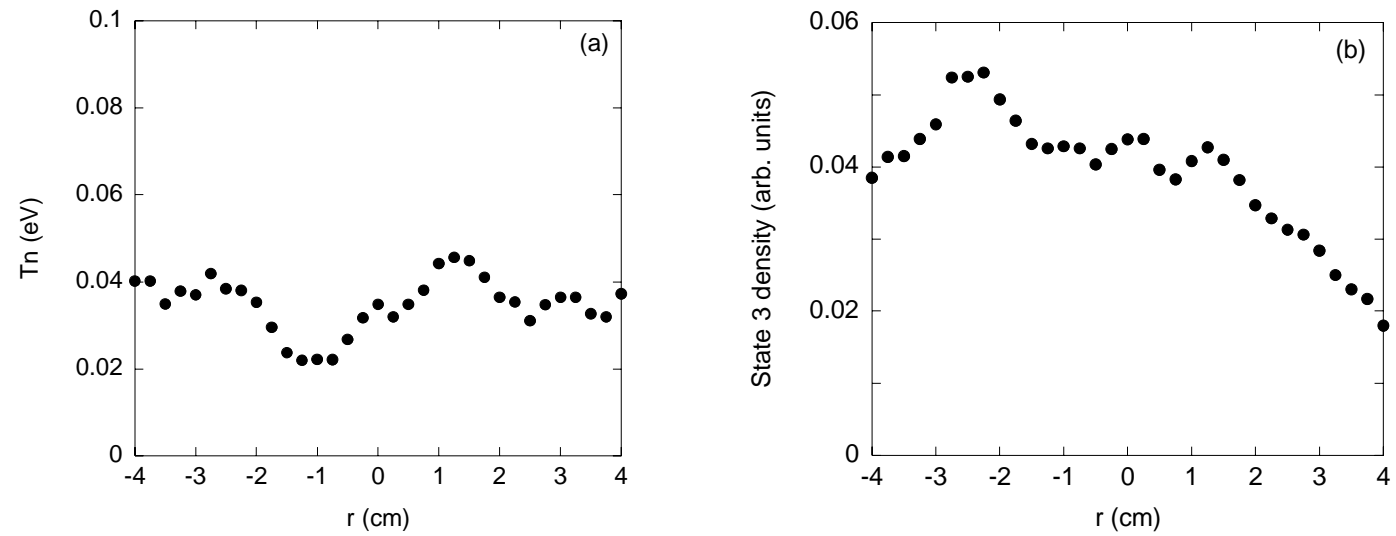

Figure 6.6 Radial profiles of a) neutral temperature and b) State 3 relative density measured with LIF. 

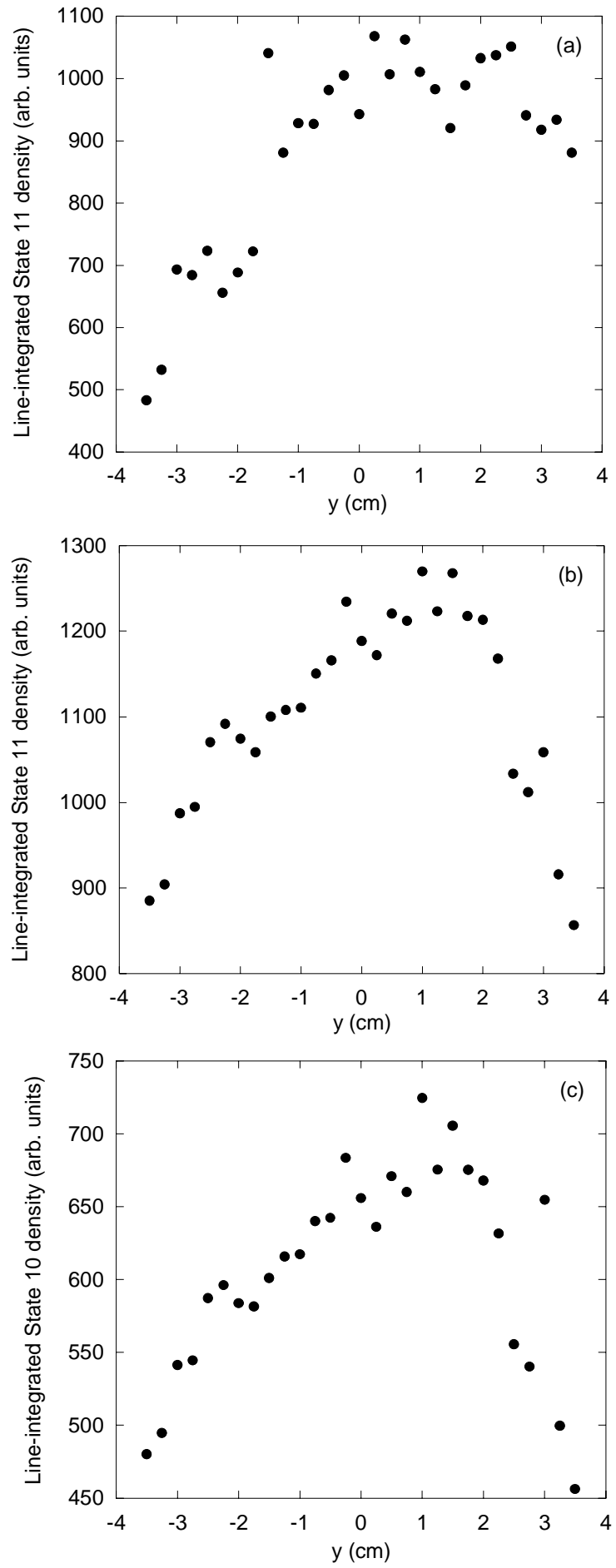

Figure 6.7 Line-integrated emission intensity versus vertical position using the a) $667.9 \mathrm{~nm}$, b) $750.6 \mathrm{~nm}$, and c) $751.7 \mathrm{~nm}$ transitions. 

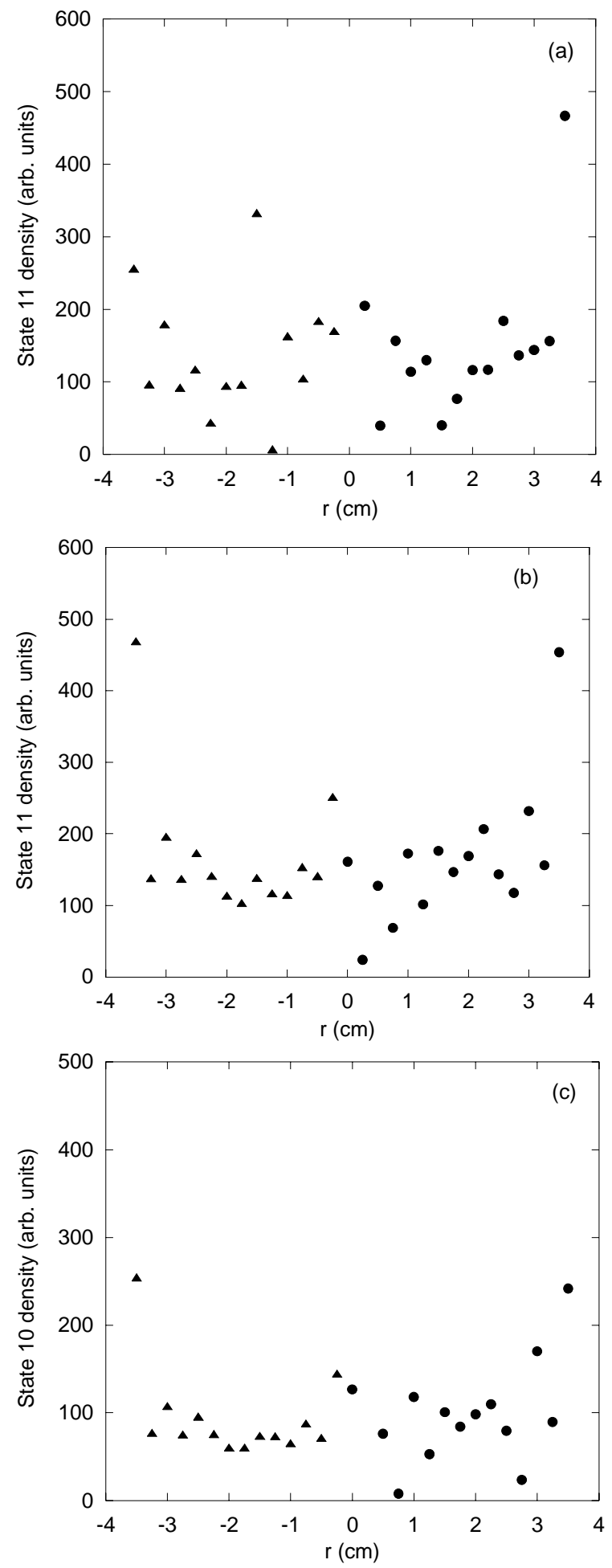

Figure 6.8 Radial profiles of relative densities obtained by Abel inversion of spectroscopy data for the a) $667.9 \mathrm{~nm}$ (State 11), b) $750.6 \mathrm{~nm}$ (State 11), and c) $751.7 \mathrm{~nm}$ (State 10) transitions. The profiles obtained using the $y>0$ (circles) and $y<0$ (triangles) data separately are shown. 


\subsection{Parameter Set C (5 mTorr Helicon mode)}

For a helicon mode plasma with a magnetic field of 750 Gauss, RF power of 350 Watts, gas flow of $102 \mathrm{sccm}$, fill pressure of $5.1 \mathrm{mTorr}$, and operating pressure of 15.1 mTorr, the electron density and temperature profiles are shown in Figure 6.9. LIF signal (shown in Figure 6.10) was only attainable for $1.5 \mathrm{~cm}<r<4.0 \mathrm{~cm}$ for these source parameters. The LIF data were smoothed by averaging each neighboring pair of data points. The line-integrated emission intensity versus vertical position measurements are shown in Figure 6.11. Because the emission data are highly symmetric, the separate Abel inversions of the $y>0$ and $y<0$ data are very similar. Only the emission profile from the $667.9 \mathrm{~nm}$ line has any noticeable asymmetry at the edge of the plasma. Figure 6.12 shows the results of the Abel inversion for the $y>0$ data of Figure 6.11 (the inversion results using the $y<0$ data for the $667.9 \mathrm{~nm}$ line are also included in Figure 6.11a for completeness).
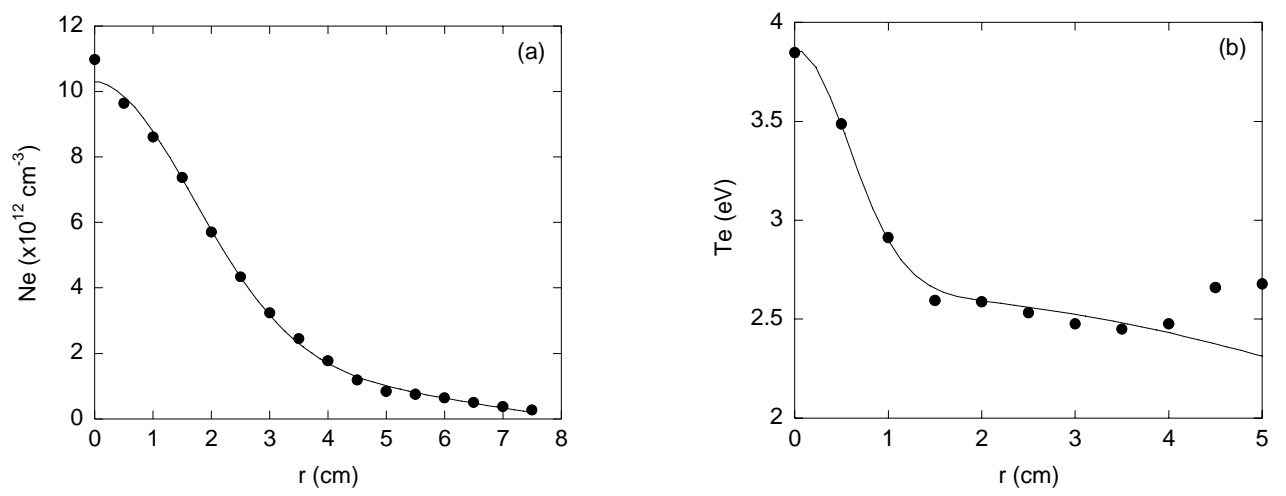

Figure 6.9 Langmuir probe data with the fits used for the CR model inputs. a) Electron density. b) Electron temperature. 


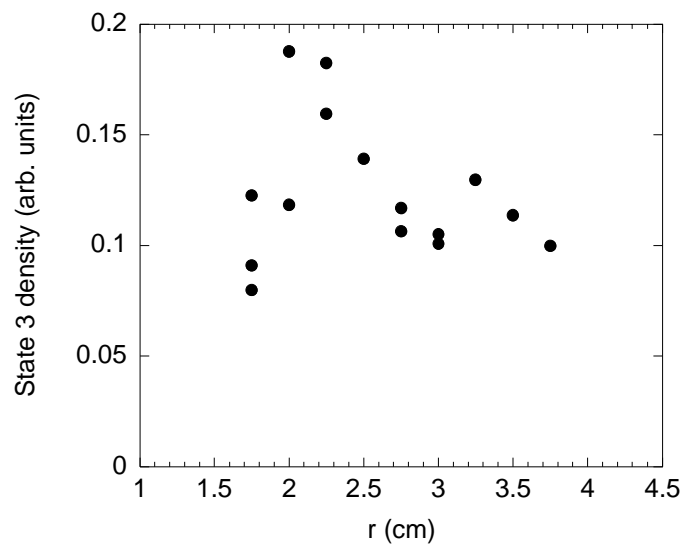

Figure 6.10 Radial profile of State 3 relative density measured with LIF. 

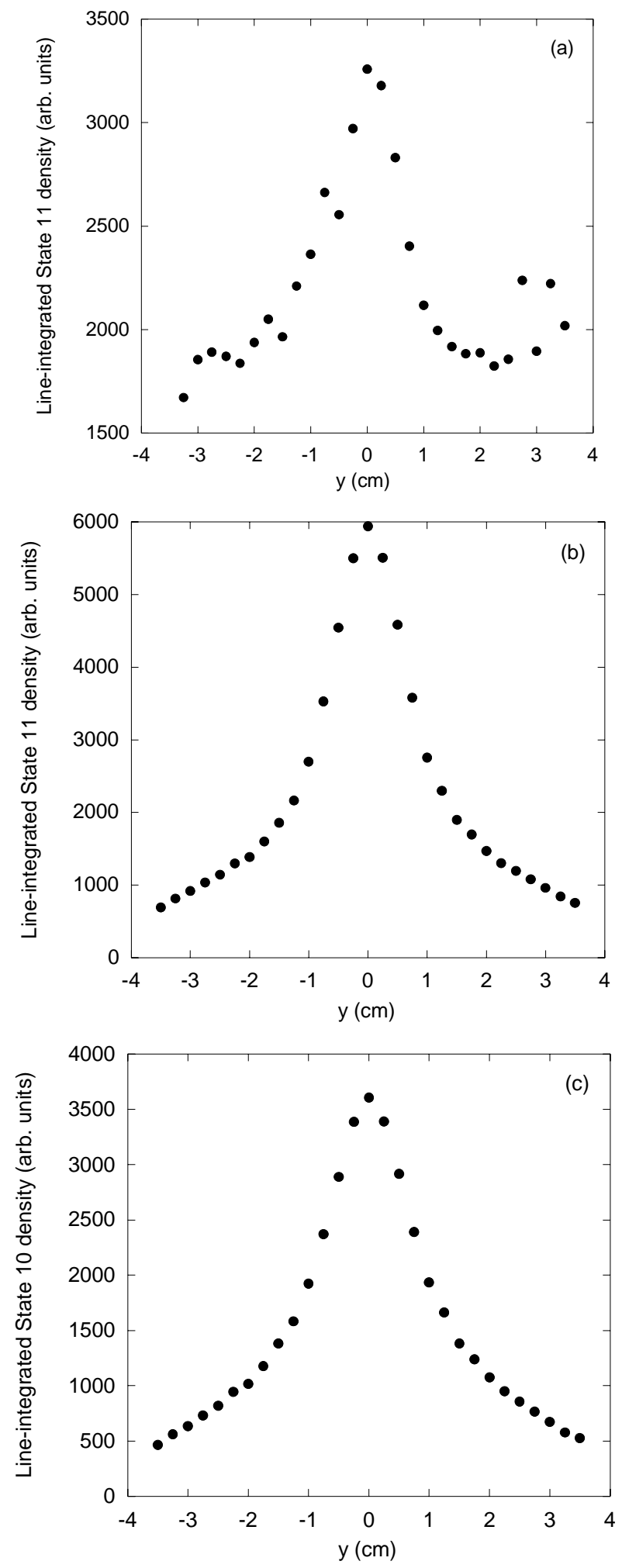

Figure 6.11 Line-integrated emission intensity versus vertical position using the a) $667.9 \mathrm{~nm}$, b) $750.6 \mathrm{~nm}$, and c) $751.7 \mathrm{~nm}$ transitions. 

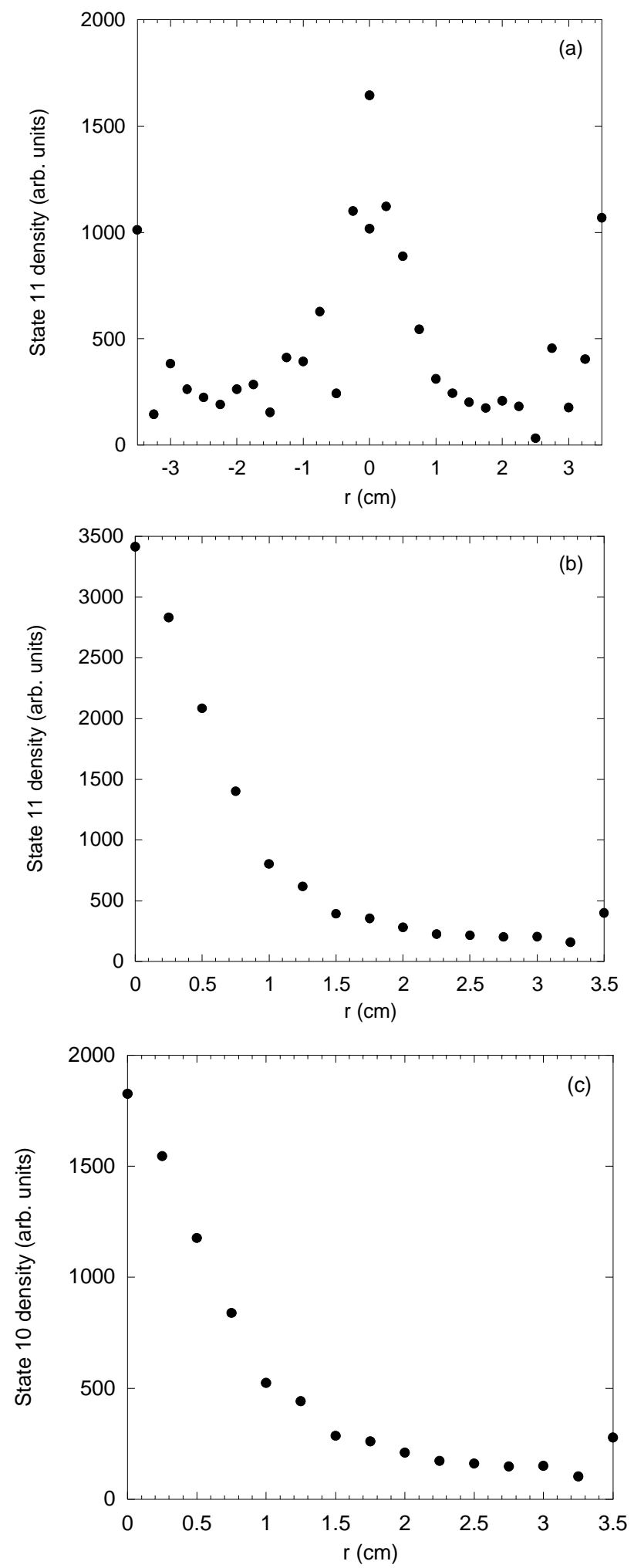

Figure 6.12 Radial profiles of relative state densities obtained by Abel inversion of spectroscopy data for the a) $667.9 \mathrm{~nm}$ (State 11), b) $750.6 \mathrm{~nm}$ (State 11), and c) $751.7 \mathrm{~nm}$ (State 10) transitions. The profiles obtained using both the $y>0$ and $y<0$ data are shown in a) and only those obtained using the $y>0$ are shown in b) and c). 


\subsection{Neutral and ion flow measurements}

Coupling of ion momentum, i.e. flow, to neutrals in plasma propulsion systems, such as the double layer driven plasma thruster, would permit the development of thrust without leading to the charging up of a spacecraft due to non-neutral energetic particle emission. Therefore, development of diode laser based LIF neutral flow measurement techniques for argon helicon plasmas would be of immediate use to the plasma thruster community. To explore the possibility of measuring neutral flows in a helicon source with the portable LIF diagnostic, radially resolved, perpendicular Ar I LIF measurements were obtained with simultaneous iodine cell fluorescence measurements. A typical perpendicular Ar I LIF measurement is shown in Figure 6.13, along with the fits used to determine the locations of the iodine fluorescence and LIF peaks. The iodine peak used in this measurement is the one we believe to be at a wavelength of $667.9091 \mathrm{~nm}$. The difference of $0.0035 \mathrm{~nm}$ from the rest frame LIF absorption line at $667.9126 \mathrm{~nm}(2.35$ $\mathrm{GHz}$ ) is then subtracted from the total wavelength difference between the iodine and LIF lines. In the example shown in Figure 6.13, the iodine peak is at $3.09 \mathrm{GHz}$ and the LIF peak is at $0.49 \mathrm{GHz}$, yielding a difference of $2.6 \mathrm{GHz}$ and a net shift in the LIF absorption line of $0.25 \mathrm{GHz} .0 .25 \mathrm{GHz}$ corresponds to a neutral atom velocity of approximately $170 \mathrm{~m} / \mathrm{s}$ toward the laser. If we include the Woo et al. correction to the iodine line wavelengths $(-0.002 \mathrm{~nm})$, the net shift in the LIF absorption line becomes -1.1 $\mathrm{GHz}$; a neutral velocity of approximately $740 \mathrm{~m} / \mathrm{s}$ away from the laser. 


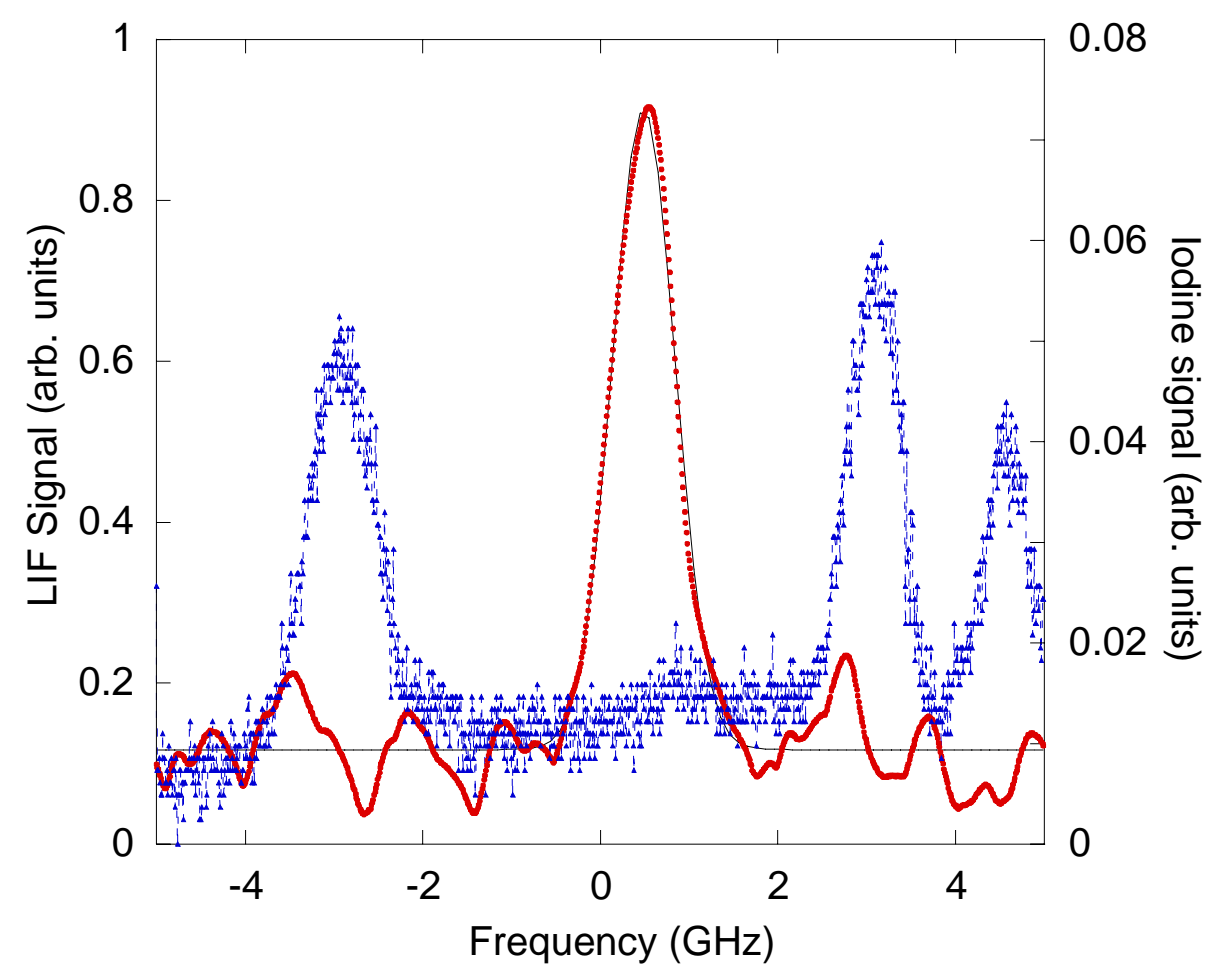

Figure 6.13 Ar I LIF signal (circles, solid line fit) and iodine fluorescence signal (triangles, dashed line fit).

For helicon source parameters of $750 \mathrm{G}$ magnetic field, RF power of $225 \mathrm{~W}$, gas flow of $114 \mathrm{sccm}$, fill pressure of $6.0 \mathrm{mTorr}$, and operating pressure of $13.3 \mathrm{mTorr}$, the radial profile of radial neutral velocities (without the Woo et al. correction) is shown in Figure 6.14. All the measurements are positive and lie within the statistical error of each other. Thus, the neutral flow, if non-zero for these source parameters, is constant across the plasma diameter to within the error in the measurements. Inclusion of the Woo et al. correction would add a uniform correction to all the measured flow speeds yielding a constant flow that is negative (in the opposite direction), but would not change the lack of significant radial variation in the measurements. For a cylindrically symmetric plasma, it seems reasonable to expect that the direction of radial neutral flow, whether inward or outward, should change sign on each "side" of the discharge, i.e., positive and negative 
radius in Figure 6.14. Therefore, non-zero flow across the diameter of the plasma seems to be completely unphysical. Because the use of either of the two different zero-velocity references (ours and the Woo et al. correction) yields an unphysical constant radial velocity across the plasma diameter, these measurements may indicate that both wavelength references are in error and the absolute neutral flow is consistent with zero across the entire plasma diameter.

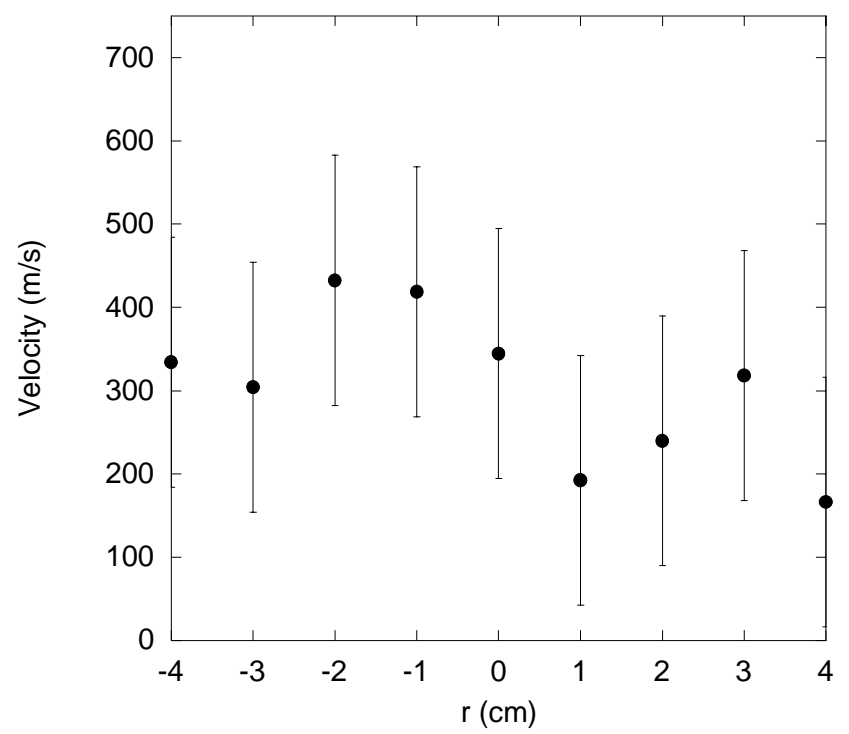

Figure 6.14 Neutral argon radial flow velocity versus radial position.

Radially resolved flow velocity measurements of argon ions were also obtained with the portable LIF diagnostic. The intent was to obtain azimuthal flow measurements of both ions and neutrals at the same plasma parameters to investigate the coupling between ions and neutrals in a rapidly rotating plasma. Unfortunately, due to the low laser power, it was difficult to achieve reasonable signal to noise levels for both species at the same plasma parameters. To increase the signal to noise by avoiding the bright plasma core, an additional mirror used to inject the laser beam into the plasma was 
translated vertically - enabling measurements to be taken at non-zero $y$ values. For measurements taken at $x=0$, the $y$ value will correspond to the radial value, $r$. A few measurements were taken at non-zero $x$ and $y$, so that the $r$ value is given by $\sqrt{x^{2}+y^{2}}$. Because an additional mirror was required to permit vertical translation of the injection mirror, the laser polarization was rotated by $90^{\circ}$ and, instead of pumping the $\pi$ transition with perpendicular laser injection, the two equally spaced (about the center wavelength) circularly polarized $\sigma$ lines were excited. The net shift of the absorption line was determined by locating the center of the two sigma peaks and comparing that wavelength to the reference iodine wavelength. An example of such a LIF measurement is shown in Figure 6.15. 


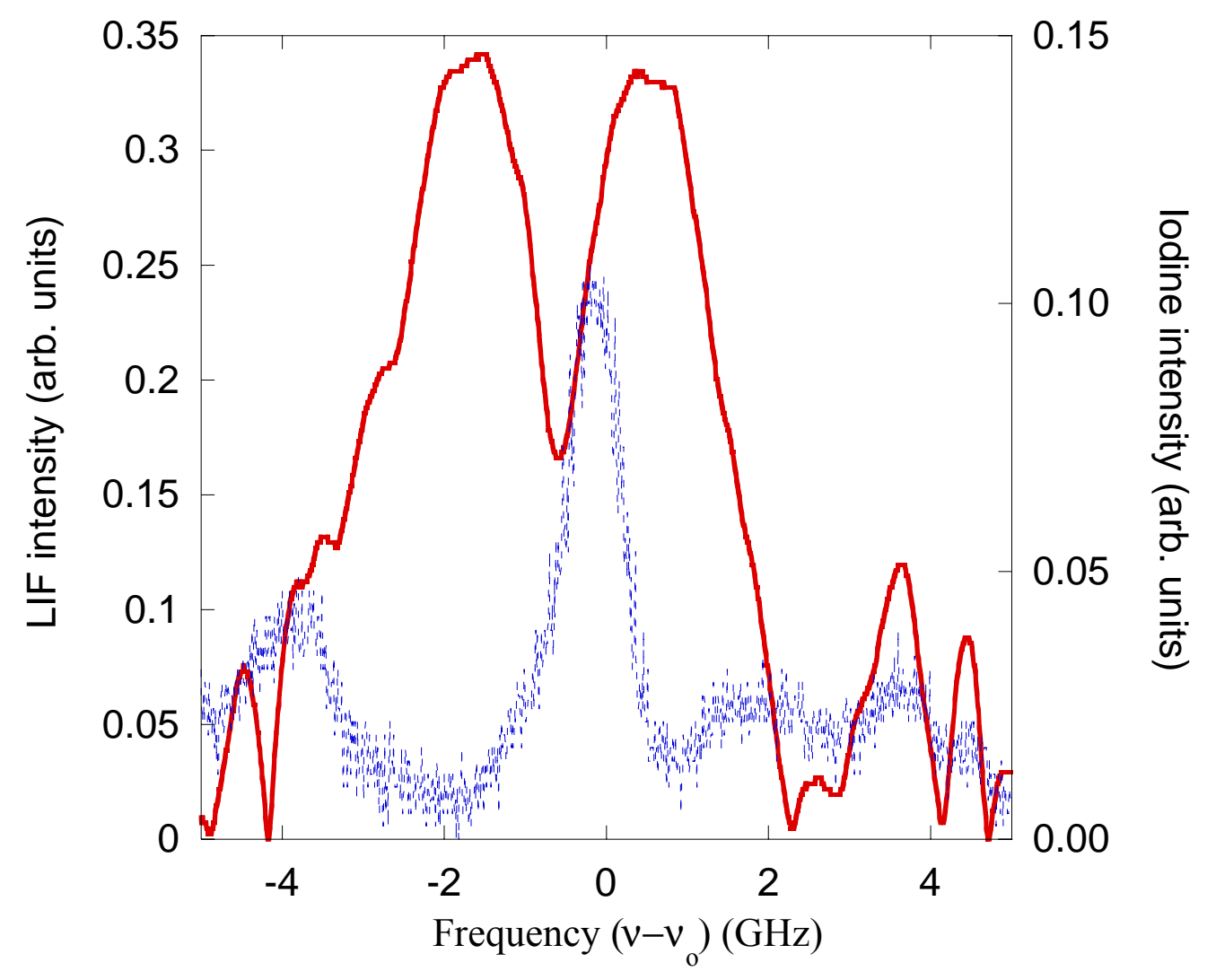

Figure 6.15 LIF signal (solid line) and corresponding iodine signal (dashed line) versus frequency for Ar II flow measurements.

Because the separation of the two sigma peaks depends linearly on the ambient magnetic field strength, the peak spacing also provides an independent measure of the local magnetic field strength. For the Ar II LIF absorption transition, the shift of each sigma peak from the central absorption wavelength will be $\pm 2.22 \times 10^{-3} \mathrm{~nm}$ for a magnetic field of $1 \mathrm{kGauss} .{ }^{2}$ Thus, the separation of the peaks is given by

$$
\Delta P=2\left(2.22 \times 10^{-3} \mathrm{~nm}\right) B / 1000
$$

where $B$ is in Gauss. Since we can determine the separation of the peaks from Figure 6.15, we can solve Equation (6.1) for $B$. The peak locations are at $v=-1.74$ and 0.49 $\mathrm{GHz}$, respectively, giving a peak separation of $2.2 \mathrm{GHz}$, or $3.32 \times 10^{-3} \mathrm{~nm}$. Using 
Equation (6.1), this gives a magnetic field of $747 \pm 7 \mathrm{G}$, in excellent agreement with the applied field strength of $750 \mathrm{G}$.

For helicon source parameters of $750 \mathrm{G}$ magnetic field, RF power of $400 \mathrm{~W}$, gas flow of $100 \mathrm{sccm}$, fill pressure of $5.0 \mathrm{mTorr}$, and operating pressure of $14.9 \mathrm{mTorr}$, the radial profile of perpendicular ion velocities (without the Woo et al. correction) is shown in Figure 6.16. The measurements shown were obtained at $(x, y)=(0,1),(0,2)$, and $(1$, 2). Due to poor signal to noise, measurements at only three spatial locations were obtained. To place these three measurements in context, perpendicular ion flow measurements obtained using dye laser-based LIF for the same plasma parameters and at the same locations ${ }^{3}$ are also presented in Figure 6.16. The diode laser ion flow measurements are in good agreement with the more complete dye laser measurements of the perpendicular ion flow.

While we were unable to directly investigate neutral and ion flows at the same location and for the same plasma conditions with the current version of the portable LIF diagnostic, we have shown that even this low power LIF diagnostic is capable of making both Ar I and Ar II flow measurements. With increased laser power or for more ideal plasma conditions, this diagnostic method might still provide comparative measurements of ion and neutral flows. 


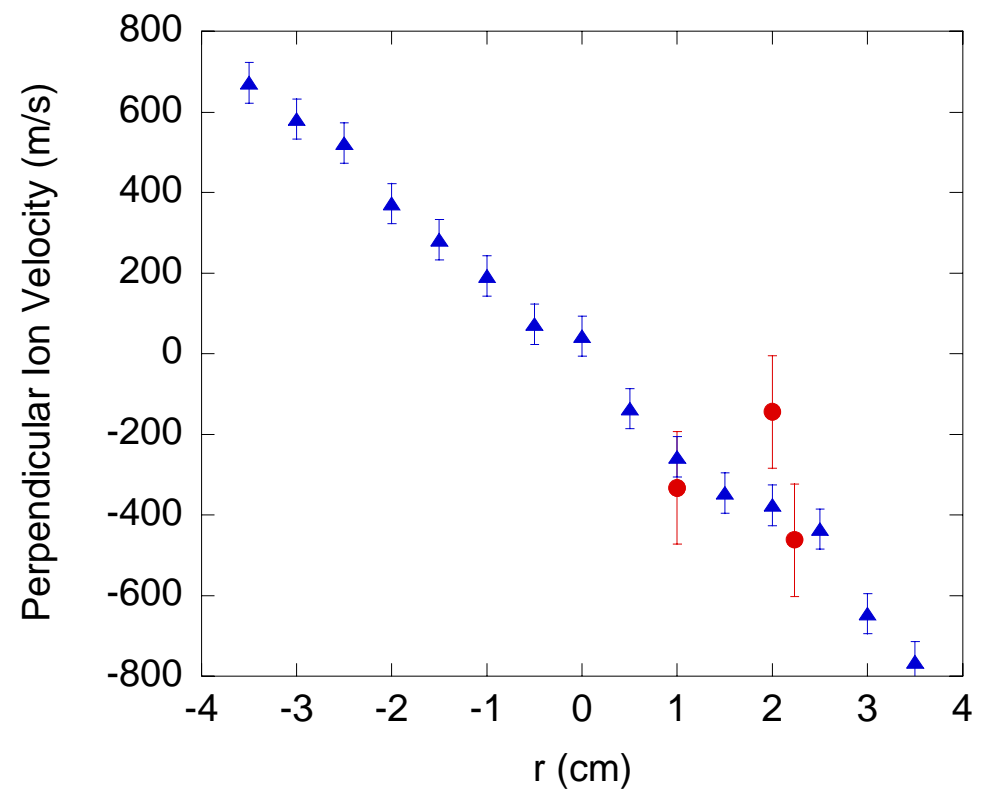

Figure 6.16 Perpendicular ion flow velocity radial profile ${ }^{3}$ obtained using the diode laser (circles) and the dye laser (triangles).

\subsection{Pulsed plasma measurements}

While we usually investigate steady state helicon plasmas with LIF, we recently extended our LIF measurement capabilities to permit time resolved measurements in pulsed helicon plasmas. ${ }^{4}$ The signal to noise levels achievable with the pulsed plasma LIF technique limited us to a capacitive mode case with $750 \mathrm{G}$ magnetic field, RF power of $200 \mathrm{~W}$, gas flow of $114 \mathrm{sccm}$, fill pressure of $6.0 \mathrm{mTorr}$, and operating pressure of 12.5 mTorr. The plasma was pulsed at a frequency of $5 \mathrm{~Hz}$ and the LIF signal for radial injection of the laser was averaged over 150 pulses for each wavelength (frequency) step using the method described in Ref. 4. Three-dimensional surface plots of LIF signal amplitude versus laser frequency (shift from the absorption line) and time are shown in 
Figure 6.17. The background light (LIF signal level when the laser is not near absorption wavelength) throughout the pulse was subtracted from the total signal at each wavelength. Throughout most of the pulse, significant LIF signal was obtained. An oblique view, a time-axis view, and a top view are provided in Figure 6.17. The plasma pulse begins at $0.02 \mathrm{~s}$ and terminates at $0.12 \mathrm{~s}$. The lock-in amplifier integration time constant of $10 \mathrm{~ms}$ used for these measurements limits the time resolution of the measurement to roughly $50 \mathrm{~ms}$. Therefore, the delay in the initial signal response and the slow decay of the signal at the end of the pulse are artifacts of the lock-in time constant used. Such long lock-in time constants were required to obtain useful signal-to-noise levels in the experiment. It is possible to numerically filter the LIF data and slightly improve the time resolution of the time resolved data. ${ }^{5}$ However, because these measurements were undertaken simply as a proof-of-principle demonstration, such improvements of the time resolution of the data were not performed.

The LIF signal amplitude increases throughout the pulse. Without comparing the measurements to $\mathrm{CR}$ model results based on measured plasma density and electron temperature behaviors throughout the pulse (not available for these discharges), it is not possible to determine if the ground state neutral density increased or decreased throughout the pulse. To achieve the goal of measuring the evolution of the ionization fraction throughout the pulse, time resolved measurements of the plasma characteristics (density and electron temperature) and edge neutral pressure are required. At the moment, we do not have a means of obtaining the time resolved edge neutral pressure measurements necessary to perform the CR modeling for the entire pulse. 

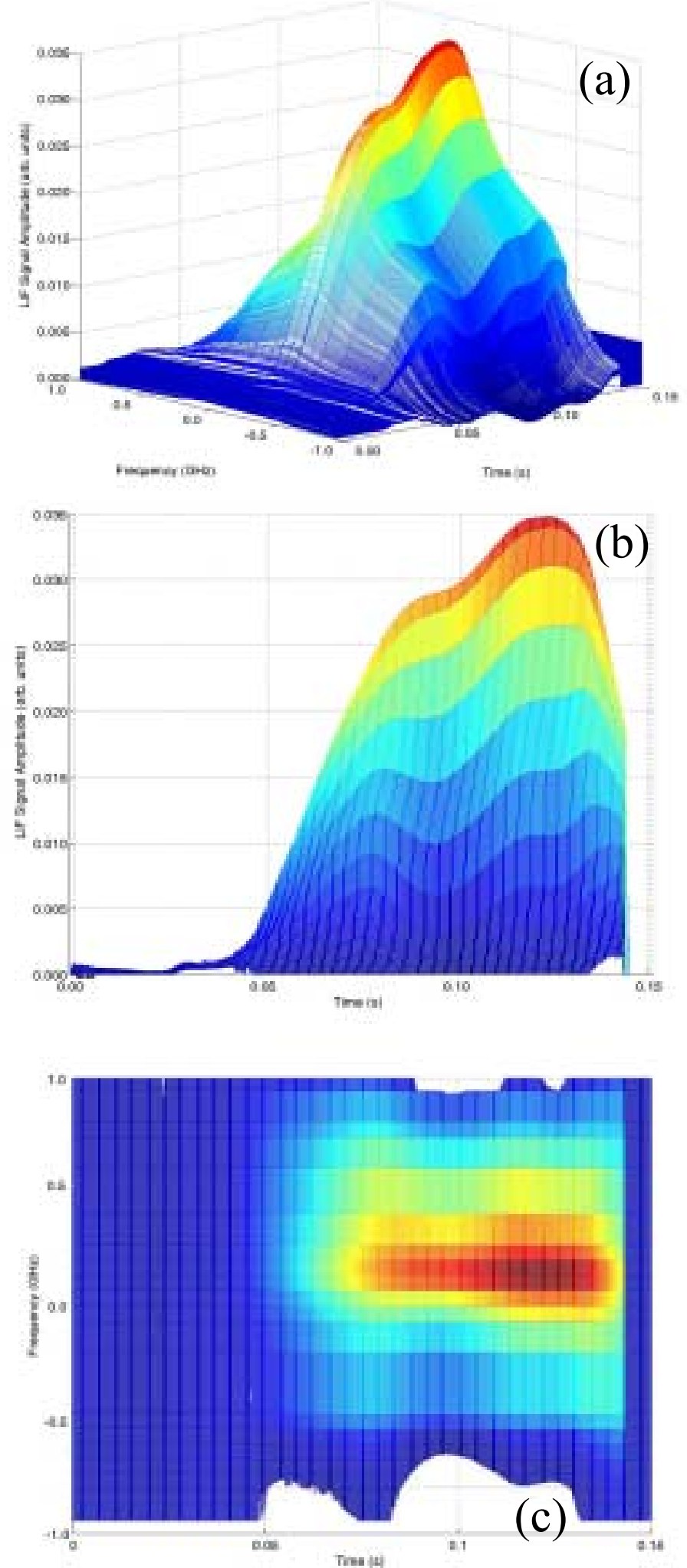

Figure 6.17 LIF Signal amplitude versus laser frequency and time in a) oblique, b) time axis, and c) top views at $r=2.0 \mathrm{~cm}$. 


\section{References}

${ }^{1}$ J. M. Hanna, An Investigation Of Neutral Effects On Ion Cyclotron Wave Propagation In The Auburn Linear Experiment For Space Plasma Investigations, Ph.D. Dissertation, Auburn University, Auburn (2003).

${ }^{2}$ R.F. Boivin, "Zeeman Splitting for LIF Transitions and De-convolution Technique to Extract Ion Temperature," PL-050, EPAPS-E-PHPAEN-10-003306 (2001).

${ }^{3}$ R. Hardin, X. Sun, and E. Scime, Rev. Sci. Instrum. 75, 4103 (2004).

${ }^{4}$ E. Scime, C. Biloiu, C. Compton, F. Doss, J. Heard, E. Choueri, S. Spektor, Rev. Sci. Instrum. 76, 026107 (2005).

${ }^{5}$ G. Jackson, C. Lewis, S. Doorn, V. Majidi, F. King, Spectrochimica Acta B 56, 2449 (2001). 


\section{Chapter 7: Comparison of CR Model and Measurements}

As noted previously, the LIF and emission spectroscopy spatial profile measurements shown in Chapter 6 are of excited neutral states. To interpret those measurements in terms of the ground state spatial profile, the predictions of the CR model for the excited state spatial profile were compared to the experimental measurements. The electron temperature measurements were used to create Maxwellian EEDFs with total densities given by the measured plasma density profile for input into the model along with the neutral fill pressure at the edge of the plasma (according to the Baratron gauge measurements obtained with the discharge off). The CR model was then executed for an initial guess for the ground state density and the normalized measured excited state profile (the peak value of the measurements scaled to the peak value of the CR model for consistency in units) compared to the predictions of the CR model. The parameters describing the ground state profile, as well as the presence and details of an electron beam in the EEDF, were varied until the best match between the measurements and the model was obtained. Because the optical diagnostics average over a finite volume $(\sim 0.5 \mathrm{~cm})$ of plasma, whereas the probe measurements are localized to less than $2 \mathrm{~mm}$, the CR model results shown here have been smoothed with a running window (effective width approximately $0.75 \mathrm{~cm}$ ). The density profile of State 3 was the most sensitive to changes in the model input parameters because it was the lowest energy state examined and it is closer in energy to the heavily populated metastable states than States 10 and 11. Thus, the radial structure in the measured plasma properties, e.g., electron temperature, is most evident in the CR model generated profiles for State 3. Comparison of the LIF measurement of the State 3 density profile to the CR model results for the State 3 density 
profile was the primary method of determining the optimal neutral density profile to use in the CR model. Comparison of the emission spectroscopy measurements to the $\mathrm{CR}$ predictions for States 10 and 11 were then used as additional checks of the model output. Quantification of the goodness of fit of the CR predictions with the measurements was accomplished with a chi square test between the two profiles,

$$
\chi^{2}=\frac{1}{N} \sum_{i=1}^{N} \frac{\left(L\left(r_{i}\right)-M\left(r_{i}\right)\right)^{2}}{L\left(r_{i}\right) M\left(r_{i}\right)},
$$

where $L\left(r_{i}\right)$ is the measurement at radial position $r_{i}, M\left(r_{i}\right)$ is the CR model density value at radial position $r_{i}$, and $N$ is the number of radial positions measured.

\subsection{Parameter Set A (6 mTorr Helicon mode)}

The best match for the 6 mTorr helicon mode plasma case required a ground state profile with a maximum $60 \%$ depletion and a depletion width of $3 \mathrm{~cm}(P=0.6$ and $W=$ 3.0, respectively) and Maxwellian EEDFs with no electron beam. Depletion levels of $55 \%$ to $65 \%$ were reasonably consistent with the measurements, but significantly different depletion levels (50\% or $70 \%)$ yielded State 3 profiles that were not consistent with the measured radial profile. Comparisons of the CR model predictions and the LIF and emission measurements are shown in Figure 7.1. The chi-square test for State 3 yielded 0.05 . The rise in the CR model prediction for State 3, State 10, and State 11 at the edge of the plasma $(r>4 \mathrm{~cm})$ results from the increase in measured electron temperature at the plasma edge (see Figure 6.1b). 

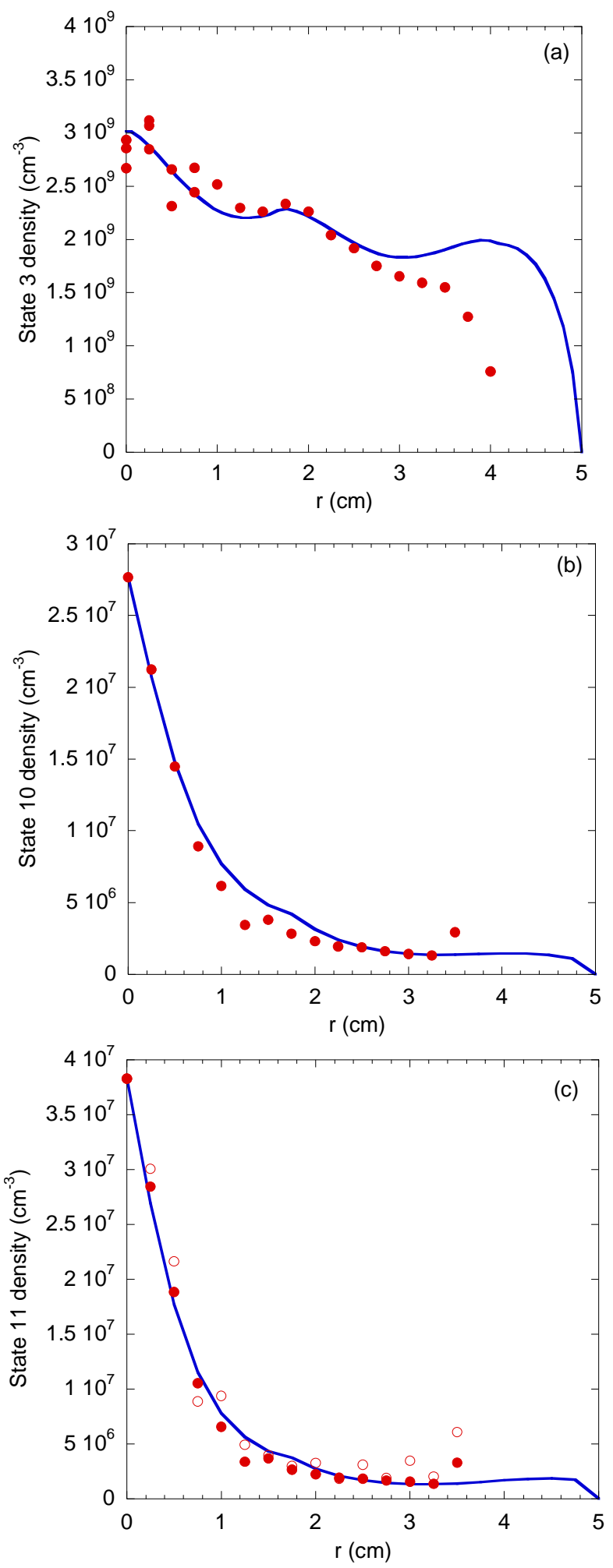

Figure 7.1 Neutral argon excited state density versus radial position, comparison between experimental data (circles) and CR model output (solid line) for a $3 \mathrm{~cm}$ wide depletion of $60 \%$. a) State 3. b) State 10. c) State 11. In (c) the nearly identical measured profiles for the $667.9 \mathrm{~nm}$ (open circles) and $750.6 \mathrm{~nm}$ (filled circles) transitions are shown. 
Note that the LIF intensity (State 3 density) varies by only slightly more than a factor of two across the plasma radius, while the Abel inverted emission data (State 10 and State 11 densities) vary by more than an order of magnitude. The key result of this analysis is that the almost flat LIF intensity profile and the Abel inverted emission profile data are both consistent with a hollow ground state neutral profile given the measured electron density and temperature profiles. This conclusion is completely dependent on the availability of the CR model results. The best fit neutral ground state profile for the helicon mode case is shown in Figure 7.2. A flat neutral density profile yields state density profiles that are inconsistent with the LIF and the emission profile measurements. Therefore, this analysis confirms that the neutral depletion on-axis is significant for relatively high pressure argon helicon discharges.

Because the CR modeling indicates an on-axis depletion, the on-axis ionization fraction can be determined more accurately than if only the edge neutral pressure is known. The ionization fraction is given by $N_{e} / N_{n}$. Using the Langmuir probe measurement of the electron density on-axis and the neutral density on-axis as determined by the $\mathrm{CR}$ modeling, the plasma is $28 \%$ ionized. If only the edge neutral density were known, one would mistakenly calculate the ionization to be $11 \%$. 


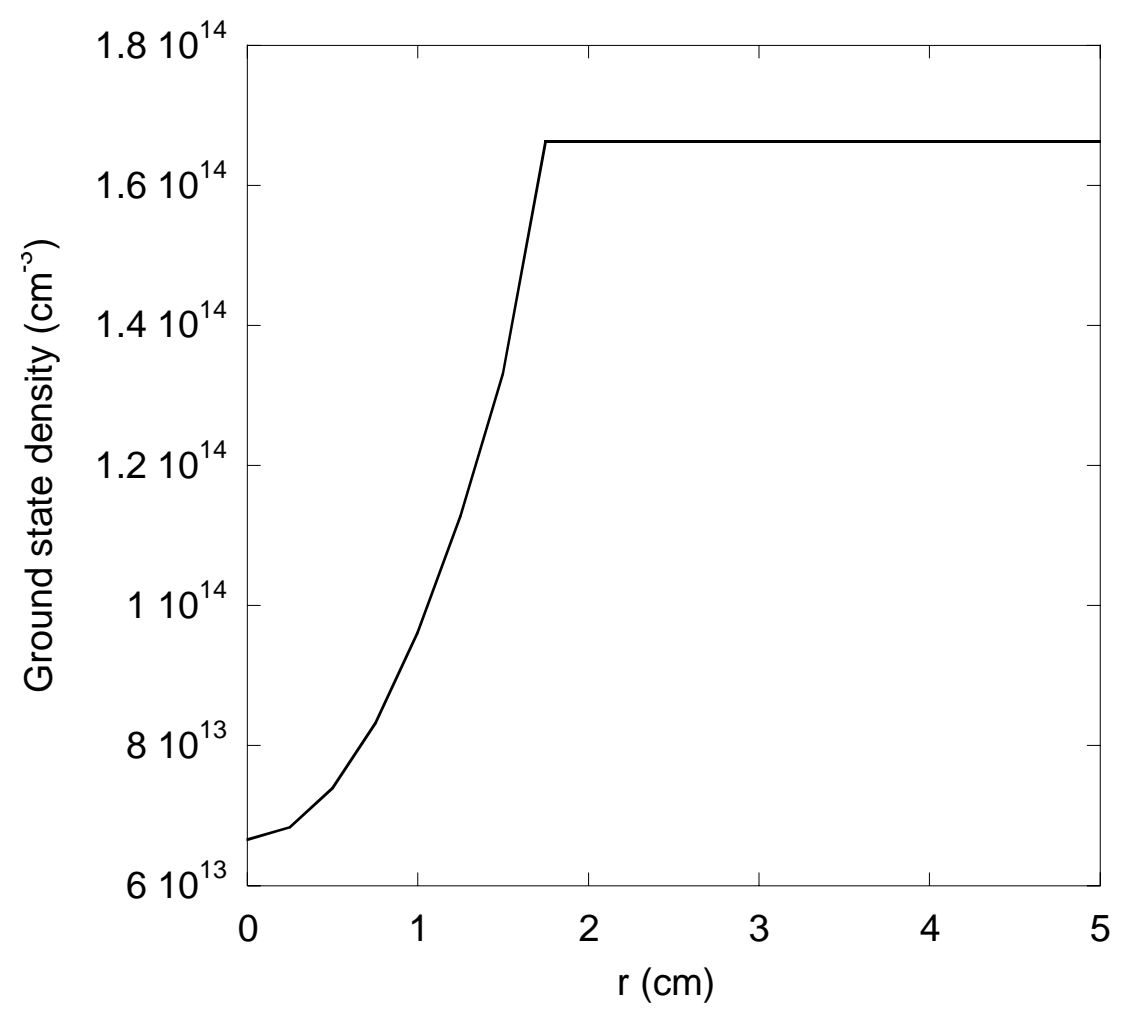

Figure 7.2 Ground state density profile for $P=0.6$ and $W=3 \mathrm{~cm}$.

\subsection{Parameter Set B (Capacitive mode)}

The strong asymmetries in the measured profiles made analysis of the capacitive mode plasma case considerably more difficult. Note that for the capacitive case, the variation in the LIF intensity across the plasma radius is roughly a factor of four, whereas the Abel inverted emission measurements vary by only a factor of two. Without further analysis, it is already clear that the spatial distribution of neutrals in this source configuration is likely to be quite different from the helicon case.

Since the peaks in the electron temperature and the emission data occurred offaxis (see Figure 6.7), the measurements and the CR model predictions were compared for two different normalization methods: normalization to the values at $r=0$ and also to the 
peak values observed. The initial ground state profile guess used was a depletion of $20 \%$ and a depletion width of $5 \mathrm{~cm}$ (Figure 7.3a). The chi-square test for this case yielded 0.26 . The best match for the $r>0$ data (the region over which the plasma density and electron temperature measurements were available) was for a flat ground state profile with normalization at $r=0$ (Figure 7.3b). The chi-square test yielded 0.07 . The poor fit results are dominated by the CR model peak caused by the off-axis peak in the electron temperature profile. In other words, the peak in electron temperature at the edge of the plasma forces a CR model prediction for State 3 that is much flatter in the core and extends to larger radii than what is observed experimentally.
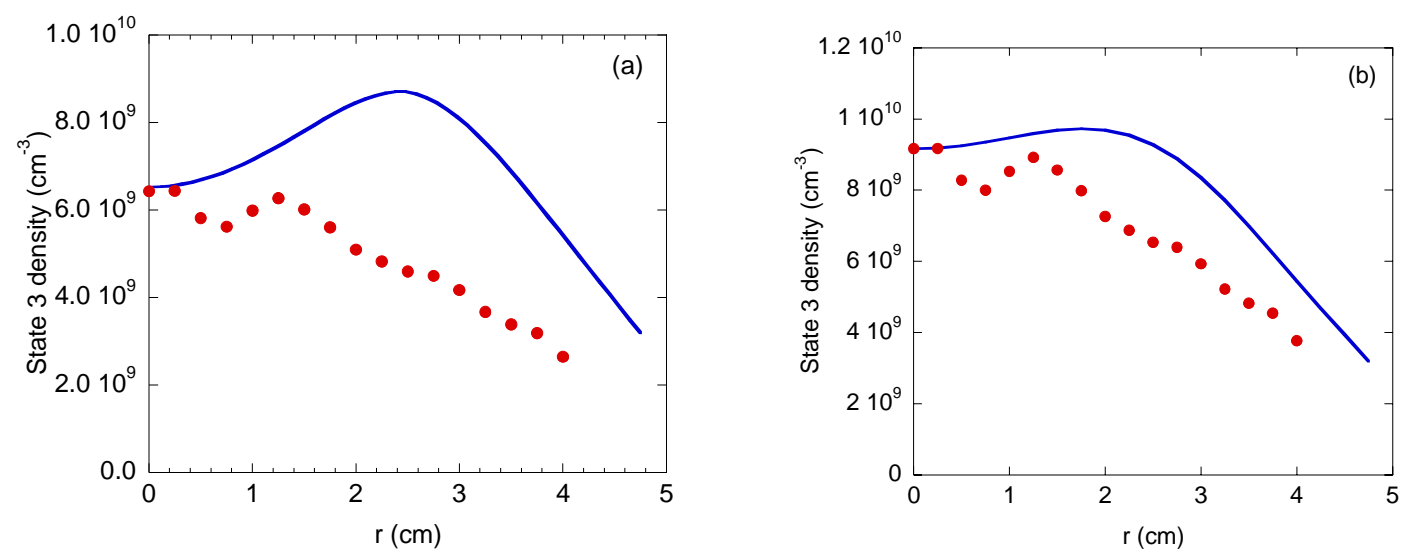

Figure 7.3 Neutral argon State 3 density versus radial position, comparison between experimental data (circles) and CR model output (solid line) with model parameters of a) $P=0.2, W=5.0$ and b) constant ground state density.

The corresponding comparisons of the Abel inverted emission measurements and the CR model predictions are shown in Figure 7.4. Due to the poor signal-to-noise for the $667.9 \mathrm{~nm}$ emission measurements (see Figure 6.8a), only the comparisons using the 750.6 $\mathrm{nm}$ transition results are shown. The chi square tests associated with Figure 7.4 are a) 
0.02, b) 0.03 , c) 0.07 , and d) 0.09 . A $5 \mathrm{~cm}$ wide, $20 \%$ depletion neutral profile (Figure 7.4a and Figure 7.4b) yields a CR model prediction more consistent with the emission measurements than a flat neutral profile, particularly for State 11 (Figure 7.4c and Figure 7.4d). However, the fairly poor agreement between the LIF measurements and the CR model suggest that for these source parameters, the model neutral density profile function may not have enough structure to accurately represent the actual ground state neutral profile.
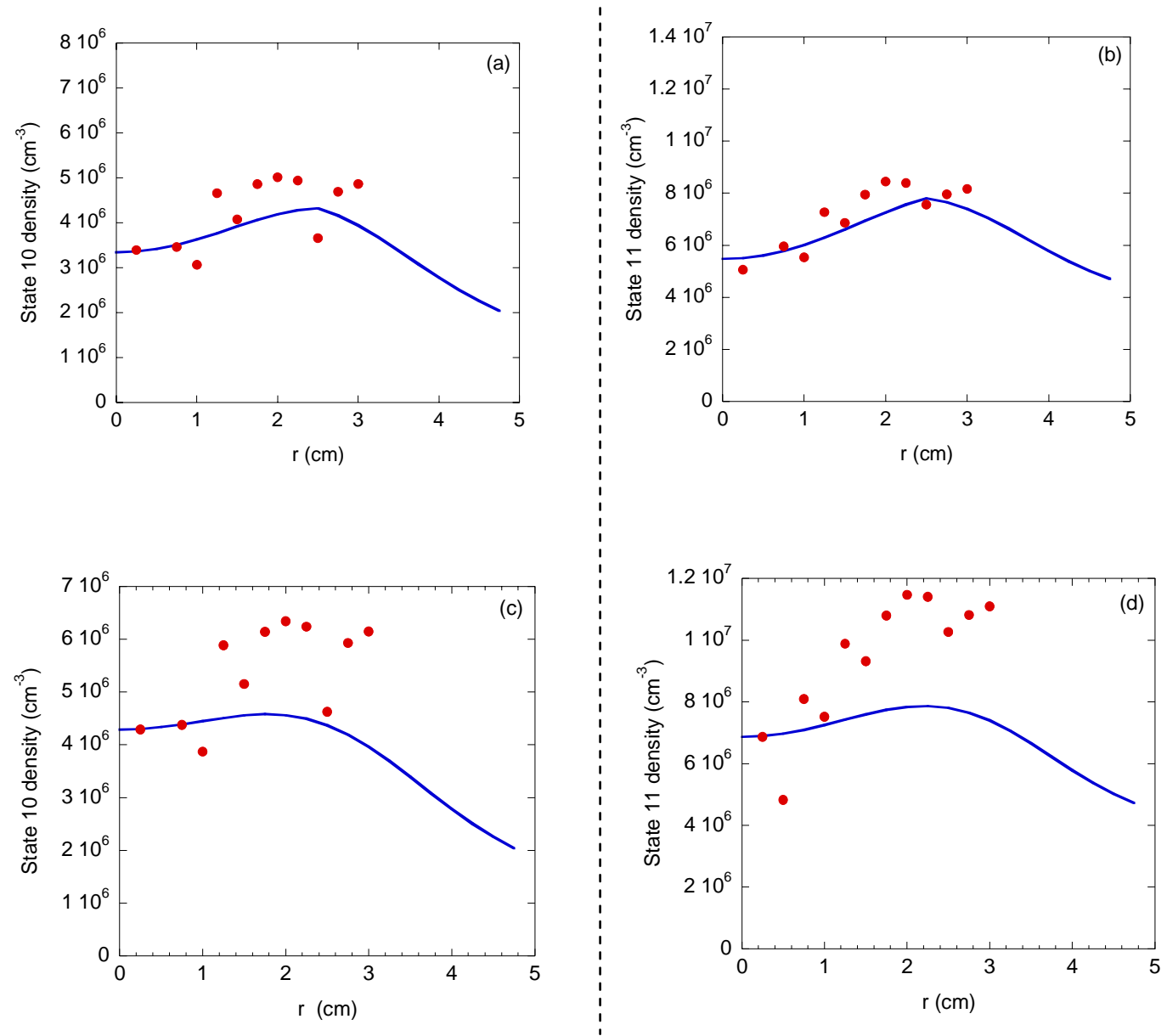

Figure 7.4 Neutral argon excited state density versus radial position, comparison between experimental data (circles) and CR model output (solid line) for a) and b) $P=0.2, W=5.0$ and c) and d) constant ground state density. State 10 is shown in a) and c) and State 11 is shown in b) and d). 
Because the discharge is capacitive and has prominent off-axis peaks in the electron temperature, it is reasonable to assume that there may be off-axis depletions of the neutrals. Thus, a ground state neutral profile described by Equation 4.18 was also considered. Such a ground state profile yielded a State 3 density profile that included both off-axis and on-axis peaks. Comparisons for several variations of the parameters $P$, $W$, and $C$ are shown in Figure 7.5. The chi-square test values for the comparisons are a) 0.078 , b) 0.079 , c) $0.080 \mathrm{~d}) 0.085$. Although the modified CR model produces a double peak in the State 3 density profile, the proper spacing between the peaks and the linear decrease from $r=2-4 \mathrm{~cm}$ in the LIF data still could not be reproduced by modifying the magnitude and width of the off-axis depletion in the ground state neutral profile. 

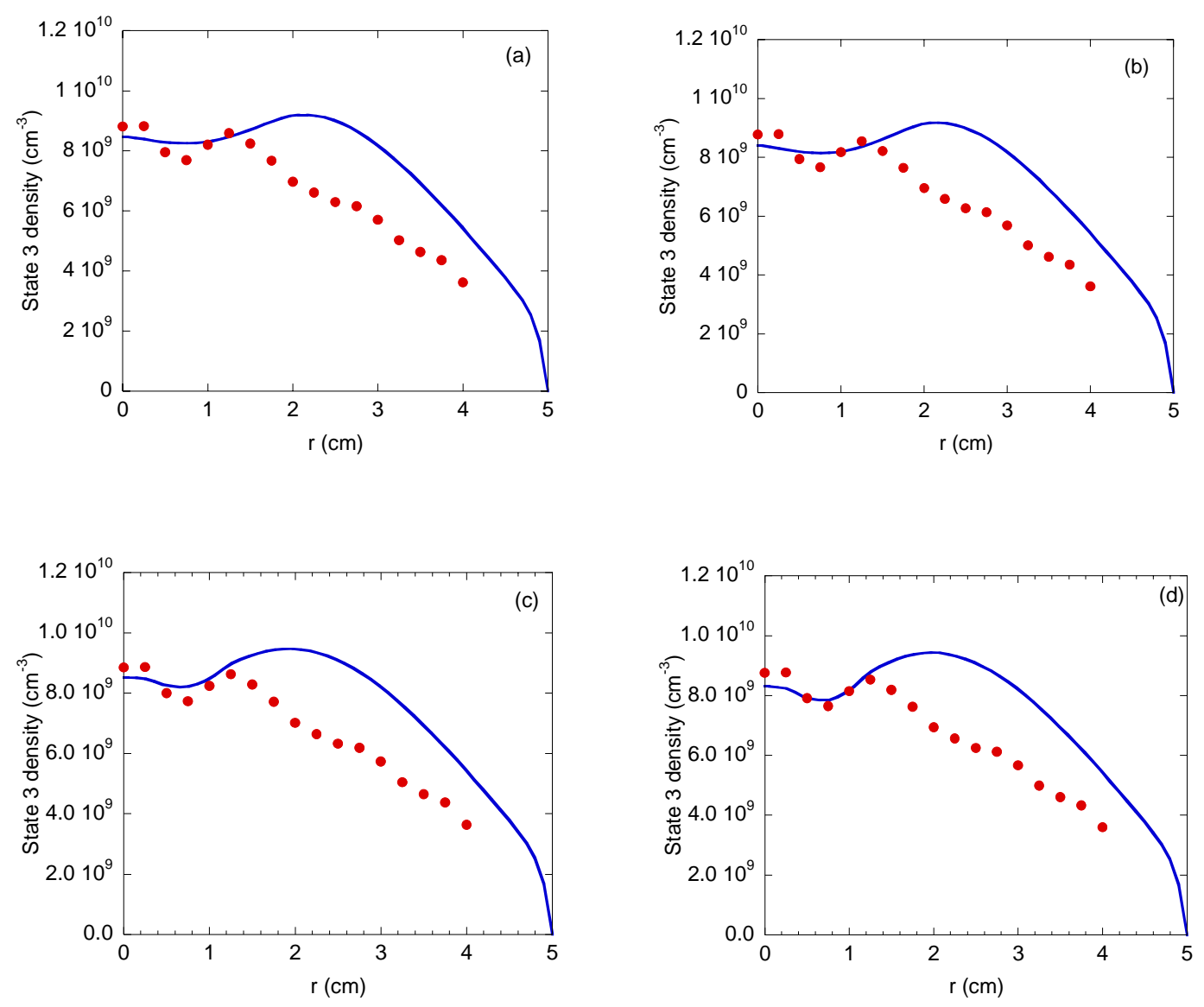

Figure 7.5 Neutral argon State 3 density versus radial position, comparison between experimental data (circles) and CR model output (solid line) with model parameters of a) $P=0.1, W=2.0, C=1.0$, b) $P=$ $0.11, W=2.0, C=1.0$, c) $P=0.15, W=1.0, C=0.75$, and d) $=0.2, W=1.0, C=0.75$. The LIF data are normalized to the model at $r=0$.

Comparisons between the $\mathrm{CR}$ predictions and the emission spectroscopy measurements for $10 \%, 15 \%$, and $20 \%$ off-axis neutral depletions are shown in Figure 7.6. The chi-square values for Figure 7.6 are a) 0.08 , b) 0.06 , c) 0.06 , d) 0.09 e) 0.06 , and 

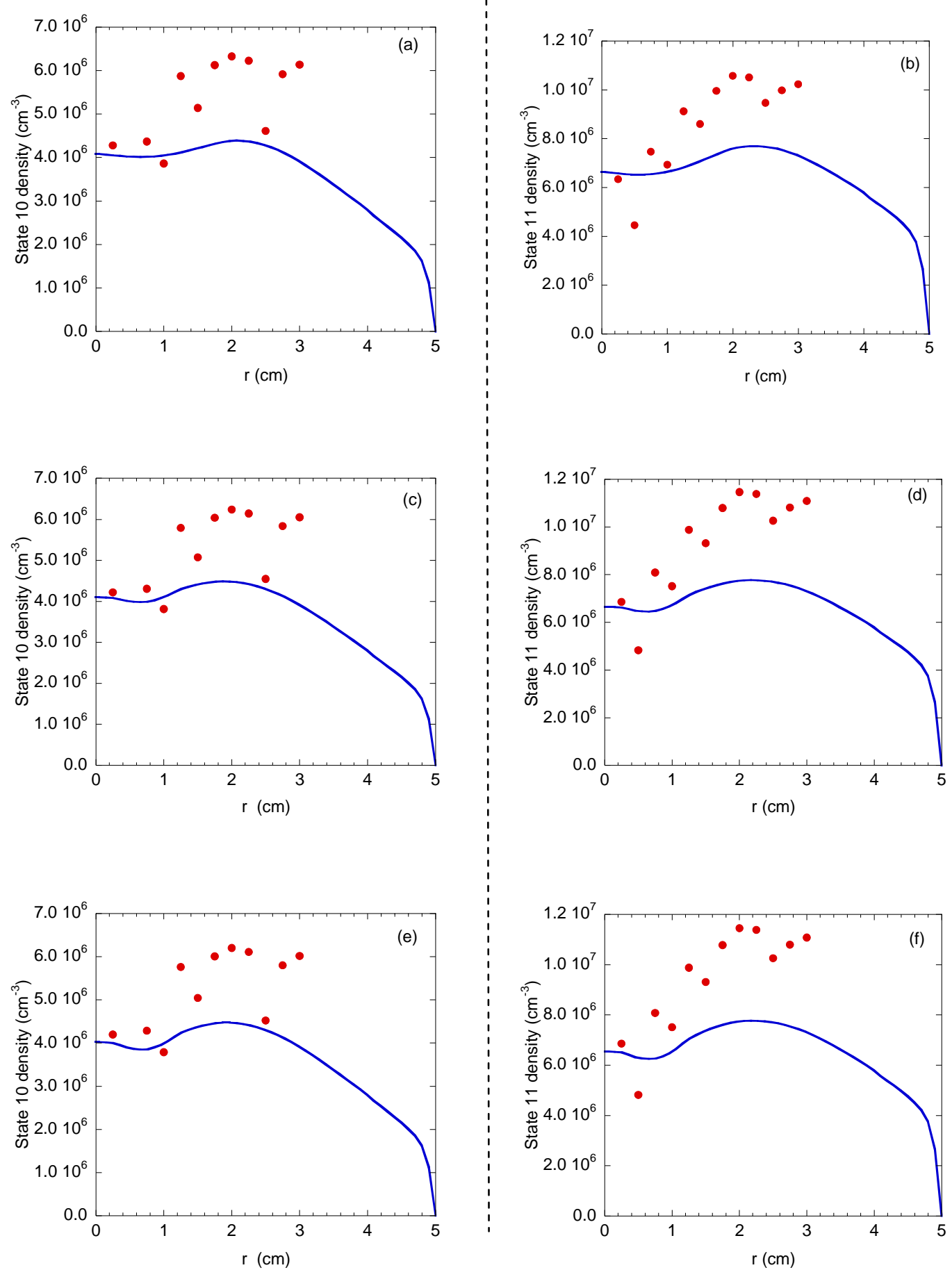

Figure 7.6 Neutral argon excited state density versus radial position, comparison between experimental data (circles) and CR model output (solid line) for a) and b) $P=0.1, W=2.0, C=1.0$ and c) and d) $P=$ $0.15, W=1.0, C=0.75$ and e) and f) $P=0.2, W=1.0, C=0.75$. State 10 is shown in a), c), and e) and State 11 is shown in b), d), and f). The data are normalized to the code at $r=0$. 

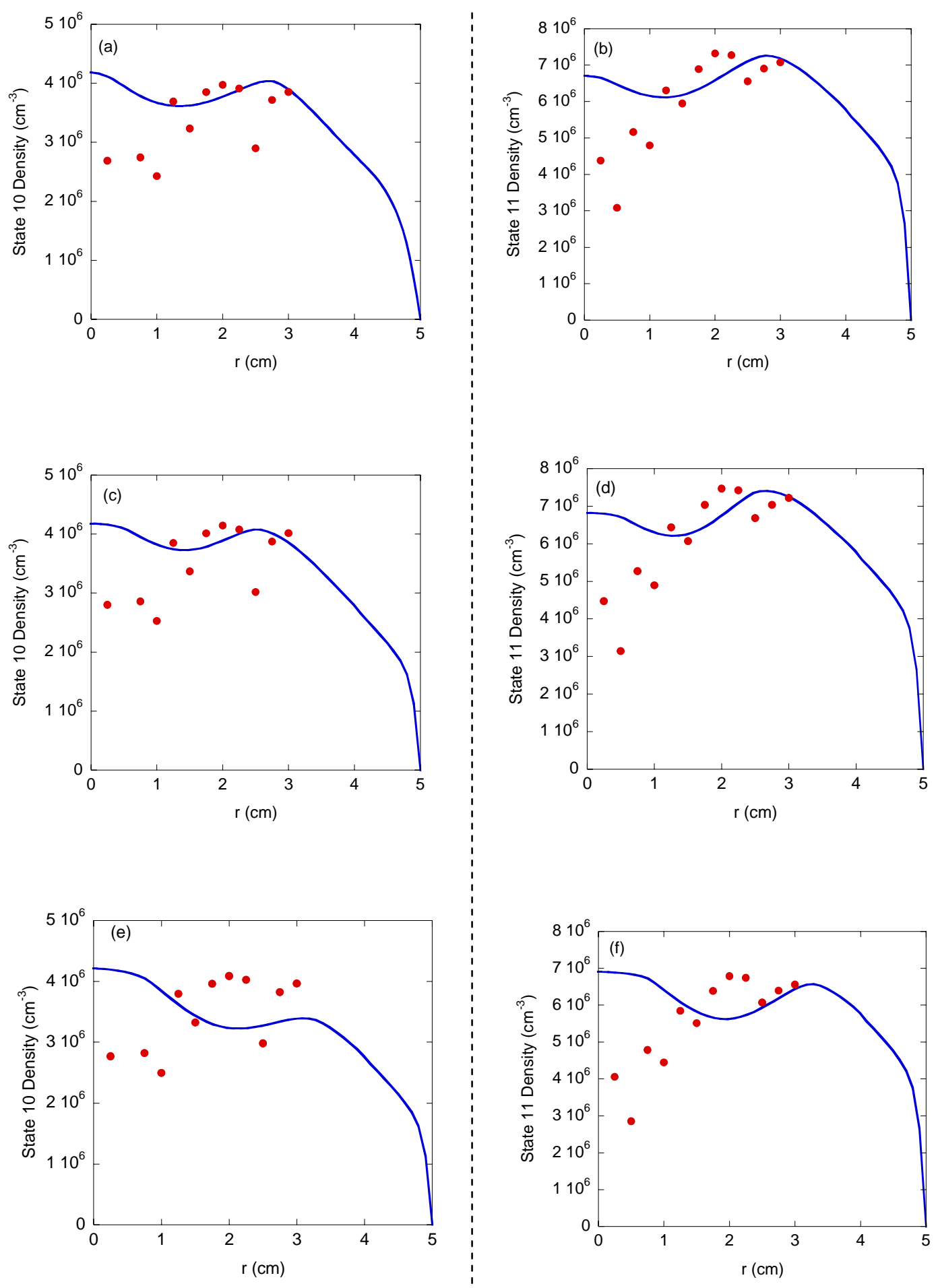

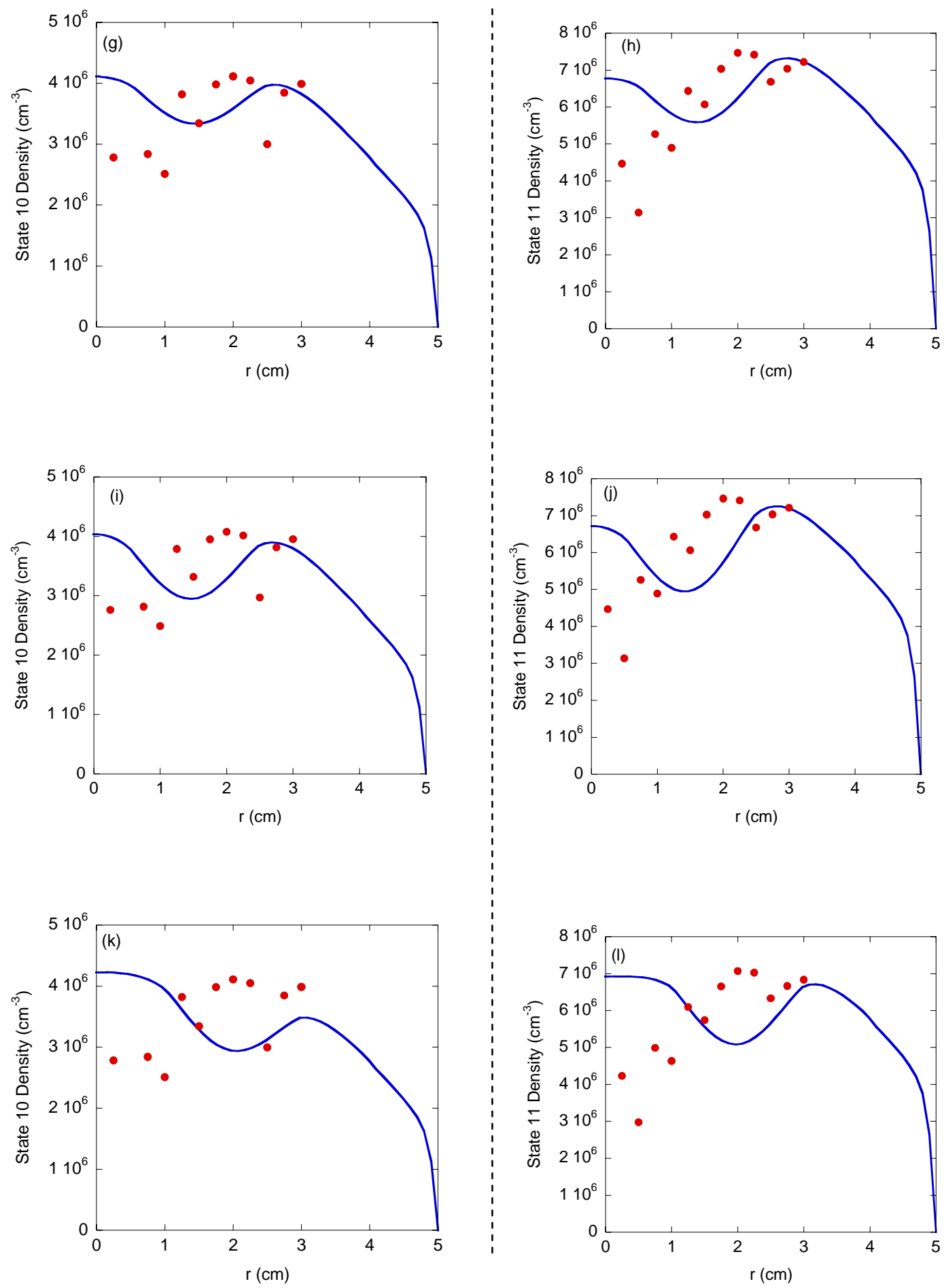

Figure 7.7 Neutral argon excited state density versus radial position, comparison between experimental data (circles) and $\mathrm{CR}$ model output (solid line) for a) and b) $P=0.2, W=2.5, C=1.5$, c) and d) $P=0.2, W$ $=2.0, C=1.5$, e) and f) $P=0.3, W=2.5, C=2.0, \mathrm{~g})$ and h) $P=0.3, W=2.0, C=1.5$, i) and j) $P=0.4, W=$ 2.0, $C=1.5$, and k) and l) $P=0.4, W=2.0, C=2.0$. State 10 is shown in a), c), e), g), i), and k), and State 11 is shown in b), d), f), h), j), and l). The data are normalized to the peak value. 
f) 0.09 . The CR model predictions are less consistent with the data than the single, onaxis depletion model results shown in Figure 7.4a and Figure 7.4b for State 10 and State 11, respectively. Additional ground state neutral profiles were considered and the results are shown in Figure 7.7. The chi-square values for Figure 7.7 are a) 0.06, b) 0.08 , c) 0.05 , d) 0.08 , e) 0.07 , f) 0.13, g) 0.05 , h) 0.09 , i) 0.06, j) 0.11 , k) 0.10 , and l) 0.15

What is clear from the analysis shown in Figure 7.5, Figure 7.6, and Figure 7.7 is that a single, off-axis neutral depletion is inconsistent with the LIF and the emission spectroscopy data. The comparison shown in Figure 7.4 suggests that a central neutral depletion of $20 \%$ does a reasonably good job of reproducing the Abel inverted emission data, but such a neutral density profile does a poor job of reproducing the finer radial structure in the LIF data (which was better fit with an off-axis neutral depletion).

The next possibility considered was the presence of a spatially localized (on-axis), energetic electron beam along with an off-axis neutral depletion. A population of electrons has been measured in a GEC cell run in capacitive mode. ${ }^{1}$ As noted previously, the difference in energy between the excited states probed with LIF and the emission spectroscopy could lead to significant differences in state distributions in the presence of a population of energetic electrons. Comparisons between the CR predictions with an electron beam and the LIF data are shown in Figure 7.8. The associated chi square test results are a) 0.02, b) 0.02 , and c) 0.01 . The respective comparisons between the $\mathrm{CR}$ model predictions and the emission spectroscopy measurements are shown in Figure 7.9. The associated chi square test results are a) 0.21, b) 0.25 , c) 0.18 , d) 0.25 , e) 0.23 , and f) 0.31. While the CR model finally is able to reproduce the measured State 3 profile, the agreement between the CR model and emission data is quite poor. The key feature in 
these data is that the State 3 profile from the LIF measurements is somewhat peaked on axis, while the Abel inverted emission data yields hollow profiles for State 10 and State 11 - a very different situation than the measurements for the high power helicon mode case.
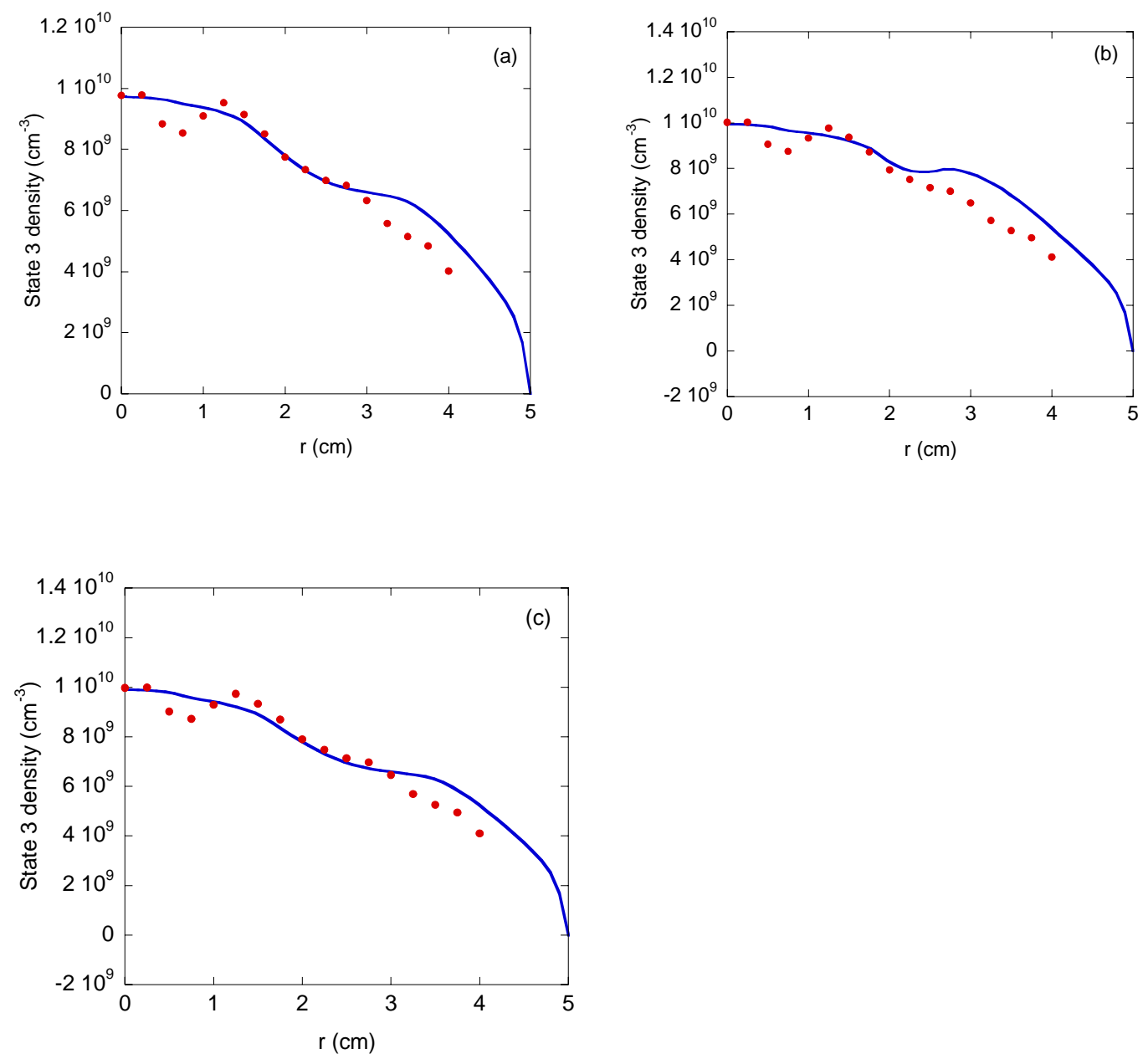

Figure 7.8 Neutral argon State 3 density versus radial position, comparison between experimental data (circles) and CR model output (solid line) with model parameters of a) $P=0.2, W=2.0, C=2.5$ and $20 \mathrm{eV}$ electron beam for $-0.5<r<0.5$, b) $P=0.2, W=1.0, C=2.25$ and $40 \mathrm{eV}$ electron beam for $-0.5<r<0.5$, and c) $P=0.2, W=2.0, C=2.25$ and $40 \mathrm{eV}$ electron beam for $-0.5<r<0.5$. The LIF data are normalized to the model at $r=0$. 

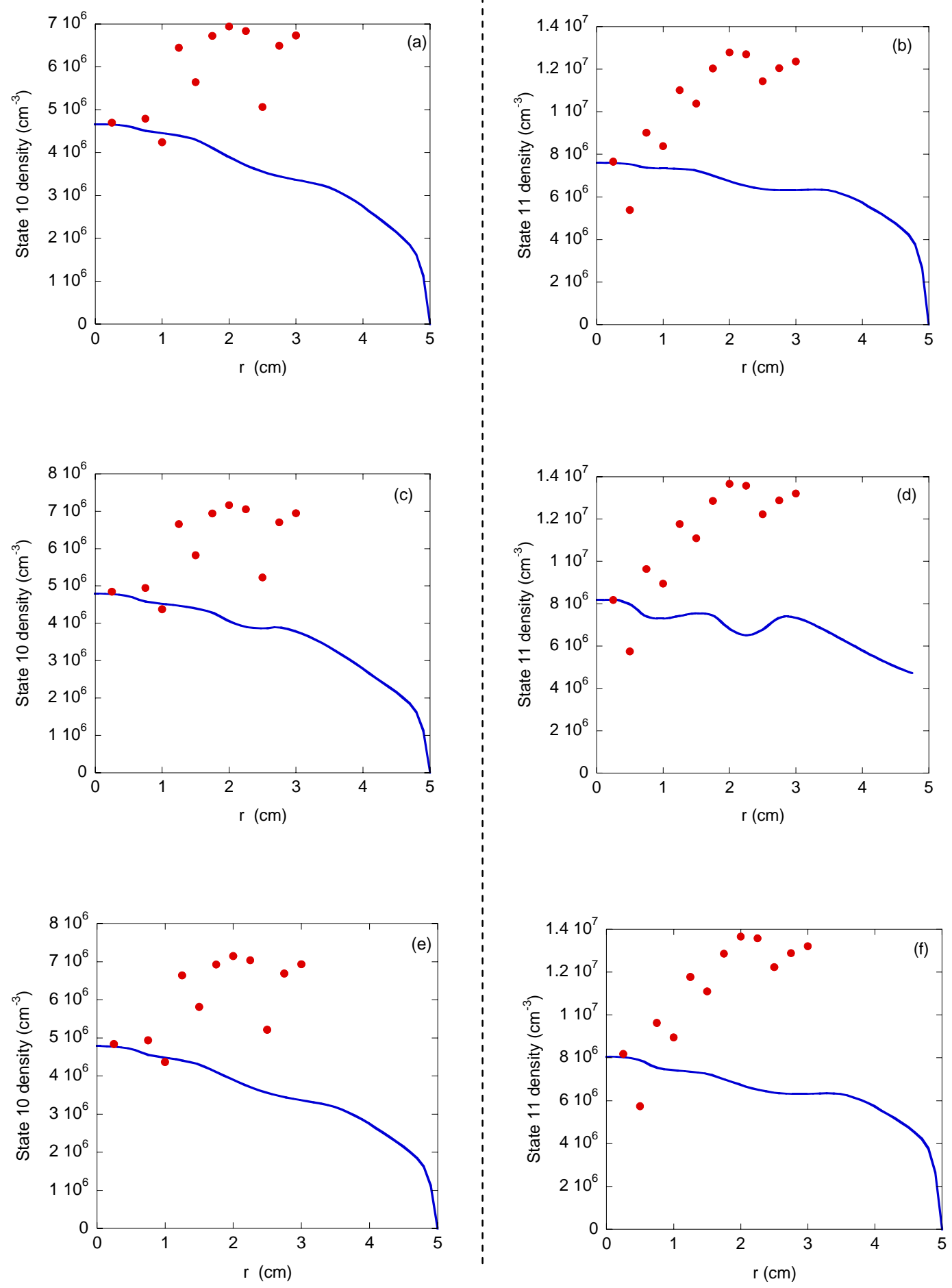

Figure 7.9 Neutral argon excited state density versus radial position, comparison between experimental data (circles) and CR model output (solid line) with model parameters of a) and b) $P=0.2, W=2.0, C=$ 2.5 and $20 \mathrm{eV}$ electron beam for $-0.5<r<0.5, \mathrm{c})$ and d) $P=0.2, W=1.0, C=2.25$ and $40 \mathrm{eV}$ electron beam for $-0.5<r<0.5$, and e) and f) $P=0.2, W=2.0, C=2.25$ and $40 \mathrm{eV}$ electron beam for $-0.5<r<0.5$. State 10 is shown in a), c), and e) and State 11 is shown in b), d), and f). The data are normalized to the model at $r=0$. 
The next CR model considered included a spatially localized (on-axis) energetic electron beam with an on-axis neutral depletion. The objective was to combine the features that seemed to do the best job in reproducing the measured excited state profiles . The comparisons between the LIF measurements and the predicted state profiles from the CR model are shown in Figure 7.10. The corresponding comparisons between the emission spectroscopy measurements and the CR model predictions are shown in Figure 7.11 (only State 11 is shown for simplicity). In this case, the parameters that yield CR model profiles that best fit the measurements for State 3 do a poor job of reproducing the State 11 measurements.
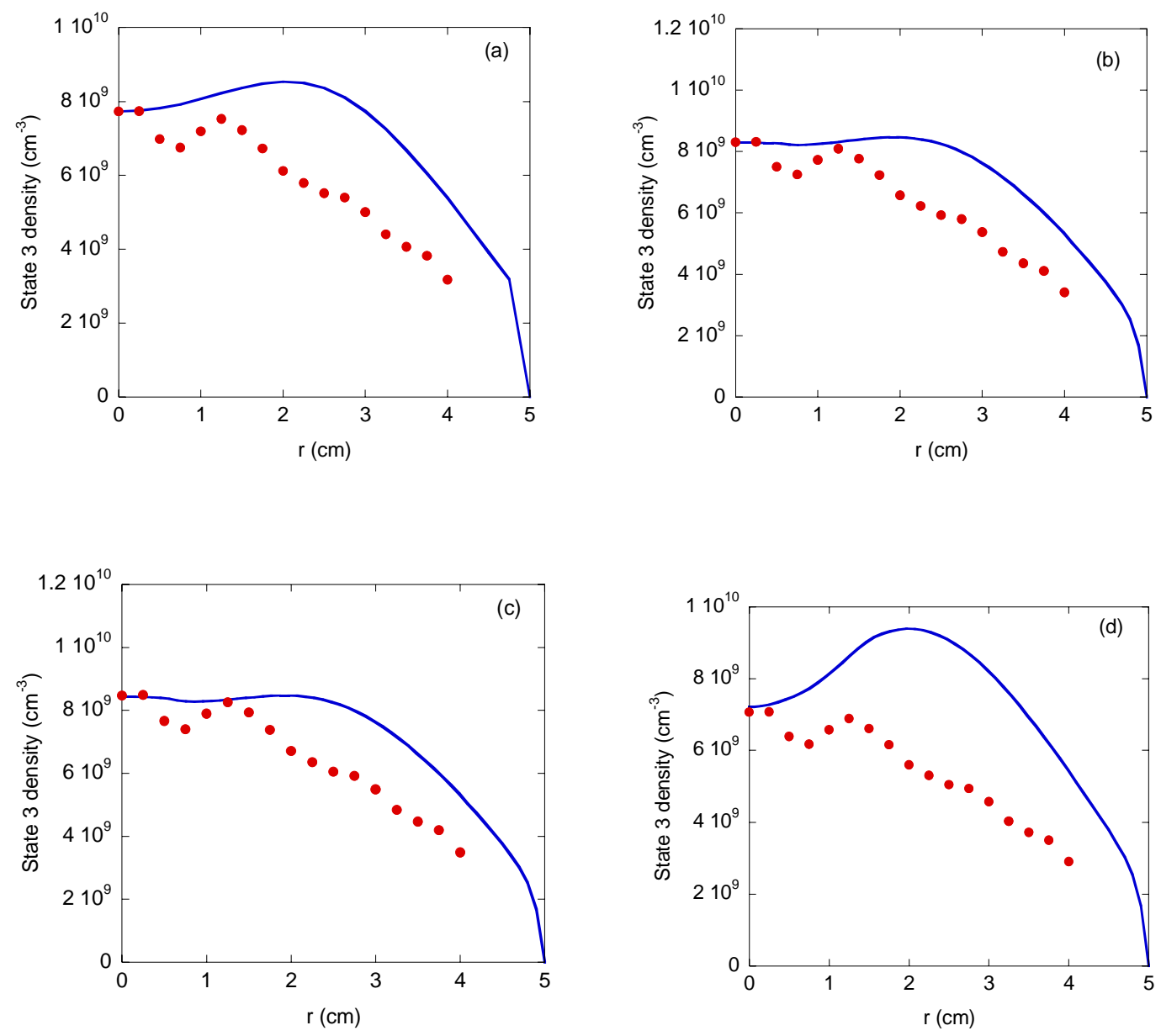

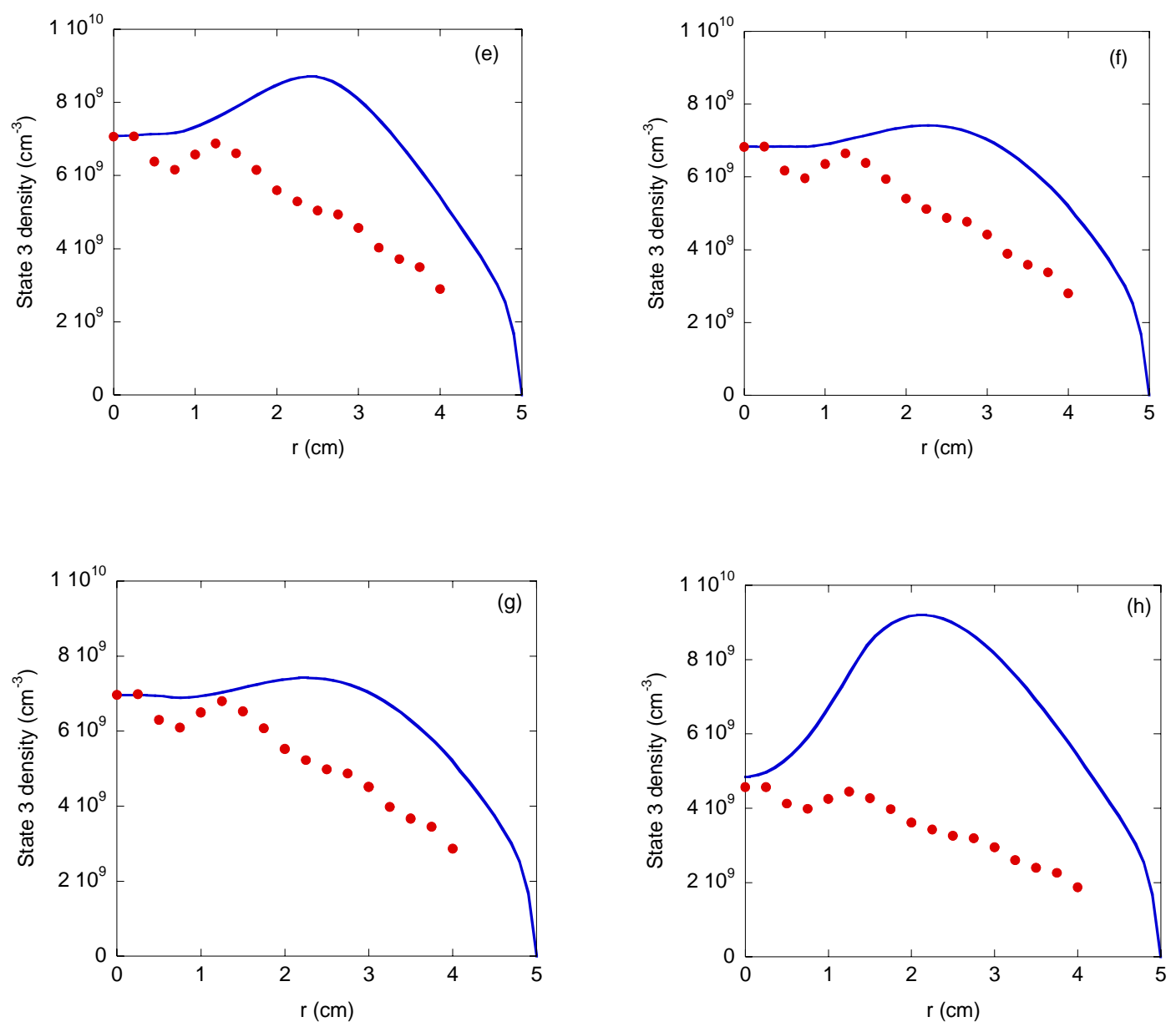

Figure 7.10 Neutral argon State 3 density versus radial position, comparison between experimental data (circles) and CR model output (solid line) with model parameters of a) $P=0.1, W=8.0$, and $12 \mathrm{eV}$ electron beam, b) $P=0.1, W=8.0$, and $20 \mathrm{eV}$ electron beam, c) $P=0.1, W=8.0$, and $40 \mathrm{eV}$ electron beam, d) $P=$ $0.2, W=3.0$, and $20 \mathrm{eV}$ electron beam, e) $P=0.2, W=5.0$, and $40 \mathrm{eV}$ electron beam, f) $P=0.2, W=8.0$, and $20 \mathrm{eV}$ electron beam, g) $P=0.2, W=8.0$, and $40 \mathrm{eV}$ electron beam, and h) $P=0.4, W=3.0$, and $20 \mathrm{eV}$ electron beam. The LIF data are normalized to the model at $r=0$. 

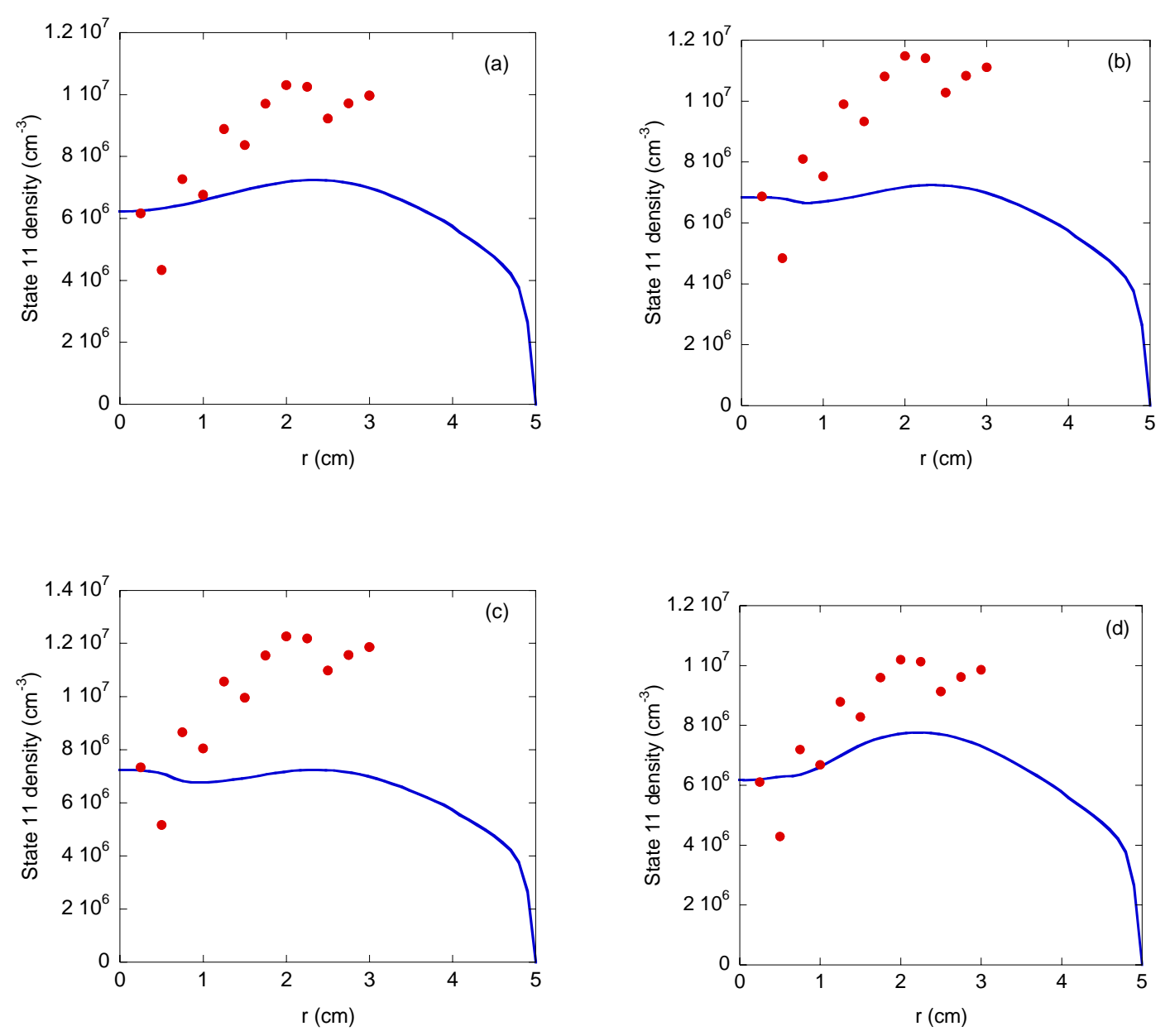

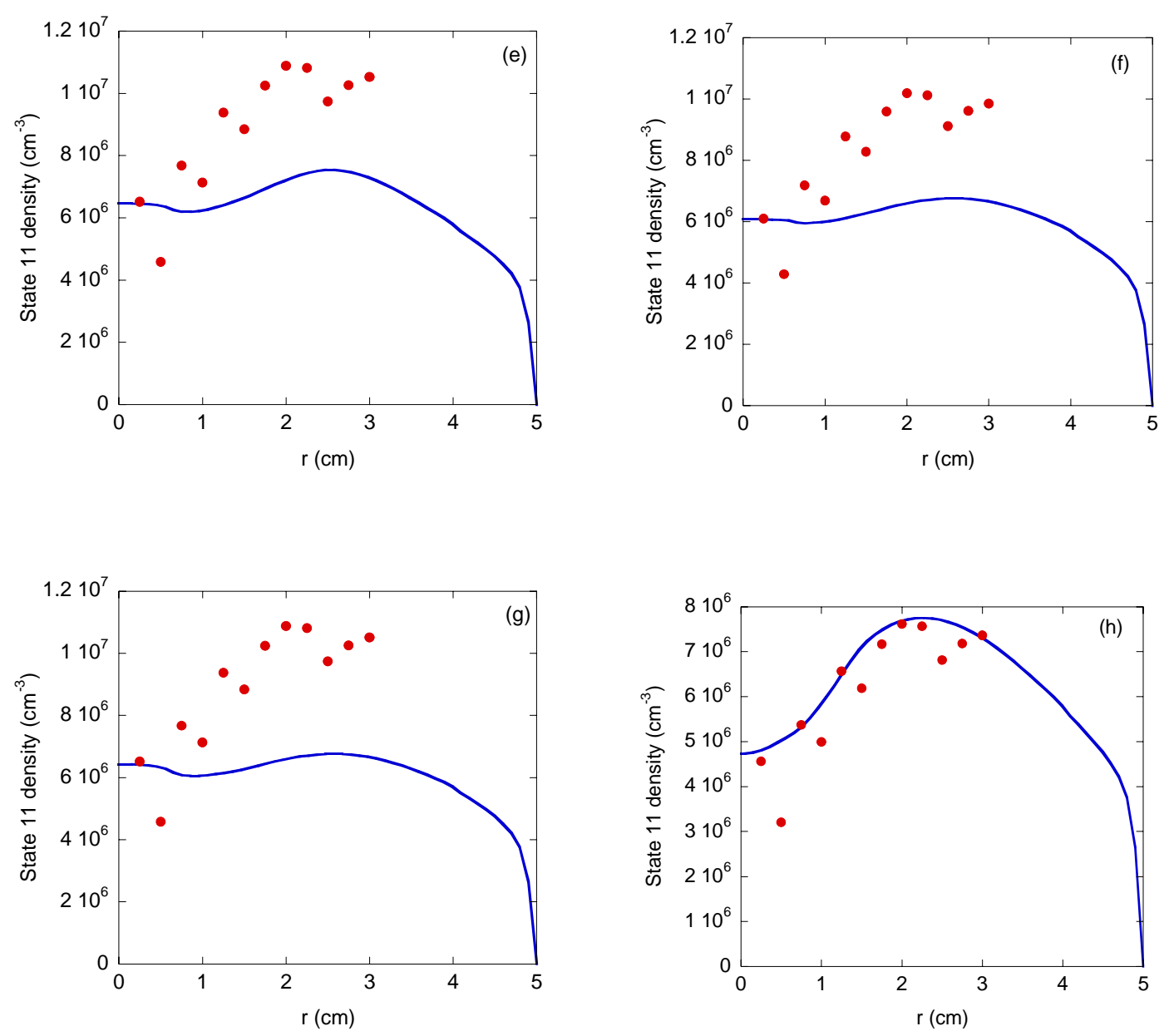

Figure 7.11 Neutral argon State 11 density versus radial position, comparison between experimental data (circles) and CR model output (solid line) with model parameters of a) $P=0.1, W=8.0$, and $12 \mathrm{eV}$ electron beam, b) $P=0.1, W=8.0$, and $20 \mathrm{eV}$ electron beam, c) $P=0.1, W=8.0$, and $40 \mathrm{eV}$ electron beam, d) $P=$ $0.2, W=3.0$, and $20 \mathrm{eV}$ electron beam, e) $P=0.2, W=5.0$, and $40 \mathrm{eV}$ electron beam, f) $P=0.2, W=8.0$, and $20 \mathrm{eV}$ electron beam, g) $P=0.2, W=8.0$, and $40 \mathrm{eV}$ electron beam, and h) $P=0.4, W=3.0$, and $20 \mathrm{eV}$ electron beam. The data are normalized to the model at $r=0$.

The next CR model considered combined an off-axis ground state depletion with an off-axis electron beam. The comparisons for the best match of both State 3 and State 11 between the CR model prediction and the experimental data are shown in Figure 7.12. This was for a neutral ground state with $P=0.2, W=1.0$, and $C=2.5$ and a $40 \mathrm{eV}$ electron beam located at $1.0 \mathrm{~cm}<r<2.0 \mathrm{~cm}$. It can be seen that matching both states 
would require a decrease in the $\mathrm{CR}$ model predictions for State 3 density and a sharp increase in the State 11 density for $1.0 \mathrm{~cm}<r<3.0 \mathrm{~cm}$.
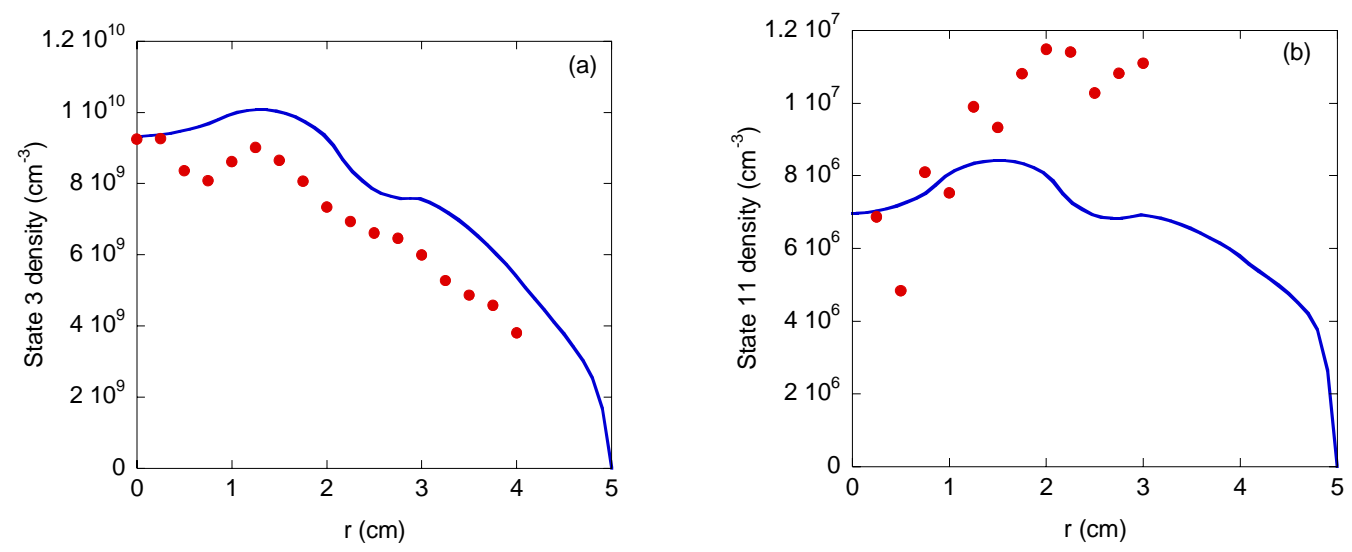

Figure 7.12 Neutral argon excited density versus radial position, comparison between experimental data (circles) and CR model output (solid line) with model parameters of $P=0.2, W=1.0$, and $C=2.5$ and 40 eV electron beam at $1.0<r<2.0$ for a) State 3 and b) State 11 .

Finally, we combined an on-axis ground state depletion with an off-axis electron beam. The comparisons of both State 3 and State 11 between the CR model prediction for a neutral ground state with $P=0.2, W=3.0$, and a $20 \mathrm{eV}$ electron beam located at $2.0 \mathrm{~cm}$ $<r<3.0 \mathrm{~cm}$ and the experimental data are shown in Figure 7.13. While these parameters give a reasonable match between the CR model prediction and the data for State 11, the electron beam over $2.0 \mathrm{~cm}<r<3.0 \mathrm{~cm}$ yields a relative State 3 density much larger than what was observed experimentally.

None of the depletion plus electron beam CR models examined were able to reproduce the essential features of the LIF and the emission measurements. Therefore, we are unable to draw any conclusions about the likely neutral density profile or existence of an energetic electron beam in an capacitively coupled helicon plasma. 

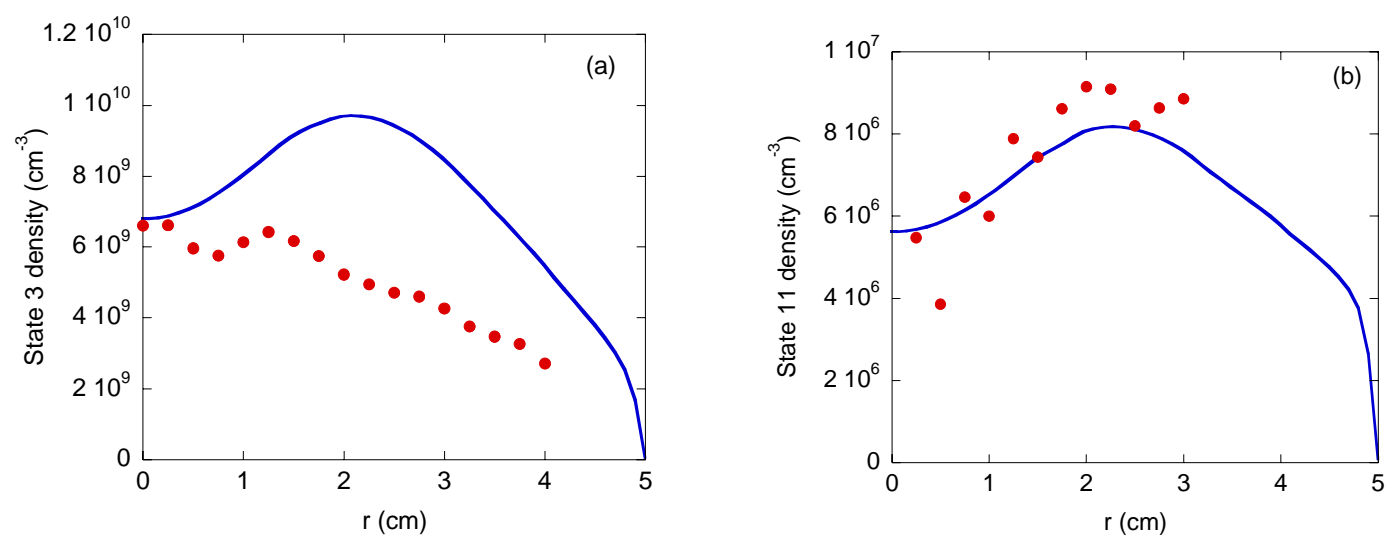

Figure 7.13 Neutral argon excited density versus radial position, comparison between experimental data (circles) and CR model output (solid line) with model parameters of $P=0.2, W=3.0$, and $20 \mathrm{eV}$ electron beam at $2.0<r<3.0$ for a) State 3 and b) State 11 .

\subsection{Parameter Set C (5 mTorr Helicon mode)}

Without a full State 3 density profile from LIF measurements comparison of the CR model and experimental measurements for this source configuration was problematic. If the LIF profile (Figure 6.10) is normalized to that of the $6 \mathrm{mTorr}$ helicon mode case, it can be seen (Figure 7.14) that the increase from the edge to $r=2$ is about the same in both cases. In Figure 7.15, a similar comparison of the Abel inverted spectroscopy data is shown. The inverted emission profiles are more sharply peaked on-axis for the $6 \mathrm{mTorr}$ helicon mode case; likely a result of the larger peak in plasma density for the 6 mTorr case (compare Figure 6.1 to Figure 6.9). This crude profile comparison, along with the experience gained from many CR model runs, suggests a smaller, on-axis neutral depletion for this 5 mTorr helicon source configuration. 


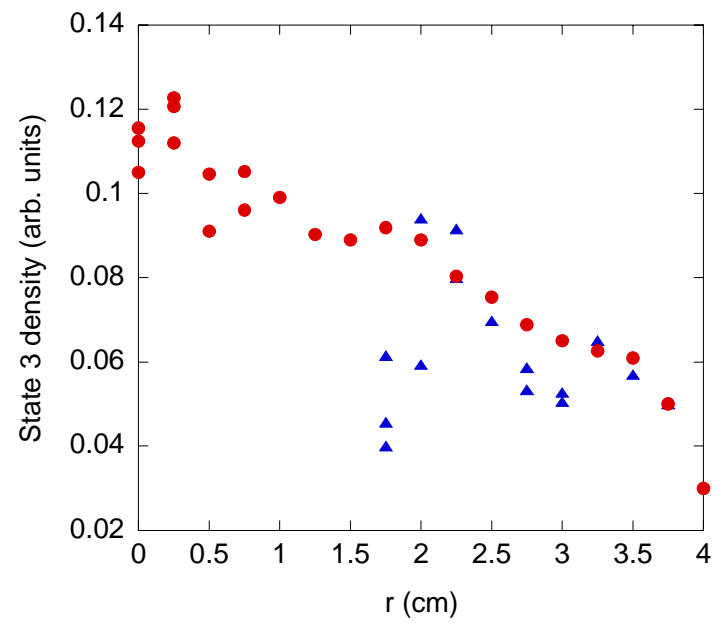

Figure 7.14 Comparison of State 3 density profiles for Parameter Sets A (circles) and B (triangles).
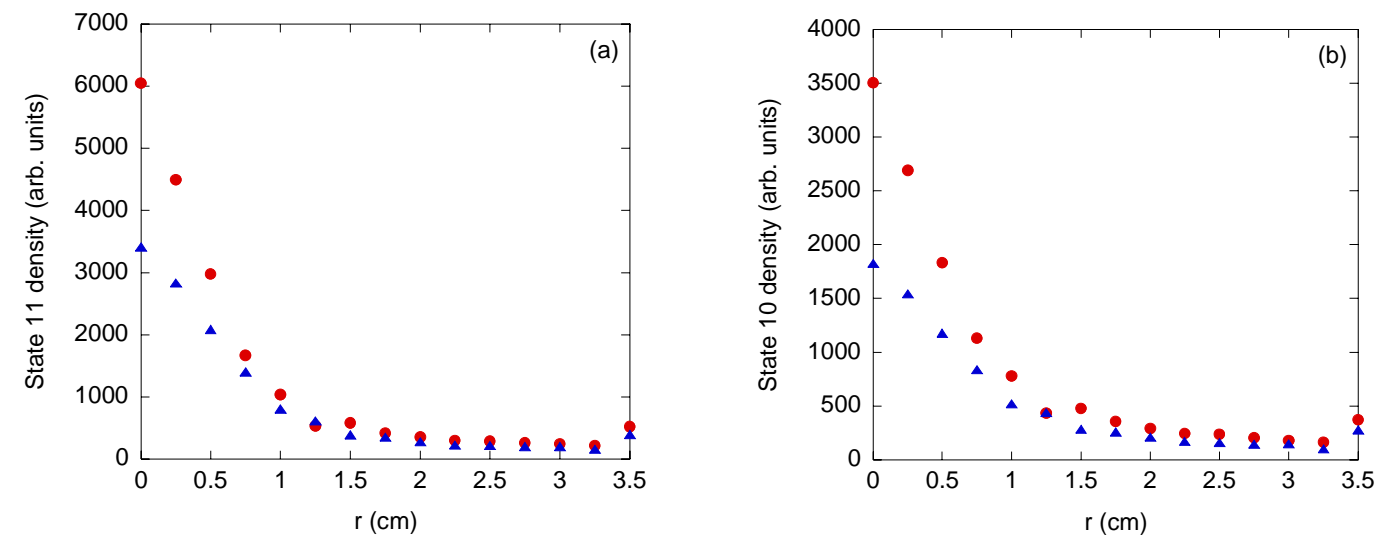

Figure 7.15 Comparison of a) State 11 and b) State 10 density profiles for Parameter Sets A (circles) and B (triangles).

Detailed comparison of CR model predictions to the inverted spectroscopy measurements yielded a best match for a ground state depletion of $20 \%$ with a width of 3 $\mathrm{cm}(P=0.2, W=3)$. The comparisons are shown in Figure 7.16, and the chi-square test yielded a) 0.13 and b) 0.12 . Therefore, consistent with the crude profile analysis, comparison with the CR model indicates that the $5 \mathrm{mT}$ Torr helicon plasma has a smaller, 
$\sim 20 \%$, on-axis neutral depletion. This ground state profile is shown in Figure 7.17. The on-axis ionization fraction for this case is $9.8 \%$, quite a bit lower than for the 6 mTorr helicon case. Again, knowing the depletion yields a more accurate calculation of the ionization fraction because use of the edge neutral pressure would yield an incorrect ionization fraction calculation of $7.8 \%$.
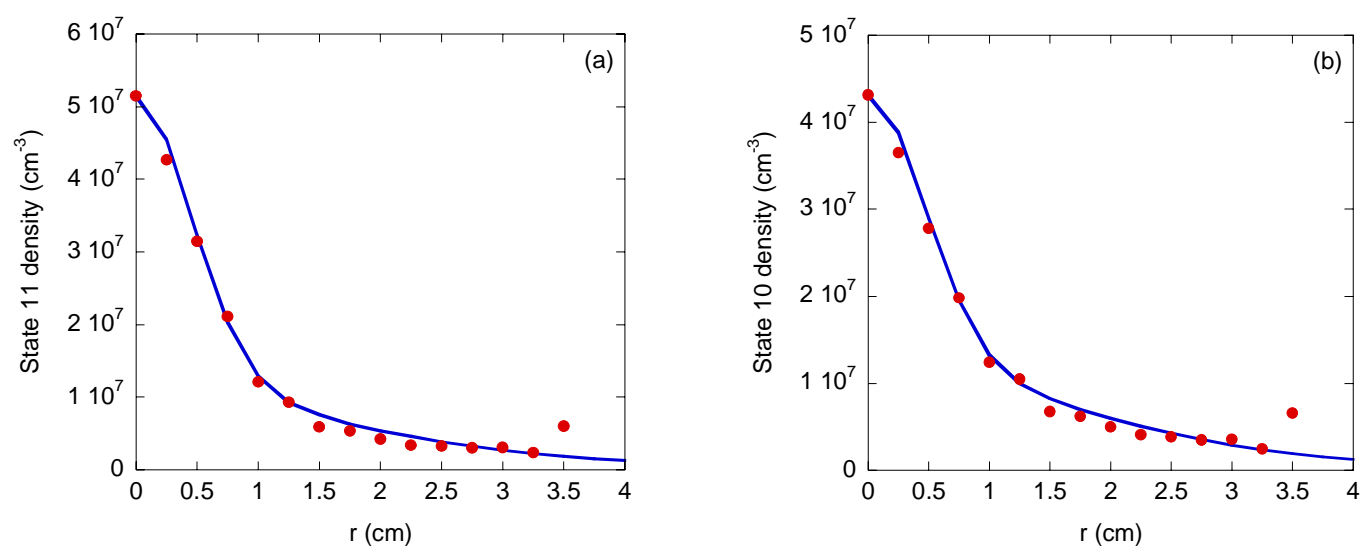

Figure 7.16 Neutral argon excited state density versus radial position, comparison between experimental data (circles) and CR model output (triangles) for a) State 11 and b) State 10.

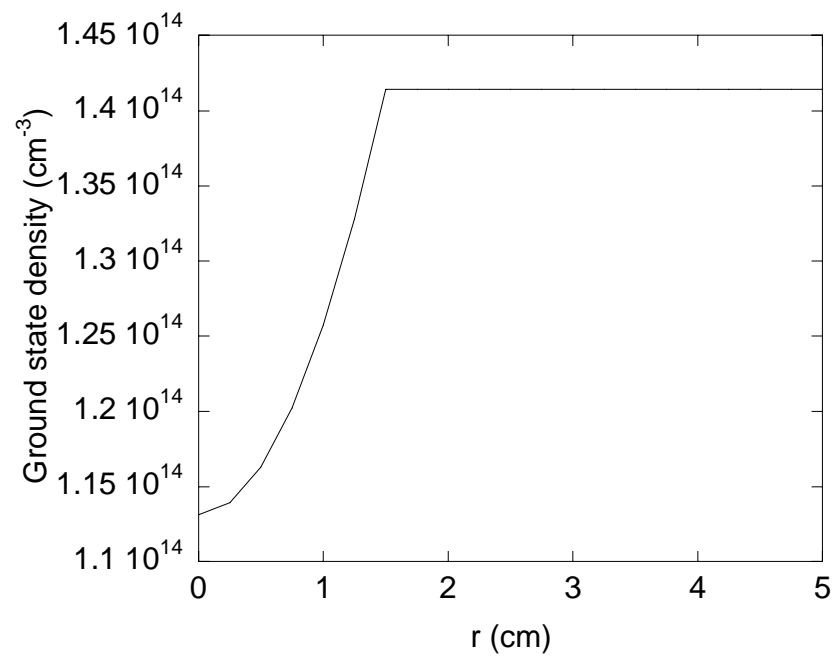

Figure 7.17 Ground state density profile for $P=0.2$ and $W=3 \mathrm{~cm}$. 


\section{References}

${ }^{1}$ F.A. Haas, A. Goodyear, and N.St.J. Braithwaite, Plasma Sources Sci. Technol. 11, 544 (2002). 


\section{Chapter 8: Summary}

We have developed a diode laser-based LIF diagnostic and have demonstrated that it is capable of measuring multiple species in a diverse set of conditions. With a single, low power, tunable diode laser we are able to measure temperatures, densities, and flow velocities of argon atoms, helium atoms, and argon ions. The neutral argon LIF sequence used for this work is a new LIF sequence relying on a nearby metastable state to populate the initial state of the three-level LIF sequence. The diagnostic yields acceptable signal levels for a variety of plasma configurations, including plasma densities as low as $10^{9} \mathrm{~cm}^{-3}$ and in pulsed plasmas. The diagnostic is affordable, portable, and easy to use, making this diagnostic accessible to a wide segment of the plasma research community.

The portability of the diagnostic was demonstrated in a series of experiments at the ANU where we were able to successfully measure flows of argon ions in a low density plasma. Those experiments also demonstrated the efficacy of the heated iodine cell wavelength reference which enabled laser tuning and accurate flow velocity measurements when wavemeters had proven to be unreliable. Even though wavemeters have been generally used to determine the relative spacing of the iodine lines with respect to the LIF absorption line, there is some question to the accuracy of absolute wavelengths identified for the iodine lines with a wavemeter. Thus, the use of the iodine fluorescence measurements is necessary to for absolute wavelength references for LIF measurements. Continued improvement in the determination of the absolute iodine line wavelengths would be useful to the LIF community. 
At ANU, we were able to confirm that measurements of an ion beam using LIF correspond to RFEA measurements of an energetic ion population accelerated through the potential gradient of a double layer. This result allows us to continue to compare double layer experimental results from the three different experiments on which we have performed LIF measurements. For the ANU experiment, the LIF measurements of the ion acceleration through the double layer are in good agreement with computer simulations of the phenomenon.

By combining the LIF diagnostic with passive emission spectroscopy and a collisional-radiative model, we have demonstrated a new method for determining ground state density profiles of neutral argon atoms in helicon plasma sources. With the use of CR models for helium atoms ${ }^{1}$ and argon ions ${ }^{2}$, this approach could be extended to the other species measurable with the LIF diagnostic. The combination of spectroscopic measurements and CR model provides a powerful tool for non-perturbatively measuring important parameters of ground state species in low temperature plasmas.

In the process of performing these spectroscopic measurements, we have also determined that the edge neutral pressure varies significantly over the length of the helicon source chamber. The initiation of plasma discharge decreases the pressure at the edge of the main plasma column, while the pressure further upstream increases. Because the pressure is a very important parameter used for determining characteristics of the neutral atoms in the plasma, great care must be taken when using pressure gauges at various axial locations for measurements of the edge neutral density.

We have successfully measured the ground state argon neutral density profile with a depletion accuracy of $\pm 5 \%$. For our 6 mTorr helicon mode case, we found that a 
ground state profile with a $3 \mathrm{~cm}$-wide depletion of $60 \%$ and a purely Maxwellian EEDF (no electron beam) used in the CR model reproduces the excited state profiles measured using the optical diagnostics (both LIF and emission spectroscopy). Additionally, for a 5 mTorr mode case, we found that a smaller neutral ground state depletion of $20 \%$ will reproduce the experimental data, though only emission spectroscopy data were available for this parameter set. A very important result of this analysis was that no electron beam was required to reproduce the helicon mode profiles.

Calculation of the ionization fraction for the two helicon mode cases indicates that the two source configurations, while employing nearly identical source parameters, yield quite different plasmas. Calculations based on the edge neutral pressure yield ionization fractions of $7.8 \%$ and $11 \%$ for the $5 \mathrm{mTorr}$ and $6 \mathrm{mTorr}$ helicon mode cases, respectively. However, calculations using the results from the CR modeling yield ionization fractions of $9.8 \%$ and $28 \%$, respectively. Apparently, only a slight change in plasma parameters leads to a significant difference in RF power coupling and ionization efficiency in the helicon source. For wave propagation studies, such as Alfvén wave propagation studies recently performed in similar helicon sources, ${ }^{3}$ the difference in ionization fraction results in substantially different expectations for wave damping and Alfvén wavelengths. Because the Alfvén wavelength depends on both neutral and plasma densities, accurate knowledge of the neutral density leads to better calculation of the plasma density and better understanding of the propagation and attenuation of Alfvén waves in helicon sources. Therefore, direct measurements of the neutral density on-axis are required to properly interpret many basic plasma physics experiments performed in helicon source plasmas. 
While we were able to identify ground state neutral profiles and EEDFs that reproduced the experimental measurements with the $\mathrm{CR}$ model for the helicon mode plasma parameters, we were not able to do so for the capacitive mode case. We tried ground state depletions both on- and off-axis as well as the presence of an electron beam, but no combination of parameters that we tried was able to simultaneously reproduce the peaked State 3 profile measured using LIF and the States 10 and 11 profiles measured using emission spectroscopy.

The low power of the diode laser did limit the applicability of the diagnostic in some cases. We were generally unable to achieve reasonable signal to noise for measurements of both argon atoms and argon ions at the same set of plasma parameters. This limited our ability to directly study the interactions between the ions and neutrals. It would have been interesting to perform these measurements with our previous diode that could achieve up to $20 \mathrm{~mW}$. Hopefully, diode laser technology will continue to improve and such experiments can be performed in the next few years. 


\section{References}

${ }^{1}$ T. Fujimoto, J. Quant. Spect. Rad. Transfer 21, 439 (1979).

${ }^{2}$ B.F.M Pots, B. van der Sijde, and D.C, Schram, Physica B and C 94, 369 (1978).

${ }^{3}$ C. Watts and J. Hanna, Phys. Plasmas 11, 1358 (2004). 


\section{Appendix A: Pressure Calibration Data}

The pressure gauge calibration for argon and helium with the Baratron at $z=220 \mathrm{~cm}$ was performed in July 2003 by Compton. The pressure readings for argon at $z=80 \mathrm{~cm}$ were performed by Biloiu in February 2005. The gauge calibrations for helium are shown in Figure A.1 and Figure A.2.
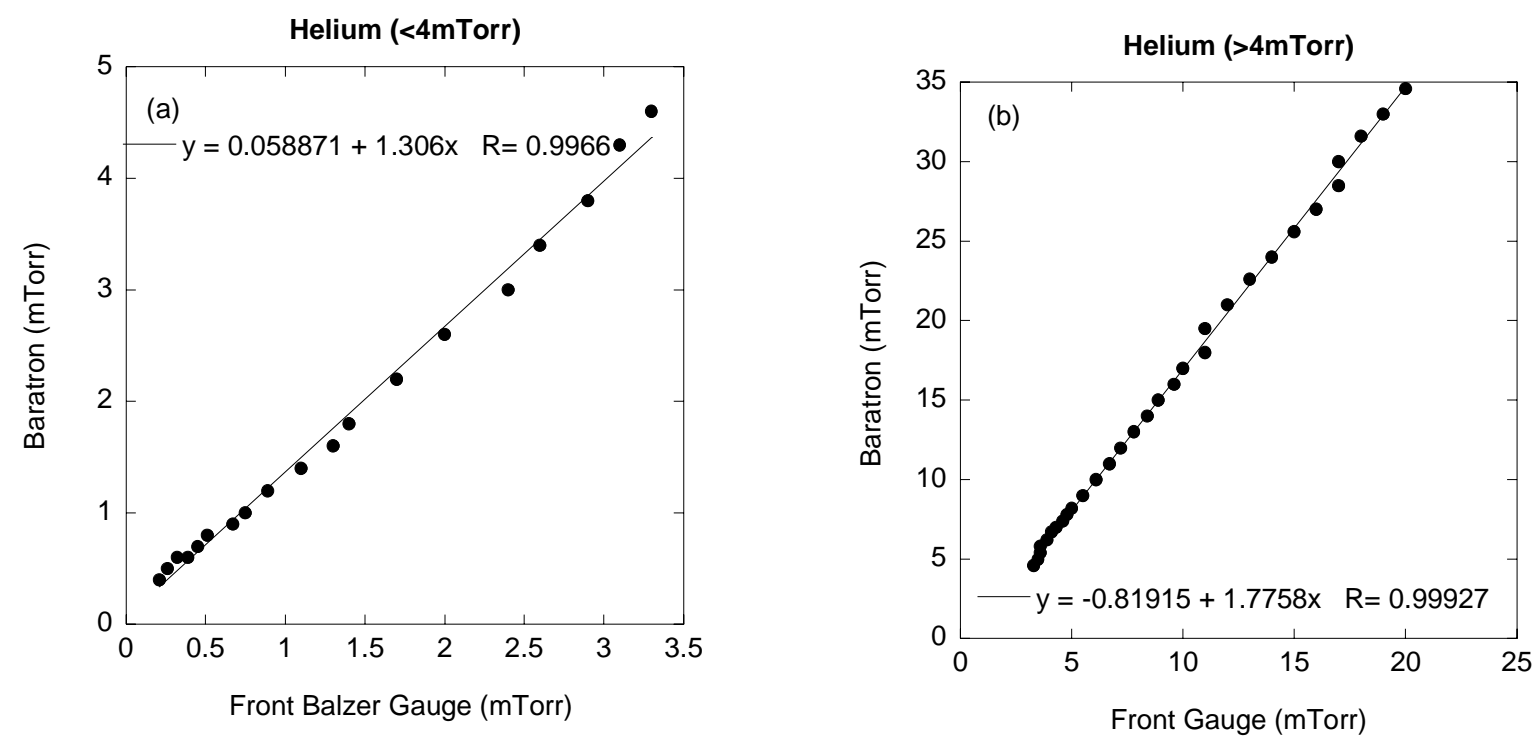

Figure A.1 Pressure gauge calibration for front (HELIX) Balzers gauge using Baratron gauge for helium pressures a) $<4$ mTorr and b) $>4$ mTorr. The linear fit shown is used to convert the gauge pressure reading to real pressure. 

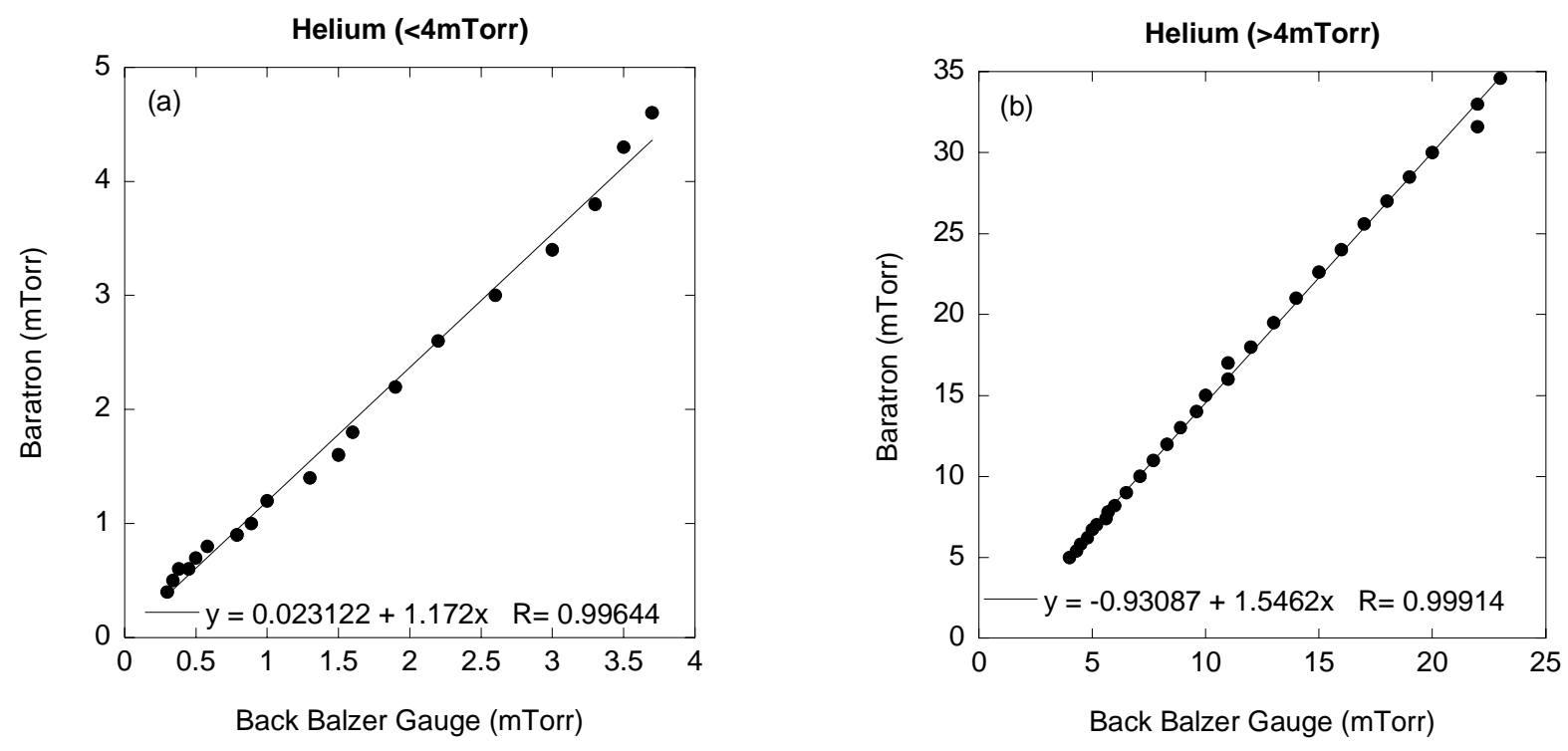

Figure A.2 Pressure gauge calibration for rear (LEIA) Balzers gauge using Baratron gauge for helium pressures a) $<4$ mTorr and $b)>4$ mTorr. The linear fit shown is used to convert the gauge pressure reading to real pressure.

The pressure at front, back, and $\mathrm{z}=220 \mathrm{~cm}$ as a function of gas flow with pressure gradient is shown in Figures.
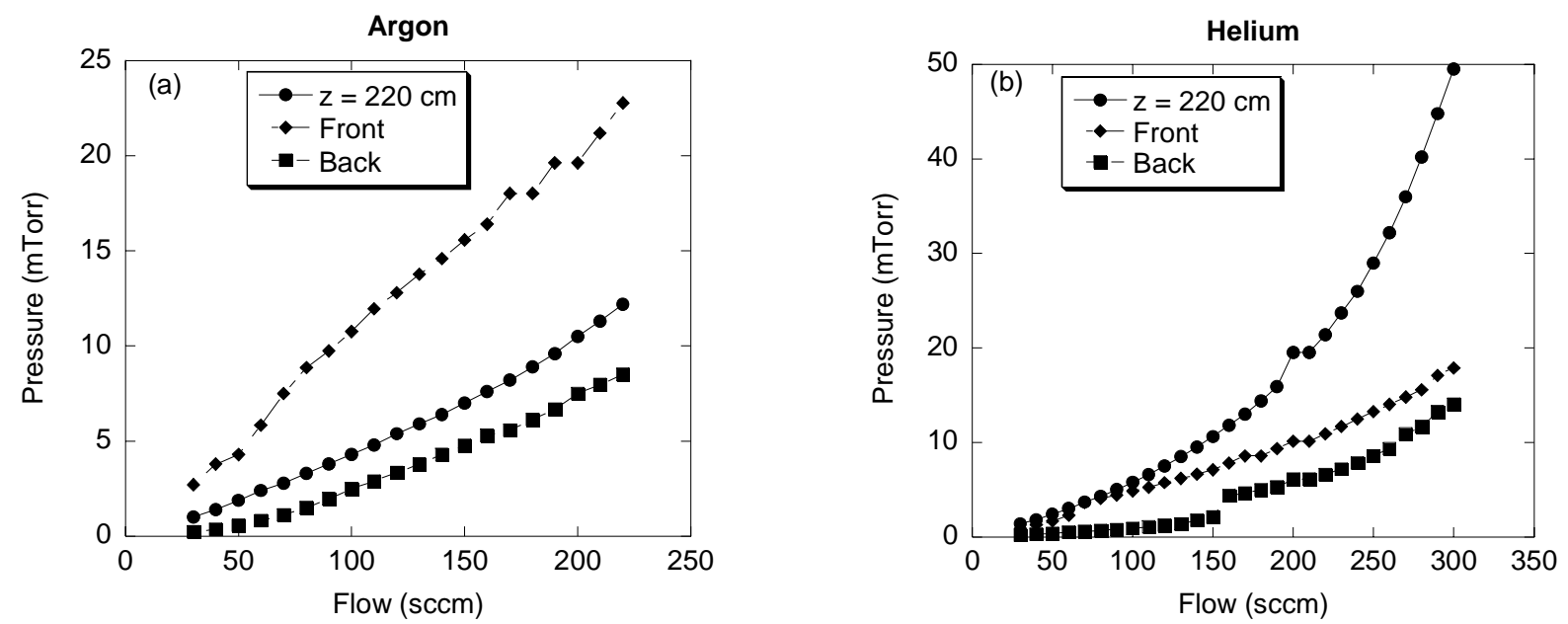

Figure A.3 Real pressure at front and back Balzers gauges and at $\mathrm{z}=220 \mathrm{~cm}$ as a function of a) argon and b) helium gas flow for typical pumping operation.

The data taken by Biloiu for argon with the Baratron located at $z=80 \mathrm{~cm}$ are given in Table A.1.

It is assumed that Equation 2.1 was obtained by a fit of these data, though I was unable to reproduce the 
fit. The columns, from left to right, are interpreted to be readF: the front Balzers gauge reading, readB: the back Balzers gauge reading, baratron: the Baratron gauge reading at $\mathrm{z}=80 \mathrm{~cm}$, realfit: fit to baratron, Chris12: a combination of the following two columns, Chris1: real pressure at the front gauge using Compton's conversion (see Figure 2.4) of readF for P $>4$ mTorr, Chris2: same as Chris1 except for P $<4$ mTorr, Chrisfit: a fit to Chris12, Chrisgr9: (unable to determine), barafit: an alternate fit to baratron, Xuan: real pressure at the front gauge using an older conversion of readF measured by Sun, flow: the argon gas flow in sccm. (All units are in mTorr except where noted.)

Table A.1 Pressure comparison in argon for the Baratron gauge at $\mathrm{z}=80 \mathrm{~cm}$.

\begin{tabular}{|c|c|c|c|c|c|c|c|c|c|c|c|}
\hline readF & readB & baratron & realfit & Chris12 & chris2 & chris1 & chrisfit & Chrisgr9 & barafit & Xuan & Flow \\
\hline 7.8 & & 5.8 & 5.63977 & 12.9 & 12.9 & & 12.89908 & -55.24108 & 5.63961 & 12.78 & 120 \\
\hline 7.2 & & 5.4 & 5.35858 & 11.8 & 11.8 & & 11.81572 & \begin{tabular}{|l|}
-24.40849 \\
\end{tabular} & 5.35849 & 11.77 & 110 \\
\hline 6.6 & 2 & 5 & 4.88789 & 10.7 & 10.7 & & 10.73236 & -7.43994 & 4.88784 & 10.76 & 100 \\
\hline 6 & 1.8 & 4.5 & 4.43393 & 9.6 & 9.6 & & 9.649 & 0.96041 & 4.43391 & 9.72 & 90 \\
\hline 5.5 & 1.6 & 4 & 4.07031 & 8.7 & 8.7 & & 8.7462 & 4.44413 & 4.0703 & 8.85 & 80 \\
\hline 4.8 & 1.3 & 3.5 & 3.51395 & 7.5 & 7.5 & & 7.48228 & 6.11634 & 3.51395 & 7.5 & 70 \\
\hline 4.2 & 1.1 & 3 & 2.95303 & 6.4 & 6.4 & & 6.39892 & 5.62317 & 2.95303 & 5.85 & 60 \\
\hline 3.6 & 0.89 & 2.5 & 2.3342 & 6.8 & & 6.8 & 4.40291 & 4.44491 & 2.3342 & 4.34 & 50 \\
\hline 3.4 & 0.65 & 2 & 2.1263 & 6.3 & & 6.3 & 4.15055 & 4.07314 & 2.1263 & 3.85 & 40 \\
\hline 2.8 & 0.41 & 1.5 & 1.54326 & 3.4 & & 3.4 & 3.39347 & 3.26226 & 1.54325 & 2.48 & 30 \\
\hline 2.5 & 0.32 & 1.25 & 1.29377 & 3 & & 3 & 3.01493 & 2.98381 & 1.29377 & 1.97 & 25 \\
\hline 2 & 0.28 & 1 & 0.96427 & 2.4 & & 2.4 & 2.38403 & 2.4624 & 0.964275 & 1.24 & 20 \\
\hline 1.5 & 0.22 & 0.8 & 0.74081 & 1.8 & & 1.8 & 1.75313 & 1.71712 & 0.740814 & 0.73 & 15 \\
\hline 0.92 & 0.18 & 0.5 & 0.54847 & 1 & & 1 & 1.02129 & 1.02698 & 0.548466 & 0.35 & 10 \\
\hline 0.25 & 0.052 & 0.2 & 0.1909 & 0.2 & & 0.2 & 0.17588 & 0.17561 & 0.190895 & 0.13 & 5 \\
\hline
\end{tabular}




\section{Appendix B: Iodine Cell Fluorescence Data}

Table B.1 Raw data for Figure 3.16(a), iodine fluorescence about Ar II line.

\begin{tabular}{|c|c|c|c|c|}
\hline Wavelength (nm) & $\begin{array}{l}\text { Freqency } \\
(\mathrm{GHz})\end{array}$ & $\begin{array}{l}\text { Iodine } \\
\text { fluorescence } \\
\text { signal } \\
\text { (arbitrary } \\
\text { units) }\end{array}$ & $\begin{array}{l}\text { Frequency } \\
\operatorname{shift}\left(v-v_{0}\right) \\
(\mathrm{GHz})\end{array}$ & $\begin{array}{l}\text { Detrended } \\
\text { iodine } \\
\text { fluorescence } \\
\text { signal (arb. } \\
\text { units) }\end{array}$ \\
\hline 668.6219 & $4.4836 \mathrm{e}+05$ & 0.11475 & -5.6329 & 0.0031330 \\
\hline 668.6219 & $4.4836 \mathrm{e}+05$ & 0.11719 & -5.5666 & 0.0062707 \\
\hline 668.6218 & $4.4836 e+05$ & 0.11963 & -5.5003 & 0.0094074 \\
\hline 668.6212 & $4.4836 \mathrm{e}+05$ & 0.12207 & -5.4340 & 0.012544 \\
\hline 668.6212 & $4.4836 \mathrm{e}+05$ & 0.12695 & -5.3677 & 0.018123 \\
\hline 668.6213 & $4.4836 e+05$ & 0.11475 & -5.3014 & 0.0066114 \\
\hline 668.6211 & $4.4836 e+05$ & 0.11719 & -5.2351 & 0.0097491 \\
\hline 668.6218 & $4.4836 \mathrm{e}+05$ & 0.10742 & $\begin{array}{l}-5.1688 \\
\end{array}$ & 0.00067878 \\
\hline 668.6218 & $4.4836 e+05$ & 0.11231 & -5.1025 & 0.0062575 \\
\hline 668.6218 & $4.4836 \mathrm{e}+05$ & 0.11963 & -5.0362 & 0.014277 \\
\hline 668.6206 & $4.4836 \mathrm{e}+05$ & 0.10986 & -4.9699 & 0.0052068 \\
\hline 668.6205 & $4.4836 \mathrm{e}+05$ & 0.12695 & -4.9036 & 0.022993 \\
\hline 668.6206 & $4.4836 \mathrm{e}+05$ & 0.13916 & -4.8373 & 0.035895 \\
\hline 668.6211 & $4.4836 \mathrm{e}+05$ & 0.13428 & -4.7710 & 0.031708 \\
\hline 668.6210 & $4.4836 \mathrm{e}+05$ & 0.14893 & -4.7047 & 0.047053 \\
\hline 668.6210 & $4.4836 \mathrm{e}+05$ & 0.18311 & -4.6384 & 0.081927 \\
\hline 668.6202 & $4.4836 \mathrm{e}+05$ & 0.21240 & $\begin{array}{l}-4.5721 \\
\end{array}$ & 0.11192 \\
\hline 668.6201 & $4.4836 \mathrm{e}+05$ & 0.25146 & -4.5058 & 0.15168 \\
\hline 668.6201 & $4.4836 \mathrm{e}+05$ & 0.25635 & -4.4395 & 0.15726 \\
\hline 668.6201 & $4.4836 \mathrm{e}+05$ & 0.26855 & -4.3732 & 0.17016 \\
\hline 668.6206 & $4.4836 \mathrm{e}+05$ & 0.31738 & $\begin{array}{l}-4.3069 \\
\end{array}$ & 0.21968 \\
\hline 668.6205 & $4.4836 \mathrm{e}+05$ & 0.33935 & -4.2406 & 0.24235 \\
\hline 668.6206 & $4.4836 \mathrm{e}+05$ & 0.39062 & -4.1743 & 0.29432 \\
\hline 668.6195 & $4.4836 \mathrm{e}+05$ & 0.42480 & -4.1080 & 0.32919 \\
\hline 668.6196 & $4.4836 \mathrm{e}+05$ & 0.43945 & -4.0417 & 0.34454 \\
\hline 668.6197 & $4.4836 \mathrm{e}+05$ & 0.49072 & -3.9754 & 0.39650 \\
\hline 668.6204 & $4.4836 \mathrm{e}+05$ & 0.54687 & -3.9091 & 0.45335 \\
\hline 668.6203 & $4.4836 \mathrm{e}+05$ & 0.59082 & -3.8428 & 0.49799 \\
\hline 668.6204 & $4.4836 \mathrm{e}+05$ & 0.60547 & -3.7765 & 0.51334 \\
\hline 668.6196 & $4.4836 \mathrm{e}+05$ & 0.60303 & -3.7102 & 0.51159 \\
\hline 668.6196 & $4.4836 \mathrm{e}+05$ & 0.62012 & -3.6439 & 0.52937 \\
\hline 668.6195 & $4.4836 \mathrm{e}+05$ & 0.62988 & -3.5776 & 0.53984 \\
\hline 668.6196 & $4.4836 \mathrm{e}+05$ & 0.63232 & -3.5113 & 0.54297 \\
\hline 668.6198 & $4.4836 \mathrm{e}+05$ & 0.61523 & -3.4450 & 0.52658 \\
\hline 668.6197 & $4.4836 \mathrm{e}+05$ & 0.61768 & -3.3787 & 0.52972 \\
\hline 668.6190 & $4.4836 \mathrm{e}+05$ & 0.59815 & -3.3124 & 0.51088 \\
\hline 668.6191 & $4.4836 \mathrm{e}+05$ & 0.56885 & -3.2461 & 0.48228 \\
\hline 668.6190 & $4.4836 \mathrm{e}+05$ & 0.50537 & -3.1798 & 0.41950 \\
\hline 668.6190 & $4.4836 e+05$ & 0.43457 & -3.1135 & 0.34939 \\
\hline 668.6195 & $4.4836 \mathrm{e}+05$ & 0.41260 & -3.0472 & 0.32812 \\
\hline 668.6195 & $4.4836 \mathrm{e}+05$ & 0.34912 & -2.9809 & 0.26534 \\
\hline 668.6194 & $4.4836 e+05$ & 0.29541 & -2.9146 & 0.21232 \\
\hline 668.6184 & $4.4836 \mathrm{e}+05$ & 0.24414 & -2.8483 & 0.16175 \\
\hline 668.6185 & $4.4836 \mathrm{e}+05$ & 0.20996 & -2.7820 & 0.12826 \\
\hline
\end{tabular}




\begin{tabular}{|c|c|c|c|c|}
\hline 668.6184 & $4.4836 \mathrm{e}+05$ & 0.18066 & -2.7157 & 0.099661 \\
\hline 668.6188 & $4.4836 \mathrm{e}+05$ & 0.18311 & -2.6494 & 0.10280 \\
\hline 668.6187 & $4.4836 \mathrm{e}+05$ & 0.16602 & -2.5831 & 0.086405 \\
\hline 668.6187 & $4.4836 \mathrm{e}+05$ & 0.13672 & -2.5168 & 0.057803 \\
\hline 668.6179 & $4.4836 \mathrm{e}+05$ & 0.12451 & -2.4505 & 0.046292 \\
\hline 668.6179 & $4.4836 \mathrm{e}+05$ & 0.11475 & -2.3842 & 0.037222 \\
\hline 668.6183 & $4.4836 \mathrm{e}+05$ & 0.11231 & -2.3179 & 0.035476 \\
\hline 668.6183 & $4.4836 \mathrm{e}+05$ & 0.10498 & -2.2516 & 0.028847 \\
\hline 668.6183 & $4.4836 \mathrm{e}+05$ & 0.10010 & -2.1853 & 0.024661 \\
\hline 668.6182 & $4.4836 \mathrm{e}+05$ & 0.090332 & -2.1190 & 0.015590 \\
\hline 668.6175 & $4.4836 \mathrm{e}+05$ & 0.078125 & -2.0527 & 0.0040790 \\
\hline 668.6174 & $4.4836 \mathrm{e}+05$ & 0.078125 & -1.9864 & 0.0047747 \\
\hline 668.6174 & $4.4836 \mathrm{e}+05$ & 0.080566 & -1.9201 & 0.0079114 \\
\hline 668.6174 & $4.4836 \mathrm{e}+05$ & 0.075684 & -1.8538 & 0.0037251 \\
\hline 668.6177 & $4.4836 \mathrm{e}+05$ & 0.068359 & -1.7875 & -0.0029042 \\
\hline 668.6176 & $4.4836 \mathrm{e}+05$ & 0.051270 & -1.7212 & -0.019298 \\
\hline 668.6178 & $4.4836 \mathrm{e}+05$ & 0.048828 & -1.6549 & -0.021044 \\
\hline 668.6167 & $4.4836 \mathrm{e}+05$ & 0.036621 & -1.5886 & -0.032555 \\
\hline 668.6165 & $4.4836 \mathrm{e}+05$ & 0.036621 & -1.5223 & -0.031859 \\
\hline 668.6167 & $4.4836 \mathrm{e}+05$ & 0.026855 & -1.4560 & -0.040930 \\
\hline 668.6171 & $4.4836 \mathrm{e}+05$ & 0.024414 & -1.3897 & -0.042675 \\
\hline 668.6171 & $4.4836 \mathrm{e}+05$ & 0.024414 & -1.3234 & -0.041979 \\
\hline 668.6169 & $4.4836 \mathrm{e}+05$ & 0.034180 & -1.2571 & -0.031518 \\
\hline 668.6164 & $4.4836 \mathrm{e}+05$ & 0.053711 & -1.1908 & -0.011291 \\
\hline 668.6163 & $4.4836 \mathrm{e}+05$ & 0.051270 & -1.1245 & -0.013036 \\
\hline 668.6165 & $4.4836 \mathrm{e}+05$ & 0.080566 & -1.0582 & 0.016955 \\
\hline 668.6165 & $4.4836 \mathrm{e}+05$ & 0.095215 & -0.99190 & 0.032300 \\
\hline 668.6166 & $4.4836 \mathrm{e}+05$ & 0.097656 & -0.92560 & 0.035437 \\
\hline 668.6166 & $4.4836 \mathrm{e}+05$ & 0.11963 & -0.85930 & 0.058105 \\
\hline 668.6167 & $4.4836 \mathrm{e}+05$ & 0.13428 & -0.79300 & 0.073449 \\
\hline 668.6158 & $4.4836 \mathrm{e}+05$ & 0.14893 & -0.72670 & 0.088794 \\
\hline 668.6158 & $4.4836 \mathrm{e}+05$ & 0.15869 & -0.66040 & 0.099254 \\
\hline 668.6158 & $4.4836 \mathrm{e}+05$ & 0.16846 & -0.59410 & 0.10972 \\
\hline 668.6162 & $4.4836 \mathrm{e}+05$ & 0.17578 & -0.52780 & 0.11774 \\
\hline 668.6162 & $4.4836 \mathrm{e}+05$ & 0.20019 & -0.46150 & 0.14285 \\
\hline 668.6161 & $4.4836 \mathrm{e}+05$ & 0.23193 & -0.39520 & 0.17528 \\
\hline 668.6150 & $4.4836 \mathrm{e}+05$ & 0.26611 & -0.32890 & 0.21015 \\
\hline 668.6151 & $4.4836 \mathrm{e}+05$ & 0.32471 & -0.26260 & 0.26944 \\
\hline 668.6149 & $4.4836 \mathrm{e}+05$ & 0.31982 & -0.19630 & 0.26526 \\
\hline 668.6155 & $4.4836 \mathrm{e}+05$ & 0.40283 & -0.13000 & 0.34896 \\
\hline 668.6155 & $4.4836 \mathrm{e}+05$ & 0.51270 & -0.063700 & 0.45952 \\
\hline 668.6155 & $4.4836 \mathrm{e}+05$ & 0.65918 & 0.0026000 & 0.60670 \\
\hline 668.6144 & $4.4836 \mathrm{e}+05$ & 0.82031 & 0.068900 & 0.76853 \\
\hline 668.6144 & $4.4836 \mathrm{e}+05$ & 0.83740 & 0.13520 & 0.78631 \\
\hline 668.6143 & $4.4836 \mathrm{e}+05$ & 0.94727 & 0.20150 & 0.89687 \\
\hline 668.6145 & $4.4836 \mathrm{e}+05$ & 1.0107 & 0.26780 & 0.96104 \\
\hline 668.6147 & $4.4836 \mathrm{e}+05$ & 0.99121 & 0.33410 & 0.94221 \\
\hline 668.6147 & $4.4836 \mathrm{e}+05$ & 0.92285 & 0.40040 & 0.87455 \\
\hline 668.6148 & $4.4836 \mathrm{e}+05$ & 0.92529 & 0.46670 & 0.87768 \\
\hline 668.6140 & $4.4836 \mathrm{e}+05$ & 0.84717 & 0.53300 & 0.80025 \\
\hline 668.6139 & $4.4836 \mathrm{e}+05$ & 0.75440 & 0.59930 & 0.70818 \\
\hline 668.6140 & $4.4836 \mathrm{e}+05$ & 0.60791 & 0.66560 & 0.56239 \\
\hline 668.6144 & $4.4836 \mathrm{e}+05$ & 0.43213 & 0.73190 & 0.38730 \\
\hline
\end{tabular}




\begin{tabular}{|c|c|c|c|c|}
\hline 668.6143 & $4.4836 \mathrm{e}+05$ & 0.27588 & 0.79820 & 0.23175 \\
\hline 668.6143 & $4.4836 \mathrm{e}+05$ & 0.26367 & 0.86450 & 0.22024 \\
\hline 668.6133 & $4.4836 \mathrm{e}+05$ & 0.13428 & 0.93080 & 0.091537 \\
\hline 668.6135 & $4.4836 \mathrm{e}+05$ & 0.070801 & 0.99710 & 0.028757 \\
\hline 668.6134 & $4.4836 \mathrm{e}+05$ & 0.029297 & 1.0634 & -0.012052 \\
\hline 668.6133 & $4.4836 \mathrm{e}+05$ & 0.014648 & 1.1297 & -0.026005 \\
\hline 668.6133 & $4.4836 \mathrm{e}+05$ & 0.0048830 & 1.1960 & -0.035074 \\
\hline 668.6134 & $4.4836 \mathrm{e}+05$ & 0.017090 & 1.2623 & -0.022172 \\
\hline 668.6127 & $4.4836 \mathrm{e}+05$ & 0.0097660 & 1.3286 & -0.028800 \\
\hline 668.6128 & $4.4836 \mathrm{e}+05$ & 0.0097660 & 1.3949 & -0.028104 \\
\hline 668.6127 & $4.4836 \mathrm{e}+05$ & 0.012207 & 1.4612 & -0.024968 \\
\hline 668.6127 & $4.4836 \mathrm{e}+05$ & 0.021973 & 1.5275 & -0.014506 \\
\hline 668.6128 & $4.4836 \mathrm{e}+05$ & 0.034180 & 1.5938 & -0.0016033 \\
\hline 668.6127 & $4.4836 \mathrm{e}+05$ & 0.041504 & 1.6601 & 0.0064164 \\
\hline 668.6125 & $4.4836 \mathrm{e}+05$ & 0.058594 & 1.7264 & 0.024202 \\
\hline 668.6117 & $4.4836 \mathrm{e}+05$ & 0.080566 & 1.7927 & 0.046870 \\
\hline 668.6117 & $4.4836 \mathrm{e}+05$ & 0.078125 & 1.8590 & 0.045124 \\
\hline 668.6116 & $4.4836 \mathrm{e}+05$ & 0.10010 & 1.9253 & 0.067793 \\
\hline 668.6121 & $4.4836 \mathrm{e}+05$ & 0.10498 & 1.9916 & 0.073371 \\
\hline 668.6122 & $4.4836 \mathrm{e}+05$ & 0.10742 & 2.0579 & 0.076509 \\
\hline 668.6122 & $4.4836 \mathrm{e}+05$ & 0.11231 & 2.1242 & 0.082087 \\
\hline 668.6111 & $4.4836 \mathrm{e}+05$ & 0.12451 & 2.1905 & 0.094990 \\
\hline 668.6111 & $4.4836 \mathrm{e}+05$ & 0.11231 & 2.2568 & 0.083479 \\
\hline 668.6111 & $4.4836 \mathrm{e}+05$ & 0.11719 & 2.3231 & 0.089057 \\
\hline 668.6112 & $4.4836 \mathrm{e}+05$ & 0.10254 & 2.3894 & 0.075104 \\
\hline 668.6113 & $4.4836 \mathrm{e}+05$ & 0.10986 & 2.4557 & 0.083124 \\
\hline 668.6115 & $4.4836 \mathrm{e}+05$ & 0.092773 & 2.5220 & 0.066729 \\
\hline 668.6115 & $4.4836 e+05$ & 0.10010 & 2.5883 & 0.074750 \\
\hline 668.6104 & $4.4836 \mathrm{e}+05$ & 0.087891 & 2.6546 & 0.063239 \\
\hline 668.6105 & $4.4836 \mathrm{e}+05$ & 0.083008 & 2.7209 & 0.059051 \\
\hline 668.6104 & $4.4836 \mathrm{e}+05$ & 0.080566 & 2.7872 & 0.057305 \\
\hline 668.6108 & $4.4836 \mathrm{e}+05$ & 0.080566 & 2.8535 & 0.058001 \\
\hline 668.6110 & $4.4836 \mathrm{e}+05$ & 0.073242 & 2.9198 & 0.051372 \\
\hline 668.6110 & $4.4836 \mathrm{e}+05$ & 0.043945 & 2.9861 & 0.022771 \\
\hline 668.6098 & $4.4836 \mathrm{e}+05$ & 0.031738 & 3.0524 & 0.011260 \\
\hline 668.6096 & $4.4836 \mathrm{e}+05$ & 0.014648 & 3.1187 & -0.0051345 \\
\hline 668.6097 & $4.4836 \mathrm{e}+05$ & 0.012207 & 3.1850 & -0.0068798 \\
\hline 668.6101 & $4.4836 \mathrm{e}+05$ & 0.0048830 & 3.2513 & -0.013508 \\
\hline 668.6099 & $4.4836 \mathrm{e}+05$ & 0.0000 & 3.3176 & -0.017695 \\
\hline 668.6100 & $4.4836 \mathrm{e}+05$ & 0.0000 & 3.3839 & -0.017000 \\
\hline 668.6088 & $4.4836 \mathrm{e}+05$ & 0.0097660 & 3.4502 & -0.0065381 \\
\hline 668.6088 & $4.4836 \mathrm{e}+05$ & 0.0097660 & 3.5165 & -0.0058424 \\
\hline 668.6089 & $4.4836 \mathrm{e}+05$ & 0.0073240 & 3.5828 & -0.0075887 \\
\hline 668.6088 & $4.4836 \mathrm{e}+05$ & 0.012207 & 3.6491 & -0.0020100 \\
\hline 668.6094 & $4.4836 \mathrm{e}+05$ & 0.017090 & 3.7154 & 0.0035687 \\
\hline 668.6093 & $4.4836 \mathrm{e}+05$ & 0.034180 & 3.7817 & 0.021354 \\
\hline 668.6094 & $4.4836 \mathrm{e}+05$ & 0.051270 & 3.8480 & 0.039140 \\
\hline 668.6082 & $4.4836 \mathrm{e}+05$ & 0.065918 & 3.9143 & 0.054484 \\
\hline 668.6083 & $4.4836 \mathrm{e}+05$ & 0.075684 & 3.9806 & 0.064945 \\
\hline 668.6081 & $4.4836 \mathrm{e}+05$ & 0.083008 & 4.0469 & 0.072965 \\
\hline 668.6084 & $4.4836 \mathrm{e}+05$ & 0.10010 & 4.1132 & 0.090751 \\
\hline 668.6083 & $4.4836 \mathrm{e}+05$ & 0.12695 & 4.1795 & 0.11830 \\
\hline 668.6083 & $4.4836 \mathrm{e}+05$ & 0.14404 & 4.2458 & 0.13609 \\
\hline
\end{tabular}




\begin{tabular}{|l|l|l|l|l|}
\hline 668.6076 & $4.4836 \mathrm{e}+05$ & 0.14648 & 4.3121 & 0.13922 \\
\hline 668.6076 & $4.4836 \mathrm{e}+05$ & 0.15869 & 4.3784 & 0.15213 \\
\hline 668.6075 & $4.4836 \mathrm{e}+05$ & 0.15625 & 4.4447 & 0.15038 \\
\hline 668.6078 & $4.4836 \mathrm{e}+05$ & 0.15381 & 4.5110 & 0.14864 \\
\hline 668.6078 & $4.4836 \mathrm{e}+05$ & 0.14893 & 4.5773 & 0.14445 \\
\hline 668.6077 & $4.4836 \mathrm{e}+05$ & 0.12695 & 4.6436 & 0.12317 \\
\hline 668.6068 & $4.4837 \mathrm{e}+05$ & 0.11475 & 4.7099 & 0.11166 \\
\hline 668.6067 & $4.4837 \mathrm{e}+05$ & 0.080566 & 4.7762 & 0.078176 \\
\hline 668.6066 & $4.4837 \mathrm{e}+05$ & 0.048828 & 4.8425 & 0.047133 \\
\hline 668.6066 & $4.4837 \mathrm{e}+05$ & 0.026855 & 4.9088 & 0.025856 \\
\hline 668.6072 & $4.4836 \mathrm{e}+05$ & 0.014648 & 4.9751 & 0.014345 \\
\hline 668.6071 & $4.4836 \mathrm{e}+05$ & 0.012207 & 5.0414 & 0.012599 \\
\hline 668.6071 & $4.4836 \mathrm{e}+05$ & 0.012207 & 5.1077 & 0.013295 \\
\hline 668.6063 & $4.4837 \mathrm{e}+05$ & 0.014648 & 5.1740 & 0.016432 \\
\hline 668.6063 & $4.4837 \mathrm{e}+05$ & 0.021973 & 5.2403 & 0.024452 \\
\hline 668.6063 & $4.4837 \mathrm{e}+05$ & 0.021973 & 5.3066 & 0.025148 \\
\hline 668.6060 & $4.4837 \mathrm{e}+05$ & 0.024414 & 5.3729 & 0.028285 \\
\hline 668.6061 & $4.4837 \mathrm{e}+05$ & 0.034180 & 5.4392 & 0.038747 \\
\hline 668.6062 & $4.4837 \mathrm{e}+05$ & 0.034180 & 5.5055 & 0.039442 \\
\hline 668.6051 & $4.4837 \mathrm{e}+05$ & 0.039062 & 5.5718 & 0.045020 \\
\hline 668.6052 & $4.4837 \mathrm{e}+05$ & 0.024414 & 5.6381 & 0.031068 \\
\hline & & & & \\
\hline
\end{tabular}

Table B.2 Raw data for Figure 3.16(b), iodine fluorescence about He I line.

\begin{tabular}{|l|l|l|l|l|}
\hline $\begin{array}{l}\text { Wavelength } \\
(\mathrm{nm})\end{array}$ & $\begin{array}{l}\text { Freqency } \\
(\mathrm{GHz})\end{array}$ & $\begin{array}{l}\text { Iodine } \\
\text { fluorescence } \\
\text { signal (arbitrary } \\
\text { units) }\end{array}$ & $\begin{array}{l}\text { Frequency } \\
\text { shift }\left(v-v_{0}\right) \\
(\mathrm{GHz})\end{array}$ & $\begin{array}{l}\text { Detrended } \\
\text { iodine } \\
\text { fluorescence } \\
\text { signal (arb. } \\
\text { units) }\end{array}$ \\
\hline 668.0072 & 448767.62 & 0.52734 & -4.8602 & 0.31067 \\
\hline 668.0072 & 448767.62 & 0.41260 & -4.7954 & 0.19749 \\
\hline 668.0071 & 448767.66 & 0.31494 & -4.7306 & 0.10139 \\
\hline 668.0069 & 448767.81 & 0.25391 & -4.6658 & 0.041911 \\
\hline 668.0069 & 448767.81 & 0.23926 & -4.6010 & 0.028822 \\
\hline 668.0070 & 448767.78 & 0.25146 & -4.5362 & 0.042587 \\
\hline 668.0068 & 448767.87 & 0.26611 & -4.4714 & 0.058793 \\
\hline 668.0067 & 448768.00 & 0.32227 & -4.4066 & 0.11650 \\
\hline 668.0065 & 448768.06 & 0.44434 & -4.3418 & 0.24013 \\
\hline 668.0067 & 448768.00 & 0.60791 & -4.2770 & 0.40527 \\
\hline 668.0065 & 448768.09 & 0.77637 & -4.2122 & 0.57528 \\
\hline 668.0065 & 448768.06 & 0.80078 & -4.1474 & 0.60125 \\
\hline 668.0065 & 448768.06 & 1.1035 & -4.0826 & 0.90555 \\
\hline 668.0070 & 448767.78 & 1.4063 & -4.0178 & 1.2098 \\
\hline 668.0069 & 448767.81 & 1.6187 & -3.9530 & 1.4238 \\
\hline 668.0069 & 448767.81 & 1.7212 & -3.8882 & 1.5279 \\
\hline 668.0062 & 448768.31 & 1.7163 & -3.8234 & 1.5246 \\
\hline 668.0061 & 448768.34 & 1.7358 & -3.7586 & 1.5457 \\
\hline 668.0062 & 448768.31 & 1.6968 & -3.6938 & 1.5082 \\
\hline 668.0062 & 448768.28 & 1.5601 & -3.6290 & 1.3730 \\
\hline 668.0061 & 448768.34 & 1.3525 & -3.5642 & 1.1670 \\
\hline 668.0061 & 448768.34 & 1.3184 & -3.4994 & 1.1344 \\
\hline 668.0042 & 448769.63 & 0.94238 & -3.4346 & 0.76000 \\
\hline 668.0042 & 448769.66 & 0.63965 & -3.3698 & 0.45882 \\
\hline 668.0042 & 448769.63 & 0.46143 & -3.3050 & 0.28216 \\
\hline & & & & \\
\hline
\end{tabular}




\begin{tabular}{|c|c|c|c|c|}
\hline 668.0042 & 448769.63 & 0.38574 & -3.2402 & 0.20803 \\
\hline 668.0042 & 448769.63 & 0.36865 & -3.1754 & 0.19250 \\
\hline 668.0041 & 448769.72 & 0.36865 & -3.1106 & 0.19406 \\
\hline 668.0042 & 448769.63 & 0.38330 & -3.0458 & 0.21027 \\
\hline 668.0037 & 448770.00 & 0.39795 & -2.9810 & 0.22647 \\
\hline 668.0037 & 448770.00 & 0.44922 & -2.9162 & 0.27930 \\
\hline 668.0034 & 448770.16 & 0.48584 & -2.8514 & 0.31748 \\
\hline 668.0038 & 448769.91 & 0.49805 & -2.7866 & 0.33125 \\
\hline 668.0037 & 448769.97 & 0.53223 & -2.7218 & 0.36698 \\
\hline 668.0039 & 448769.84 & 0.56397 & -2.6570 & 0.40028 \\
\hline 668.0031 & 448770.37 & 0.60547 & -2.5922 & 0.44334 \\
\hline 668.0029 & 448770.50 & 0.64697 & -2.5274 & 0.48640 \\
\hline 668.0031 & 448770.37 & 0.65674 & -2.4626 & 0.49773 \\
\hline 668.0031 & 448770.37 & 0.69336 & -2.3978 & 0.53591 \\
\hline 668.0032 & 448770.28 & 0.74463 & -2.3330 & 0.58874 \\
\hline 668.0031 & 448770.37 & 0.80566 & -2.2682 & 0.65133 \\
\hline 668.0032 & 448770.31 & 0.85205 & -2.2034 & 0.69927 \\
\hline 668.0027 & 448770.63 & 0.89844 & -2.1386 & 0.74722 \\
\hline 668.0026 & 448770.69 & 0.90576 & -2.0738 & 0.75610 \\
\hline 668.0027 & 448770.63 & 0.93994 & -2.0090 & 0.79184 \\
\hline 668.0029 & 448770.50 & 0.93750 & -1.9442 & 0.79096 \\
\hline 668.0029 & 448770.50 & 0.93994 & -1.8794 & 0.79496 \\
\hline 668.0030 & 448770.44 & 0.92041 & -1.8146 & 0.77698 \\
\hline 668.0023 & 448770.94 & 0.91553 & -1.7498 & 0.77366 \\
\hline 668.0023 & 448770.91 & 0.89600 & -1.6850 & 0.75569 \\
\hline 668.0023 & 448770.94 & 0.85937 & -1.6202 & 0.72062 \\
\hline 668.0026 & 448770.69 & 0.80810 & -1.5554 & 0.67091 \\
\hline 668.0026 & 448770.72 & 0.73730 & -1.4906 & 0.60167 \\
\hline 668.0024 & 448770.84 & 0.70801 & -1.4258 & 0.57393 \\
\hline 668.0020 & 448771.09 & 0.62500 & -1.3610 & 0.49248 \\
\hline 668.0020 & 448771.09 & 0.50293 & -1.2962 & 0.37197 \\
\hline 668.0018 & 448771.28 & 0.38330 & -1.2314 & 0.25390 \\
\hline 668.0018 & 448771.22 & 0.29053 & -1.1666 & 0.16268 \\
\hline 668.0018 & 448771.22 & 0.29541 & -1.1018 & 0.16913 \\
\hline 668.0020 & 448771.16 & 0.20996 & -1.0370 & 0.085235 \\
\hline 668.0018 & 448771.28 & 0.14893 & -0.97220 & 0.025759 \\
\hline 668.0013 & 448771.59 & 0.11475 & -0.90740 & -0.0068631 \\
\hline 668.0012 & 448771.69 & 0.10010 & -0.84260 & -0.019953 \\
\hline 668.0013 & 448771.59 & 0.087891 & -0.77780 & -0.030601 \\
\hline 668.0015 & 448771.47 & 0.090332 & -0.71300 & -0.026602 \\
\hline 668.0015 & 448771.44 & 0.085449 & -0.64820 & -0.029927 \\
\hline 668.0016 & 448771.34 & 0.080566 & -0.58340 & -0.033251 \\
\hline 668.0007 & 448772.00 & 0.075684 & -0.51860 & -0.036575 \\
\hline 668.0008 & 448771.94 & 0.065918 & -0.45380 & -0.044782 \\
\hline 668.0006 & 448772.03 & 0.068359 & -0.38900 & -0.040783 \\
\hline 668.0010 & 448771.75 & 0.068359 & -0.32420 & -0.039225 \\
\hline 668.0010 & 448771.81 & 0.061035 & -0.25940 & -0.044990 \\
\hline 668.0011 & 448771.72 & 0.061035 & -0.19460 & -0.043432 \\
\hline 668.0001 & 448772.37 & 0.068359 & -0.12980 & -0.034550 \\
\hline 668.0001 & 448772.41 & 0.068359 & -0.065000 & -0.032991 \\
\hline 668.0001 & 448772.41 & 0.073242 & -0.00020000 & -0.026550 \\
\hline 668.0001 & 448772.37 & 0.080566 & 0.064600 & -0.017667 \\
\hline 668.0006 & 448772.03 & 0.078125 & 0.12940 & -0.018550 \\
\hline
\end{tabular}




\begin{tabular}{|c|c|c|c|c|}
\hline 668.0006 & 448772.03 & 0.083008 & 0.19420 & -0.012109 \\
\hline 668.0007 & 448772.00 & 0.078125 & 0.25900 & -0.015433 \\
\hline 667.9996 & 448772.75 & 0.080566 & 0.32380 & -0.011434 \\
\hline 667.9996 & 448772.75 & 0.092773 & 0.38860 & 0.0023314 \\
\hline 667.9996 & 448772.75 & 0.095215 & 0.45340 & 0.0063318 \\
\hline 667.9998 & 448772.62 & 0.095215 & 0.51820 & 0.0078902 \\
\hline 667.9999 & 448772.53 & 0.10742 & 0.58300 & 0.021656 \\
\hline 667.9999 & 448772.53 & 0.11231 & 0.64780 & 0.028097 \\
\hline 667.9991 & 448773.03 & 0.12939 & 0.71260 & 0.046745 \\
\hline 667.9992 & 448773.00 & 0.13672 & 0.77740 & 0.055628 \\
\hline 667.9991 & 448773.03 & 0.15381 & 0.84220 & 0.074276 \\
\hline 667.9991 & 448773.06 & 0.15625 & 0.90700 & 0.078275 \\
\hline 667.9994 & 448772.88 & 0.15381 & 0.97180 & 0.077393 \\
\hline 667.9993 & 448772.91 & 0.16602 & 1.0366 & 0.091158 \\
\hline 667.9993 & 448772.91 & 0.16846 & 1.1014 & 0.095158 \\
\hline 667.9985 & 448773.47 & 0.18066 & 1.1662 & 0.10892 \\
\hline 667.9983 & 448773.59 & 0.18555 & 1.2310 & 0.11536 \\
\hline 667.9984 & 448773.56 & 0.19043 & 1.2958 & 0.12181 \\
\hline 667.9991 & 448773.06 & 0.18799 & 1.3606 & 0.12092 \\
\hline 667.9990 & 448773.16 & 0.19287 & 1.4254 & 0.12736 \\
\hline 667.9991 & 448773.06 & 0.18311 & 1.4902 & 0.11916 \\
\hline 667.9982 & 448773.66 & 0.18799 & 1.5550 & 0.12560 \\
\hline 667.9980 & 448773.81 & 0.17578 & 1.6198 & 0.11495 \\
\hline 667.9980 & 448773.78 & 0.18066 & 1.6846 & 0.12139 \\
\hline 667.9982 & 448773.66 & 0.16602 & 1.7494 & 0.10830 \\
\hline 667.9983 & 448773.59 & 0.13428 & 1.8142 & 0.078120 \\
\hline 667.9983 & 448773.59 & 0.11231 & 1.8790 & 0.057706 \\
\hline 667.9971 & 448774.37 & 0.090332 & 1.9438 & 0.037291 \\
\hline 667.9972 & 448774.34 & 0.092773 & 2.0086 & 0.041291 \\
\hline 667.9973 & 448774.25 & 0.078125 & 2.0734 & 0.028201 \\
\hline 667.9973 & 448774.25 & 0.068359 & 2.1382 & 0.019994 \\
\hline 667.9977 & 448774.00 & 0.065918 & 2.2030 & 0.019111 \\
\hline 667.9977 & 448774.00 & 0.063477 & 2.2678 & 0.018228 \\
\hline 667.9977 & 448774.00 & 0.063477 & 2.3326 & 0.019787 \\
\hline 667.9966 & 448774.75 & 0.083008 & 2.3974 & 0.040876 \\
\hline 667.9966 & 448774.72 & 0.063477 & 2.4622 & 0.022903 \\
\hline 667.9967 & 448774.66 & 0.068359 & 2.5270 & 0.029344 \\
\hline 667.9969 & 448774.50 & 0.058594 & 2.5918 & 0.021137 \\
\hline 667.9970 & 448774.47 & 0.048828 & 2.6566 & 0.012930 \\
\hline 667.9969 & 448774.50 & 0.041504 & 2.7214 & 0.0071639 \\
\hline 667.9958 & 448775.28 & 0.031738 & 2.7862 & -0.0010437 \\
\hline 667.9958 & 448775.25 & 0.026855 & 2.8510 & -0.0043683 \\
\hline 667.9958 & 448775.28 & 0.014648 & 2.9158 & -0.015017 \\
\hline 667.9961 & 448775.09 & 0.017090 & 2.9806 & -0.011017 \\
\hline 667.9961 & 448775.09 & 0.0073240 & 3.0454 & -0.019224 \\
\hline 667.9961 & 448775.09 & 0.0097660 & 3.1102 & -0.015224 \\
\hline 667.9953 & 448775.63 & 0.0000 & 3.1750 & -0.023431 \\
\hline 667.9954 & 448775.53 & 0.0097660 & 3.2398 & -0.012107 \\
\hline 667.9954 & 448775.56 & 0.0097660 & 3.3046 & -0.010549 \\
\hline 667.9951 & 448775.75 & 0.012207 & 3.3694 & -0.0065493 \\
\hline 667.9955 & 448775.44 & 0.019531 & 3.4342 & 0.0023331 \\
\hline 667.9955 & 448775.44 & 0.034180 & 3.4990 & 0.018540 \\
\hline 667.9955 & 448775.50 & 0.048828 & 3.5638 & 0.034747 \\
\hline
\end{tabular}




\begin{tabular}{|l|c|c|c|c|}
\hline 667.9944 & 448776.22 & 0.065918 & 3.6286 & 0.053395 \\
\hline 667.9942 & 448776.34 & 0.063477 & 3.6934 & 0.052513 \\
\hline 667.9942 & 448776.34 & 0.080566 & 3.7582 & 0.071160 \\
\hline 667.9949 & 448775.91 & 0.095215 & 3.8230 & 0.087367 \\
\hline 667.9949 & 448775.91 & 0.11475 & 3.8878 & 0.10846 \\
\hline 667.9948 & 448775.94 & 0.13428 & 3.9526 & 0.12955 \\
\hline 667.9937 & 448776.69 & 0.12695 & 4.0174 & 0.12378 \\
\hline 667.9938 & 448776.59 & 0.12939 & 4.0822 & 0.12778 \\
\hline 667.9937 & 448776.69 & 0.13184 & 4.1470 & 0.13178 \\
\hline 667.9939 & 448776.56 & 0.12939 & 4.2118 & 0.13090 \\
\hline 667.9943 & 448776.28 & 0.11475 & 4.2766 & 0.11781 \\
\hline 667.9944 & 448776.22 & 0.12207 & 4.3414 & 0.12669 \\
\hline 667.9933 & 448776.97 & 0.12207 & 4.4062 & 0.12825 \\
\hline 667.9933 & 448776.94 & 0.13428 & 4.4710 & 0.14201 \\
\hline 667.9934 & 448776.87 & 0.18555 & 4.5358 & 0.19484 \\
\hline 667.9933 & 448776.94 & 0.23437 & 4.6006 & 0.24523 \\
\hline 667.9935 & 448776.84 & 0.30762 & 4.6654 & 0.32003 \\
\hline 667.9934 & 448776.87 & 0.30518 & 4.7302 & 0.31915 \\
\hline 667.9933 & 448776.94 & 0.36865 & 4.7950 & 0.38418 \\
\hline 667.9926 & 448777.41 & 0.44922 & 4.8598 & 0.46631 \\
\hline 667.9924 & 448777.59 & 0.53955 & 4.9246 & 0.55820 \\
\hline 667.9926 & 448777.47 & 0.62744 & 4.9894 & 0.64764 \\
\hline
\end{tabular}

Table B.3 Raw data for Figure 3.16(c), iodine fluorescence about Ar I line.

\begin{tabular}{|l|l|c|c|c|}
\hline $\begin{array}{l}\text { Wavelength } \\
(\mathrm{nm})\end{array}$ & $\begin{array}{l}\text { Freqency } \\
(\mathrm{GHz})\end{array}$ & $\begin{array}{l}\text { Iodine } \\
\text { fluorescence } \\
\text { signal } \\
\text { arbitrary } \\
\text { units })\end{array}$ & $\begin{array}{l}\text { Frequency } \\
\text { shift }\left(v-v_{0}\right)\end{array}$ & $\begin{array}{l}\text { Detrended } \\
\text { iodine } \\
\text { fluorescence } \\
\text { signal (arb. } \\
\text { units })\end{array}$ \\
\hline 667.9208 & 448825.66 & 0.19287 & -5.1550 & 0.015662 \\
\hline 667.9207 & 448825.78 & 0.18555 & -5.0906 & 0.0095464 \\
\hline 667.9206 & 448825.81 & 0.19775 & -5.0262 & 0.022962 \\
\hline 667.9207 & 448825.78 & 0.18311 & -4.9618 & 0.0095206 \\
\hline 667.9208 & 448825.66 & 0.18799 & -4.8974 & 0.015612 \\
\hline 667.9206 & 448825.81 & 0.18311 & -4.8330 & 0.011937 \\
\hline 667.9203 & 448825.97 & 0.18555 & -4.7686 & 0.015587 \\
\hline 667.9203 & 448825.97 & 0.18555 & -4.7042 & 0.016795 \\
\hline 667.9203 & 448826.00 & 0.18311 & -4.6398 & 0.015561 \\
\hline 667.9203 & 448825.97 & 0.18555 & -4.5754 & 0.019211 \\
\hline 667.9202 & 448826.09 & 0.17822 & -4.5110 & 0.013095 \\
\hline 667.9202 & 448826.09 & 0.17822 & -4.4466 & 0.014303 \\
\hline 667.9202 & 448826.06 & 0.18311 & -4.3822 & 0.020393 \\
\hline 667.9199 & 448826.31 & 0.19775 & -4.3178 & 0.036250 \\
\hline 667.9199 & 448826.31 & 0.19043 & -4.2534 & 0.030134 \\
\hline 667.9199 & 448826.31 & 0.19775 & -4.1890 & 0.038667 \\
\hline 667.9198 & 448826.34 & 0.21973 & -4.1246 & 0.061848 \\
\hline 667.9198 & 448826.34 & 0.22949 & -4.0602 & 0.072821 \\
\hline 667.9199 & 448826.31 & 0.26123 & -3.9958 & 0.10577 \\
\hline 667.9193 & 448826.72 & 0.26367 & -3.9314 & 0.10942 \\
\hline 667.9194 & 448826.59 & 0.28565 & -3.8670 & 0.13260 \\
\hline 667.9193 & 448826.72 & 0.32959 & -3.8026 & 0.17775 \\
\hline 667.9194 & 448826.62 & 0.38330 & -3.7382 & 0.23267 \\
\hline 667.9193 & 448826.66 & 0.44678 & -3.6738 & 0.29735 \\
\hline & & & & \\
\hline
\end{tabular}




\begin{tabular}{|c|c|c|c|c|}
\hline 667.9196 & 448826.50 & 0.45898 & -3.6094 & 0.31077 \\
\hline 667.9190 & 448826.88 & 0.54443 & -3.5450 & 0.39743 \\
\hline 667.9190 & 448826.88 & 0.65185 & -3.4806 & 0.50606 \\
\hline 667.9190 & 448826.88 & 0.74951 & -3.4162 & 0.60492 \\
\hline 667.9190 & 448826.88 & 0.82764 & -3.3518 & 0.68425 \\
\hline 667.9188 & 448827.00 & 0.83496 & -3.2874 & 0.69279 \\
\hline 667.9187 & 448827.09 & 0.88135 & -3.2230 & 0.74038 \\
\hline 667.9188 & 448827.00 & 0.90820 & -3.1586 & 0.76844 \\
\hline 667.9185 & 448827.25 & 0.89600 & -3.0942 & 0.75745 \\
\hline 667.9185 & 448827.25 & 0.87647 & -3.0298 & 0.73912 \\
\hline 667.9184 & 448827.28 & 0.82520 & -2.9654 & 0.68906 \\
\hline 667.9183 & 448827.31 & 0.83008 & -2.9010 & 0.69515 \\
\hline 667.9183 & 448827.37 & 0.78857 & -2.8366 & 0.65486 \\
\hline 667.9183 & 448827.37 & 0.75440 & -2.7722 & 0.62189 \\
\hline 667.9179 & 448827.62 & 0.69092 & -2.7078 & 0.55962 \\
\hline 667.9178 & 448827.66 & 0.60547 & -2.6434 & 0.47538 \\
\hline 667.9179 & 448827.62 & 0.59326 & -2.5790 & 0.46438 \\
\hline 667.9179 & 448827.62 & 0.50537 & -2.5146 & 0.37769 \\
\hline 667.9179 & 448827.62 & 0.40527 & -2.4502 & 0.27880 \\
\hline 667.9178 & 448827.66 & 0.33691 & -2.3858 & 0.21165 \\
\hline 667.9174 & 448827.97 & 0.27100 & -2.3214 & 0.14694 \\
\hline 667.9174 & 448827.97 & 0.27588 & -2.2570 & 0.15303 \\
\hline 667.9174 & 448827.97 & 0.23437 & -2.1926 & 0.11274 \\
\hline 667.9174 & 448827.97 & 0.20752 & -2.1282 & 0.087091 \\
\hline 667.9174 & 448827.97 & 0.19043 & -2.0638 & 0.071209 \\
\hline 667.9174 & 448827.94 & 0.18066 & -1.9994 & 0.062651 \\
\hline 667.9174 & 448827.97 & 0.16602 & -1.9350 & 0.049211 \\
\hline 667.9170 & 448828.22 & 0.16357 & -1.8706 & 0.047977 \\
\hline 667.9170 & 448828.22 & 0.16113 & -1.8062 & 0.046744 \\
\hline 667.9168 & 448828.34 & 0.14893 & -1.7418 & 0.035746 \\
\hline 667.9169 & 448828.31 & 0.13672 & $\begin{array}{l}-1.6774 \\
\end{array}$ & 0.024747 \\
\hline 667.9168 & 448828.34 & 0.11719 & -1.6130 & 0.0064237 \\
\hline 667.9169 & 448828.31 & 0.11963 & -1.5486 & 0.010073 \\
\hline 667.9166 & 448828.47 & 0.10742 & -1.4842 & -0.00092611 \\
\hline 667.9165 & 448828.56 & 0.10010 & -1.4198 & -0.0070420 \\
\hline 667.9166 & 448828.50 & 0.090332 & -1.3554 & -0.015600 \\
\hline 667.9164 & 448828.59 & 0.080566 & -1.2910 & -0.024158 \\
\hline 667.9164 & 448828.59 & 0.087891 & -1.2266 & -0.015625 \\
\hline 667.9164 & 448828.59 & 0.073242 & -1.1622 & -0.029066 \\
\hline 667.9163 & 448828.69 & 0.073242 & -1.0978 & -0.027858 \\
\hline 667.9158 & 448829.06 & 0.065918 & -1.0334 & -0.033974 \\
\hline 667.9158 & 448829.00 & 0.078125 & -0.96900 & -0.020558 \\
\hline 667.9158 & 448829.00 & 0.075684 & -0.90460 & -0.021791 \\
\hline 667.9156 & 448829.13 & 0.075684 & -0.84020 & -0.020583 \\
\hline 667.9158 & 448829.06 & 0.075684 & -0.77580 & -0.019375 \\
\hline 667.9159 & 448828.97 & 0.063477 & -0.71140 & -0.030374 \\
\hline 667.9152 & 448829.41 & 0.061035 & -0.64700 & -0.031608 \\
\hline 667.9152 & 448829.41 & 0.061035 & -0.58260 & -0.030400 \\
\hline 667.9152 & 448829.41 & 0.058594 & -0.51820 & -0.031633 \\
\hline 667.9154 & 448829.28 & 0.053711 & -0.45380 & -0.035308 \\
\hline 667.9152 & 448829.47 & 0.061035 & -0.38940 & -0.026776 \\
\hline 667.9150 & 448829.53 & 0.056152 & -0.32500 & -0.030451 \\
\hline 667.9146 & 448829.81 & 0.056152 & -0.26060 & -0.029243 \\
\hline
\end{tabular}




\begin{tabular}{|c|c|c|c|c|}
\hline 667.9146 & 448829.81 & 0.051270 & -0.19620 & -0.032917 \\
\hline 667.9146 & 448829.87 & 0.056152 & -0.13180 & -0.026826 \\
\hline 667.9147 & 448829.75 & 0.051270 & -0.067400 & -0.030500 \\
\hline 667.9146 & 448829.87 & 0.051270 & -0.0030000 & -0.029292 \\
\hline 667.9146 & 448829.81 & 0.041504 & 0.061400 & -0.037850 \\
\hline 667.9144 & 448830.00 & 0.051270 & 0.12580 & -0.026876 \\
\hline 667.9139 & 448830.28 & 0.053711 & 0.19020 & -0.023227 \\
\hline 667.9141 & 448830.16 & 0.048828 & 0.25460 & -0.026902 \\
\hline 667.9141 & 448830.19 & 0.048828 & 0.31900 & -0.025694 \\
\hline 667.9138 & 448830.37 & 0.056152 & 0.38340 & -0.017162 \\
\hline 667.9138 & 448830.37 & 0.065918 & 0.44780 & -0.0061877 \\
\hline 667.9138 & 448830.37 & 0.058594 & 0.51220 & -0.012304 \\
\hline 667.9131 & 448830.81 & 0.073242 & 0.57660 & 0.0035524 \\
\hline 667.9133 & 448830.72 & 0.087891 & 0.64100 & 0.019410 \\
\hline 667.9132 & 448830.78 & 0.083008 & 0.70540 & 0.015735 \\
\hline 667.9133 & 448830.72 & 0.10254 & 0.76980 & 0.036474 \\
\hline 667.9131 & 448830.81 & 0.10498 & 0.83420 & 0.040123 \\
\hline 667.9133 & 448830.72 & 0.11475 & 0.89860 & 0.051097 \\
\hline 667.9132 & 448830.78 & 0.11963 & 0.96300 & 0.057188 \\
\hline 667.9127 & 448831.09 & 0.12207 & 1.0274 & 0.060837 \\
\hline 667.9127 & 448831.09 & 0.12939 & 1.0918 & 0.069370 \\
\hline 667.9128 & 448831.06 & 0.12207 & 1.1562 & 0.063253 \\
\hline 667.9125 & 448831.25 & 0.12451 & 1.2206 & 0.066903 \\
\hline 667.9125 & 448831.25 & 0.11475 & 1.2850 & 0.058345 \\
\hline 667.9123 & 448831.37 & 0.11719 & 1.3494 & 0.061995 \\
\hline 667.9117 & 448831.78 & 0.10742 & 1.4138 & 0.053437 \\
\hline 667.9119 & 448831.62 & 0.10010 & 1.4782 & 0.047322 \\
\hline 667.9119 & 448831.62 & 0.090332 & 1.5426 & 0.038764 \\
\hline 667.9119 & 448831.69 & 0.065918 & 1.6070 & 0.015558 \\
\hline 667.9118 & 448831.72 & 0.041504 & 1.6714 & -0.0076482 \\
\hline 667.9118 & 448831.72 & 0.046387 & 1.7358 & -0.0015571 \\
\hline 667.9113 & 448832.03 & 0.043945 & 1.8002 & -0.0027910 \\
\hline 667.9114 & 448832.00 & 0.036621 & 1.8646 & -0.0089070 \\
\hline 667.9111 & 448832.16 & 0.053711 & 1.9290 & 0.0093911 \\
\hline 667.9113 & 448832.03 & 0.034180 & 1.9934 & -0.0089318 \\
\hline 667.9113 & 448832.09 & 0.051270 & 2.0578 & 0.0093663 \\
\hline 667.9113 & 448832.03 & 0.034180 & 2.1222 & -0.0065156 \\
\hline 667.9111 & 448832.16 & 0.034180 & 2.1866 & -0.0053076 \\
\hline 667.9105 & 448832.56 & 0.046387 & 2.2510 & 0.0081075 \\
\hline 667.9106 & 448832.50 & 0.046387 & 2.3154 & 0.0093156 \\
\hline 667.9107 & 448832.44 & 0.041504 & 2.3798 & 0.0056407 \\
\hline 667.9104 & 448832.66 & 0.034180 & 2.4442 & -0.00047525 \\
\hline 667.9104 & 448832.66 & 0.024414 & 2.5086 & -0.0090332 \\
\hline 667.9103 & 448832.69 & 0.041504 & 2.5730 & 0.0092649 \\
\hline 667.9099 & 448833.00 & 0.051270 & 2.6374 & 0.020239 \\
\hline 667.9099 & 448833.00 & 0.087891 & 2.7018 & 0.058068 \\
\hline 667.9100 & 448832.94 & 0.13428 & 2.7662 & 0.10566 \\
\hline 667.9101 & 448832.87 & 0.20508 & 2.8306 & 0.17767 \\
\hline 667.9097 & 448833.09 & 0.20996 & 2.8950 & 0.18376 \\
\hline 667.9098 & 448833.06 & 0.31494 & 2.9594 & 0.28995 \\
\hline 667.9098 & 448833.06 & 0.39307 & 3.0238 & 0.36928 \\
\hline 667.9092 & 448833.44 & 0.48828 & 3.0882 & 0.46571 \\
\hline 667.9092 & 448833.44 & 0.59326 & 3.1526 & 0.57190 \\
\hline
\end{tabular}




\begin{tabular}{|c|c|c|c|c|}
\hline 667.9092 & 448833.44 & 0.61768 & 3.2170 & 0.59752 \\
\hline 667.9089 & 448833.69 & 0.70801 & 3.2814 & 0.68906 \\
\hline 667.9089 & 448833.69 & 0.77393 & 3.3458 & 0.75618 \\
\hline 667.9091 & 448833.56 & 0.78857 & 3.4102 & 0.77204 \\
\hline 667.9084 & 448833.97 & 0.78125 & 3.4746 & 0.76592 \\
\hline 667.9083 & 448834.06 & 0.76904 & 3.5390 & 0.75493 \\
\hline 667.9083 & 448834.06 & 0.72754 & 3.6034 & 0.71463 \\
\hline 667.9081 & 448834.19 & 0.64941 & 3.6678 & 0.63771 \\
\hline 667.9084 & 448834.00 & 0.51270 & 3.7322 & 0.50220 \\
\hline 667.9084 & 448834.00 & 0.36621 & 3.7966 & 0.35693 \\
\hline 667.9077 & 448834.47 & 0.34668 & 3.8610 & 0.33860 \\
\hline 667.9076 & 448834.53 & 0.20019 & 3.9254 & 0.19333 \\
\hline 667.9078 & 448834.37 & 0.10742 & 3.9898 & 0.10176 \\
\hline 667.9077 & 448834.47 & 0.048828 & 4.0542 & 0.044375 \\
\hline 667.9075 & 448834.59 & 0.0097660 & 4.1186 & 0.0065208 \\
\hline 667.9073 & 448834.72 & 0.029297 & 4.1830 & 0.027260 \\
\hline 667.9076 & 448834.53 & 0.017090 & 4.2474 & 0.016261 \\
\hline 667.9070 & 448834.91 & 0.0097660 & 4.3118 & 0.010145 \\
\hline 667.9070 & 448834.91 & 0.0048830 & 4.3762 & 0.0064701 \\
\hline 667.9070 & 448834.91 & 0.012207 & 4.4406 & 0.015002 \\
\hline 667.9069 & 448835.00 & 0.0048830 & 4.5050 & 0.0088863 \\
\hline 667.9069 & 448835.03 & 0.0024410 & 4.5694 & 0.0076524 \\
\hline 667.9069 & 448835.03 & 0.0024410 & 4.6338 & 0.0088605 \\
\hline 667.9061 & 448835.53 & 0.0073240 & 4.6982 & 0.014952 \\
\hline 667.9061 & 448835.53 & 0.0000 & 4.7626 & 0.0088356 \\
\hline 667.9061 & 448835.53 & 0.0024410 & 4.8270 & 0.012485 \\
\hline 667.9062 & 448835.50 & 0.012207 & 4.8914 & 0.023459 \\
\hline 667.9061 & 448835.56 & 0.026855 & 4.9558 & 0.039315 \\
\hline 667.9061 & 448835.53 & 0.036621 & 5.0202 & 0.050289 \\
\hline 667.9061 & 448835.56 & 0.065918 & 5.0846 & 0.080794 \\
\hline 667.9055 & 448835.97 & 0.087891 & 5.1490 & 0.10398 \\
\hline
\end{tabular}


Content-Type: text/plain; charset="us-ascil"

To: Greg Severn <severntengr.wisc.edu>

From: Craig Sansonetti <Craig.Sansonettianist.gov>

Subject: I2 classifications

Content-Iength: 1615

status : $\mathbf{R O}$

Dear Greg.

have calculated the I2 B-X spectrum between 14955 and $14957 \mathrm{~cm}-1$

using the Dunham coefficients from $S$. Gerstenkorn and $P$. Luc, $J$. (1985) in a program that was given to NIST by of the approximately 80 transitions predicted in this region, the following appear most likely to be observed in your spectra.

Classification Wavenuber Severn line (???)

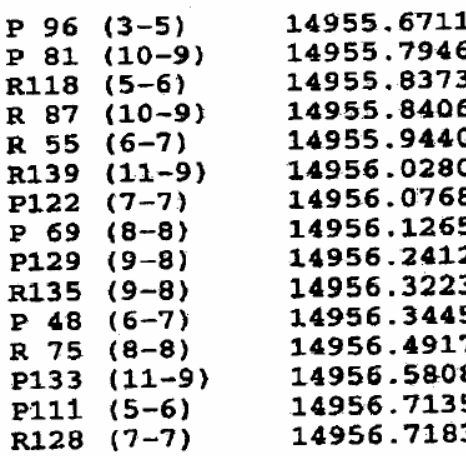

360

538

647

892

987

1280

It appears to me that these possibilities probably account for It appears toctrum. I have tentatively placed all of the features in your spectrum. the lines whose positions you indicated with the classificats just that seem most likely to me ino real science here, just my bost be guess). For several of these, however, there may very well more than one transition contributing to the observed line. definitive identification.

You might want to note that Gerstenkoin and Luc published an rou might warrection to the atlas wavenumbers of $-0.0056 \mathrm{~cm}-1$. additive correction to the atlas wave I think, be applied to both the wavenumbers in the printed atlas and the calculated wavenumbers above.

Figure B.1 Private communication between Craig Sansonetti of NIST and Greg Severn about iodine fluorescence lines. 


\section{Appendix C: International Equipment Transit}

When collaborating with international colleagues, it is often necessary to transport scientific equipment. This process is incredibly complicated and can lead to lots of stress, foul language, and ruined vacations. Because of this, planning very far in advance of the actual trip is essential, though this will not guarantee a problem-free journey. This appendix only gives information about my experiences with international equipment transit. It by no means gives a guide on how to transport equipment easily, but gives warnings of what to avoid and some possible suggestions where further research should be done.

The main issue with transporting scientific equipment is that every country will require you to pay customs fees for anything purchased in another country. If you send equipment to another country, you must prove that it will not stay in that country. When you return the equipment to your country, you have to prove that you owned in that country before sending it to another country. The average traveler does not encounter this problem with their usual belongings because it is accepted that you will not pack your clothes and other personal items, and then leave them strewn across the country to which you are traveling. You are also usually given some maximum amount of items which you can purchase and bring home without having to pay customs. However, once this maximum is exceeded, duty fees are assessed and charged. Scientific equipment, however, does not fall under the personal belonging category and the value usually exceeds the "souvenir" maximum.

We chose to ship the large, heavy, and non-fragile equipment, such as the laser power supply, PMT power supplies, optics mount, etc. In order to deal with the customs 
issue, we obtained a carnet (pronounced "car-nay"), which is similar to a passport except it is used for stuff instead of people. A carnet can be obtained through the Corporation for International Business, which is the ATA Carnet provider for the United States Council for International Business (USCIB). Their website (http://www.atacarnet.com/) contains information on applying for a carnet, carnet fees, countries which accept carnets, etc. The carnet will include a list of the equipment and each item's weight and value. A carnet deposit will be required, based on the total value of the equipment. Because this amount may be very high, there is a waiver of this deposit for government-funded agencies. As a university, we are government funded, though below the amount required for the waiver. We were able to plead our case and obtained the waiver. The carnet fees then were a $\$ 205$ document fee and a $\$ 250$ deposit.

Once a carnet has been obtained for the equipment, the equipment has to be shipped. UPS and FedEx do not handle carnets; DHL "does." We decided to ship our equipment with DHL because they claimed to handle carnets. We were warned by the USCIB personnel that people had problems with DHL and carnets. The most quoted problem was that the equipment was shipped out of the US without the carnet paperwork being filled out by customs. Thus, upon returning to the US, the duty fees were still charged. Unfortunately, we were unable to find another option. (Upon being warned about DHL, I asked what other options there were, and I was told to look under "customs brokers" in the yellow pages. There is no such heading in the yellow pages. Anyway, I would recommend to the reader that they look further into this "customs brokers" field as a more reliable method.) 
I scheduled the packages to be picked up by DHL one week prior to my departure. I asked the driver whether he knew what a carnet was and what he was supposed to do with it. He did not. He called his supervisor for more information, and she seemed to be more knowledgeable of the carnet process. The primary piece of information that she gave was that he needed to place "carnet" stickers on the packages. He took the packages and the carnet. Two days later began a series of confusing phone calls about some sort of shipping problem. After many phone calls to the company, I found out that the packages were sitting in Los Angeles, waiting for the associated carnet paperwork. (Luckily, the "carnet" stickers were on the packages so that they did not get shipped straight to Australia.) After telling several people that I had given the driver the carnet, they informed me that I should have attached it to the package in a document envelope, and that, since the carnet was now lost, we would have to replace it. I made several frantic calls to the USCIB and to Earl (who was on vacation) and was able to get the carnet replaced, which involved another $\$ 455$ fee. There was some discussion that DHL would refund this amount to us. The USCIB FedExed the new carnet to me to sign, and I FedExed it to the DHL (hopefully you find this detail as amusing as I did) customs employee in LA.

The packages arrived, with proper paperwork completed (!) at ANU about two weeks later. The carnet was now in a document envelope attached to the packages. Upon leaving Australia, I repacked the boxes and scheduled a pickup, giving a credit card number to be billed for the shipment (as we had done for the original shipment). I hoped that the Australian DHL employees were a little more up on procedures. (DHL has been an established international company, but had recently purchased Airborne Express in the 
US, which was one of their excuses why the driver was clueless.) Besides, as long as the carnet stayed in the envelope attached to the box, it was going to get seen appropriately by customs. After returning from Australia, the boxes arrived at WVU, but the carnet did not. It must have made it through customs just fine, but was lost somewhere afterwards by DHL. Normally we would have been ecstatic just to get the equipment back, but we were required by the USCIB to return the carnet because it is a federal document, and the $\$ 500$ total deposit would also be returned upon receipt. So several rounds of calls to DHL later, the carnet was found and shipped back to us. Well, actually, it was shipped to the Engineering Department, who kindly sent it over to us. The document was happily returned to USCIB and our deposit was refunded. DHL then proceeded to bill the Foreign Language Department's account for the return shipment. After they refused to pay that bill several times, we were informed of the situation. We informed DHL that we'd be happy to pay the bill, provided it was actually billed to us and only upon receiving the $\$ 455$ discount promised for the lost carnet. Needless to say, this caused much confusion, and, to make a long story short, we have never been billed for the return shipment. (For another DHL incompetence story, see http://www.why-i-would-never-use-dhl.com/.)

For the fragile items, such as the laser head, filters, and PMTs, we purchased a $14.8 " \times 10.4 " \times 6.9$ " Pelican ${ }^{\mathrm{TM}}$ carrying case with customizable foam insert which I carried with me on the plane. In order to prove that we had possession of this equipment prior to leaving the US, I went to the customs office near the Pittsburgh, PA airport. There I filled out a simple form on which I listed the objects. A customs officer filled in all the extra white space and signed the form. When taking the case through security and asked what it was, I answered "scientific equipment." (I figure the word "laser" is not 
likely to go over too well for airplane travel.) Everyone accepted that and only one place asked to look under the items, courteously asking whether he would ruin anything if the case was opened or anything were moved prior to doing so. On the way back into the US, the customs form included a question involving property for business use, which I checked as affirmative. The customs officer asked what it was, to which I again answered "scientific equipment." She asked if I had it with me originally when I left the US, to which I replied in the affirmative, and she let me pass. The simple customs form was never needed.

So for future international travel, anything you can carry with you will be much simpler. One student in the EAPSI program had paid the extra luggage fees to the airline so that she could bring her equipment as baggage. It is recommended that you check with all airlines on which you are booked prior to attempting this method. This may be especially difficult if there is a connection on a smaller plane within either country. However, this might be solved by carrying the baggage internationally, and then having it shipped to your final destination once you arrive. If you do require shipment of equipment, I would recommend looking into customs brokers that will take your packages through customs to ensure that the carnet paperwork is handled correctly. 


\section{Appendix D:Collisional-Radiative Model Code}

The following is the MATLAB ${ }^{\circledR}$ code used to generate the electron energy distribution functions for input to the CR model. The "\%" sign begins a comment.

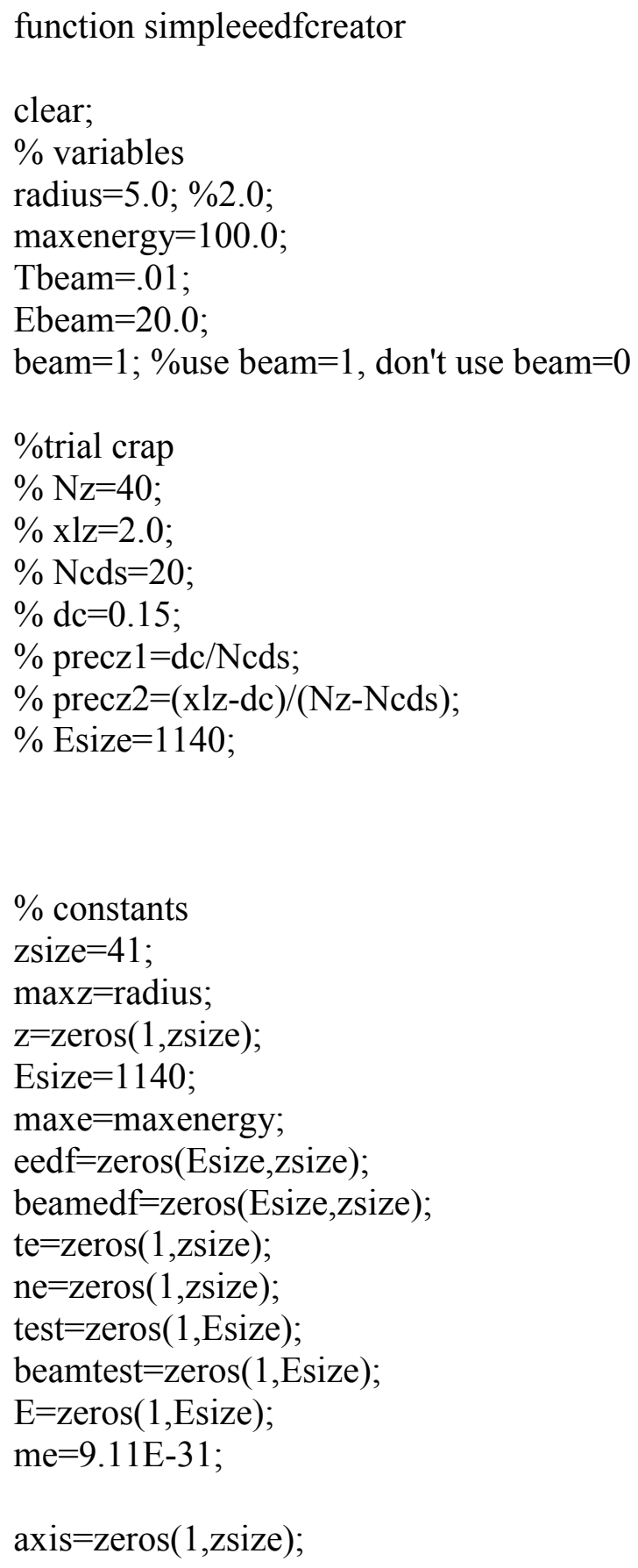




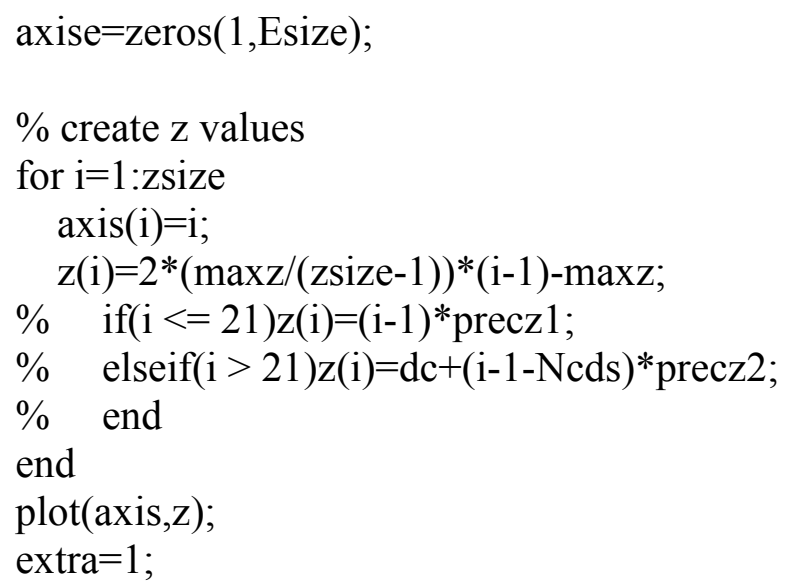




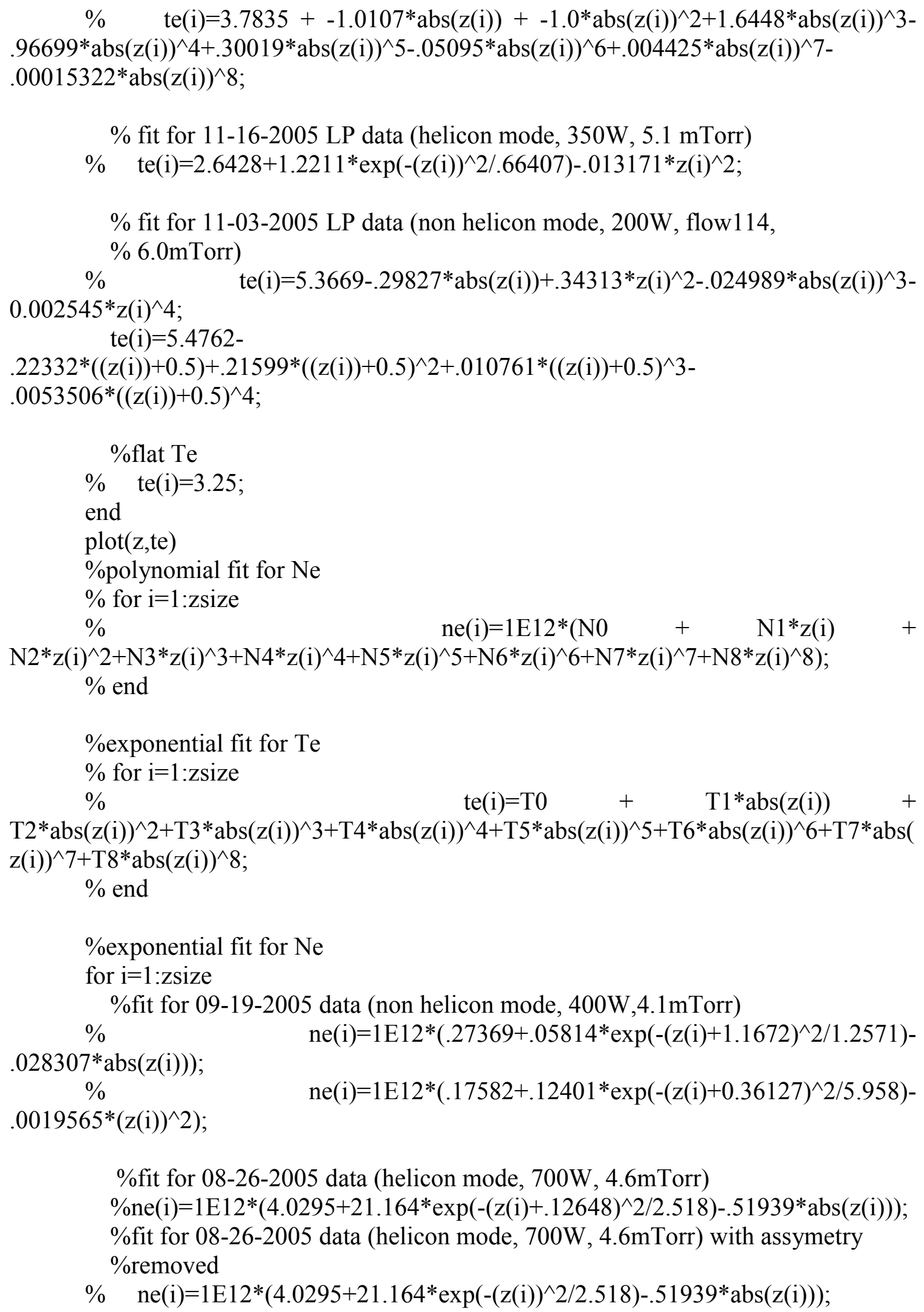




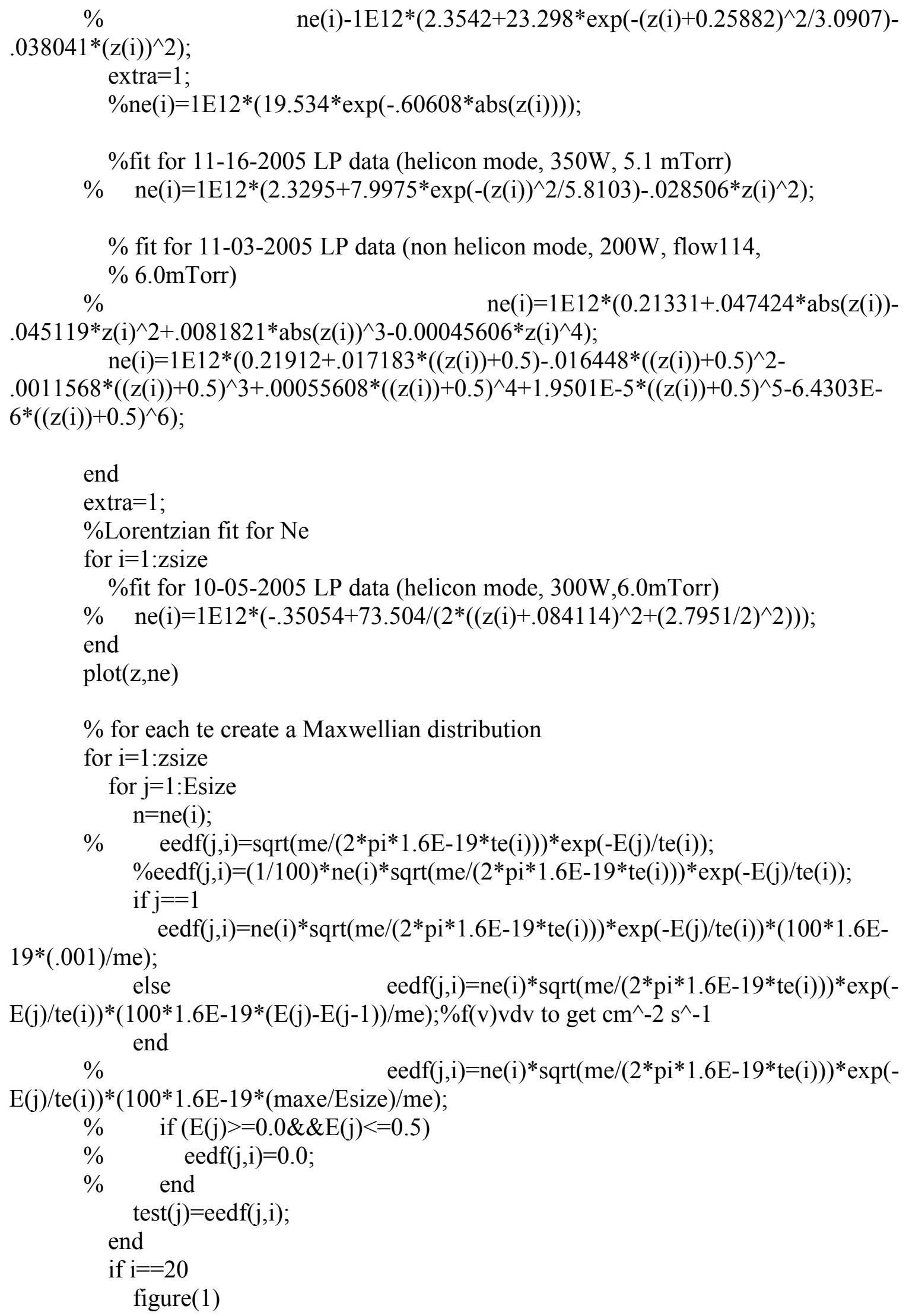




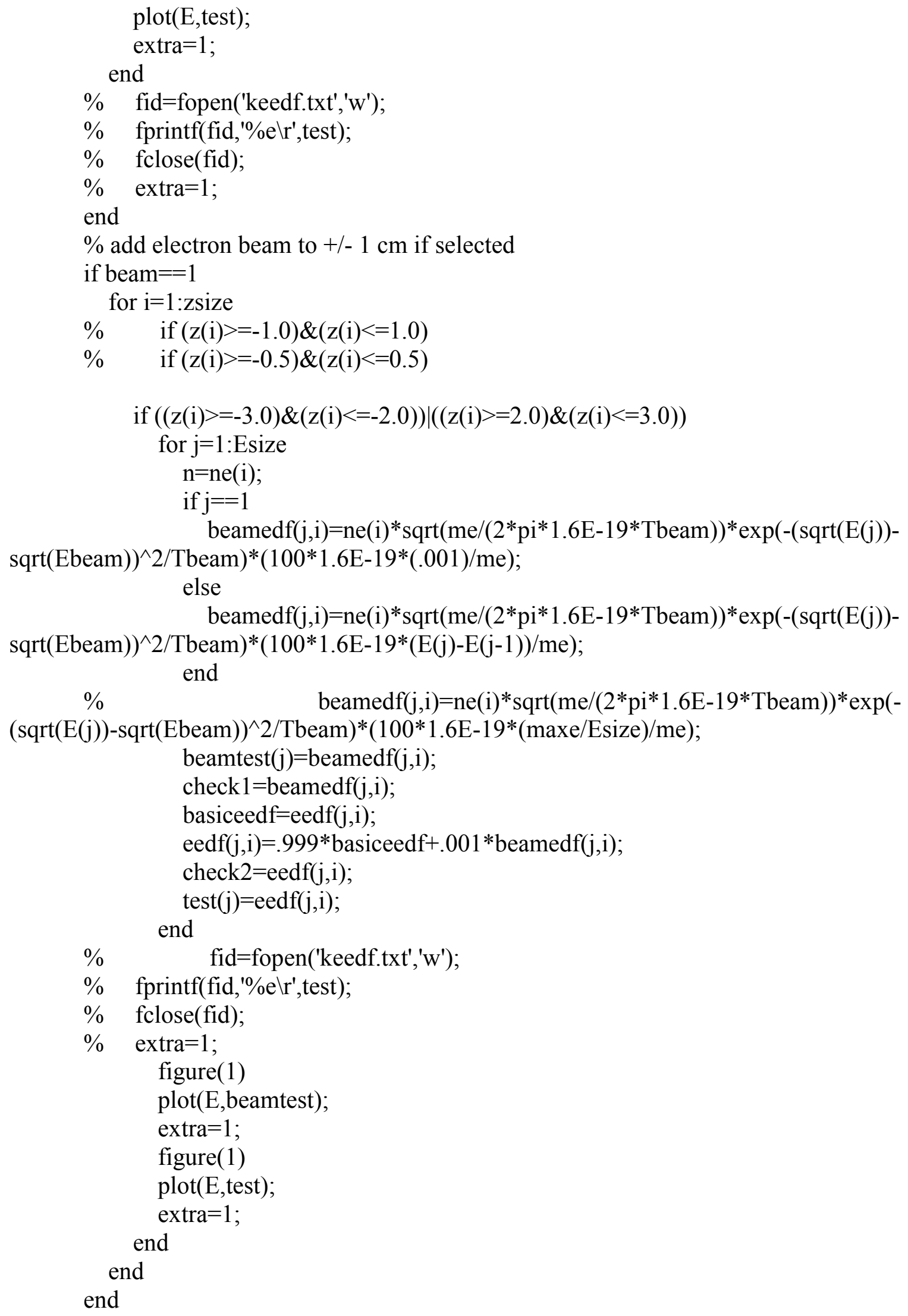


figure(1)

$\operatorname{plot}(\mathrm{E}, \mathrm{test})$;

$\%$ write the eedf's to a file that can be read into Bogaert's code

save eedf.dat eedf -ascii;

$\%$ write ne to a file that can be read into Bogaert's code

save ne.dat ne -ascii;

\%write to a file that can be opened in kgraph

fid=fopen('keedf.txt','w');

fprintf(fid, $\%$ e, ',eedf);

The following is the FORTRAN code used to run the CR model. Comment lines can begin with either a "c" or an "'!".

c Collisional radiative model (1D)

c recieved by Amy Keesee (AMK) from Annemie Bogaerts on 9/17/03

c modified $9 / 22 / 03$ by AMK

c $12 / 2 / 03$

c $\quad 12 / 18 / 03 \mathrm{Ee}(0: \mathrm{NE})$, zero added to force indexing from zero

c that had been causing NaN problems

c 2/17/04 modifications to read eedf from matlab calculations,

c no need for nm or atom edf, no need for Ncds

c 2/17 NE changed from 1200

c $\quad 10 / 11 / 04$ add $\mathrm{r}(0: \mathrm{Nz})$

c $\quad 10 / 12 / 04$ add iz dependence to $x n 0$

c THIS PROGRAM MUST BE IN DOUBLE PRECISION parameter $(\mathrm{Nz}=40, \mathrm{Ncds}=20, \mathrm{Ntot}=65, \mathrm{Nen}=1100, \mathrm{NE}=1139)$ real z(0:Nz), zh( $(0: \mathrm{Nz}), \mathrm{dz}(0: \mathrm{Nz}), \mathrm{dzz}(0: \mathrm{Nz}), \operatorname{pop}(\mathrm{Ntot}, 0: \mathrm{Nz})$,

. $\mathrm{D}(4), \mathrm{k} 2 \mathrm{~b}(4), \mathrm{k} 3 \mathrm{~b}(4), \mathrm{nes}(0: \mathrm{Nz}), \mathrm{ni}(0: \mathrm{Nz}), \mathrm{nm}(0: \mathrm{Nz}), \mathrm{fac}(\mathrm{Ntot})$,

. Eion(Ntot),Eexc(Ntot),aAf(Ntot,Ntot),aP(Ntot,Ntot),gam(Ntot),

- $\mathrm{g}(\mathrm{Ntot}), \mathrm{g} 0(\mathrm{Ntot}), \mathrm{gi}(\mathrm{Ntot}), \mathrm{A}(\mathrm{Ntot}, \mathrm{Ntot})$, popold(Ntot, $0: \mathrm{Nz})$,

. $\operatorname{Esc}(\mathrm{Ntot}, 0: \mathrm{Nz}), \operatorname{Ee}(0: \mathrm{NE}), \mathrm{fe}(0: \mathrm{NE}, 0: \mathrm{Nz}), \operatorname{Ei}(0: \mathrm{NE}), \mathrm{fi}(0: \mathrm{NE}, 0: \mathrm{Nz})$,

. $\mathrm{fa}(0: \mathrm{NE}, 0: \mathrm{Nz})$,

. kione(Ntot,0:Nz),rrec1e(Ntot,0:Nz),rrec2e(Ntot,0:Nz),

. $\operatorname{kexce}($ Ntot,Ntot,0:Nz),kdeexe(Ntot,Ntot,0:Nz),kioni(Ntot,0:Nz),

. $\operatorname{rreci}(\mathrm{Ntot}, 0: \mathrm{Nz}), \mathrm{kexci}(\mathrm{Ntot}, \mathrm{Ntot}, 0: \mathrm{Nz}), \mathrm{kdeexi}(\mathrm{Ntot}, \mathrm{Ntot}, 0: \mathrm{Nz})$,

. kiona(Ntot,0:Nz),rreca(Ntot,0:Nz),kexca(Ntot,Ntot,0:Nz), 
. kdeexa(Ntot,Ntot, $0: \mathrm{Nz})$,kionth(Ntot, $0: \mathrm{Nz}), \operatorname{rrecth}(\mathrm{Ntot}, 0: \mathrm{Nz})$,

. kexcth(Ntot,Ntot,0:Nz),kdeexth(Ntot,Ntot,0:Nz),Kloss(Ntot,0:Nz),

. ai $(4,0: \mathrm{Nz}), \operatorname{ci}(4,0: \mathrm{Nz}), \operatorname{bbi}(4,0: \mathrm{Nz}), \operatorname{beta}(0: \mathrm{Nz}), \operatorname{gamma}(0: \mathrm{Nz})$,

. texce(Ntot),tdeexe(Ntot),texci(Ntot),tdeexi(Ntot),texca(Ntot),

. tdeexa(Ntot), texcth(Ntot),tdeexth(Ntot),trad(Ntot),

. ftote $(0: \mathrm{Nz}), \operatorname{relae}(0: \mathrm{Nz}), \operatorname{ree}(0: \mathrm{Nz})$, rione $(0: \mathrm{Nz}), \operatorname{rtot}(0: \mathrm{Nz})$,

. ftoti $(0: \mathrm{Nz}), \operatorname{rtoti}(0: \mathrm{Nz}), \operatorname{ftota}(0: \mathrm{Nz}), \operatorname{rtota}(0: \mathrm{Nz})$,

. ttione(Ntot),ttrec1e(Ntot),ttrec2e(Ntot),ttexce(Ntot,Ntot),

. ttdeexe(Ntot,Ntot), ttioni(Ntot),ttreci(Ntot),ttexci(Ntot,Ntot),

. ttdeexi(Ntot,Ntot),ttiona(Ntot),ttreca(Ntot),ttexca(Ntot,Ntot),

. ttdeexa(Ntot,Ntot),rtot2(0:Nz),poptot, $\mathrm{r}(0: \mathrm{Nz}), \mathrm{xn} 0(0: \mathrm{Nz})$

parameter $(\mathrm{pi}=3.1415926536)$

character cstr*100

integer $\mathrm{n} 2$ (Ntot)

c input files

c data, necessary for cross sections, trans.probab.

c .dat extension added for levels* files by AMK 12/2/03

$$
\begin{aligned}
& \text { open(unit=1,file='levels1.dat',status='unknown') } \\
& \text { open(unit }=2 \text {, file='levels2.dat',status='unknown') } \\
& \text { open(unit=3, file='levels3.dat',status='unknown') }
\end{aligned}
$$

c EDF of electrons

c $\quad(2 / 17)$ Use file from matlab calculations open(unit $=4$,file $=$ 'eedf.dat',status $=$ 'unknown')

c ne, ni from fit to LP data, file ne.dat written by matlab code open(unit $=8$, file='ne.dat',status $=$ 'unknown')

c output

$\mathrm{c}$ level populations open (unit $=14$, file $=$ 'crlb.dat', status $=$ 'unknown')

c contrib.of prod.-loss processes open(unit $=15$, file $=$ 'cr2b.dat', status $=$ 'unknown')

c coll. processes

open (unit $=16$,file $=$ 'cr3b.dat', status $=$ 'unknown')

open(unit $=17$,file $=$ 'cr4b.dat', status $=$ 'unknown')

open(unit $=18$,file $=$ 'cr5b.dat',status $=$ 'unknown')

! open(unit $=21$,file $=$ 'keedf.txt',status $=$ 'unknown')

open(unit $=22$, file $=$ 'check.txt', status $=$ 'unknown')

$c$ input of data

c general (geometry, precisions, discharge conditions) 
c (2/17) WE NEED OUR OWN VALUES HERE!!!!!!!!

c $\quad \mathrm{xlz}=2.0$

c $\quad \mathrm{xrad}=2.0$

c $\quad \mathrm{dc}=0.15$

c $\quad$ pres $=1000$

c $x$ tgas $=450$

c $\quad \mathrm{xn} 0=3.535 \mathrm{e} 16 *$ pres $/ 1000 * 273 / \mathrm{xtgas}$

c $\quad \mathrm{dt}=1 \mathrm{e}-5$

c $\quad$ precz $1=\mathrm{dc} / \mathrm{Ncds}$

c $\quad$ precz $2=(x l z-d c) /(\mathrm{Nz}-\mathrm{Ncds})$

c $\quad$ xtgas $=300$

c our values

c do $\mathrm{z}=0, \mathrm{xlz}$ but really $\mathrm{r}=-\mathrm{xlz} / 2, \mathrm{xlz} / 2$

$\mathrm{xlz}=10.0$

$\mathrm{xrad}=\mathrm{xlz} / 2.0$

precz $=x l z / F L O A T(N z)$

c pressure in mTorr (use fill(no plasma) pressure from baratron)

! $\quad$ pres $=5.1$

! $\quad$ pres $=4.1$

! $\quad$ pres $=4.6$

pres $=6.0$

c $\quad$ pres $=15.6$

c $\quad x$ tgas is temperature in $\mathrm{K}$

xtgas $=348$

$\mathrm{dt}=1 \mathrm{e}-3$

wid $=3.0$

$\mathrm{wl}=\mathrm{xrad}-(\mathrm{wid} / 2)$

$\mathrm{wr}=\mathrm{xrad}+(\mathrm{wid} / 2)$

c $\quad \mathrm{P}$ is depletion percentage $(11 \%=.11)$ if $\mathrm{P}>.5$ then $\mathrm{dd}=1 /(1-\mathrm{P})$

c if $\mathrm{P}<.5$ then $\mathrm{dd}=1 / \mathrm{P}, \mathrm{da}=1-\mathrm{P}$

$\mathrm{dd}=5.0$

$\mathrm{da}=0.8$

do $6 \mathrm{iz}=0, \mathrm{Nz}$

c flat ground state profile

! $\quad \mathrm{xn0}(\mathrm{iz})=3.535 \mathrm{e} 16 *$ pres $/ 1000 * 273 / \mathrm{xtgas}$

c parabolic profile with depletion $\mathrm{P}$

! $\quad \mathrm{xn} 0(\mathrm{iz})=3.535 \mathrm{e} 16 *$ pres $/ 1000 * 273 / \mathrm{xtgas}^{*}$

! . $\quad((($ precz*iz/xrad-1.0)**2)/dd + da $)$

c profile using LP data for certain params

! $\quad \mathrm{xn} 0(\mathrm{iz})=1 \mathrm{E} 14 *\left(1.25-0.218 * \exp \left(-\left(\right.\right.\right.$ precz $\left.\left.^{*} \mathrm{iz}-5+1.066 \mathrm{E}-7\right) * * 2 / 2.27\right)$

! $\quad+.000444 *\left(\right.$ precz $\left.\left.^{*} \mathrm{iz}-5\right) * * 2\right)$

c profile with depletion of width wid

$\mathrm{zval}=$ precz*iz

if((zval.ge.wl).and.(zval.le.wr))then 


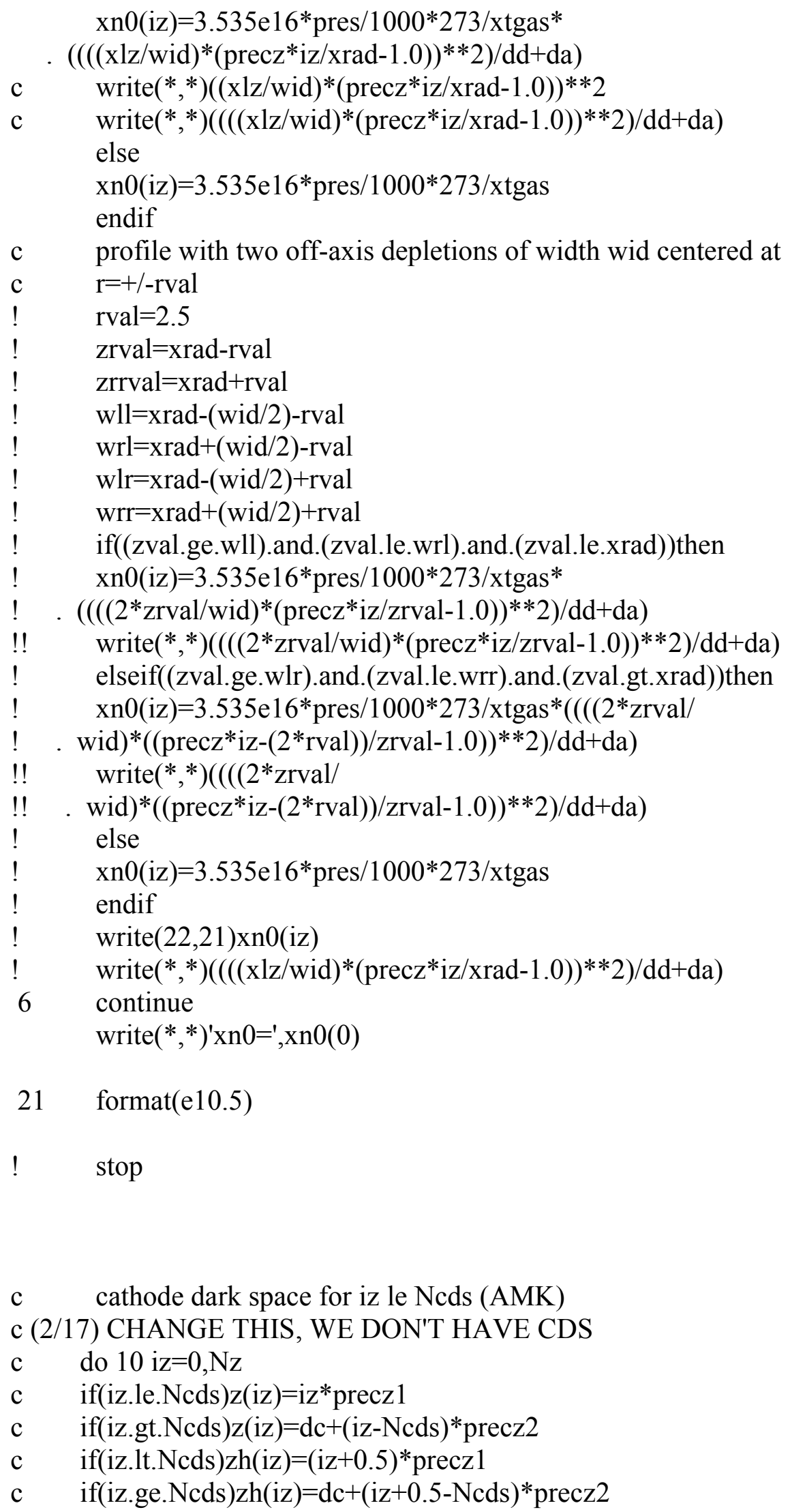




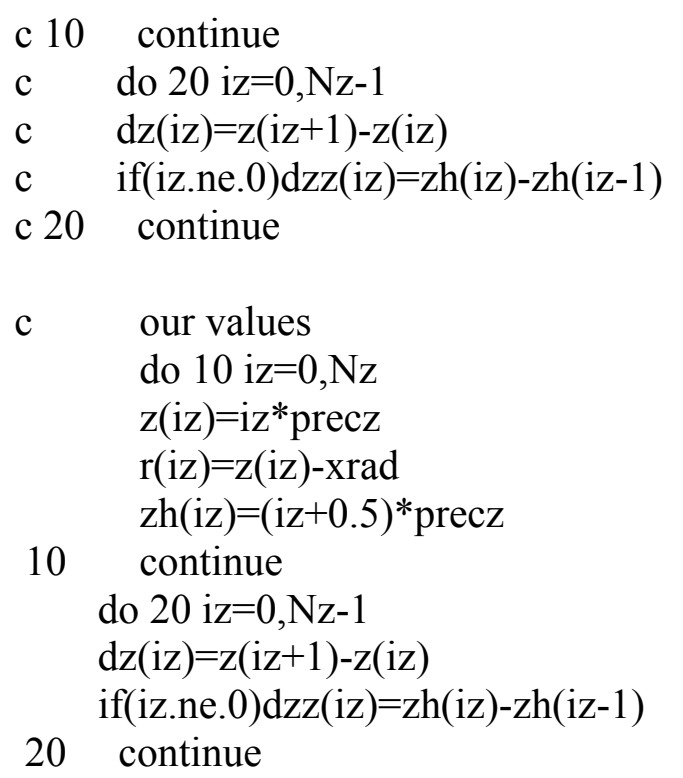

c data for metastables

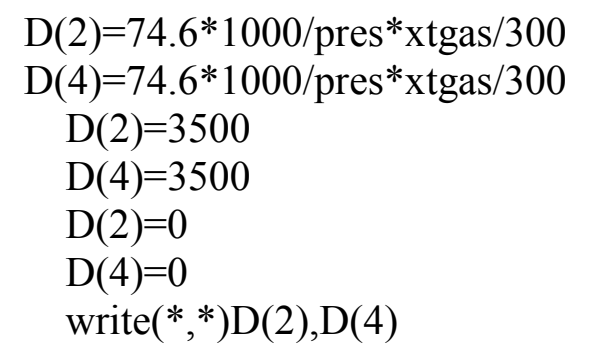

$\mathrm{xkmet}=6.4 \mathrm{e}-10$
$\mathrm{xkpi}=2.36 \mathrm{e}-10$
$\mathrm{k} 2 \mathrm{~b}(2)=2.3 \mathrm{e}-15$
$\mathrm{k} 3 \mathrm{~b}(2)=1.4 \mathrm{e}-32$
$\mathrm{k} 2 \mathrm{~b}(4)=4.3 \mathrm{e}-15$
$\mathrm{k} 3 \mathrm{~b}(4)=1.5 \mathrm{e}-32$

c input of data from files:

c data necessary for cross sections

c read ionization+excitation energy, and degeneracies of the levels:

$\operatorname{read}\left(1,(\mathrm{a} 80)^{\prime}\right) \operatorname{cstr}$

do $30 \mathrm{n}=1$,Ntot

$\operatorname{read}(1,7) n, \operatorname{Eion}(n), \operatorname{Eexc}(n), g(n), g 0(n), g i(n), \operatorname{gam}(n)$

if $(\operatorname{gi}(n)$. eq.6. $) \operatorname{fac}(n)=1.0$

if $($ gi(n).eq.4. $) \operatorname{fac}(n)=0.667$

if $(\mathrm{gi}(\mathrm{n})$.eq. 2 .) $\mathrm{fac}(\mathrm{n})=0.333$

! $\quad$ write $(*, *) n, \operatorname{Eexc}(\mathrm{n})$ 
c read aA $*$ fmn (allowed) and aP (forbidden) coeff.for elec.excit:

c read Amn (trans.probab.):

$\operatorname{read}\left(2,{ }^{\prime}(\mathrm{a} 80)^{\prime}\right) \mathrm{cstr}$

$\operatorname{read}\left(3,,^{\prime}(\mathrm{a} 80)^{\prime}\right) \mathrm{cstr}$

do $35 \mathrm{n}=1$,Ntot

do $35 \mathrm{~m}=\mathrm{n}+1$, Ntot

$\operatorname{read}(2,8) n, m, a A f(n, m), a P(n, m)$

$\operatorname{read}(3,9) n, m, A(n, m)$

35 continue

7 format(i2,2(1x,f6.3),1x,f5.0,1x,f5.3,1x,f2.0,1x,f8.4)

8 format(2(1x,i2),2(1x,e8.2))

9 format $(2(1 \mathrm{x}, \mathrm{i} 2), 1 \mathrm{x}, \mathrm{e} 8.2)$

c EDF of electrons new version

$$
\mathrm{iEe}=-1
$$

do $40 \mathrm{i}=0, \mathrm{NE}$

$\mathrm{iEe}=\mathrm{iEe}+1$

if(iEe.lt.11)Ee(iEe)=iEe*.001

if $((\mathrm{iEe} . g e .11)$.and.(iEe.lt.20)) $\mathrm{Ee}(\mathrm{iEe})=(\mathrm{iEe}-9) * .01$

if $((\mathrm{iEe} . g e .20)$.and.(iEe.lt.29) $) \mathrm{Ee}(\mathrm{iEe})=(\mathrm{iEe}-18)^{*} .1$

if $((\mathrm{iEe} . g e .29)$. and.(iEe.lt.43) $) \mathrm{Ee}(\mathrm{iEe})=(\mathrm{iEe}-28) * .2+1.0$

if $(\mathrm{iEe} . \mathrm{ge} .43) \mathrm{Ee}(\mathrm{iEe})=(\mathrm{iEe}-43)+4.0$

$\operatorname{read}(4,19)(\mathrm{fe}(\mathrm{iEe}, \mathrm{iz}), \mathrm{iz}=0, \mathrm{Nz})$

! if(iEe.eq.15)write $(*, *)$ fe(iEe,15)

! if(iEe.eq.15)stop

40 continue

19 format(41(e16.13))

!c EDF of electrons (modified 2/17)

!! $\operatorname{maxE}=1100.0$

! $\quad \max E=100.0$

! $\quad \operatorname{precE}=\max \mathrm{E} / \mathrm{FLOAT}(\mathrm{NE})$

! $\quad \mathrm{iEe}=-1$

! do $40 \mathrm{i}=0, \mathrm{NE}$

! $\quad \mathrm{iEe}=\mathrm{iEe}+1$

! $\quad \mathrm{Ee}(\mathrm{iEe})=\operatorname{precE}^{*}(\mathrm{iEe}+1)$

! $\quad \operatorname{read}(4,19)(\mathrm{fe}(\mathrm{iEe}, \mathrm{iz}), \mathrm{iz}=0, \mathrm{Nz})$

! 40 continue

!

! 19 format(41(e16.13)) 


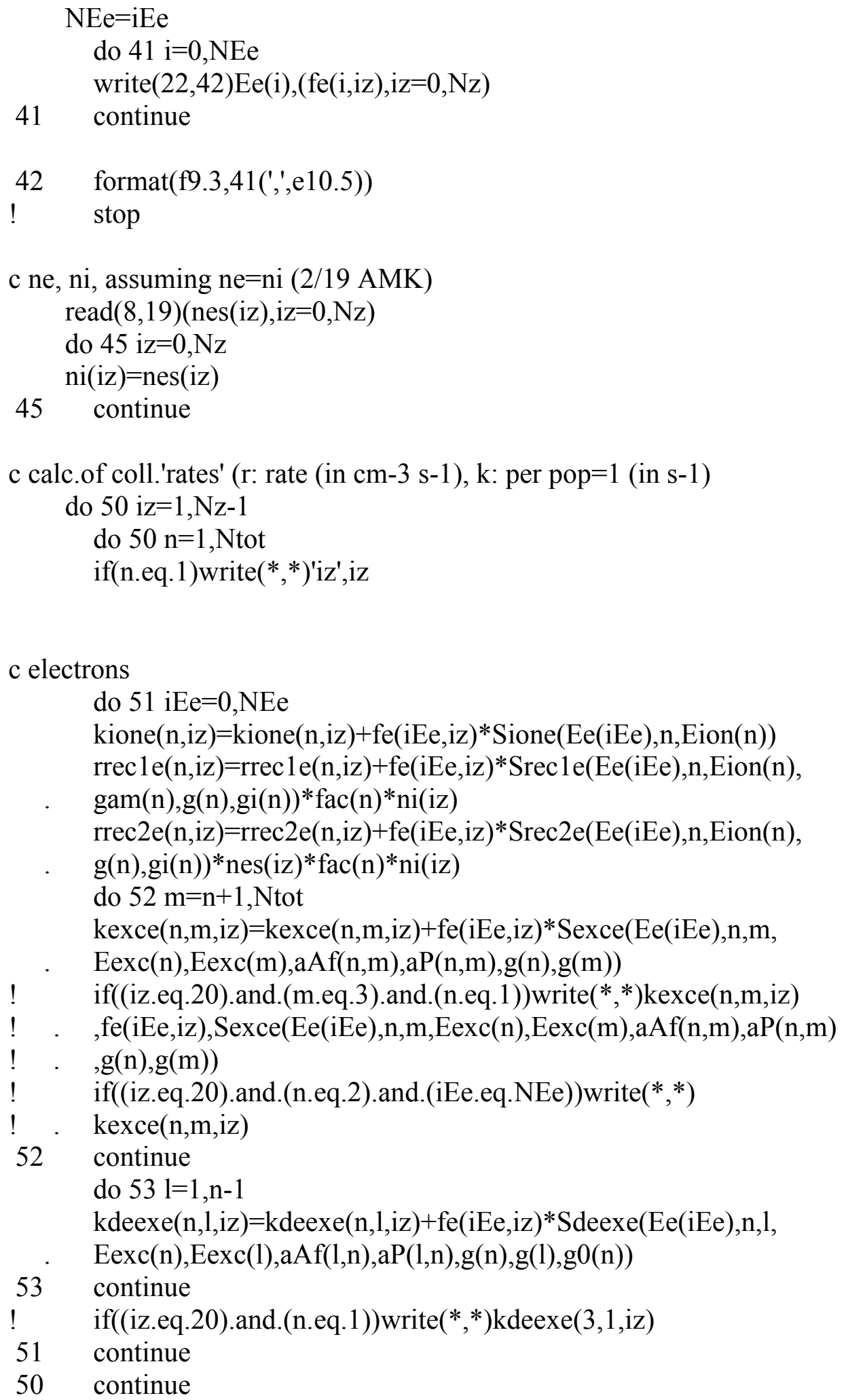


$\mathrm{c}$ thermalized atoms $(\mathrm{E}=0.06 \mathrm{eV}->\mathrm{v}=5.36 \mathrm{e} 4 \mathrm{~cm} / \mathrm{s})(\mathrm{k}$ : per pop=1, in $\mathrm{s}-1)$

c changed to $E=0.03 \mathrm{ev}$ and $\mathrm{v}=3.81 \mathrm{e} 4 \mathrm{~cm} / \mathrm{s}$ for our params (AMK 10/14/04) Eth $=0.03$

$\mathrm{vth}=3.81 \mathrm{e} 4$

! Eth $=0.0$

! vth $=0.0$

do $60 \mathrm{n}=1, \mathrm{Ntot}$

do $59 \mathrm{iz}=0, \mathrm{Nz}$

! $\quad \mathrm{iz}=0$

$\operatorname{kionth}(\mathrm{n}, \mathrm{iz})=\operatorname{Sionth}(\mathrm{n}, \operatorname{Eion}(\mathrm{n})) * \mathrm{vth} * \mathrm{xn} 0(\mathrm{iz})$

! if(iz.eq.0)write(*,*)'Sionth',n,Sionth(n,Eion(n))

$\operatorname{rrecth}(\mathrm{n}, \mathrm{iz})=\operatorname{Sreci}(\operatorname{Eth}, \mathrm{n}, \operatorname{Eion}(\mathrm{n}), \mathrm{g}(\mathrm{n}), \mathrm{gi}(\mathrm{n}))^{*} \mathrm{vth}^{*} \mathrm{xn0} 0(\mathrm{iz})^{*}$

. nes(iz)*fac(n)*ni(iz)

! $\quad$ if(iz.eq.0)write( $\left.{ }^{*},{ }^{*}\right)$ 'Sreci',n,Sreci(Eth,n,Eion(n),g(n),gi(n))

do $61 \mathrm{~m}=\mathrm{n}+1$,Ntot

$\operatorname{kexcth}(n, m, i z)=\operatorname{Sexcth}(n, m, \operatorname{Eexc}(n), \operatorname{Eexc}(m), g i(n), g i(m)) * v t h$

*xn0(iz)

if(iz.eq.0)write(*,*)'Sexcth',n,m,Sexcth(n,m,Eexc(n),Eexc(m),

! . $\quad$ gi(n),gi(m)),gi(n),gi(m),Eexc(m)-Eexc(n)

61 continue

do $62 \mathrm{l}=1, \mathrm{n}-1$

$\operatorname{kdeexth}(n, 1,1 \mathrm{iz})=\operatorname{Sdeexth}(\mathrm{n}, 1, \operatorname{Eexc}(\mathrm{n}), \operatorname{Eexc}(1), \mathrm{g}(\mathrm{n}), \mathrm{g}(1), \mathrm{gi}(\mathrm{n})$,

. $\operatorname{gi}(1), \mathrm{g} 0(\mathrm{n}))^{*} \mathrm{vth}^{*} \mathrm{xn} 0(\mathrm{iz})$

if(iz.eq.0)write( $\left.{ }^{*},{ }^{*}\right)$ 'Sdeexth',n,1,Sdeexth(n,1,Eexc(n),Eexc(1)

! . . g(n),g(l),gi(n),gi(l),g0(n)),gi(n),gi(l),Eexc(n)-Eexc(l)

62 continue

59 continue

60 continue

! stop

c calculation of the escape factors (KEEP THIS)

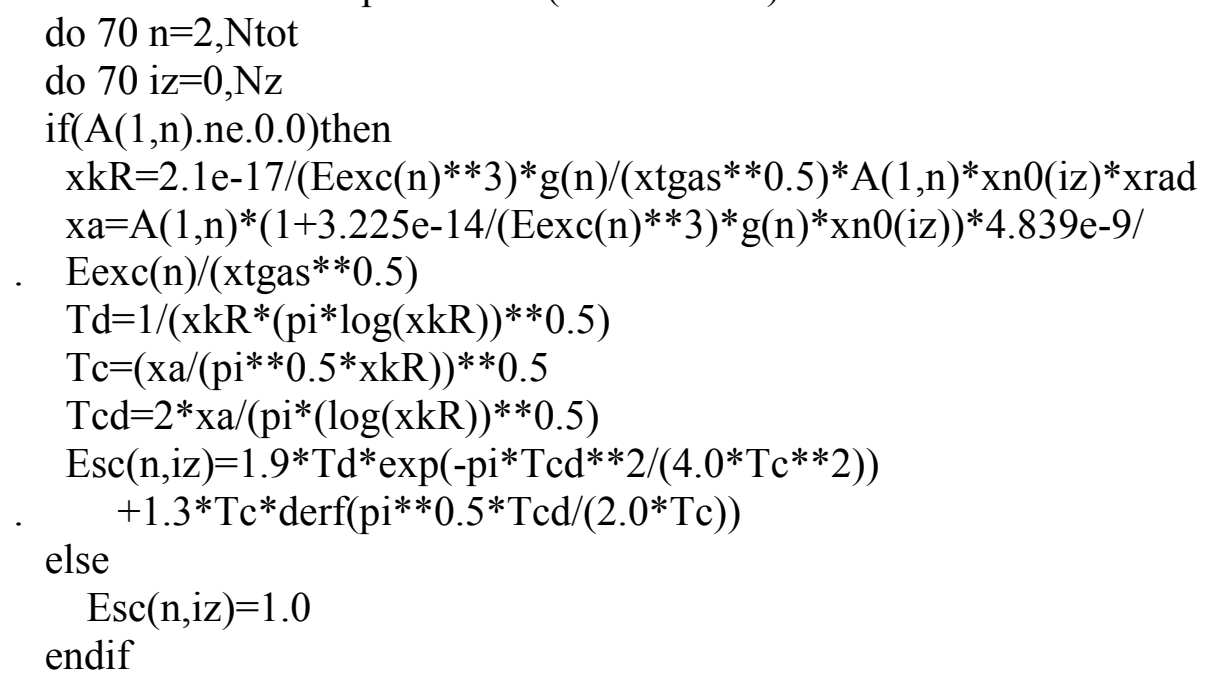




\section{c CALCULATION OF THE LEVEL POPULATIONS}

c Initial values: all populations $=0$, $\operatorname{pop}($ ground; $\mathrm{n}=1)=\mathrm{xngas}$

$$
\begin{aligned}
\text { do } 100 \mathrm{iz} & =0, \mathrm{Nz} \\
\text { pop }(1, \mathrm{iz}) & =\mathrm{xn} 0(\mathrm{iz}) \\
\text { do } 110 \mathrm{n} & =2, \mathrm{Ntot} \\
\text { pop }(\mathrm{n}, \mathrm{iz}) & =0.0 \\
110 \quad \text { continue } &
\end{aligned}
$$$$
100 \text { continue }
$$

c ct terms (indep.of time) for all levels: loss by :

do $120 \mathrm{n}=2$,Ntot

do $120 \mathrm{iz}=1, \mathrm{Nz}-1$

$\operatorname{Kloss}(\mathrm{n}, \mathrm{iz})=0.0$

$\mathrm{c}^{*}$ electron, ion, fast atom, therm.atom excitation to higher levels do $130 \mathrm{~m}=\mathrm{n}+1$,Ntot

$\operatorname{Kloss}(\mathrm{n}, \mathrm{iz})=\operatorname{Kloss}(\mathrm{n}, \mathrm{iz})+\operatorname{kexce}(\mathrm{n}, \mathrm{m}, \mathrm{iz})+\mathrm{kexci}(\mathrm{n}, \mathrm{m}, \mathrm{iz})+$

kexca(n,m,iz)+kexcth(n,m,iz)

130 continue

c* elec,ion,fast atom,therm.atom deexcit, radiat.decay to lower levels

c (escape factors to level 1 incorporated!!)

do $1401=1, n-1$

if(1.eq.1)Arad $=\mathrm{A}(1, \mathrm{n}) * \operatorname{Esc}(\mathrm{n}, \mathrm{iz})$

if(l.ne.1) $\mathrm{Arad}=\mathrm{A}(1, \mathrm{n})$

$\operatorname{Kloss}(\mathrm{n}, \mathrm{iz})=\operatorname{Kloss}(\mathrm{n}, \mathrm{iz})+\mathrm{kdeexe}(\mathrm{n}, 1, \mathrm{iz})+\mathrm{kdeexi}(\mathrm{n}, 1, \mathrm{iz})+$

. $\operatorname{kdeexa}(\mathrm{n}, 1, \mathrm{iz})+\mathrm{kdeexth}(\mathrm{n}, 1, \mathrm{iz})+$ Arad

! if(iz.eq.1)write( $\left.{ }^{*}, *\right) k d e e x e(n, 1, i z), k d e e x i(n, 1, i z)$,

! . kdeexa(n,l,iz),kdeexth(n,l,iz),Arad

140 continue

$\mathrm{c}^{*}$ electron, ion, fast atom, therm.atom ionization

$\operatorname{Kloss}(\mathrm{n}, \mathrm{iz})=\operatorname{Kloss}(\mathrm{n}, \mathrm{iz})+\operatorname{kione}(\mathrm{n}, \mathrm{iz})+\operatorname{kioni}(\mathrm{n}, \mathrm{iz})+\operatorname{kiona}(\mathrm{n}, \mathrm{iz})+$

kionth $(\mathrm{n}, \mathrm{iz})$

120 continue

c ct.terms (indep.of time) for metast: 2,4

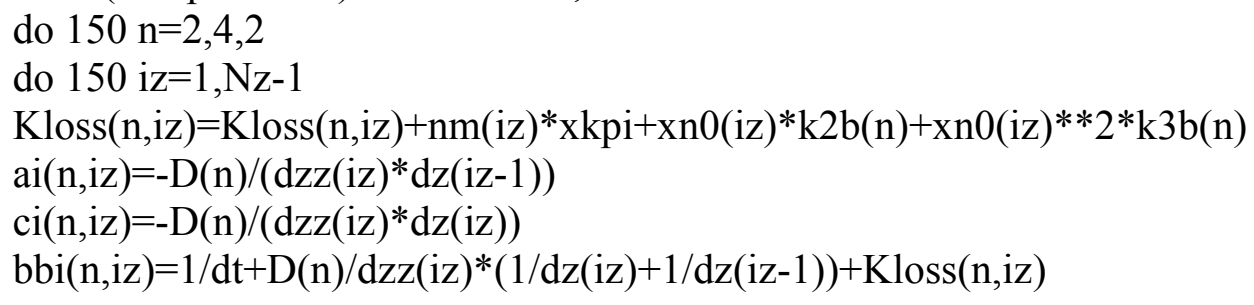


c Each timestep (pop(1->n-1) known at t, pop(n+1->Ntot) known at t-1)

$$
\text { mult }=10
$$

$\mathrm{t}=0.0$

do $200 \mathrm{it}=1,10000000$

$$
\mathrm{t}=\mathrm{t}+\mathrm{dt}
$$

c old values

$$
\text { do } 210 \mathrm{n}=2,65
$$

do $210 \mathrm{iz}=0, \mathrm{Nz}$

210

$$
\operatorname{popold}(\mathrm{n}, \mathrm{iz})=\operatorname{pop}(\mathrm{n}, \mathrm{iz})
$$

c each level

c metastable levels: 2,4

do $300 n=2,4,2$

$$
\text { do } 310 \mathrm{iz}=1, \mathrm{Nz}-1
$$

c additional loss (met-met coll; incorpor.in bi)

$$
\begin{aligned}
& \text { if(n.eq.2)pop2 }=\operatorname{pop}(n+2, i z) \\
& \text { if(n.eq.4)pop2 }=\operatorname{pop}(n-2, i z) \\
& \text { bi=bbi(n,iz)+2*xkmet*pop(n,iz)+xkmet*pop2 } \\
& \text { prod }=0.0
\end{aligned}
$$

c prod: excit.from lower levels

$$
\begin{aligned}
& \text { do } 3201=1, n-1 \\
& \operatorname{prod}=\operatorname{prod}+(\operatorname{kexce}(1, n, i z)+\operatorname{kexci}(1, n, i z)+\operatorname{kexca}(1, n, i z)+
\end{aligned}
$$

. $\operatorname{kexcth}(1, n, i z))^{*} \operatorname{pop}(1, \mathrm{iz})$

320 continue

c prod: deexcit.+rad.decay from higher levels do $330 \mathrm{~m}=\mathrm{n}+1$,Ntot $\operatorname{prod}=\operatorname{prod}+(\operatorname{kdeexe}(\mathrm{m}, \mathrm{n}, \mathrm{iz})+\mathrm{kdeexi}(\mathrm{m}, \mathrm{n}, \mathrm{iz})+\mathrm{kdeexa}(\mathrm{m}, \mathrm{n}, \mathrm{iz})+$

. $\operatorname{kdeexth}(m, n, i z)+A(n, m)) * p o p(m, i z)$

330 continue

c prod: recomb -> total prod

$\operatorname{prod}=\operatorname{prod}+\operatorname{rrec} 1 \mathrm{e}(\mathrm{n}, \mathrm{iz})+\operatorname{rrec} 2 \mathrm{e}(\mathrm{n}, \mathrm{iz})+\operatorname{rreci}(\mathrm{n}, \mathrm{iz})+\operatorname{rreca}(\mathrm{n}, \mathrm{iz})+$

- $\operatorname{rrecth}(n, i z)$

$\mathrm{di}=\operatorname{popold}(\mathrm{n}, \mathrm{iz}) / \mathrm{dt}+\operatorname{prod}$

c Thomas algorithm

if(iz.eq. 1)beta(iz) $=$ bi

if(iz.ne.1)beta(iz)=bi-ai(n,iz)*ci(n,iz-1)/beta(iz-1)

if(iz.eq.1)gamma(iz) $=$ di

if(iz.ne.1)gamma(iz)=di-ai(n,iz)* gamma(iz-1)/beta(iz-1)

310 continue 


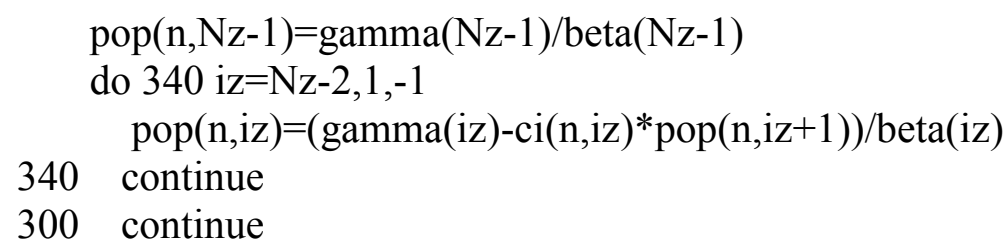


c new timestep: calculate deviation

$$
\mathrm{xmaxdev}=0.0
$$

do $500 \mathrm{n}=2,65$

do $500 \mathrm{iz}=1, \mathrm{Nz}-1$

if((pop(n,iz).ne.0.0).or.(popold(n,iz).ne.0.0))

- $\quad \operatorname{dev}=\operatorname{abs}(\operatorname{pop}(n, i z)-\operatorname{popold}(n, i z)) * 2 /(\operatorname{pop}(n, i z)+\operatorname{popold}(n, i z))$

500 continue

if(it.eq.mult)then

mult $=$ mult +10

endif

if(xmaxdev.lt.1.e-6)goto 111

! if(it.eq.1)goto 111

200 continue

111 write( $\left(*,{ }^{*}\right)$ 't is',t,xmaxdev

c OUTPUT

c level populations

do $612 \mathrm{n}=1$,Ntot

$\mathrm{n} 2(\mathrm{n})=\mathrm{n}$

612 continue

write $(14,611) \mathrm{z}(0),(\mathrm{n} 2(\mathrm{n}), \mathrm{n}=1, \mathrm{~N}$ tot $)$

do $600 \mathrm{iz}=0, \mathrm{Nz}$

write $(14,610) r(i z),(\operatorname{pop}(n, i z), n=1, N t o t)$

! write $(14,610) z(\mathrm{iz}),(\operatorname{pop}(\mathrm{n}, \mathrm{iz}), \mathrm{n}=1, \mathrm{Ntot})$

600 continue

610 format(f8.5,65(',',e10.5))

611 format(f7.5,65(',',i9))

stop

c contributions of production, loss processes

write $\left(15,{ }^{*}\right)$ 'n: prod: rec1e,rec2e,reci,reca,recth'

write $\left(15,{ }^{*}\right)^{\prime}$ e: exc(from 1$)$, deexc(from m $)^{\prime}$

write $\left(15,{ }^{*}\right)^{\prime} \mathrm{i}$ : exc(from 1), deexc(from m)'

write $\left(15,{ }^{*}\right)^{\prime}$ a: exc(from 1), deexc(from m)'

write $\left(15,{ }^{*}\right)^{\prime}$ th: exc(from 1),deexc(from m)'

write $\left(15,{ }^{*}\right)$ 'rad.decay (from $\mathrm{m}$ )'

write $\left(15,{ }^{*}\right)$ 'n: loss: ione,ioni,iona,ionth'

write $\left(15,{ }^{*}\right)^{\prime} \mathrm{e}: \operatorname{exc}($ to $\mathrm{m}), \operatorname{deexc}(\text { to } 1)^{\prime}$

write $\left(15,{ }^{*}\right)^{\prime} \mathrm{i}$ : exc(to $\left.\mathrm{m}\right), \operatorname{deexc}(\text { to } 1)^{\prime}$

write $\left(15,{ }^{*}\right)^{\prime}$ a: $\operatorname{exc}($ to $\mathrm{m})$, deexc(to 1$)^{\prime}$

write( $\left.15,{ }^{*}\right)^{\prime}$ th: exc(to $\left.\mathrm{m}\right), \operatorname{deexc}(\text { to } 1)^{\prime}$ 
write $(15, *)$ 'rad.decay (to 1$)^{\prime}$

write $\left(15,{ }^{*}\right)$ 'for metast: pi,2b,3b,met-met (with same, other)'

c calculate contributions

do $620 \mathrm{n}=2$,Ntot

trec $1 \mathrm{e}=0.0$

$\operatorname{trec} 2 \mathrm{e}=0.0$

treci $=0.0$

treca $=0.0$

trecth $=0.0$

tione $=0.0$

tioni $=0.0$

tiona $=0.0$

tionth $=0.0$

tpi $=0.0$

$\mathrm{t} 2 \mathrm{~b}=0.0$

$\mathrm{t} 3 \mathrm{~b}=0.0$

tmet $1=0.0$

tmet $2=0.0$

do $621 \mathrm{~m}=1$,Ntot

texce $(\mathrm{m})=0.0$

texci $(\mathrm{m})=0.0$

texca $(\mathrm{m})=0.0$

$\operatorname{texcth}(\mathrm{m})=0.0$

$\operatorname{tdeexe}(\mathrm{m})=0.0$

$\operatorname{tdeexi}(\mathrm{m})=0.0$

$\operatorname{tdeexa}(\mathrm{m})=0.0$

$\operatorname{tdeexth}(\mathrm{m})=0.0$

$\operatorname{trad}(\mathrm{m})=0.0$

621 continue

c sum over all positions

do $630 \mathrm{iz}=1, \mathrm{Nz}-1$

c recomb(prod), ioniz(loss)

trecle $=$ trecle+rrecle $(\mathrm{n}, \mathrm{iz}) * \mathrm{dzz}(\mathrm{iz})$

trec $2 \mathrm{e}=\operatorname{trec} 2 \mathrm{e}+\operatorname{rrec} 2 \mathrm{e}(\mathrm{n}, \mathrm{iz}) * \mathrm{dzz}(\mathrm{iz})$

treci $=$ treci $+\operatorname{rreci}(\mathrm{n}, \mathrm{iz})^{*} \mathrm{dzz}(\mathrm{iz})$

treca $=$ treca $+\operatorname{rreca}(\mathrm{n}, \mathrm{iz}) * \mathrm{dzz}(\mathrm{iz})$

trecth $=\operatorname{trecth}+\operatorname{rrecth}(\mathrm{n}, \mathrm{iz}) * \mathrm{dzz}(\mathrm{iz})$

tione $=$ tione + kione $(\mathrm{n}, \mathrm{iz}) * \mathrm{dzz}(\mathrm{iz}){ }^{*} \operatorname{pop}(\mathrm{n}, \mathrm{iz})$

tioni=tioni+kioni(n,iz)*dzz(iz)*pop(n,iz)

tiona $=$ tiona + kiona $(\mathrm{n}, \mathrm{iz}) * \mathrm{dzz}(\mathrm{iz}){ }^{*}$ pop(n,iz)

tionth=tionth+kionth(n,iz)*dzz(iz)*pop(n,iz)

$\mathrm{c}$ for metastables: PI,2b,3b,met-met : addit.loss

if((n.eq.2).or.(n.eq.4))then

tpi=tpi+xkpi*nm(iz)*pop(n,iz)*dzz(iz)

$\mathrm{t} 2 \mathrm{~b}=\mathrm{t} 2 \mathrm{~b}+\mathrm{k} 2 \mathrm{~b}(\mathrm{n}) * \mathrm{xn} 0(\mathrm{iz})^{*}$ pop(n,iz)*dzz(iz) 


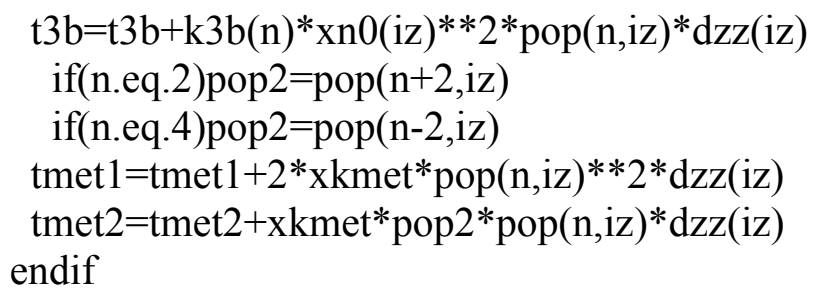


c calculation of contributions for each process

if(tprod.ne.0.)then

trec $1 \mathrm{e}=$ trec $1 \mathrm{e} / \mathrm{tprod}^{*} 100$

trec $2 \mathrm{e}=$ trec $2 \mathrm{e} /$ tprod $^{*} 100$

treci $=$ treci/tprod $* 100$

treca $=$ treca/tprod $* 100$

trecth $=$ trecth/tprod*100

tione $=$ tione $/$ tloss $* 100$

tioni $=$ tioni $/$ tloss $* 100$

tiona $=$ tiona/tloss $* 100$

tionth $=$ tionth/tloss $* 100$

if((n.eq.2).or.(n.eq.4))then

tpi $=$ tpi/tloss $* 100$

$\mathrm{t} 2 \mathrm{~b}=\mathrm{t} 2 \mathrm{~b} / \mathrm{t}$ loss $* 100$

$\mathrm{t} 3 \mathrm{~b}=\mathrm{t} 3 \mathrm{~b} / \mathrm{tloss} * 100$

tmet $1=$ tmet $1 /$ tloss $* 100$

tmet $2=$ tmet $2 /$ tloss $* 100$

endif

do $680 \mathrm{~m}=\mathrm{n}+1$,Ntot

$\operatorname{tdeexe}(\mathrm{m})=\operatorname{tdeexe}(\mathrm{m}) / \mathrm{tprod} * 100$

tdeexi $(\mathrm{m})=\operatorname{tdeexi}(\mathrm{m}) /$ tprod $^{*} 100$

$\operatorname{tdeexa}(\mathrm{m})=\operatorname{tdeexa}(\mathrm{m}) / \operatorname{tprod} * 100$

$\operatorname{tdeexth}(\mathrm{m})=\operatorname{tdeexth}(\mathrm{m}) / \operatorname{tprod} * 100$

$\operatorname{trad}(\mathrm{m})=\operatorname{trad}(\mathrm{m}) / \operatorname{tprod} * 100$

texce $(\mathrm{m})=\operatorname{texce}(\mathrm{m}) /$ tloss $* 100$

texci $(\mathrm{m})=\operatorname{texci}(\mathrm{m}) /$ tloss $* 100$

texca $(\mathrm{m})=\operatorname{texca}(\mathrm{m}) /$ tloss $* 100$

$\operatorname{texcth}(\mathrm{m})=\operatorname{texcth}(\mathrm{m}) / \operatorname{tloss} * 100$

680 continue

do $690 \mathrm{l}=1, \mathrm{n}-1$

texce $(1)=$ texce $(1) /$ tprod $* 100$

texci $(1)=$ texci $(1) /$ tprod $^{*} 100$

texca $(1)=\operatorname{texca}(1) / \operatorname{tprod} * 100$

$\operatorname{texcth}(1)=\operatorname{texcth}(1) / \operatorname{tprod} * 100$

tdeexe $(1)=$ tdeexe $(1) /$ tloss $* 100$

$\operatorname{tdeexi}(1)=\operatorname{tdeexi}(1) /$ tloss $* 100$

$\operatorname{tdeexa}(1)=\operatorname{tdeexa}(1) / \operatorname{tloss} * 100$

$\operatorname{tdeexth}(1)=\operatorname{tdeexth}(1) / \operatorname{tloss}^{*} 100$

$\operatorname{trad}(1)=\operatorname{trad}(1) / \operatorname{tloss}^{*} 100$

690 continue

c final output: contributions in \%

write $\left(15,{ }^{*}\right) ' n '$, n

write $(15, *)$ 'prod'

write $\left(15,{ }^{*}\right)$ trec1e,trec2e,treci,treca,trecth

write $\left(15,{ }^{*}\right)$

write $(15,700)(\operatorname{texce}(1), 1=1, n-1),(\operatorname{tdeexe}(m), m=n+1, N$ tot $)$ 


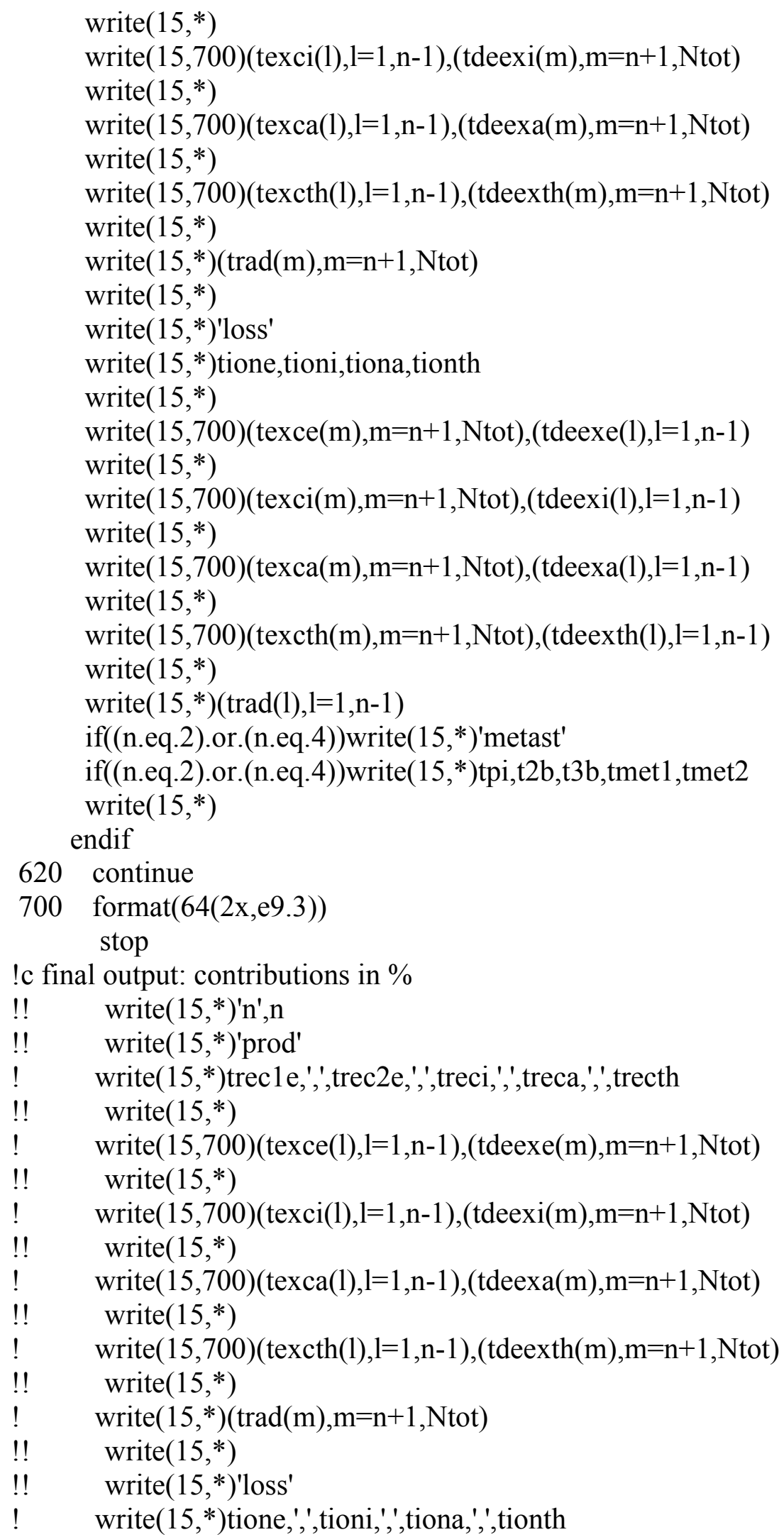




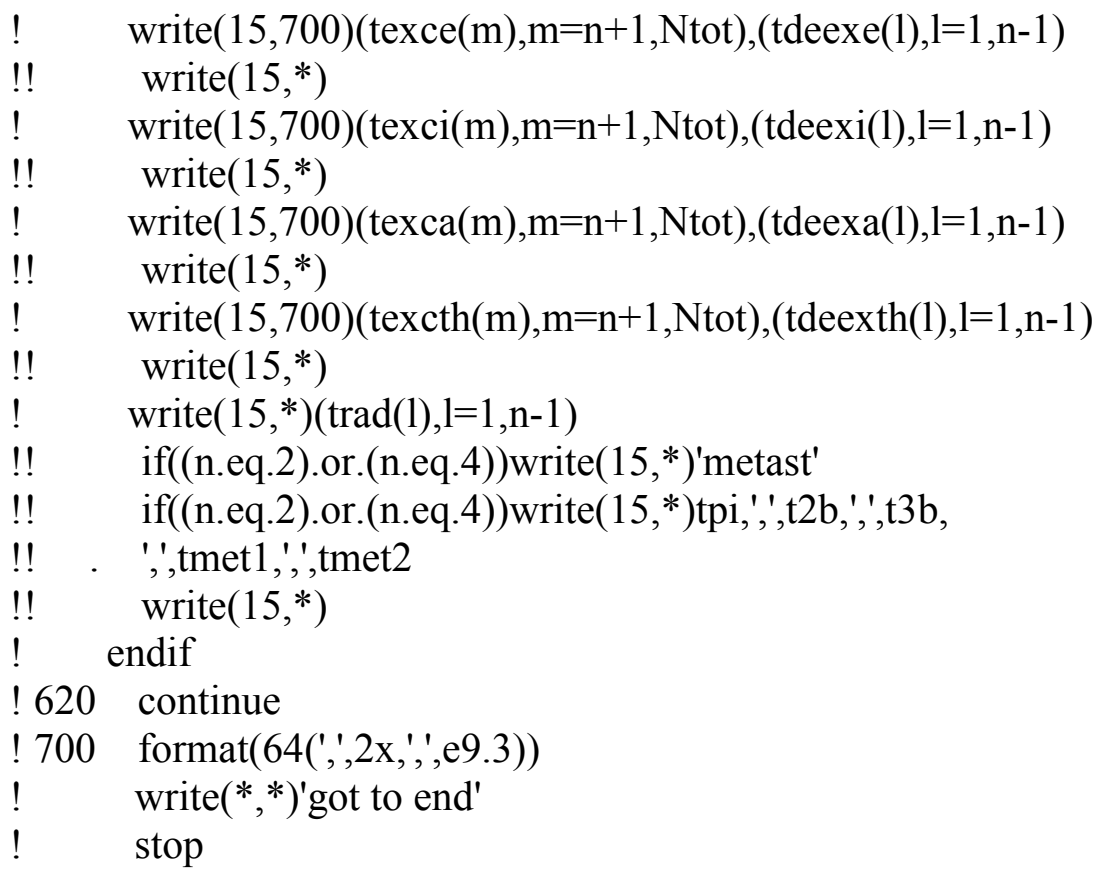

c calculation of relative relative roles of electron, ion, atom collisions

c electrons

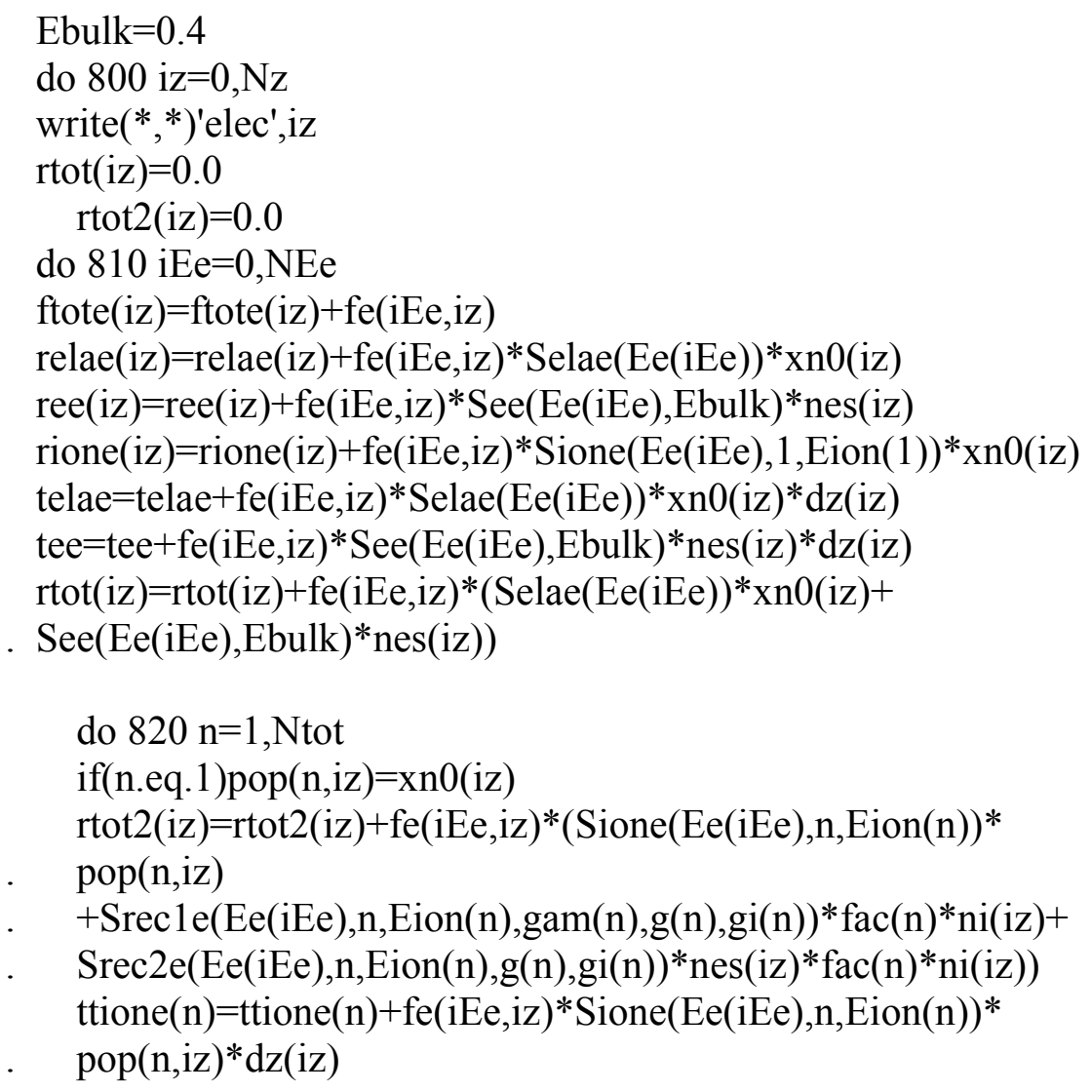




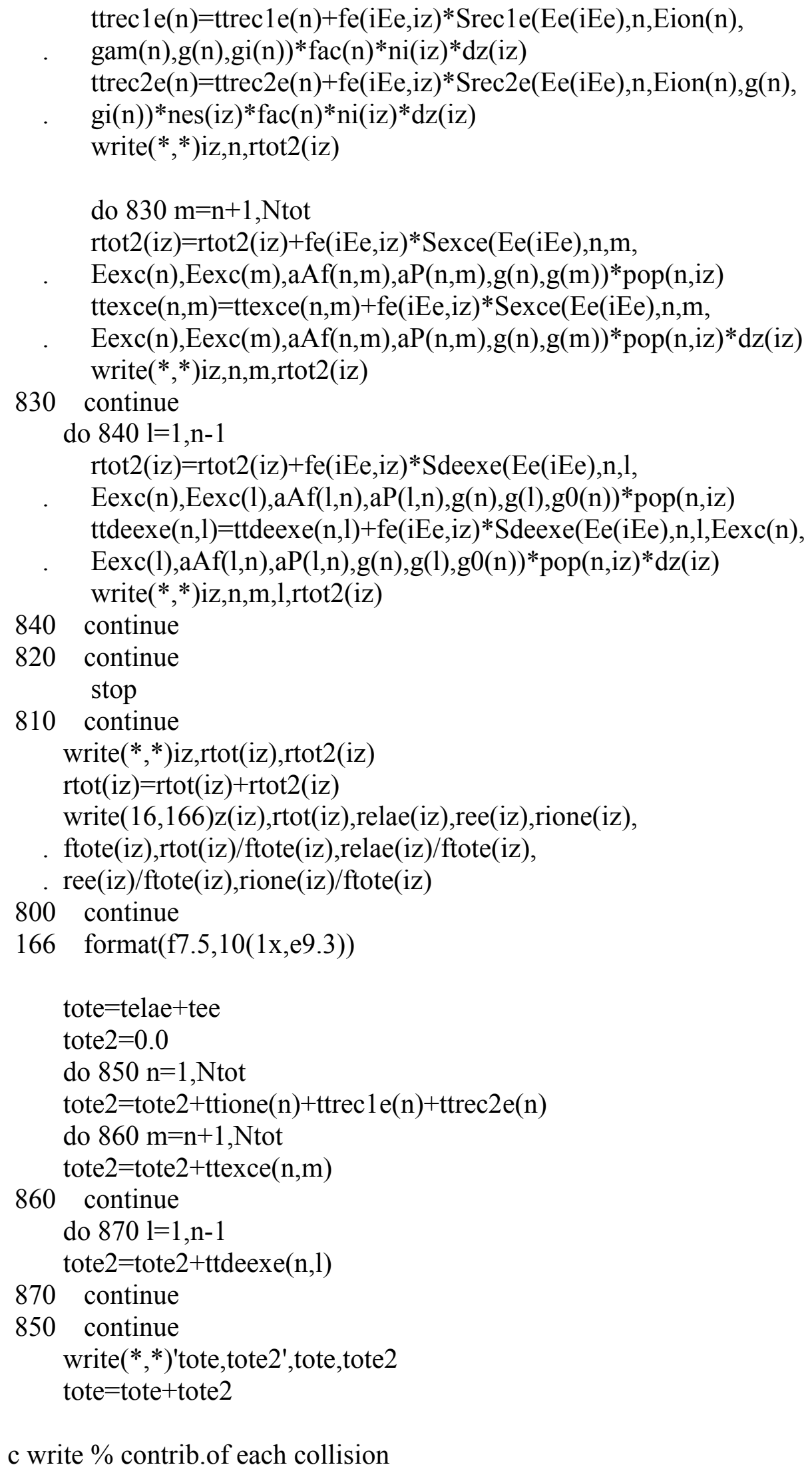

c write $\%$ contrib.of each collision 


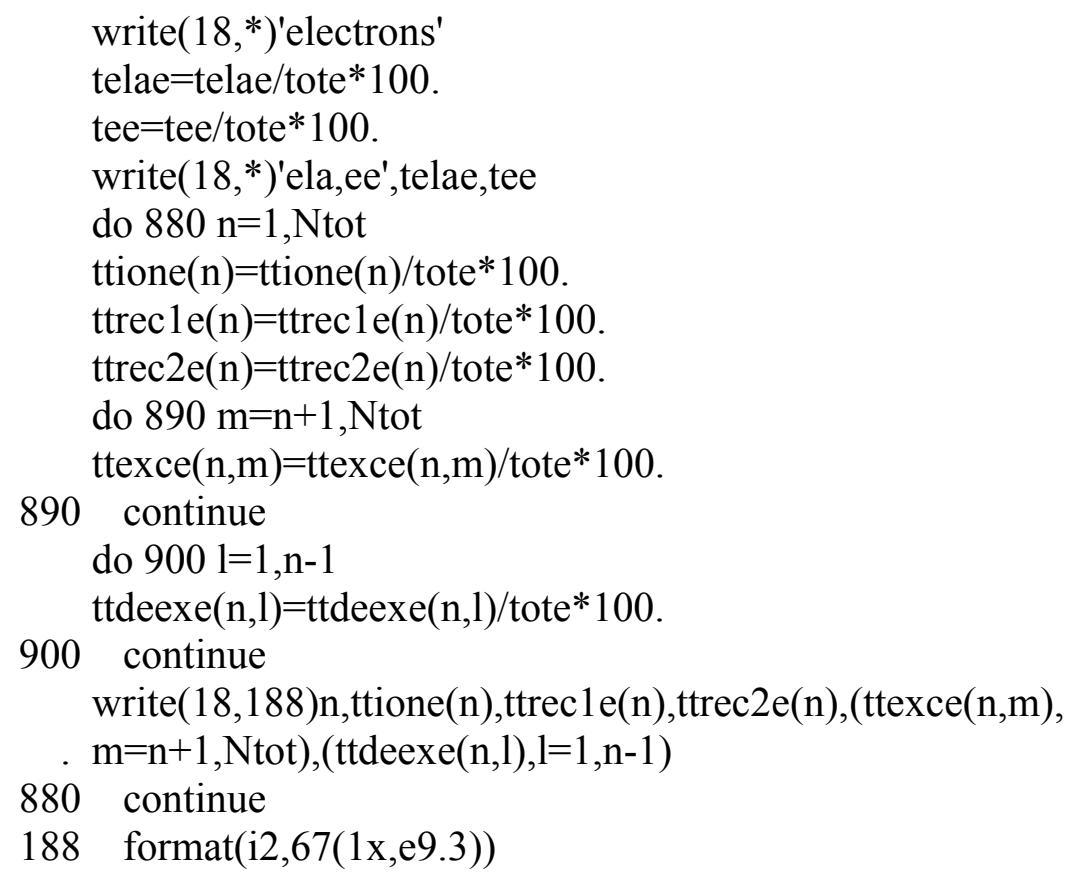

c write $\%$ contrib.of each collision write $\left(18,{ }^{*}\right)$ 'ions' tlad=tlad/toti $* 100$. telai $=$ telai $/$ toti $* 100$. telaa $=$ telaa/tota* 100 . write $\left(18,{ }^{*}\right)$ 'lad,ela',tlad,telai do $971 \mathrm{n}=1$,Ntot $\operatorname{ttioni}(\mathrm{n})=\operatorname{ttioni}(\mathrm{n}) / \operatorname{tot}^{*} 100$. $\operatorname{ttreci}(\mathrm{n})=\operatorname{ttreci}(\mathrm{n}) /$ toti $^{*} 100$. 


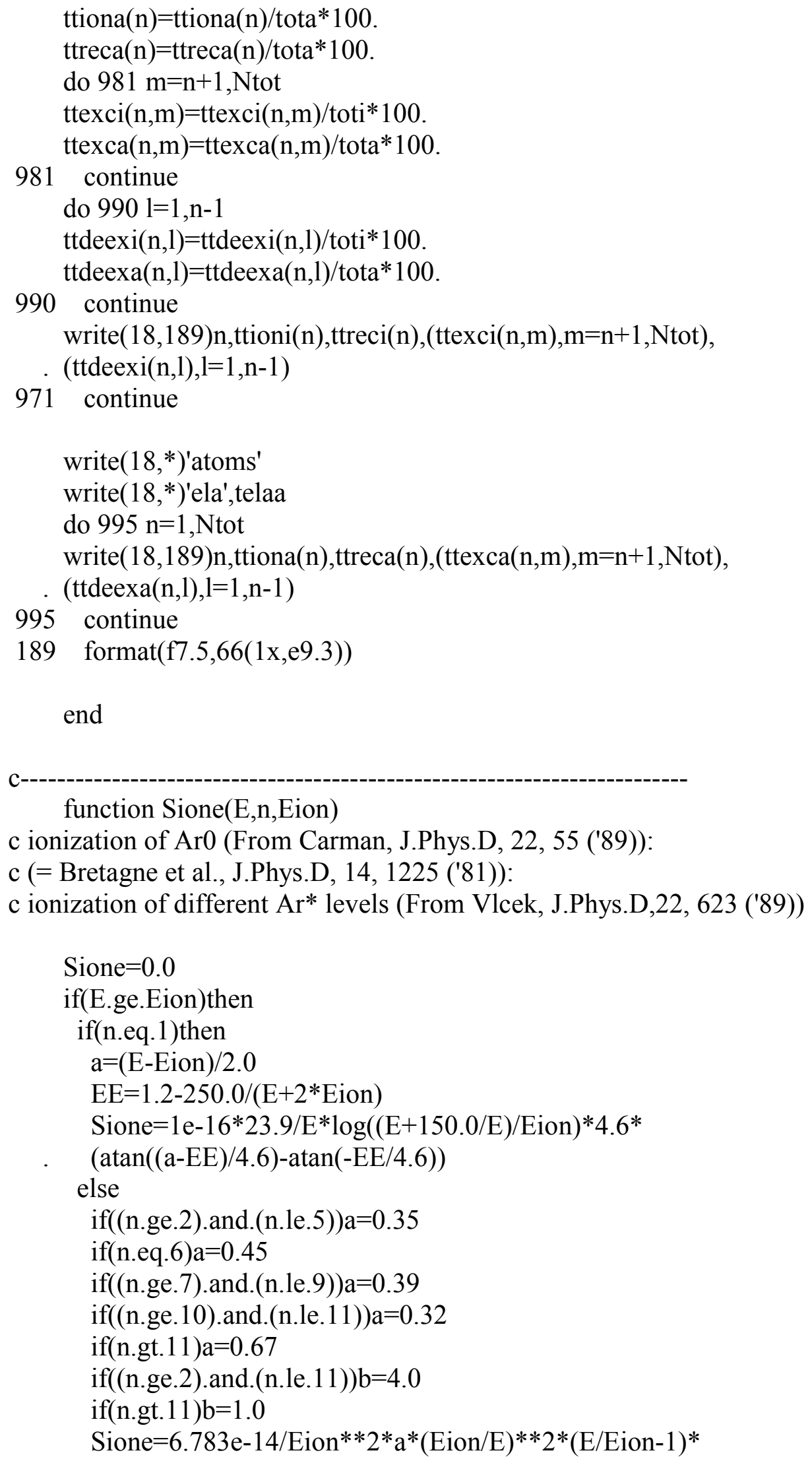

end

function Sione(E,n,Eion)

c ionization of Ar0 (From Carman, J.Phys.D, 22, 55 ('89)):

c (= Bretagne et al., J.Phys.D, 14, 1225 ('81)):

c ionization of different Ar* levels (From Vlcek, J.Phys.D,22, 623 ('89)) 


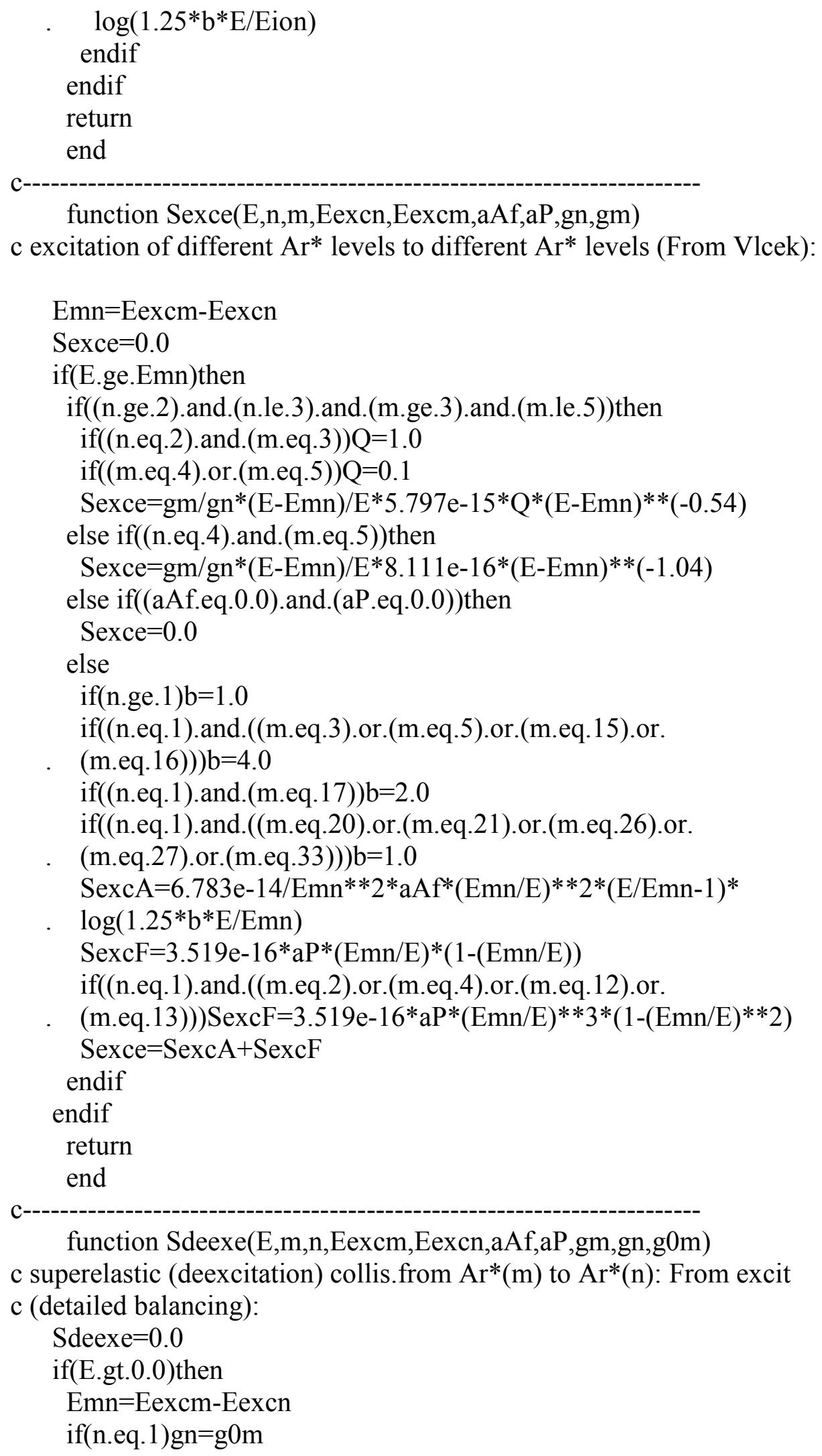




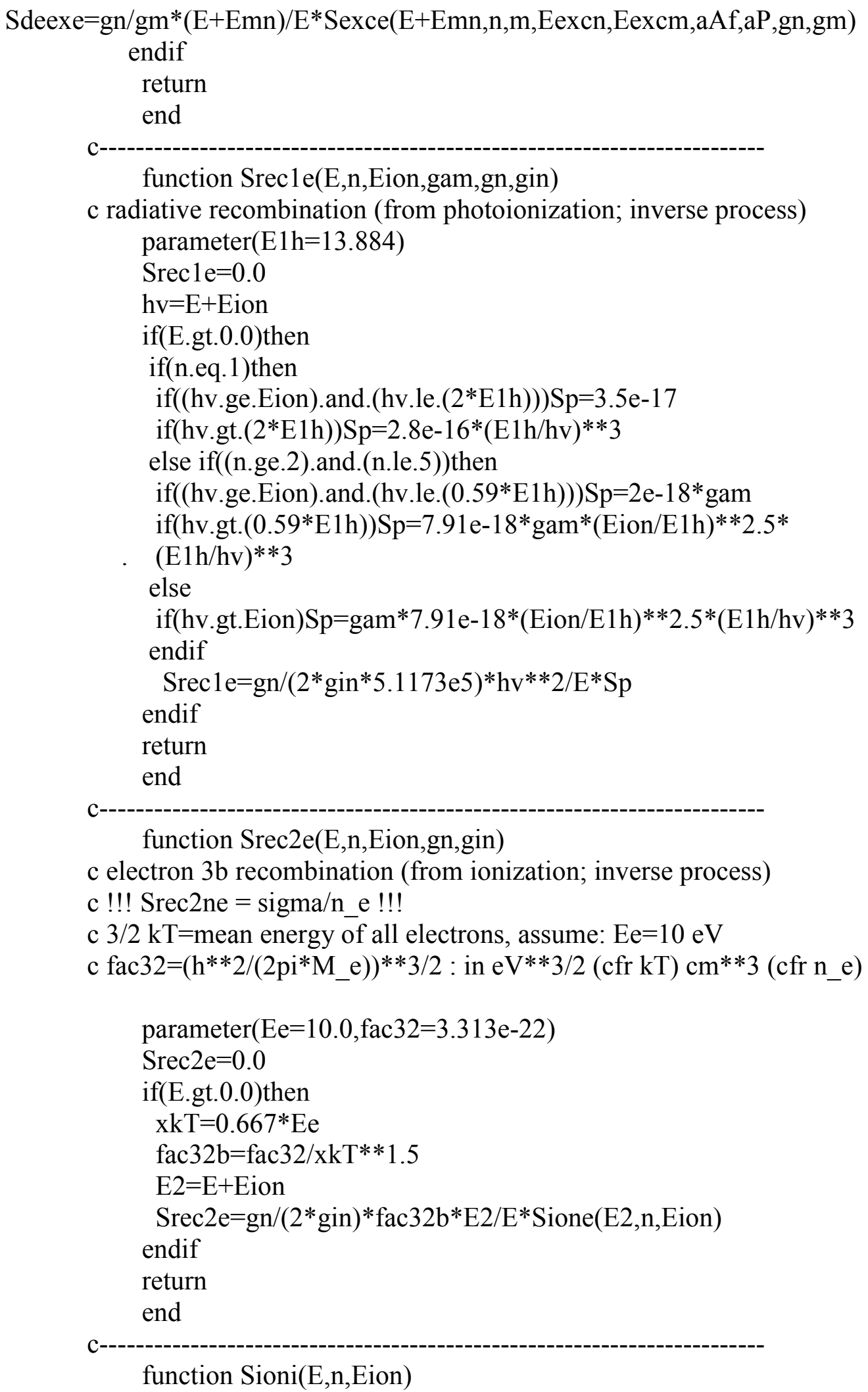




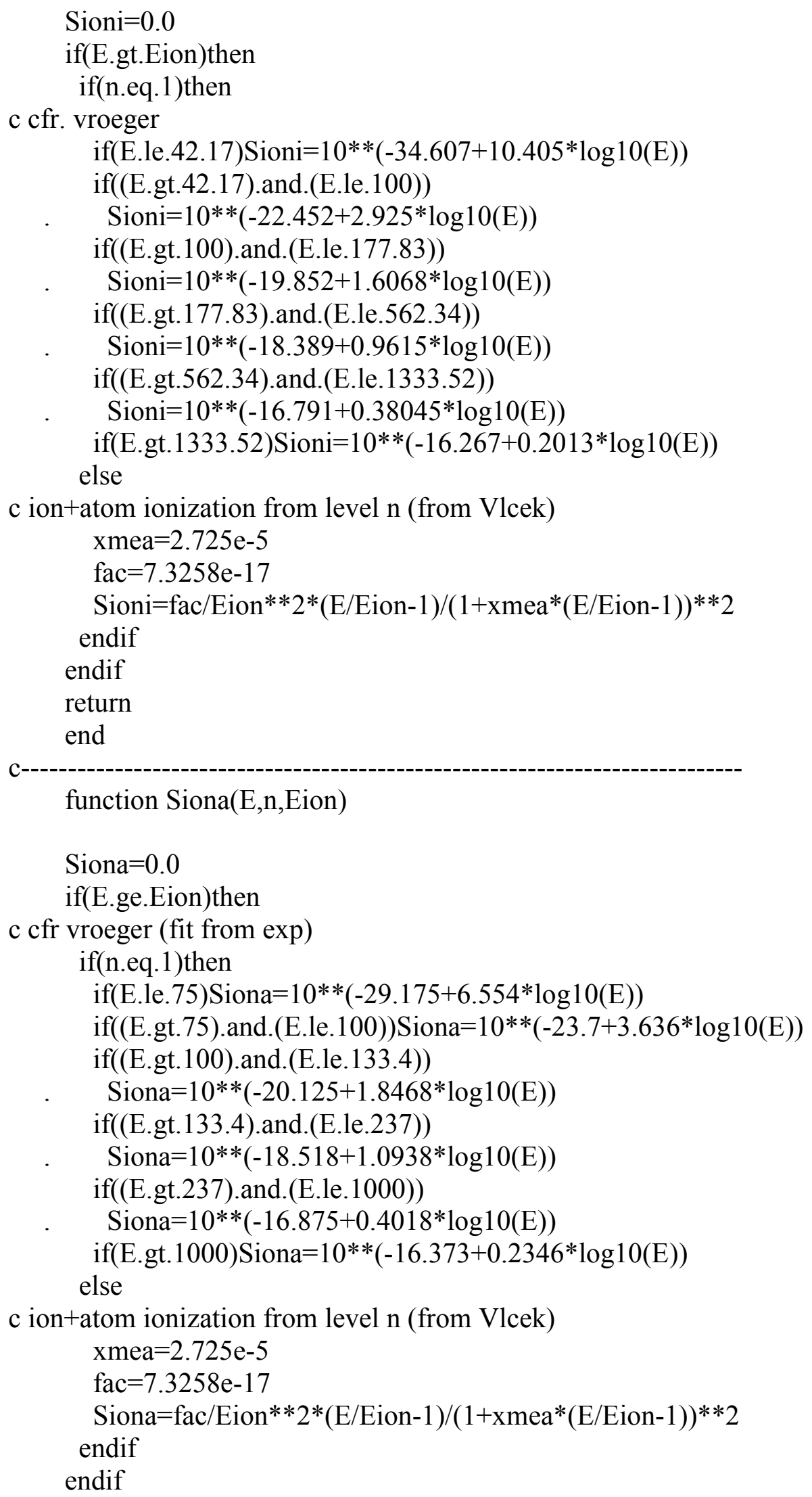




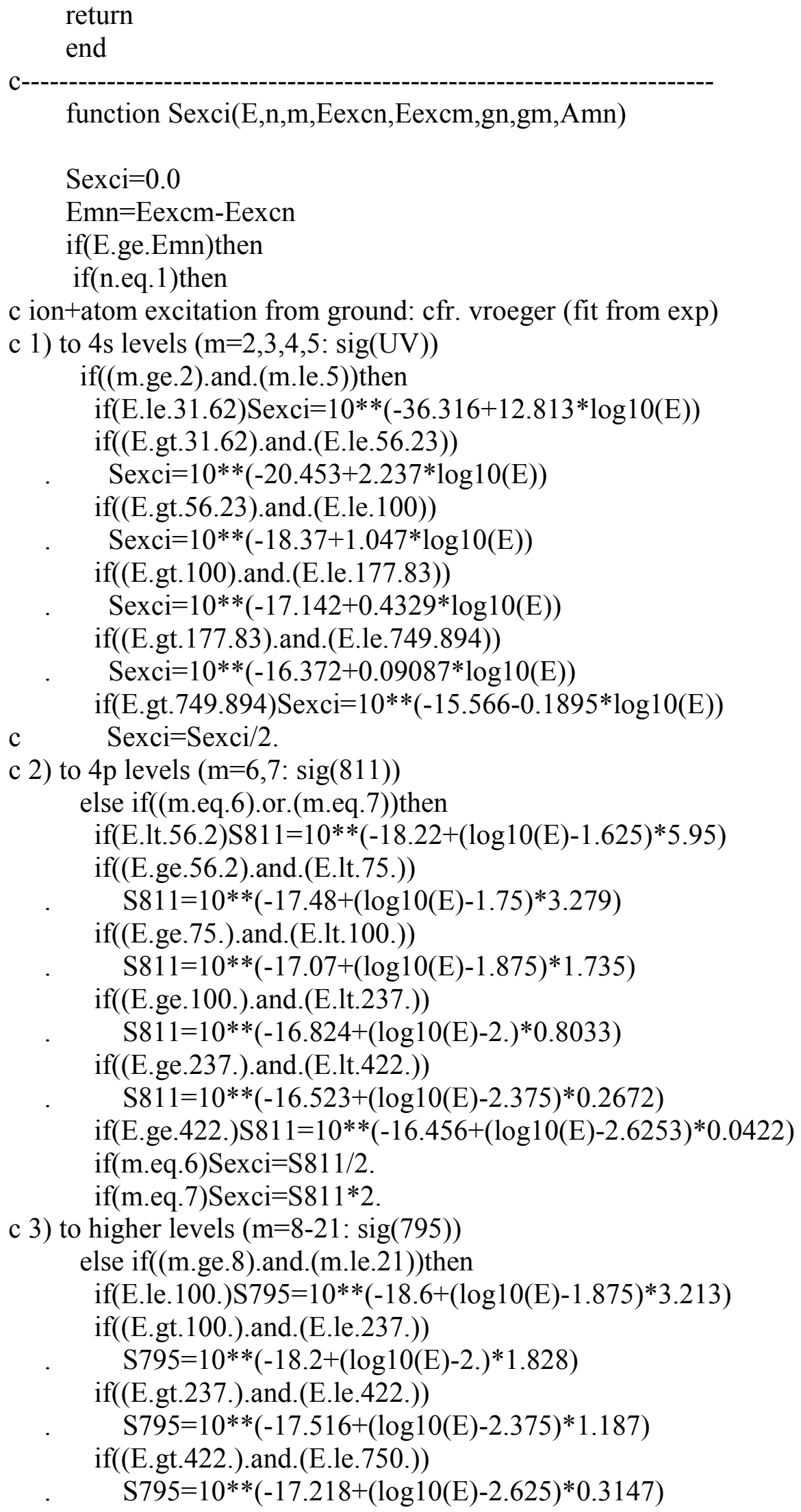


if(E.gt. 750.$) S 795=10 * *(-17.14+(\log 10(\mathrm{E})-2.875) * 0.09196)$

$\mathrm{c}$ to $4 \mathrm{p}(\mathrm{m}=8-11)$

if $($ m.eq. 8$)$ Sexci $=\mathrm{S} 795 * 2$.

if((m.eq.9).or.(m.eq.10))Sexci=S795

if(m.eq.11)Sexci $=$ S795/3.

$\mathrm{c}$ to $3 \mathrm{~d}, 5 \mathrm{~s}(\mathrm{~m}=12-17)$

if((m.ge.12).and.(m.le.17))Sexci=S795/3.

$\mathrm{c}$ to $5 \mathrm{p}(\mathrm{m}=18,19)$

if(m.eq. 18)Sexci $=\mathrm{S} 795 * 0.261$

if(m.eq. 19)Sexci $=\mathrm{S} 795 * 0.174$

$\mathrm{c}$ to $4 \mathrm{~d}, 6 \mathrm{~s}(\mathrm{~m}=20,21)$

if(m.eq.20)Sexci $=\mathrm{S} 795 / 2$.

if(m.eq.21)Sexci=S795/4.

else if(m.gt.21)then

Sexci $=0.0$

endif

else

$\mathrm{c}$ ion+atom excitation from level $\mathrm{n}$ to level $\mathrm{m}$ (from Vlcek)

$\mathrm{xmea}=2.725 \mathrm{e}-5$

$\mathrm{fac}=7.3258 \mathrm{e}-17$

$\mathrm{fmn}=\mathrm{gm} / \mathrm{gn} * 2.3073 \mathrm{e}-8 * \mathrm{Amn} / \mathrm{Emn} * * 2$

Sexci $=$ fac/Emn** $2 *$ fmn*(E/Emn-1)/(1+xmea*(E/Emn-1) $) * 2$

endif

endif

return

end

function Sdeexi(E,m,n,Eexcm,Eexcn,gm,gn,g0m,Amn)

$\mathrm{c}$ ion+atom deexcitation from level $\mathrm{m}$ to level $\mathrm{n}$ (from excitation)

Sdeexi $=0.0$

if(E.gt.0.0)then

$\mathrm{Emn}=$ Eexcm-Eexcn

if(n.eq.1)gn $=\mathrm{g} 0 \mathrm{~m}$

Sdeexi=gn/gm*(E+Emn)/E*Sexci(E+Emn,n,m,Eexcn,Eexcm,gn,gm,Amn) endif

return

end

function Sreci(E,n,Eion,gn,gin)

$\mathrm{c}$ ion + atom recomb to level $\mathrm{n}$ (from ionization:Siona)

$\mathrm{c}$ will be not used in MC

c !!! Srec $=$ sigma $/$ ne

parameter $(\mathrm{Ee}=10.0, \mathrm{fac} 32=3.313 \mathrm{e}-22)$

Sreci $=0.0$

if(E.gt.0.0)then

$\mathrm{xkT}=0.667 * \mathrm{Ee}$ 


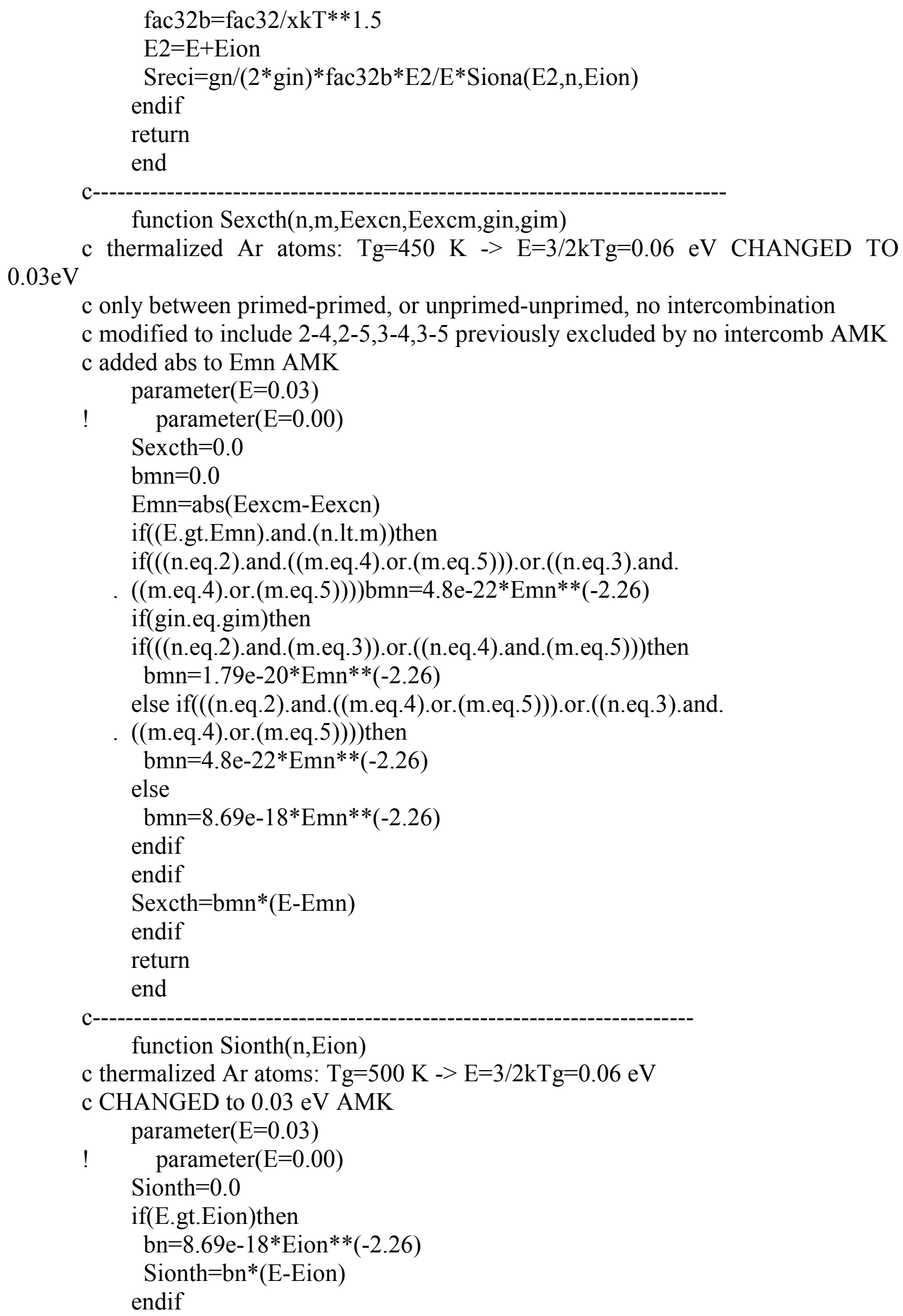




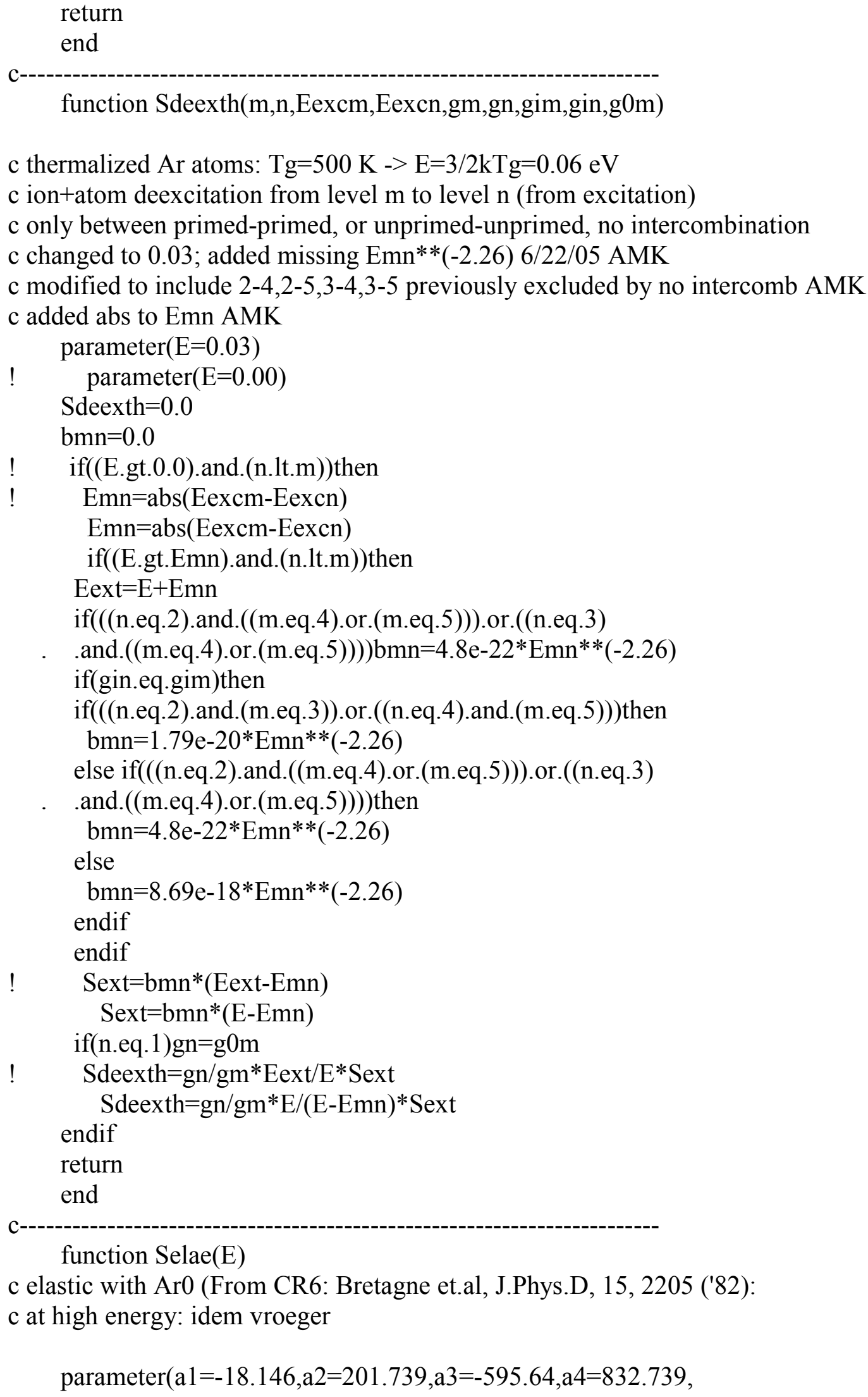


. $\mathrm{a} 5=-611.768, \mathrm{a} 6=230.268, \mathrm{a} 7=-35.192)$

Selae $=0.0$

if $(($ E.gt.0.0).and.(E.1t.4. $))$ Selae $=((\mathrm{E} /(\mathrm{E}+0.3)) * * 0.5 * \log (\mathrm{E}+0.3) *$

. $(\mathrm{a} 1+\mathrm{a} 2 /(\mathrm{E}+0.3) * * 0.5+\mathrm{a} 3 /(\mathrm{E}+0.3)+\mathrm{a} 4 /(\mathrm{E}+0.3) * * 1.5+\mathrm{a} 5 /(\mathrm{E}+0.3) * * 2+$

. $\mathrm{a} 6 /(\mathrm{E}+0.3) * * 2.5+\mathrm{a} 7 /(\mathrm{E}+0.3) * * 3)-1.319 * \log (\mathrm{E})+0.231) * 1 \mathrm{e}-16$ if((E.ge.4.).and.(E.le.16))Selae $=1.56798 \mathrm{e}-16+5.96811 \mathrm{e}-17 * \mathrm{E}+$

. $2.95251 \mathrm{e}-17 * \mathrm{E} * * 2-1.98062 \mathrm{e}-18 * \mathrm{E} * * 3+2.67756 \mathrm{e}-20 * \mathrm{E} * * 4$ if((E.gt.16).and.(E.1e.100))Selae $=4.65827 \mathrm{e}-15-2.07313 \mathrm{e}-16^{*} \mathrm{E}$

. $+4.50635 \mathrm{e}-18 * \mathrm{E}^{* *} 2-4.97379 \mathrm{e}-20 * \mathrm{E}^{* *} 3+2.68443 \mathrm{e}-22 * \mathrm{E}^{* * 4}-$

. $5.61251 \mathrm{e}-25 * \mathrm{E} * * 5$

if(E.gt.100)Selae $=5.90086 \mathrm{e}-15 * E^{* *}(-0.544327)$

return

end

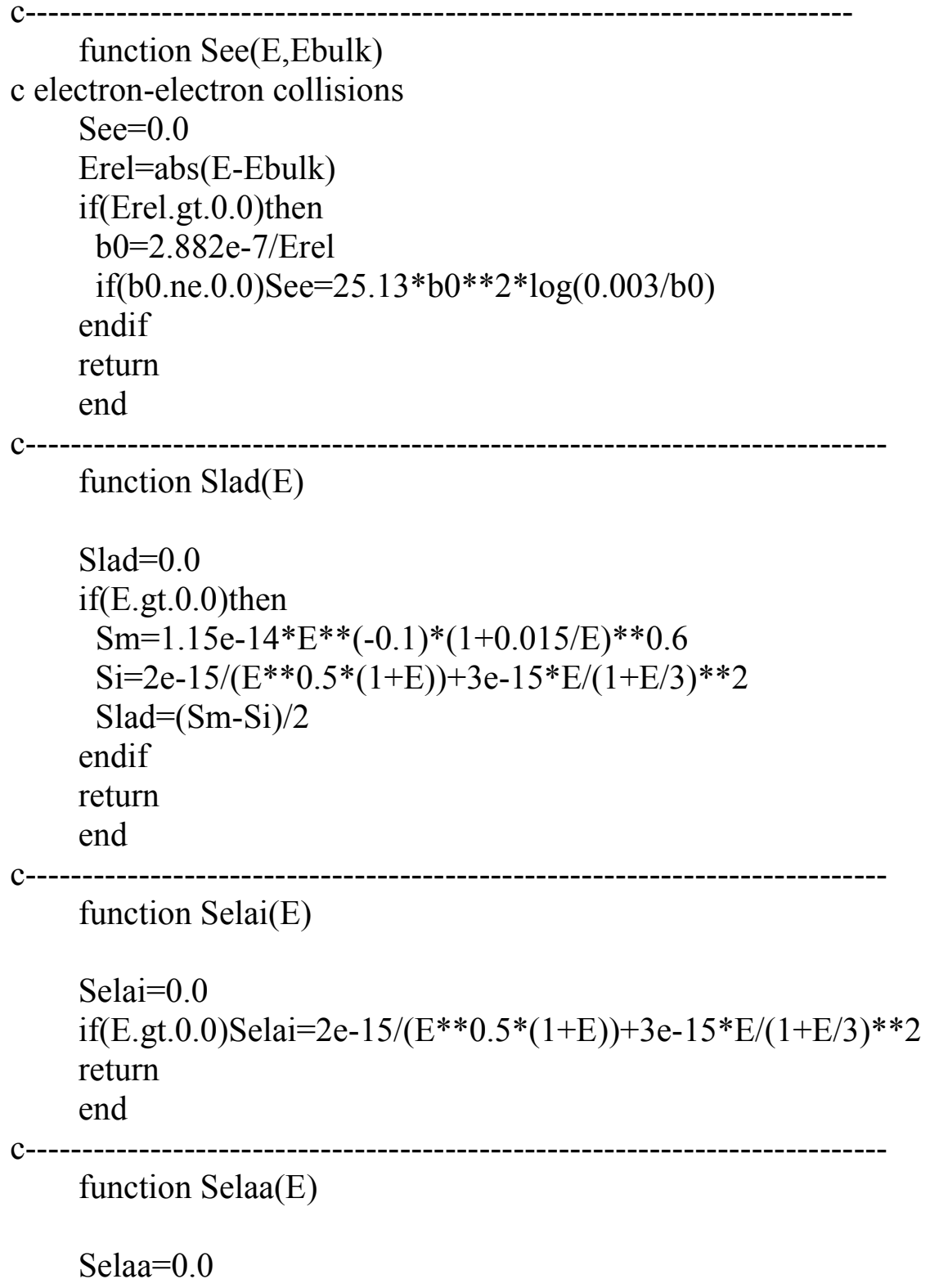

Selaa $=0.0$ 
if(E.gt.0.0)Selaa $=1.22 \mathrm{e}-15 * \mathrm{E}^{* *}(-0.5) *(1+\mathrm{E}) * * 0.24$

return

end

c error function in code did not compile, added 9/22/03 by AMK function $\operatorname{derf}(\mathrm{E})$

$\operatorname{der} f=0.0$

if (E.1t.0.)then

$\operatorname{derf}=-\operatorname{gammp}\left(.5, \mathrm{E}^{* * 2}\right)$

else

endif

$\operatorname{derf}=\operatorname{gammp}\left(.5, \mathrm{E}^{* *} 2\right)$

return

end

c gamma function added for error function 9/22/03 AMK

FUNCTION gammp $(\mathrm{a}, \mathrm{x})$

REAL a,gammp,x

c USES gcf,gser

REAL gammcf,gamser,gln

if(x.1t.0..or.a.le.0.)pause

if(x.lt.a+1.)then

call gser(gamser,a,x,gln)

gammp=gamser

else

call gcf(gammcf,a,x,gln)

endif

gammp=1.-gammcf

return

END

c gamma function added for error function 9/22/03 AMK

SUBROUTINE gser(gamser,a,x,gln)

INTEGER ITMAX

REAL a,gamser,gln, $x, E P S$

PARAMETER (ITMAX=100,EPS=3.e-7)

c USES gammln

INTEGER $\mathrm{n}$

REAL ap,del,sum,gammln

$\operatorname{gln}=\operatorname{gamm} \ln (\mathrm{a})$

if(x.le.0.)then

if(x.1t.0.)pause

gamser $=0$.

return

endif 


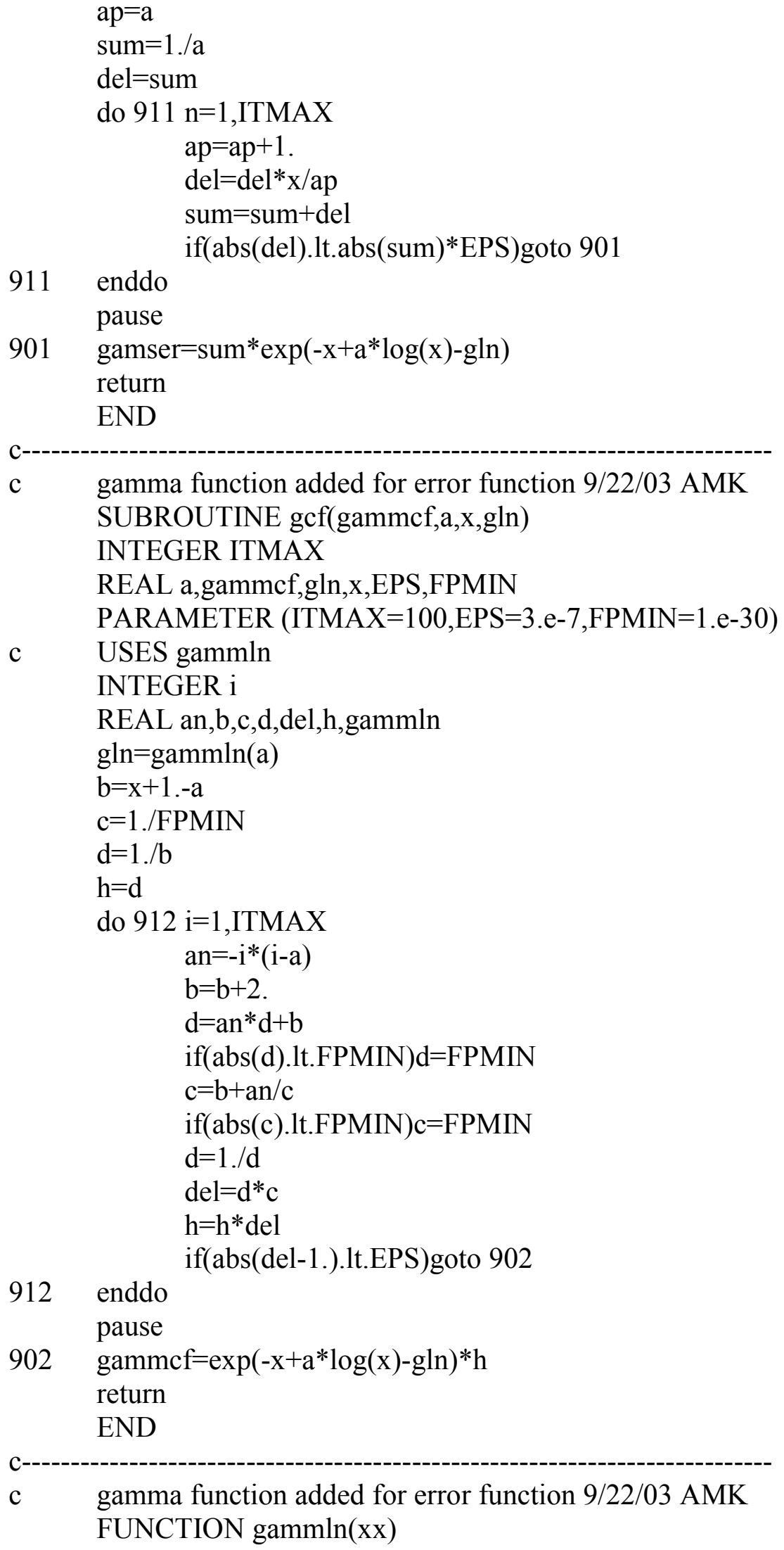


REAL gammln,xx

INTEGER $\mathrm{j}$

DOUBLE PRECISION ser,stp,tmp,x,y,cof(6)

SAVE cof,stp

DATA cof,stp/76.18009172947146D0,-86.50532032941677D0, 24.01409824083091D0,-1.231739572450155D0,.1208650973866179D-2, . $-.5395239384953 \mathrm{D}-5,2.5066282746310005 \mathrm{D} 0 /$

$$
\mathrm{x}=\mathrm{xx}
$$

$\mathrm{y}=\mathrm{x}$

$\operatorname{tmp}=\mathrm{x}+5.5 \mathrm{~d} 0$

$\operatorname{tmp}=(\mathrm{x}+0.5 \mathrm{~d} 0) * \log (\mathrm{tmp})-\mathrm{tmp}$

ser $=1.000000000190015 \mathrm{~d} 0$

do $913 \mathrm{j}=1,6$

$\mathrm{y}=\mathrm{y}+1 . \mathrm{d} 0$

$\operatorname{ser}=\operatorname{ser}+\operatorname{cof}(j) / y$

913 enddo

gammln $=$ tmp $+\log \left(\operatorname{stp}^{*} \operatorname{ser} / \mathrm{x}\right)$

return

END 
West Virginia University

Department of Physics

PO Box 6315

Morgantown, WV 26506

(304) 293-3422 ext. 1414

\section{Amy M. Keesee}

ams_510@yahoo.com

308 1/2 Magnolia Ave.

Clarksburg, WV 26301

(304) 282-3527

\section{EDUCATION}

West Virginia University, Morgantown, WV. Doctor of Philosophy in Plasma Physics, 2006. Master of Science in Physics, 2003. GPA 3.73.

Davidson College, Davidson, NC. Bachelor of Science in Mathematics, 2000. Graduated magna cum laude with honors in Applied Mathematics. GPA 3.82.

\section{HONORS}

Department of Energy Fusion Energy Sciences Fellow, 2001-04.

Sigma Pi Sigma, National Physics Honor Society, inducted 2001.

Teaching Assistant of the Year, 2000-01.

Phi Beta Kappa, inducted 2000.

Omicron Delta Kappa, National Leadership Honor Society, inducted 2000.

Bernard Society, Davidson College Mathematics Honor Society, inducted 1998. President, 1999-2000.

C. F. Meyers Jr. - Burlington Honor, 1999.

Davidson College Friends of the Arts, 1997-99.

\section{RESEARCH EXPERIENCE}

Graduate Research Assistant, 2001-2006. Studied radial profile of argon neutrals in a helicon source plasma using diode laser-based laser-induced fluorescence, passive emission spectroscopy, and atomic physics modeling with Matlab and Fortran. Designed and built system to heat iodine cell for laser wavelength reference. Performed laser-induced fluorescence measurements of argon neutrals using new scheme with diode laser. Experience with diode laser- and dye laser-based laser-induced fluorescence, building and taking data with Langmuir probes, writing LabWindows code for data acquisition. Studied remote ion temperature measurements of terrestrial magnetosphere using IDL to process energetic neutral atom imaging data from the IMAGE satellite.

East Asia and Pacific Summer Institute, 2004. Eight-week research program at Australian National University, sponsored by the National Science Foundation. Set up diode laser-based laser-induced fluorescence diagnostic system on helicon source at ANU. Measured parallel flow speeds of argon ions accelerated by a double layer potential drop.

Department of Energy Fusion Energy Sciences Practicum, 2002. Spent summer at Princeton Plasma Physics Laboratory working on the National Spherical Torus Experiment. Studied edge turbulence using IDL to track movement of turbulent filaments of neutral helium measured with the Gas Puff Imaging diagnostic.

Center for Nonlinear Analysis Summer Institute, 1999. Summer research program in mathematics at Carnegie-Mellon University. Studied chaotic behavior of Newton's method for cubic polynomials in the complex plane using TruBasic. 
Research Experience for Undergraduates, 1998. Summer research program in solar physics at National Solar Observatory in Tucson, AZ. Studied solar irradiance using $\mathrm{C}$ and IDL to process EUV data from the SOHO satellite.

\section{TEACHING AND OUTREACH EXPERIENCE}

Take our Daughters and Sons to Work Day, 2005. Participated in "Adventures in Physics" workshop for grade-school-aged children of University faculty.

Association for Women in Science, 2004-2006. Founder and President of WVU student chapter. Organized panel of women scientists, set up mentoring program with state chapter of AWIS, created website for organization including list of funding and information resources for women in science.

Expanding Your Horizons, 2004-2006. Presented workshops on physics topics for middle school-age girls. Assisted with recruiting and organization of workshop presenters.

FIRST, 2003-2005. Assistant coach for middle school Lego Robotics team. Helped students with robot design, building, and programming using RoboLab (based on LabView) software. Website judge for Pittsburgh regional high school competition.

Literacy Volunteers of West Virginia, 2001-2005. Tutor English as a Second Language and Adult Basic Reading.

Graduate Teaching Assistant, 2000-01. Taught Introductory Physics Laboratories with Pasco Interface.

Tutor, 1998-present. Tutored middle school through undergraduate math and physics in group and individual settings.

\section{PUBLICATIONS}

A.M. Keesee and E.E. Scime, 'Neutral argon density profile determination by comparison of spectroscopic measurements and a collisional-radiative model,' in publication at Rev. Sci. Instrum. (2006)

A.M. Keesee, E.E. Scime, C. Charles, A. Meige, and R.W. Boswell, 'The ion velocity distribution function in a current-free double layer,' Phys. Plasmas (2005).

X. Sun, A.M. Keesee, C. Biloiu, E.E. Scime, A. Meige, C. Charles, and R.W. Boswell, 'Observations of ion-beam formation in a current-free double layer,' Phys. Rev. Lett. (2005).

A.M. Keesee, E.E. Scime, and R.F. Boivin, 'Laser-induced fluorescence measurements of three plasma species with a tunable diode laser,' Rev. Sci. Instrum. (2004).

S.J. Zweben, R. Maqueda, D. Stotler, A. Keesee, C. Bush, S. Kaye, B. LeBlanc, J. Lowrance, V. Mastracola, R. Maingi, G. Renda, 'High speed imaging of edge turbulence in NSTX,' Nuc. Fusion (2004).

J.L. Kline, M.M. Balkey, P.A. Keiter, E.E. Scime, A.M. Keesee, X. Sun, R. Hardin, C. Compton, R.F. Boivin, M.W. Zintl, 'Ion dynamics in helicon sources,' Phys. Plasmas (2003).

J.L. Kline, E.E. Scime, R.F. Boivin, A.M. Keesee, X. Sun, 'Slow wave ion heating in the HELIX helicon source,' Plasma Sources Sci. Technol. (2002).

E.E. Scime, A.M. Keesee, R.S. Spangler, M.E. Koepke, C. Teodorescu, E.W. Reynolds, 'Evidence for thermal anisotropy effects on shear modified ion acoustic instabilities,' Phys. Plasmas (2002).

E. Scime, A.M. Keesee, J.-M. Jahn, J.L. Kline, C.J. Pollock, M. Thomsen, 'Remote ion temperature measurements of Earth's magnetosphere: Medium energy neutral atom (MENA) images,' Geophys. Res. Lett. (2002).

J. Kline, E. Scime, R. Boivin, A.M. Keesee, X. Sun, and V. Mikhailenko, 'RF absorption and ion heating in helicon sources,' Phys. Rev. Lett. (2002). 
A. Smith, 'Basins of roots and periodicity in Newton's Method for cubic polynomials,' RoseHulman Undergrad. Math Journal (2000). (published under maiden name)

\section{INVITED TALKS}

A.M. Keesee, E.E. Scime, and A. Bogaerts, 'Neutral density profile determination by comparison of spectroscopic measurements and a collisional-radiative model,' High Temperature Plasma Diagnostics, May 2006.

A.M. Keesee, 'Ions and neutral atoms in a prototype high density plasma thruster,' Institute for Scientific Research, 15 Sept. 2005.

A.M. Keesee, 'Easy Rocket Engines: Just Add lons!,' Davidson College, 14 Feb. 2005.

A.M. Keesee, E.E. Scime, C. Charles, and R. Boswell, 'Laser-Induced Fluorescence Measurements at West Virginia University and Australian National University,' Australian National University, 17 Aug. 2004.

\section{CONFERENCE PRESENTATIONS}

A.M. Keesee, E.E. Scime, and A. Bogaerts, 'Neutral density profiles in a helicon source,' APSDivision of Plasma Physics, Oct. 2005.

A.M. Keesee, X. Sun, C. Biloiu, E.E. Scime, A. Meige, C. Charles, and R. Boswell, 'Laserinduced fluorescence measurements of ions in laboratory double layers,' Interrelation Between Plasma Experiments in Laboratory and Space, July 2005.

A.M. Keesee, E.E. Scime, C. Charles, and R. Boswell, 'Laser-Induced Fluorescence Measurements of lons Accelerated by a Double Layer,' APS-Division of Plasma Physics, Nov. 2004.

A.M. Keesee and E.E. Scime, 'Radial profiles of neutral argon in a helicon source,' International Conference on Plasma Science, June 2004.

A.M. Keesee, E.E. Scime, and R.F. Boivin, 'LIF measurements of three plasma species with a tunable diode laser,' APS-Division of Plasma Physics, Oct. 2003 and High Temperature Plasma Diagnostics, Apr. 2004.

A.M. Keesee, E.E. Scime, C. Biloiu, C.S. Compton, R.A. Hardin, and X. Sun, 'Minor ion heating in magnetospherically relevant plasmas,' Interrelation Between Plasma Experiments in Laboratory and Space, June 2003.

A.M. Keesee, R. Maqueda, W.M. Nevins, and S.J. Zweben, 'Structure and motion of edge turbulence on NSTX,' APS-Division of Plasma Physics, Nov. 2002.

A.M. Keesee, E.E. Scime, and C.J. Pollock, 'Ion heating in the Earth's magnetosphere during substorm and storm-time,' APS-Division of Plasma Physics, Oct. 2001 and AGU, Dec. 2001.

A. Smith and R. Neidinger, 'Basins of roots and periodicity in Newton's Method for cubic polynomials,' Mathematical Association of America, 2000.

\section{REFERENCES}

Earl Scime, Department of Physics, West Virginia University, PO Box 6315, Morgantown, WV 26506. (304) 293-3422 ext. 1437. escime@wvu.edu

Christine Charles, Research School of Physical Sciences and Engineering, Australian National University, Oliphant Building 60, Mills Road, Canberra ACT 0200, Australia. +6126125 8068. christine.charles@anu.edu.au

Mark Koepke, Department of Physics, West Virginia University, PO Box 6315, Morgantown, WV 26506. (304) 293-3422 ext. 1456. mkoepke@wvu.edu 